\author{
Stephen W. Kercel \\ Oak Ridge National Laboratory, P.O. Box 2008, Oak Ridge, TN 37831 \\ Marvin B. Klein and Bruno Pouet \\ Lasson Technologies, 6059 Bristol Parkway, Culver City, CA 90230
}

November 2000

\author{
Prepared by \\ OAK RIDGE NATIONAL LABORATORY \\ Oak Ridge, Tennessee 37831-6004 \\ Managed by \\ UT-Battelle, LLC \\ for the \\ U.S. DEPARTMENT OF ENERGY \\ Under contract DE-AC05-00OR22725
}


This page intentionally left blank. 


\section{CONTENTS}

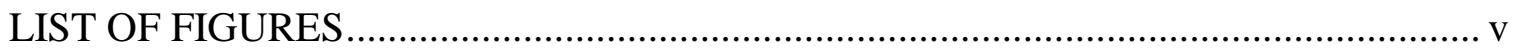

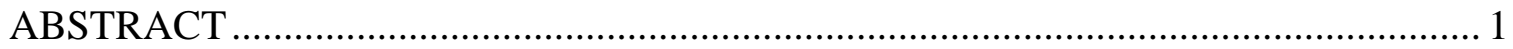

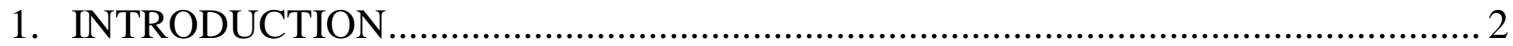

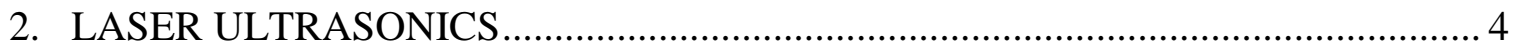

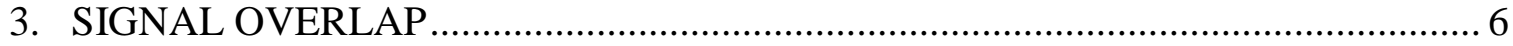

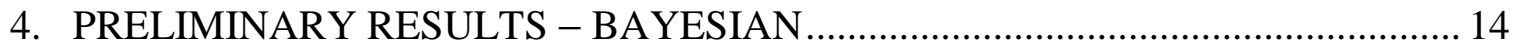

5. PRELIMINARY RESULTS - DISCRETE WAVELETS …................................. 19

6. THE OBSERVED EFFECT OF WELD FLAWS ON LAMB WAVE MODES ....... 22

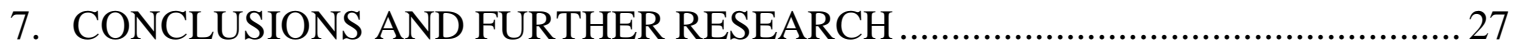

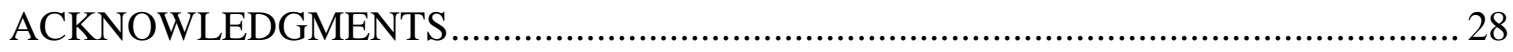

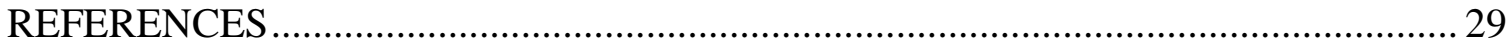

APPENDIX A - RESULTS OF BAYESIAN ANALYSIS....................................... 31 
This page intentionally left blank. 


\section{LIST OF FIGURES}

Figure 1. Schematic Drawing of Normal Laser Butt Weld and Defective Welds........... 3

Figure 2. Weld-Joint Sensor as Transducer In Closed-Loop Control Scheme................. 3

Figure 3. Schematic Diagram of Weld Inspection Geometry Using Transmitted Lamb

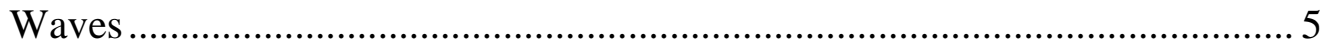

Figure 4. Signal Overlapped in Time and Frequency.......................................... 6

Figure 5. Likelihood of Fit of Signal to Gabor Function......................................... 7

Figure 6. Most Likely Signal and Residual ........................................................... 8

Figure 7 Other Components of Overlapped Signal .............................................. 8

Figure 8 Wavelet and Scaling Functions for 10-Coefficient Least Asymmetric

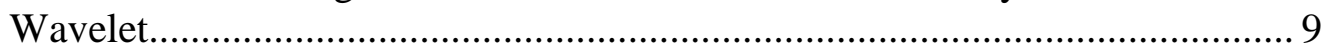

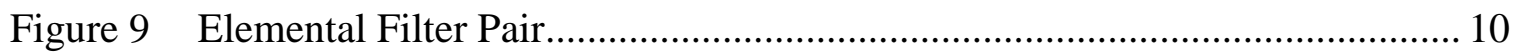

Figure 10 Implementation of the Discrete Wavelet Transform................................... 11

Figure 11 Implementation of the Wavelet Packet Transform ................................... 12

Figure 12 Cumulative Energy of the Same Signal In Different Transform Spaces ....... 13

Figure 13 Successive Scans Across a Flawed Weld.................................................. 15

Figure 14 Likeliest Component of Each Scan ........................................................ 16

Figure 15 Second Likeliest Component of Each Scan ......................................... 16

Figure 16 Third Likeliest Component of Each Scan .............................................. 17

Figure 17 Separation of Signal Energies by Wavelets ......................................... 20

Figure 18 Denoising a Laser Ultrasonic Signal ................................................... 21

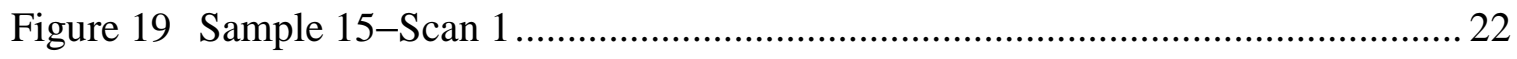

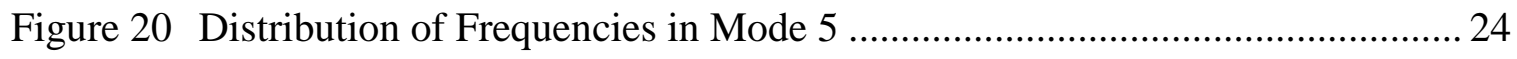

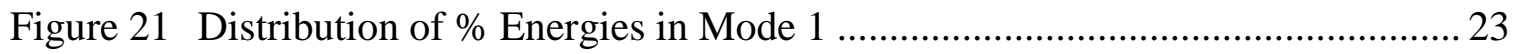




\section{LIST OF FIGURES (cont'd)}

Figure 22 Weld Features in 2D Feature Space .................................................... 24

Figure 23 Quartile Ellipses for the Two Classes in Feature Space.............................. 24 


\title{
IN-PROCESS DETECTION OF WELD DEFECTS USING LASER-BASED ULTRASONIC LAMB WAVES
}

\begin{abstract}
Laser-based ultrasonic (LBU) measurement shows great promise for on-line monitoring of weld quality in tailor-welded blanks. Tailor-welded blanks are steel blanks made from plates of differing thickness and/or properties butt-welded together; they are used in automobile manufacturing to produce body, frame, and closure panels. LBU uses a pulsed laser to generate the ultrasound and a continuous wave $(\mathrm{CW})$ laser interferometer to detect the ultrasound at the point of interrogation to perform ultrasonic inspection. LBU enables in-process measurements since there is no sensor contact or near-contact with the workpiece.
\end{abstract}

The authors have used laser-generated plate (Lamb) waves to propagate from one plate into the weld nugget as a means of detecting defects. This report recounts an investigation of a number of inspection architectures based on processing of signals from selected plate waves, which are either reflected from or transmitted through the weld zone. Bayesian parameter estimation and wavelet analysis (both continuous and discrete) have shown that the LBU time-series signal is readily separable into components that provide distinguishing features, which describe weld quality. The authors anticipate that, in an on-line industrial application, these measurements can be implemented just downstream from the weld cell. Then the weld quality data can be fed back to control critical weld parameters or alert the operator of a problem requiring maintenance. Internal weld defects and deviations from the desired surface profile can then be corrected before defective parts are produced.

The major conclusions of this study are as follows. Bayesian parameter estimation is able to separate entangled Lamb wave modes. Pattern recognition algorithms applied to Lamb mode features have produced robust features for distinguishing between several types of weld defects. In other words, the information is present in the output of the laser ultrasonic hardware, and it is feasible to extract it. Wavelet analysis produces results that are almost as good as Bayesian, but execute a thousand times faster. This study demonstrates the principle that it is feasible to construct a laser ultrasonic system to detect weld defects in thin metal parts on-line in real-time, and to classify the defects according to type.

Keywords: "laser-based ultrasonic," "weld inspection," "on-line inspection," "Bayesian," and "wavelet." 
This page intentionally left blank. 


\section{INTRODUCTION}

The automotive industry is continually re-engineering its manufacturing processes to effect cost savings, enhance quality, reduce weight, and improve safety. One new process with all these attributes is associated with the fabrication of sheet metal panels for auto body manufacture. Different areas of the body have varying requirements for strength and corrosion resistance. Older manufacturing techniques either used single panels of sheet metal with stiffeners and protective coatings, or multiple panels with the proper characteristics which were attached separately to the body frame. In a new design-for-manufacturing approach, manufacturers are now producing large sheet metal panels or blanks made from smaller, individually engineered panels that are butt-welded together using a $\mathrm{CO}_{2}$ or Nd:YAG laser welding process. With this approach, panels with differing thickness, metallurgy, or surface treatment can be joined to provide the desired attribute only in positions where it is required. Compared to conventional methods, the advantages of these tailor-welded sheet metal blanks are:

- less tooling and better integration of parts,

- forming with a single set of dies,

- reduction in manufacturing steps and in part count,

- superior dimensional control,

- reduction in overall weight,

- improved crash energy management,

- corrosion resistance, higher strength only where required,

- lower net cost, and

- better fit during assembly, resulting in less body noise.

In the United States, the production of tailor-welded blanks for 1997 is estimated to be 8 million blanks worth approximately $\$ 25$ million and made from 110,000 tons of sheet steel. The corresponding measure of welds produced is on the order of 10 miles per day. This production rate is expected to increase by a factor of 10 by the turn of the century.

In the blank welding process, the adjacent edges of the two panels to be welded are held at a fixed gap or in contact and the panels are then advanced under the stationery laser fixture. The most critical weld condition that must be controlled is the integrity and shape of the weld nugget formed between adjacent base metal surfaces. The desired transition from one surface to the other is smooth and flat, as shown in Figure 1. Either a concave or a convex surface is a sign of a lower strength weld. Typically, a concave surface can arise from poor fit-up or seam tracking, incomplete weld penetration or excessive weld penetration. Additionally, any crack or lack of fusion is a weld defect. 


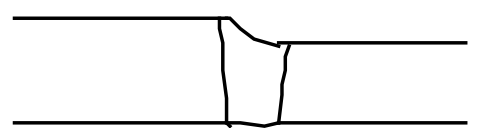

Normal butt weld

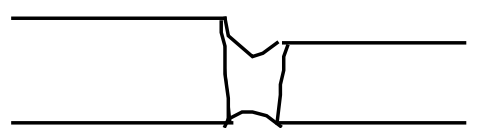

Poor interface

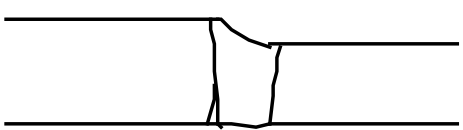

Crack, lack of fusion

Figure 1. Schematic Drawing of Normal Laser Butt Weld and Defective Welds.

On-line monitoring of weld quality is a crucial unsolved problem in the manufacture of tailorwelded blanks. The goal is to be able to detect the full range of defects that may be present. At the current time, process monitors (e.g., weld speed, laser current) have been implemented, and control of these parameters to maintain the desired weld quality has been attempted. However, these open-loop controls are not sufficiently effective. A closed loop control scheme is required, as shown in Figure 2. However, conventional sensing technology does not provide a suitable transducer.

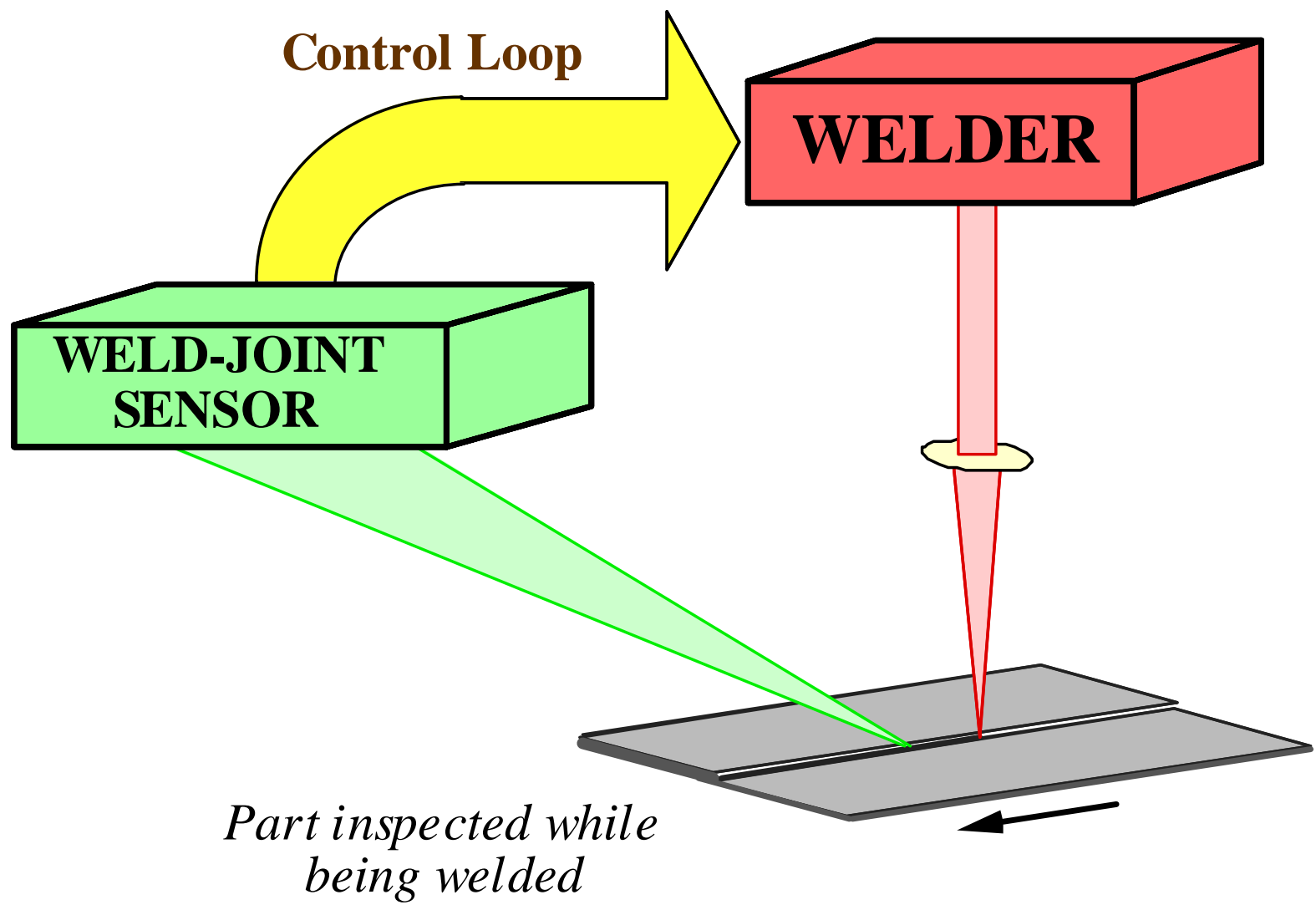

Figure 2. Weld-Joint Sensor As Transducer In Closed-Loop Control Scheme. 
Several acoustic, optical, and ultrasonic sensors have been studied. Acoustic emission from the weld process (measured with an external microphone) was found to be noisy and not a full measure of weld quality. Optical sensors based on measurement of the spectral emission from the weld plasma or fusion zone have been investigated. Some correlation with weld quality has been observed, but the discrimination is not strong. Finally, ultrasonic inspection using electromechanical acoustic transducers (EMAT) has been studied, and has been shown to be unable to detect all the flaws.

It is clear that the need remains to implement a sensor that could directly measure the nugget integrity and surface profile, and thus determine the strength of the weld. One approach is to measure the deflection angle of a laser beam reflected from the nugget surface. Such an approach has been attempted, but poor surface quality has prevented reliable measurement of the surface profile. In addition, both sides of the blank would have to be interrogated. In the absence of any reliable automated sensor, welded blanks in the factory are now inspected by visual means on a statistical basis. Destructive sectioning with micrographic examination is sometimes used to augment the visual inspection. If defective welds are found then the output from the most or the entire shift must be inspected and the welder must be recalibrated. Clearly, this approach leads to added cost in energy, materials, and labor. As an alternative to this reactive welder maintenance approach, it would be desirable to develop a sensor that would monitor the desired weld property directly and allow proactive intervention to correct the weld process in real time or to alert the operator that maintenance is required.

\section{LASER ULTRASONICS}

A technology that shows great promise for on-line measurement of weld quality in tailor-welded blanks is laser-based ultrasound (LBU). LBU is a technique for performing ultrasonic inspection using a pulsed laser to generate the ultrasound and a separate CW laser interferometer to detect the ultrasound at the point of interrogation. [1] Laser-based ultrasound has several significant advantages for this application. No sensor contact (or near-contact) with the workpiece is required, thereby allowing in-process measurements in the harsh welder environment (i.e., high temperatures, turbulent atmospheric environment, vibrating parts with rapid lateral motion). Both free-space and fiber-based delivery of optical energy can be implemented.

The authors are investigating the use of laser-generated plate (Lamb) waves propagating from one plate into the weld nugget as a means of determining the surface profile. The investigation is considering a number of inspection architectures based on processing of signals from selected plate waves, which are either reflected from, or transmitted through the weld zone. One goal is to identify the simplest generation technique, wave mode, wave propagation geometry, feature extraction process, and pattern recognition algorithm that will provide effective identification of defective welds. It is anticipated that these measurements can be implemented just downstream from the weld cell (see Figure 3), so that weld quality data can be fed back to control critical weld parameters (or alert the operator of a problem requiring maintenance). Any deviation or defects from the desired surface profile can then be corrected before defective parts are produced. 


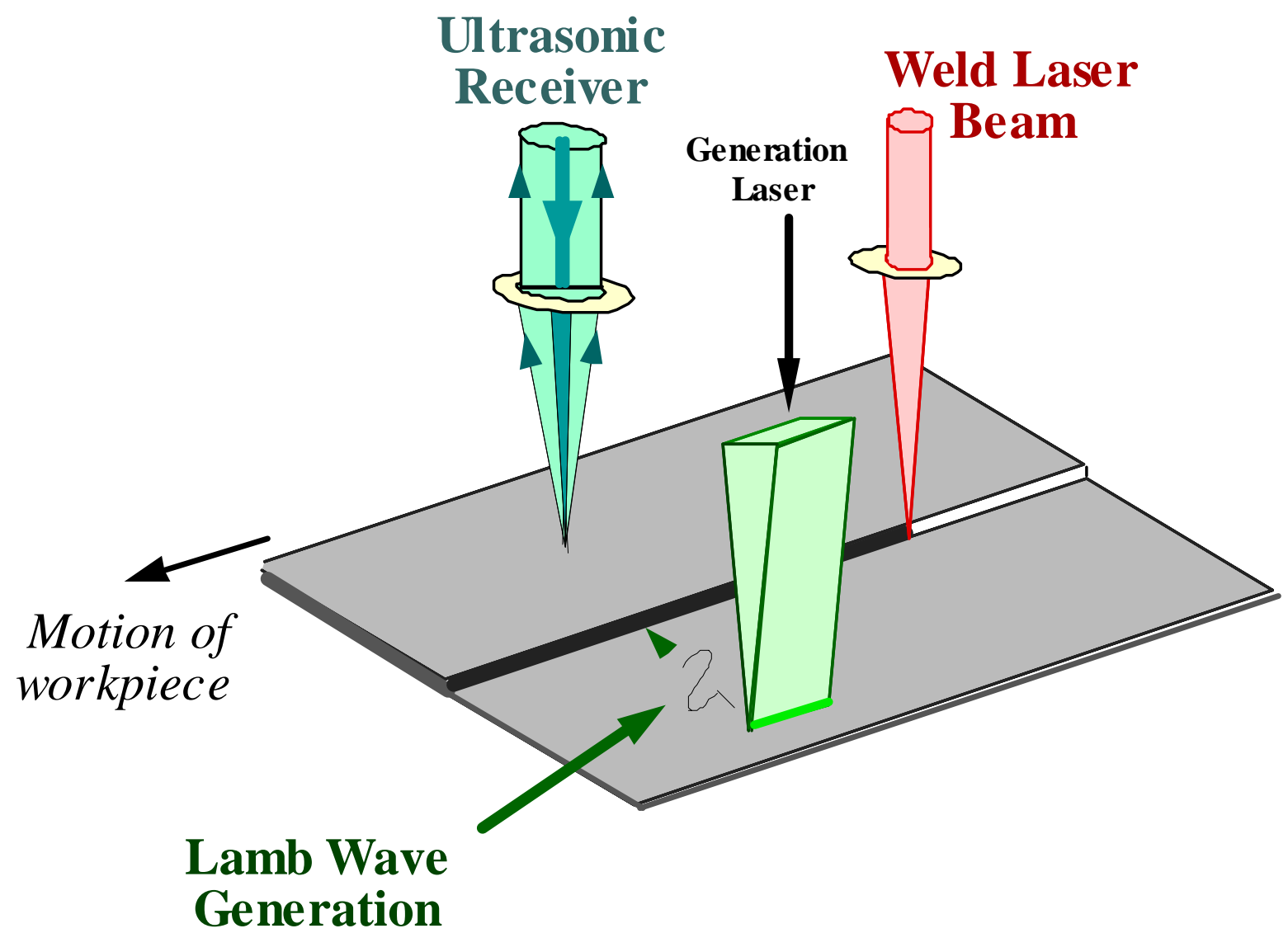

Figure 3. Schematic Diagram of Weld Inspection Geometry Using Transmitted Lamb Waves.

Recent advances in optical receiver technology can overcome some of the disadvantages of conventional laser-ultrasonic methods. [1] Adaptive interferometric receivers under development at Lasson allow the processing of speckled signal beams that result from the interrogation of machined surfaces. In conventional interferometric receivers, speckle is a source of phase error that can greatly reduce the receiver signal-to-noise ratio. In conventional LBU technology, multiple speckles can be avoided by focusing tightly to a single speckle, but this approach has the risk of signal "dropouts" due to the presence of "dark speckles." The other major advantage of the new adaptive receivers is that they are insensitive to noise at lower frequencies caused by workpiece vibration and by welder-induced turbulence in the path of the receiver probe laser.

One challenge in implementing LBU for process control is the relatively low signal-to-noise ratio. This problem could be overcome by using more sophisticated signal processing for LBU than has been used in the past. [2] The signals produced by the LBU receiver are non-stationary transients in the time-domain, and real-time wavelet analysis should lead to the most efficient method of feature extraction. Furthermore, embedded wavelet processes are implementible in real-time hardware, and are effective for many pattern recognition problems for transient signals in severe noise. [3,4,5] 


\section{SIGNAL OVERLAP}

One of the major problems in the analysis of signals from ultrasonic sensors is the fact that the signal of interest is often overlapped with many other signals [6]. The feature of interest (such as a localized weld defect) produces a signal that contains desired information (such as the distance of the weld defect from the point of ultrasonic excitation). However, other features (such as the edges of the workpiece) also produce signals that overlap the signal of interest, but contain no relevant information. In addition, the signals are inherently non-stationary, and equally inseparable in the time domain and the Fourier frequency domain. Finally, the signal of interest may be considerably weaker than the obscuring signals.

The key to the signal-processing problem is to separate the desired signal from the undesired signals while not destroying the desired information with the process. To appreciate the idea, consider a sensor signal that is the sum of four overlapping Gabor functions plus a low-level Gaussian-distributed random function. Also consider that the information of interest is contained in one of the Gabor functions. (Note: A Gabor function is a Gaussian-windowed sinusoid. Its waveform is completely characterized by four parameters, the frequency and phase of the sinusoid, and the peak location and width of the window.)

In this example, suppose that each of the Gabor functions is produced by a different physical feature, and that we can tell something about the feature (for example, its location in space) by examining the parameters of the Gabor function associated with that specific feature. The problem is that we must disentangle the underlying Gabor functions given the overlapped signal. As shown in Figure 4, the signal is the sum of a low level of Gaussian noise and four Gabor functions, with normalized frequencies of $0.025,0.05,0.025$, and 0.05 , and window peaks at time delays of 600, 1400, 1400, and 600 respectively. The components are not conveniently separable in either the time or frequency domains.
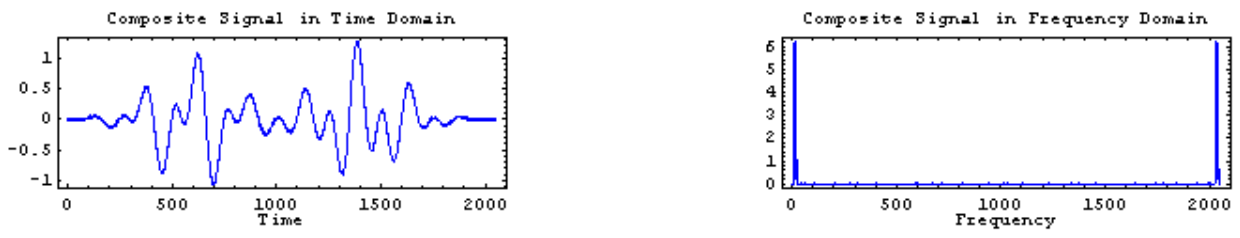

Figure 4. Signal Overlapped in Time and Frequency.

Suppose we have prior knowledge from our understanding of the underlying physical process that the signal should contain one or more Gabor functions, but we have no prior knowledge of the parameters such as frequency or the time of the window peak. By the methods of Bayesian parameter estimation [7], we can use the Gabor function as a model, and guess a frequency and a time. We can project the signal shown in Figure 4 onto the model, and compute the log likelihood that the Gabor function with the guessed parameters fits the data. If we repeatedly guess sets of parameter values, and plot the resulting log likelihoods against the guessed parameter values, we obtain the plot shown in Figure 5. 
Another way of saying this is that Figure 5 is the projection into log likelihood space of the signal shown in Figure 4. When we examine this composite signal in log likelihood space, we see four well-separated components, and expect that we should be able to recover each component, one by one. The two parameters are, $\omega$, the oscillation frequency $(0-0.06)$ and $\tau$, the time of occurrence (0-2000) of the event. The vertical dimension is the log-likelihood that the observed data contains a Gabor function with the given pair of the parameter values.

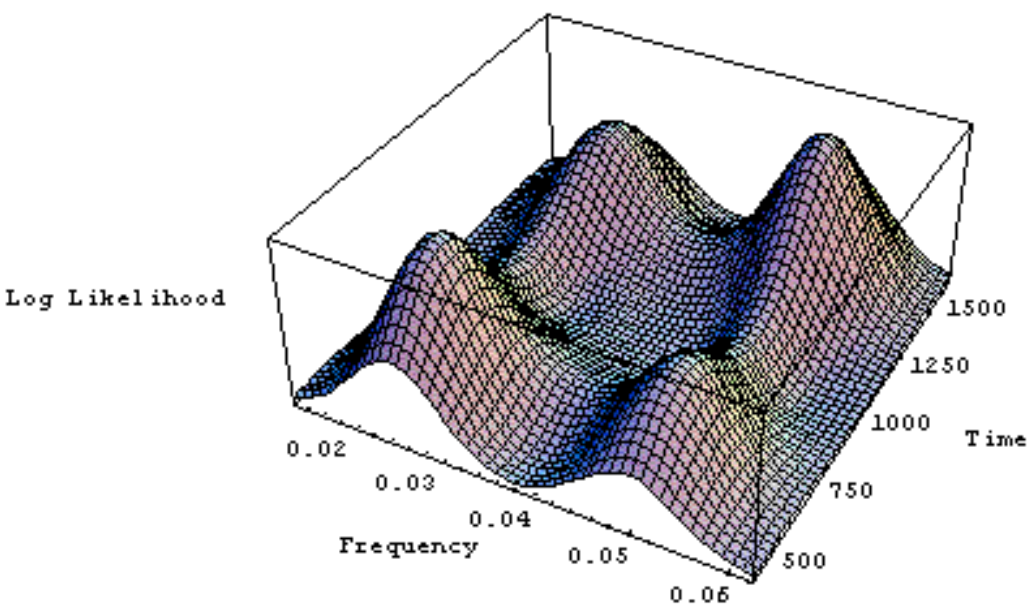

Figure 5. Likelihood of Fit of Signal to Gabor Function.

Given the signal shown in Figure 4, the most likely Gabor function that fits the signal, is the one whose parameters lead to the greatest log likelihood. Using a global optimizing algorithm, with the objective function being the log likelihood as a function of the time and frequency parameters, we can readily find the optimal combination of parameters. (Note that local optimization algorithms such as gradient descent are vulnerable to being trapped in local optima.) As indicated by the peak in Figure 4, the optimum value of the objective function occurs at a time of 605.4 and a frequency of 0.0248 .

The most likely Gabor function in the signal shown in Figure 4 is plotted in the left-hand plot in Figure 6. This is the Gabor function whose parameters are found at the global optimum in Figure 5. When this estimated signal is subtracted from the signal in Figure 4, the residual in the righthand plot of Figure 6 is obtained. Note that the most likely Gabor function has been separated from the signal without disturbing the other information in the signal. 

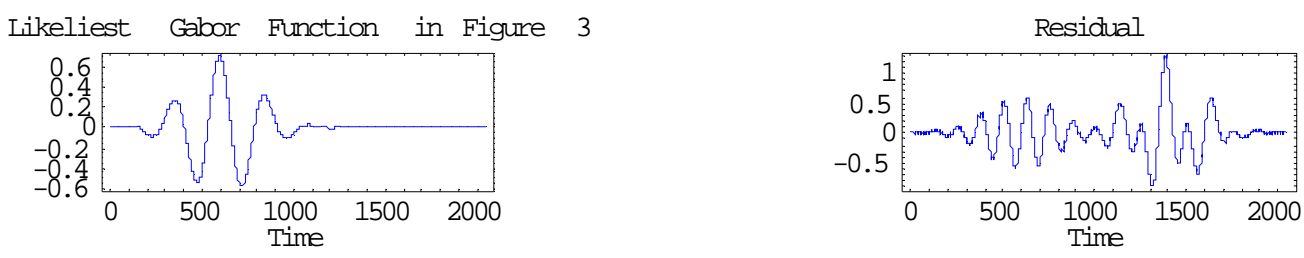

Figure 6. Most Likely Signal and Residual.

Since we have reason to believe that the signal should contain several Gabor functions plus noise, we can apply precisely the same methods to the residual, and obtain the next most likely signal, and its residual with the next likeliest Gabor function removed. In Figure 7 we see the results of repeating this process until the residual is reduced to noise. The second through fourth likeliest Gabor functions have times of 1397.9, 612.2 and 1400.9 and frequencies of 0.0251, 0.050 , and 0.050 respectively. The residual after removing the four likeliest Gabor functions is noise and cumulative rounding error.

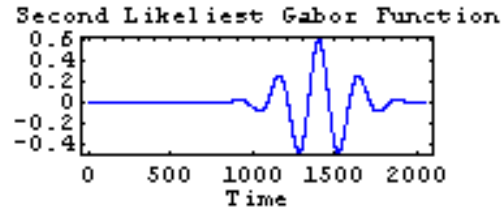

Fourth Likel iest Gabor Function

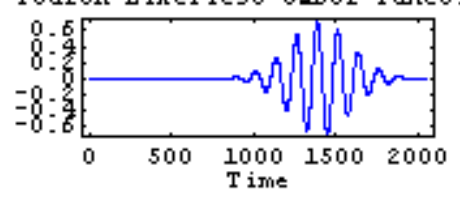

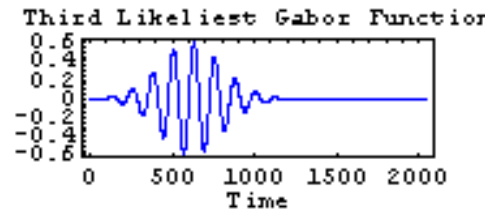

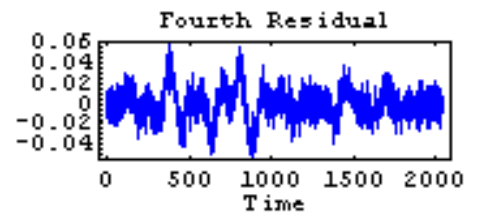

Figure 7. Other Components of Overlapped Signal.

The point of the foregoing discussion is to demonstrate that overlapping signals can be separated without disturbing each other. Bayesian parameter estimation provides the optimal estimates of the models underlying the signal, however it is computationally costly. For the purposes of online real-time monitoring, the goal is to find a method that is almost as good as Bayesian, but much faster.

In laser ultrasonic, the signals of interest are oscillating bursts. This suggests that wavelet analysis might produce acceptable performance for an on-line instrument. The wavelet basis function is an oscillating burst, and the discrete wavelet transform is implemented as a bank of computationally inexpensive finite impulse response (FIR) digital filters.

The idea behind wavelet analysis is that the signal can be considered as the weighted sum of overlapping wavelet functions [8]. In fact, any signal of finite bandwidth and finite duration can be completely characterized as a weighted sum of a finite number of scaled and shifted versions of the underlying wavelet. The concept is similar to Fourier analysis, in which the time series signal can be considered a weighted sum of sinusoids at various frequencies, with the transform 
coefficients being the weights. The practical meaning of the wavelet transform of a signal is that each coefficient of the transform is the weight, or relative amount of information that the wavelet at that particular value of scale and shift contributes to the overall signal.

For the results reported in the next section, the wavelet analysis was performed with the Daubechies 10-coefficient least asymmetric discrete wavelet. [9] Discrete wavelets are not expressible in closed form. Plots of the wavelet and its corresponding scaling function were computed with Daubechies cascade algorithm, and are shown in Figure 8.
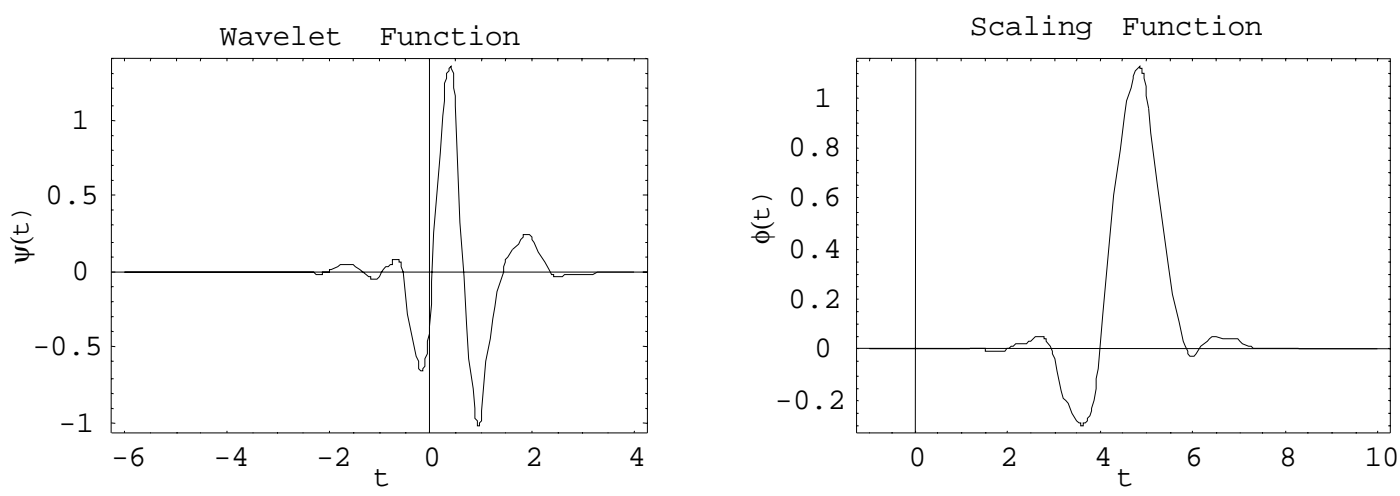

Figure 8. Wavelet and Scaling Functions for 10-Coefficient Least Asymmetric Wavelet.

Suppose that a time-domain input consists of a list of 960 evenly spaced samples of a bandlimited signal. The discrete wavelet analysis results in a list of 960 wavelet-domain coefficients output in response to each 960-element time series input signal. As sinusoids at different frequencies are orthogonal to each other, so also are scaled and translated versions of these wavelet functions orthogonal to each other. This means that Parseval's theorem holds for discrete wavelet transform; the amount of energy in the signal in the wavelet domain is exactly the same as the amount of energy in the signal in time domain. Energy is regarded as proportional to the information in the signal.

For a transient signal, such as an oscillating burst, it is expected that most of the energy in the signal will be concentrated in relatively few of the wavelet coefficients, with all the others having values very close to zero. The energy contributed by each coefficient is the square of the coefficient. The fraction of the total signal energy contributed by each coefficient is the energy of the coefficient divided by the total energy of the signal (the sum of the squares of all the samples of either the time series or all the wavelet coefficients). Sorting the wavelet coefficients from greatest to smallest, the cumulative energy of each coefficient is the fraction of total signal energy contributed by that coefficient, plus the fractions of signal energy contributed by all the larger coefficients. For a typical data set collected in this research, the largest coefficient (out of 960) contains over $30 \%$ of the total energy of the signal, and that the ten largest contain over $98 \%$ of the total signal energy. 
The discrete wavelet packet transform is a generalization of the discrete wavelet transform. As shown in Figure 9, each stage of both the wavelet transform and the wavelet packet transform consists of an elemental pair of filters (high-pass and low-pass) that splits the input signal into two decimated orthogonal components. The low-pass output is an approximation of the input signal. The high-pass output contains the details of the input signal that are missing from the approximation. There is no information in the two outputs that overlaps, and nothing is lost. The input signal can be exactly reconstructed from the two outputs.

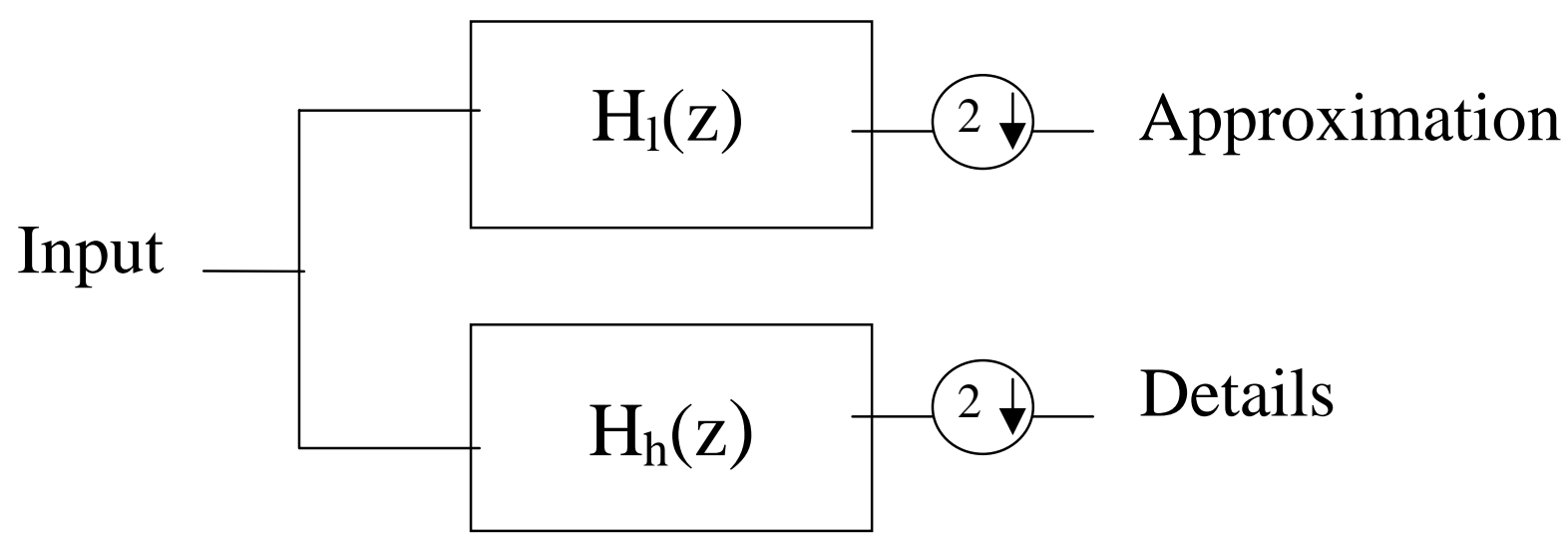

Figure 9. Elemental Filter Pair.

The discrete wavelet transform is implemented by cascading the elemental filter pairs as shown in Figure 10, and the wavelet packet is implemented by cascading them as shown in Figure 11. In the wavelet transform configuration, the low-pass output of the preceding stage is fed into an identical copy of the elemental filter pair. Thus the first approximation is further approximated, and the second set of details consists of the information present in the first approximation but absent from the second. In wavelet parlance, the output of the first high-pass filter is the set of wavelet transform coefficients of the input signal at the finest scale. The output of the next highpass filter is the set of wavelet transform coefficients of the input signal at the next finer scale. The output of the final low-pass filter is the set of scaling function coefficients. The cascade can be repeated as often as necessary, and all the outputs are orthogonal.

There is no fundamental reason why the high-pass output of the elemental filter pair cannot be split as well, and nothing to prevent repeating this process as often as necessary. A filter bank in which at least some of the high-pass outputs are split into other approximations and details implements the wavelet packet transform [10]. If the high-pass output is split whenever the lowpass output is split, then the system is a complete wavelet packet transform. However, it is not necessary for the wavelet packet to be complete. A more efficient representation of the signal may be obtained by leaving out some of the filter pairs in the cascade. Irrespective of how many filter pairs are included, all outputs are orthogonal.

For any given input signal, there is an optimal configuration of filter pairs that represents most of the input signal energy with the fewest coefficients. This is known as the best basis wavelet packet. The best basis is determined by finding the configuration of filter pairs whose output has the highest entropy [11]. 
Energy is regarded as proportional to the information in the signal. Suppose that a signal's energy consists of three major elements. In addition to the energy of the desired signal, there may be coherent or non-random signals produced as undesired, but unavoidable biasing artifacts due to the hardware. Also, there may be broadband high-frequency energy that is typically regarded as random noise. If these three energies are separable in wavelet space, then the undesired bias and noise components of the sensor output signal can be identified and subtracted from the original sensor output. What remains is the signal of interest with its features unobscured.

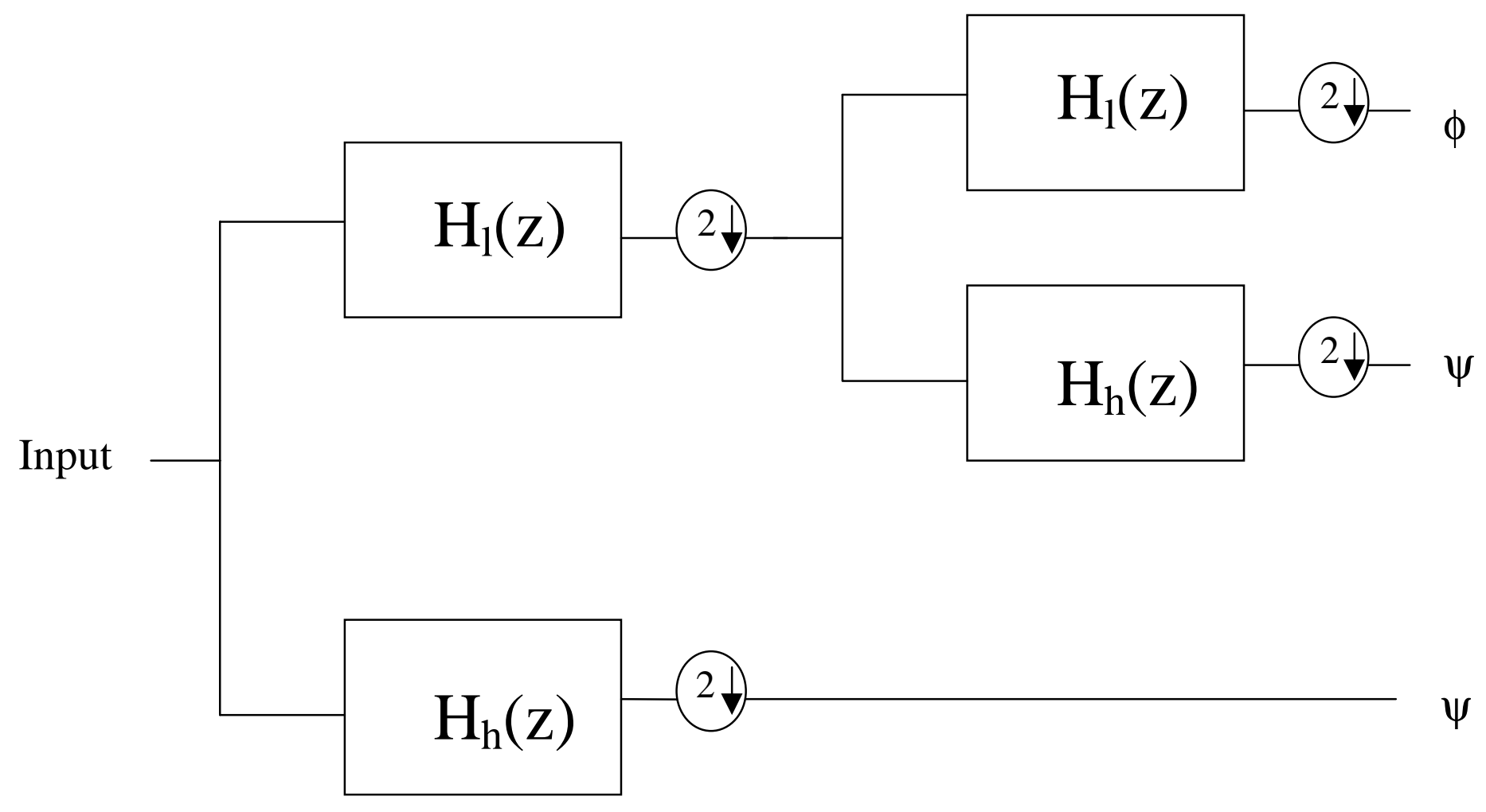

Figure 10. Implementation of The Discrete Wavelet Transform.

The reason for transforming a time-series into another domain is to try to find a more compact representation of the information in the data. The performance of the different transforms can be compared by considering how effectively each compresses the same data set. The rationale for using data compression for the first step in pattern recognition feature selection is that it is more effective to search for features in a short list of numbers than a long list. 


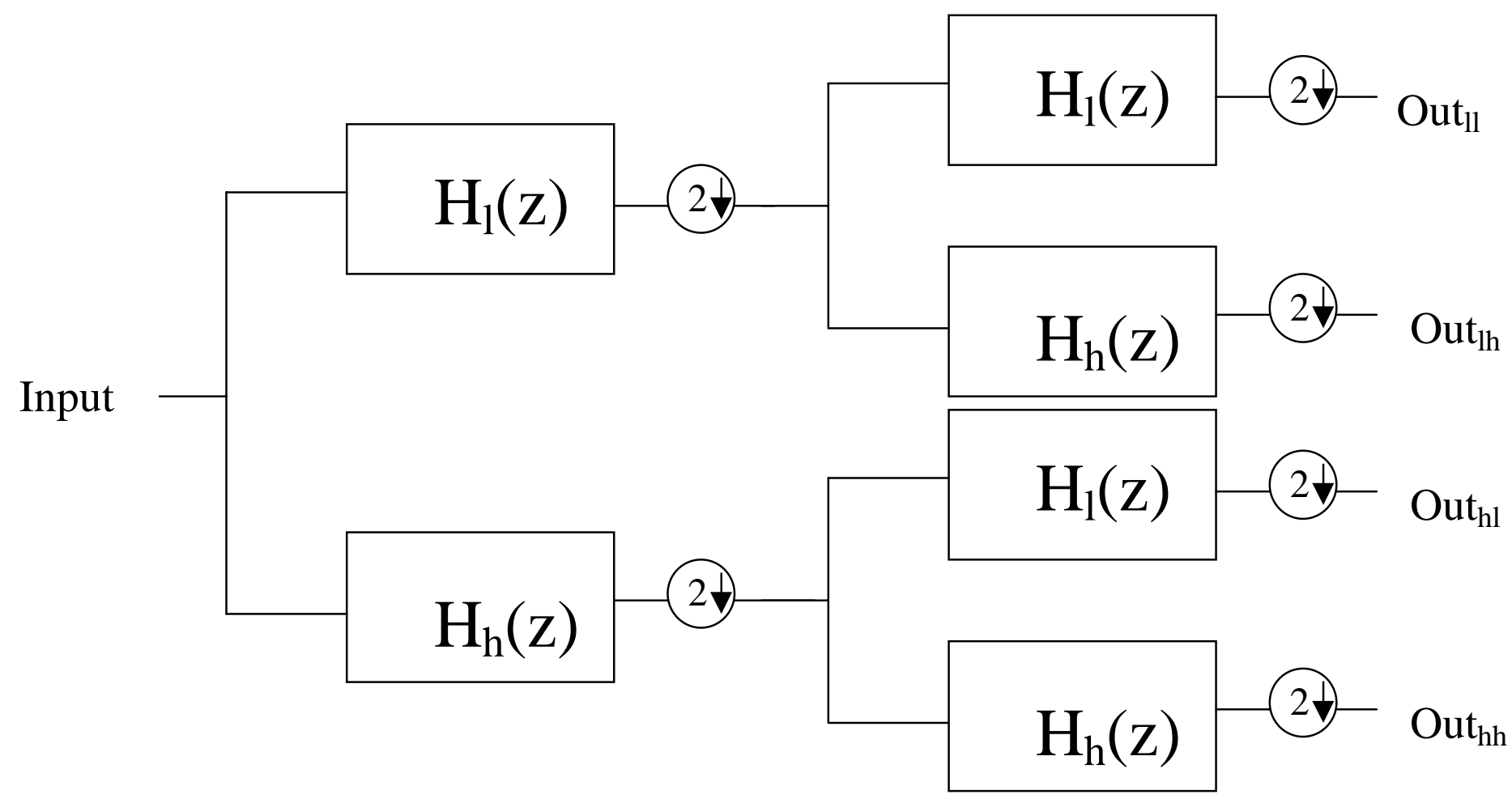

Figure 11. Implementation Of The Wavelet Packet Transform.

The effectiveness of several transforms was compared by taking the same list of 960 numbers (generated by a laser ultrasonic sensor), and producing an output list of 960 numbers. In each output list, there are relatively few big numbers, and relatively many numbers close to zero. The idea is that the useful information is in the few big numbers, and that the many small ones can be zeroed out without much loss of the underlying information. This is the conceptual basis for data compression.

The wavelet packet, the discrete wavelet, and the discrete Fourier transform are all orthogonal. Since Parseval's theorem holds, the energy in the output data set is identical to the energy in the input data set. For each transform output data set, the cumulative energy function counts up the cumulative energy in the output coefficients as energy is accumulated by counting energies, starting from the energy of the greatest transform coefficient, and moving to the smallest [12]. In the following example, we compute the normalized cumulative energy of the wavelet packet transform, the discrete wavelet transform, and the discrete Fourier transform of the same signal from a laser ultrasonic sensor.

In the upper left of Figure 12, as the plot of the first 200 members of the cumulative energy list shows, for the output of the best basis wavelet packet transform, virtually all of the information is contained in the biggest 135 members. The other 825 members are very close to zero. The upper right of Figure 5 shows the cumulative energy for the discrete wavelet transform, using the same input signal, and the same elemental filter pair (Daubechies least asymmetric 10-coefficient filter and its paraunitary companion). The lower left of Figure 12 is for the Fourier transform; it 
is clearly far less effective than the other two at compressing the signal. The lower right shows all three cumulative energies overlaid. The best basis wavelet packet transform is only marginally superior to the wavelet transform for this data set, and not worth the added cost compared to the wavelet transform.

Discrete Wavelet Transform

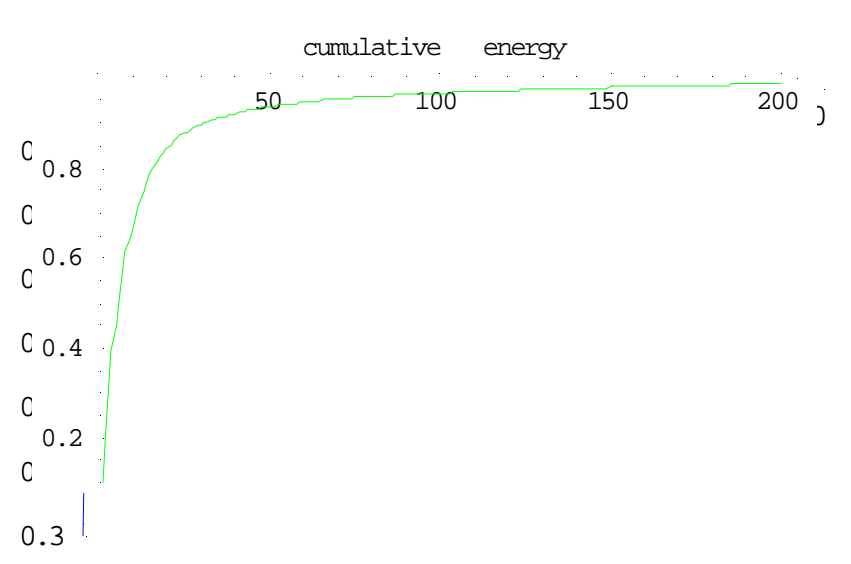

Discrete Fourier Transform

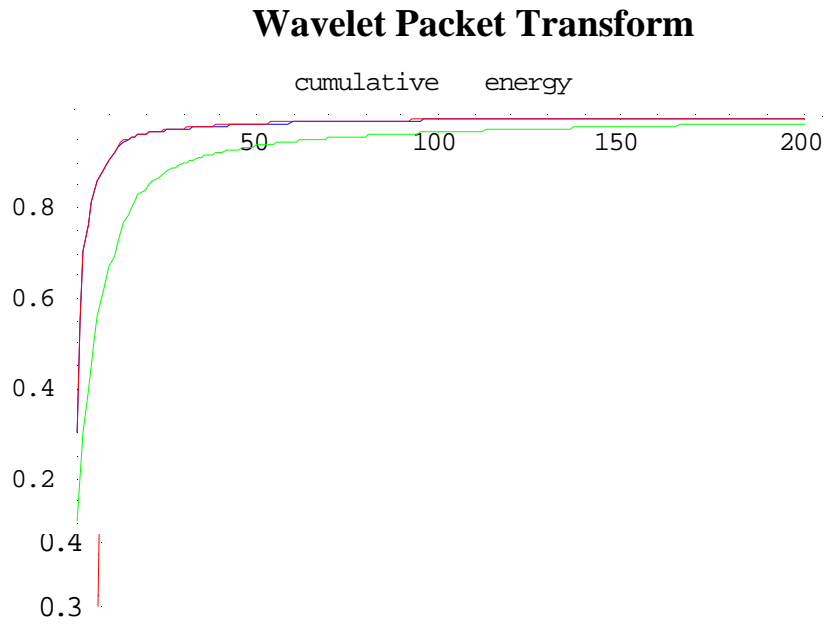

All three Overlaid

Figure 12. Cumulative Energy of The Same Signal In Different Transform Spaces.

In many cases, once the signature of interest is separated from everything else in the sensor signal, the detection of flaws in the workpiece is straightforward. Typically, the workpiece is scanned across a physical range by the LBU apparatus. Continuity of the mathematical properties of the signature across scans suggests an unflawed workpiece. The appearance of an abrupt discontinuity, such as a dramatic localized change in peak of window location (often suggesting a localized change in time of flight of an ultrasonic echo) suggests the presence of a flaw.

Consistent interpretation of weld signatures must be model-based, where the model is in some reasonable sense a description of the underlying physical reality. The various bursts that appear in the sensor output have physical causes, and in ultrasonics, the physics of the causes is typically well understood. Signal processing by the methods described in this section exploit this knowledge to wring the maximum of new and relevant information from the sensor output data, and provide an indication of confidence in the results. This is in contrast with the widely popular paradigm of signal processing in which an empirical model is surmised without respect to the underlying process. The empirical approach works for some data sets, but give no indication of when it might break down for the next unknown trial. 


\section{PRELIMINARY RESULTS - BAYESIAN}

A typical result of LBU inspection of a tailor-welded blank is shown in Figure 13. Each plot is the output voltage of a LBU photo-detector as a function of time, as the LBU system makes 30 successive scans across a workpiece with a flawed weld. It would be very difficult to detect the flaw simply from a visual inspection of these raw data.

From the data for each of the 30 scans, the most likely model (or dominant component) was computed using Bayesian parameter estimation. For each scan, the waveform of the most probable model is a chirped Gaussian-windowed sinusoid. The most probable is taken to be the dominant component of the signal. These are plotted in Figure 14. This dominant component appears to be a biasing effect characterizing the experimental setup. As shown in Table 1, this component contains approximately $95 \%$ of the signal energy for each scan.

The second likeliest component for the data of each of the 30 scans is plotted in Figure 15. For each scan, the first residual is computed by subtracting the estimated data for the likeliest component from the original data for the scan. The most probable model of the residual computed by Bayesian parameter estimation is taken as the second likeliest component of the original signal. For each scan, the waveform of the second likeliest component is a non-chirped exponentially rising sinusoid. This appears to be a reflection of an exponentially decaying sinusoidal component off the back of the workpiece. As shown in Table 1, the second likeliest component contains approximately $2-3 \%$ of the signal energy for each scan. 

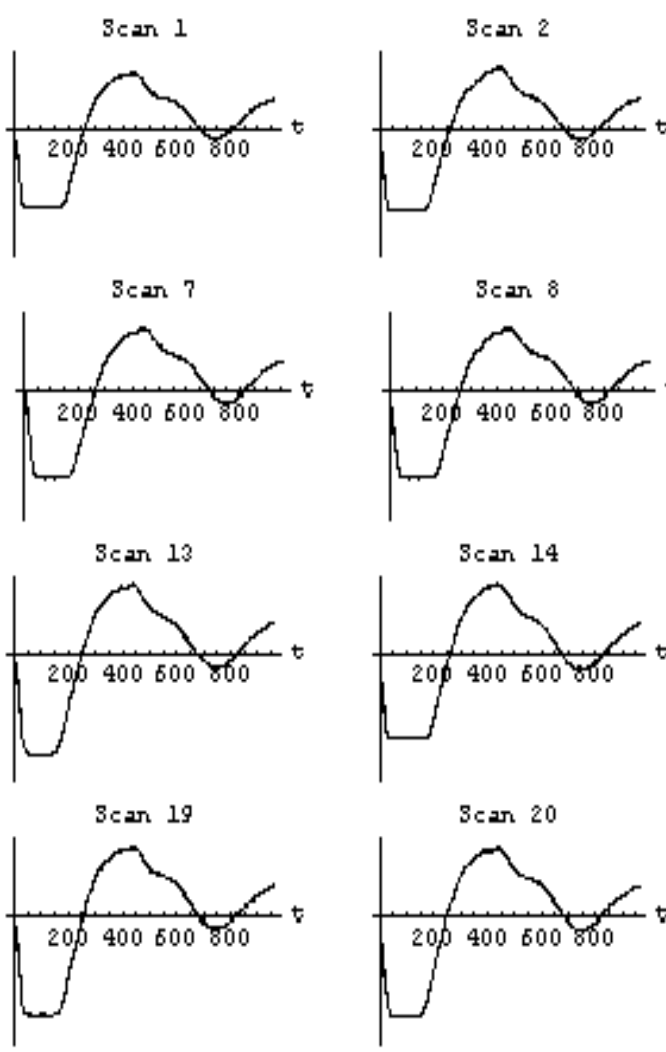

$3 \cos 25$

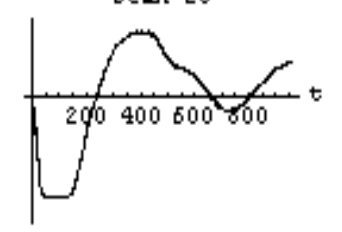

$3 \cos 26$

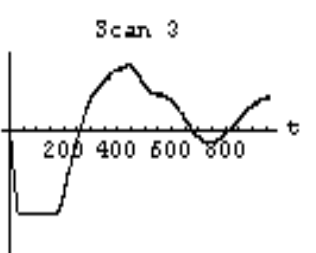

$\operatorname{sen} 9$
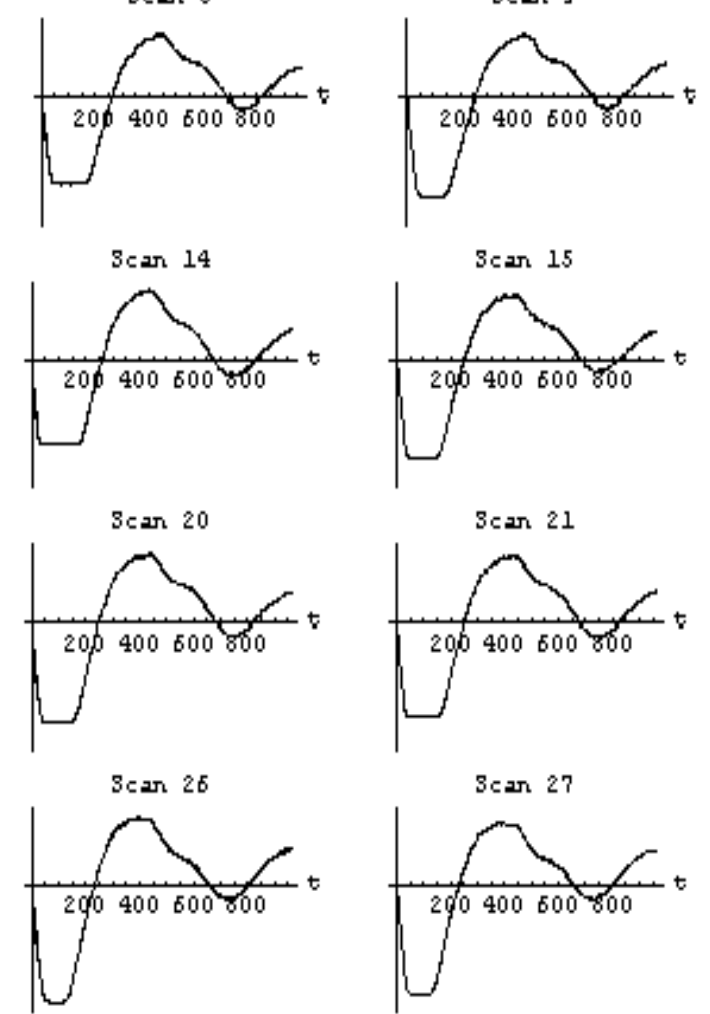

$3 \cos 15$
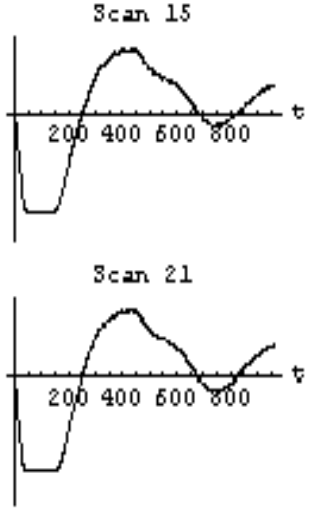

$3 \cos 27$

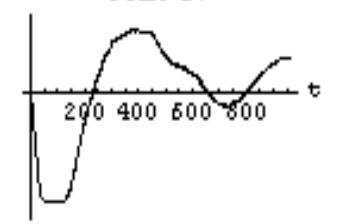

Figure 13. Successive Scans Across a Flawed Weld.

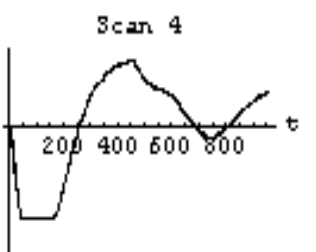

$\operatorname{sen} 10$
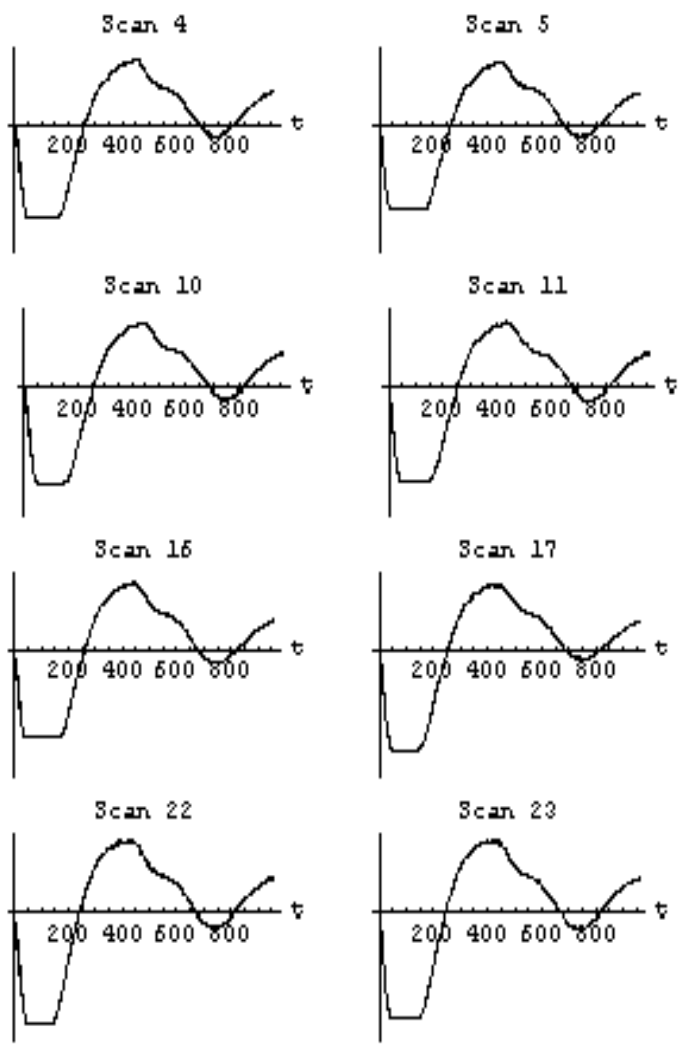

$3 \cos 28$

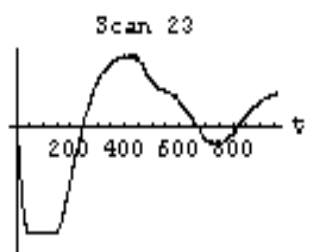

$3 \cos 29$

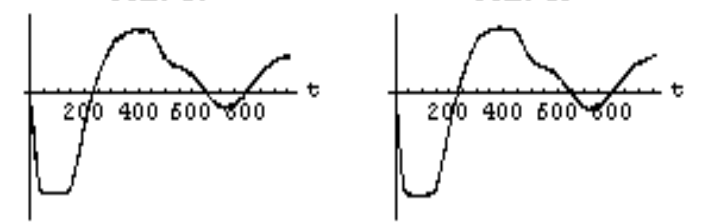

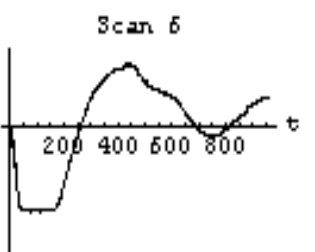

$\operatorname{sen} 12$
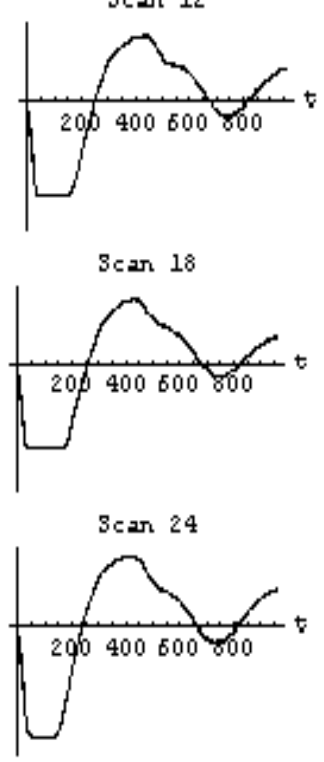

$3 \cos 30$

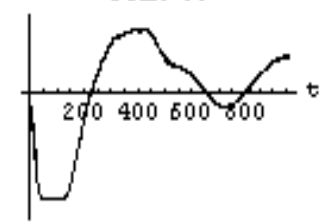



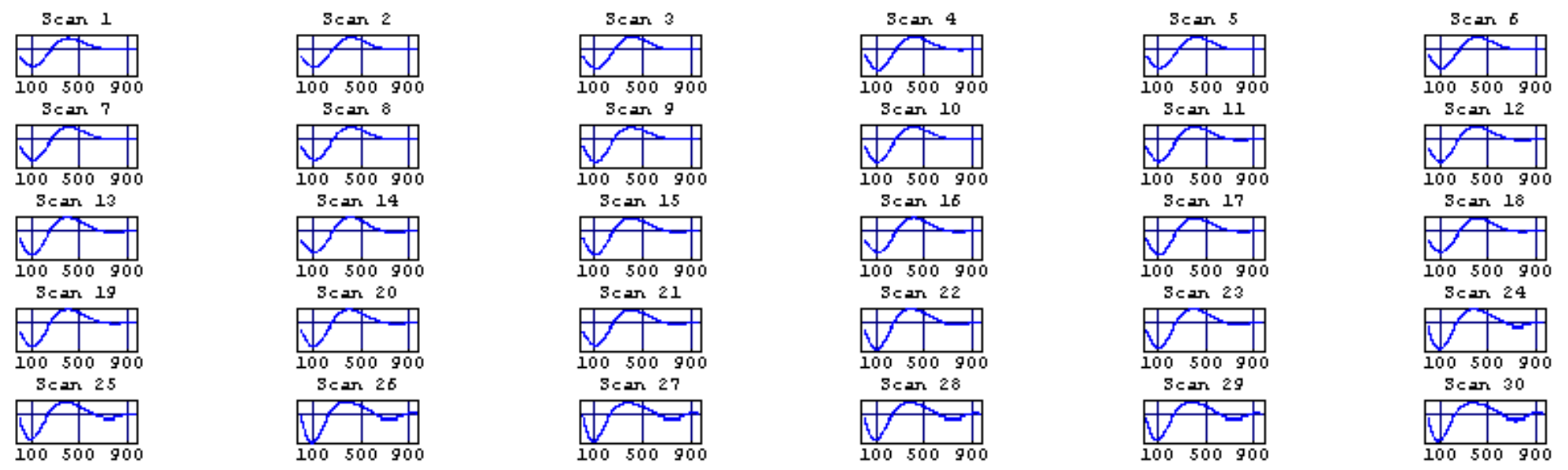

Figure 14. Likeliest Component of Each Scan.
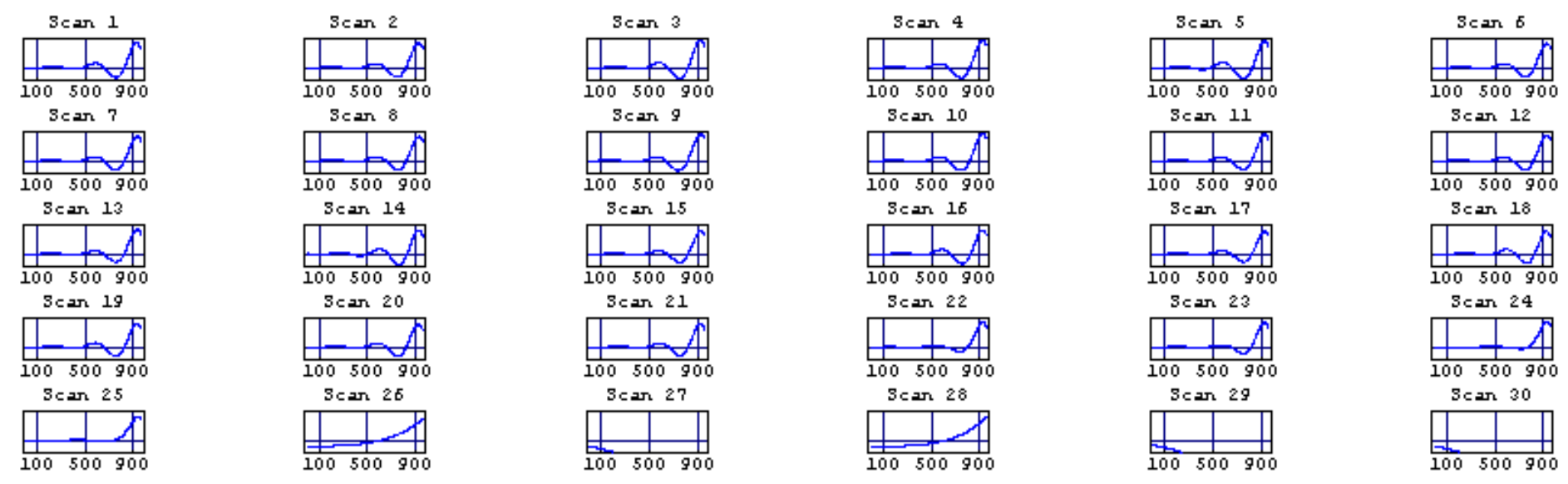

Figure 15. Second Likeliest Component of Each Scan. 

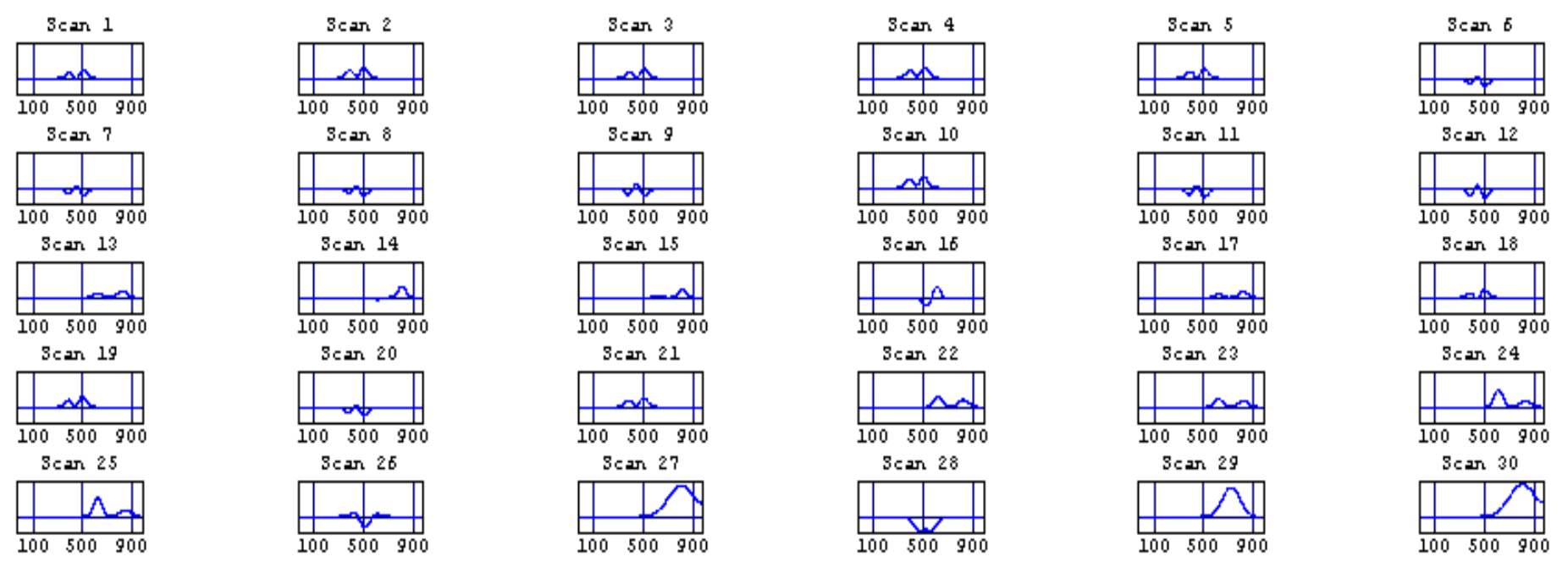

Figure 16. Third Likeliest Component of Each Scan.

Table 1. Percentage of Energy in Three Likeliest Components of Each Scan

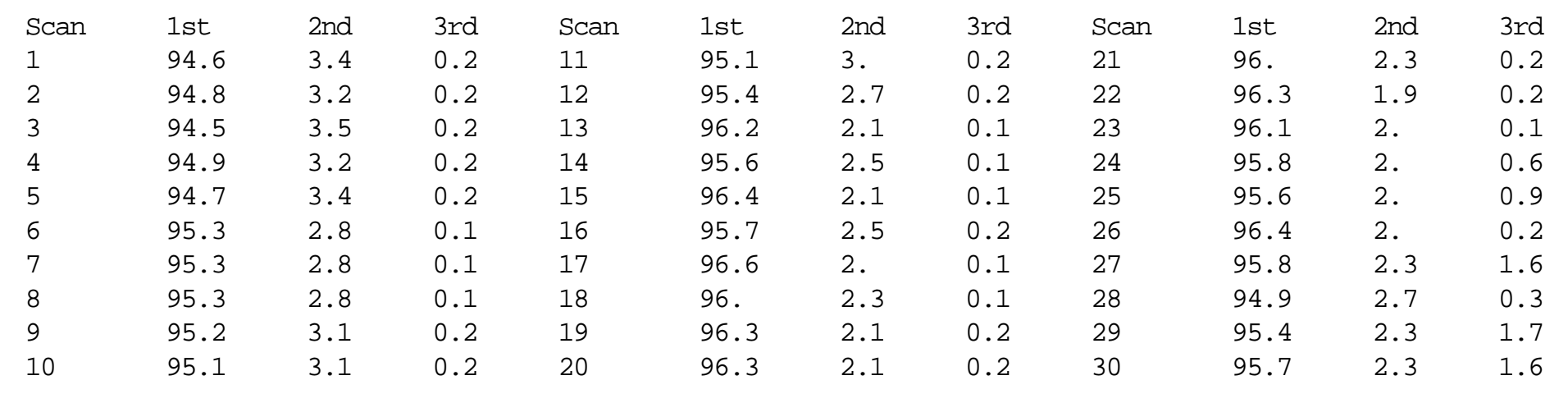


The third likeliest component for the data of each of the 30 scans is plotted in Figure 16. For each scan, the second residual is computed by subtracting the estimated data for the second likeliest component from the first residual for the scan. The most probable model of the second residual computed by Bayesian parameter estimation is taken as the third likeliest component of the original signal. For each scan, the waveform of the third likeliest component is the sum of several Gaussian pulses. This appears to be a reflection off the weld. As shown in Table 1, the third likeliest component typically contains less than $1 \%$ of the signal energy for each scan.

Figure 16 reveals some interesting information about the workpiece. For the first 21 scans, the third likeliest component is located consistently (except for scans 14 and 15) in the neighborhood of time $=500$. This is consistent with a visually detectable pinhole flaw in the weld in the vicinity of scan 14. The inconsistent location of the third likeliest component in scans 22 through 30 suggests other flaws in the workpiece that are not revealed by visual inspection.

\section{PRELIMINARY RESULTS - DISCRETE WAVELETS}

The Bayesian parameter estimation algorithm has the disadvantage of comparatively high computational complexity. For an on-line real-time system, it is desirable to find an algorithm that gives performance that is almost as good as Bayesian, but with substantially lower computational cost. For example, discrete wavelet processing is almost as good as Bayesian for detecting short bursts and, being about three orders of magnitude faster than Bayesian, is fast enough to be implemented on a presently available digital signal-processing chip. For a given setup, the real-time algorithm might be wavelet-based, and the Bayesian analysis might be performed only occasionally, to verify calibration or to provide other guidance on how to exploit the wavelet output.

For these data, the tendency of the signal energy to concentrate in relatively few wavelet basis functions is shown in Table 2. Discrete wavelet transforms were computed of each of the 30 scans, using the 10-coefficient Daubechies least-asymmetric wavelet. For the first scan, the 8 largest wavelet coefficients contain $97 \%$ of the signal energy. The reason for asking which wavelet coefficients contain $97 \%$ of the signal energy is that the Bayesian analysis suggests that $97 \%$ of the signal energy is concentrated in the two likeliest components of the signal, and that these two components do not contain information about the weld. If we can find a cheap method

of identifying this part of the signal, we can discard it with reasonable expectation that we are not discarding very much of the useful information in the signal. For the same scan, the 52 largest wavelet coefficients contain $99.99 \%$ of the signal energy. As shown in Table 1, other scans show a similar, but not identical energy distribution. 


\section{Table 2. Percentage of Energy in Largest Wavelet Basis Functions of Each Scan}

$\begin{array}{ccccccccc}\text { Scan } & 97 \% & 99.99 \% & \text { Scan } & 97 \% & 99.99 \% & \text { Scan } & 97 \% & 99.99 \% \\ 1 & 8 & 52 & 11 & 7 & 60 & 21 & 7 & 61 \\ 2 & 8 & 58 & 12 & 7 & 68 & 22 & 7 & 57 \\ 3 & 8 & 53 & 13 & 7 & 62 & 23 & 7 & 66 \\ 4 & 7 & 56 & 14 & 8 & 54 & 24 & 8 & 51 \\ 5 & 8 & 62 & 15 & 7 & 63 & 25 & 8 & 62 \\ 6 & 7 & 49 & 16 & 7 & 53 & 26 & 8 & 69 \\ 7 & 7 & 49 & 17 & 7 & 54 & 27 & 8 & 60 \\ 8 & 7 & 49 & 18 & 7 & 51 & 28 & 8 & 61 \\ 9 & 7 & 56 & 19 & 7 & 62 & 29 & 8 & 57 \\ 10 & 7 & 53 & 20 & 7 & 58 & 30 & 8 & 65\end{array}$

This energy distribution is consistent with what was gleaned from the Bayesian analysis and provides a convenient way to parse the signal to eliminate irrelevant information. The largest 7 or 8 wavelet coefficients contain the $97 \%$ of the signal energy that does not include information about the weld. The largest 60 (or thereabouts) wavelet coefficients contain in excess of 99.99\% of the total signal energy. This suggests that we can zero out the other 900 smallest and the 7 or 8 largest wavelet coefficients in each signal with practically no loss of information. The information that we seek resides in the part of the signal that remains.

Figure 17a was constructed as follows. The wavelet transform was computed for each signal. In wavelet space, the largest coefficients containing the first $99.99 \%$ of the signal energies were retained, and the others zeroed out. Then each zeroed-out set was inverse wavelet transformed to recover the approximate time series. Then each time series was subtracted from the corresponding original time series. The resulting residuals are plotted below as a density plot. The vertical axis corresponds to the 30 spatial locations on the workpiece. The horizontal axis corresponds to time. The gray level is strength. The residuals are mostly (but not entirely) noise.

In wavelet space, the 8 largest coefficients (containing 95-96\% of the signal energies) were retained, and the others zeroed out. Then each zeroed-out set was inverse wavelet transformed to recover the approximate time series. Then each time series was subtracted from the corresponding original time series. The resulting 4-5\% residuals are contour-plotted below in Figure $17 b$.

In the same wavelet space, the 9 largest coefficients (containing 96-97\% of the signal energies) were retained, and the others zeroed out. Then each zeroed-out set was inverse wavelet transformed to recover the approximate time series. Then each time series was subtracted from the corresponding original time series. The resulting 3-4\% residuals are contour-plotted in Figure 17c. Note that there is a little difference between Figure 17b and Figure 17c.

The reasonable place to search for features of weld defects is in the region of the signal between the bias (biggest eight coefficients of each signal, or thereabouts) and the noise (smallest 900 
coefficients of each signal). In Figure $17 d, 97.6 \%$ of the signal energy is assumed to be attributed to biasing effects. This bias is subtracted from the signal approximation constructed from the wavelet coefficients constituting of $97.8 \%$, of the signal energy. This difference constitutes $1.2 \%$ of the original signal energy, and has a fairly dramatic global minimum whose contour is plotted in Figure 17d. This shows up in scan 14, and time 750. This corresponds to the change in the third likeliest component of the signal as revealed by Bayesian analysis, and to the pinhole defect in the weld in the workpiece.
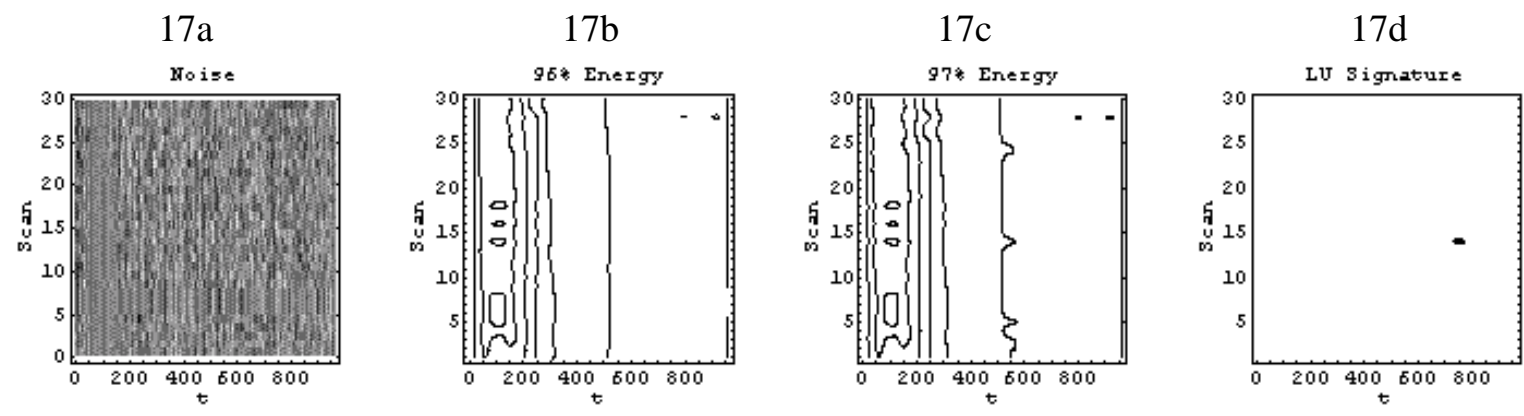

Figure 17. Separation of Signal Energies by Wavelets.

It is noteworthy that a similar strategy of wavelet-based feature extraction was independently discovered by Legendre, et al. [13]. Their problem was the extraction of Lamb wave features from EMAT data. Their fundamental strategy was, as ours is here, to use the concepts developed by Donoho [14] to subtract out irrelevant features in both the fine and coarse scales of a discrete wavelet space, so that the residual would be dominated by flaw features. Their pattern recognition strategy differs from ours in that they used the recovered flaw features to obtain an image, while we use the data in the residual to obtain a numerical feature vector.

The compression effect is also the basis for Donoho denoising. Donoho says that for a wavelet transform of a noisy signal, the big coefficients hold the information and the small coefficients hold the noise. Since the wavelet compresses the information, a signal spread out in the time domain will be very compactly represented in the wavelet domain. On the other hand, noise is approximately evenly randomly distributed throughout both the wavelet and the time domains. The problem with Donoho's technique is that it is a bit of an art to decide where to draw the line between big coefficients and small ones.

Suppose we try to denoise with the best basis wavelet packet, by declaring that the biggest coefficients holding $90 \%$ of the total energy of the signal constitute the "information." In the left-hand plot of Figure 18, the jagged line is the original time series. The smooth line is constructed by taking the wavelet packet transform and identifying and retaining the 9 coefficients that hold $90 \%$ of the energy in transform space, and zeroing-out the 951 coefficients that contain the other $10 \%$ of the energy. The "denoised" time series is recovered by taking the inverse wavelet packet transform of the zeroed-out list. It is noted that the high frequency features of the time domain signal are mostly preserved (unlike the more traditional method of denoising by low-pass filtering), and noise is substantially reduced. However, it appears that there is some information being lost when we discard that last $10 \%$ of the energy. 
A similar analysis was done by taking the wavelet packet transform and identifying and retaining the 35 coefficients that hold $98 \%$ of the energy in transform space, and zeroing-out the 925 coefficients that contain the other $2 \%$ of the energy. The "denoised" time series is recovered by taking the inverse wavelet packet transform of the zeroed-out list. In going from $90 \%$ to $98 \%$ of the signal energy (using the next 26 wavelet packet coefficients) it does not look at first glance that the next 26 coefficients bought us much.

The signal shown in Figure 18 is the $22^{\text {nd }}$ of a set of 26 time series collected for a particular specimen.

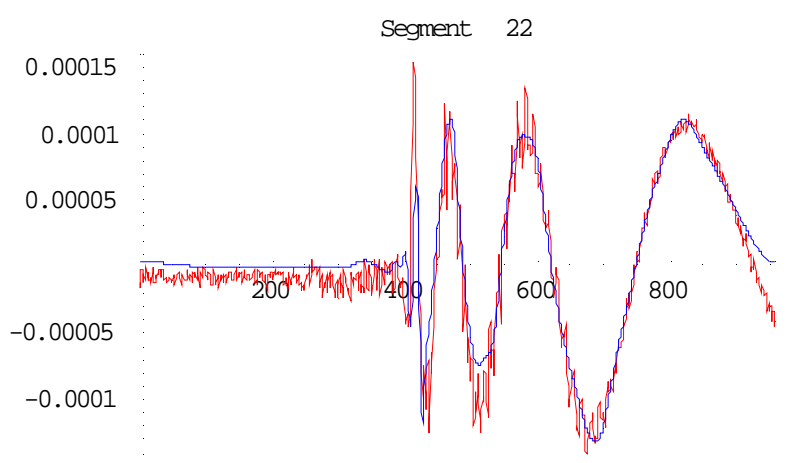

Figure 18. Denoising a Laser Ultrasonic Signal.

\section{THE OBSERVED EFFECT OF WELD FLAWS ON LAMB WAVE MODES}

The utility of a commercial weld flaw detector depends on its ability to consistently detect the signatures of different weld types. Earlier research on this project showed that entangled Lamb wave modes could be readily separated by Bayesian parameter estimation. The objective of the latter part of the research in this project has been to investigate the effect of specific weld flaws on the mode structure in the expectation that a specific mode structures can be associated with specific types of weld flaws.

In early 2000, Lasson Technologies collected the sample 11 and sample 15 data sets in their laboratory. Sample 11 is a Tailor-welded blank with a good weld with low porosity. Sample15 is a tailor-welded blank that is known to have high porosity from X-ray data.

For both samples, the Lamb waves were generated by firing an excitation laser through linefocused optics at the thin part of the sample. The receive optics used a point detection beam on the thick part of the sample. The spacing between generation and detection beams was about $42 \mathrm{~mm}$. The weld was between the detection and generation beams.

For both samples, what is actually being studied is the effect of the weld on the Lamb wave as it passes through the weld on a straight-line path from the excitation line to the receiving spot. To 
observe the variation along the weld, the excitation and receive optics are scanned along the weld. In practice the scanning is done by holding the optics fixed, and moving the sample along the axis of the weld between successive scans. For sample 11, waveforms were collected for 55 scans. For sample 15, waveforms were collected for 52 scans. The samples were moved by $0.5 \mathrm{~mm}$ between scans.

The model of each Lamb wave mode is assumed to be a Gaussian-windowed chirped sinusoid of the form

$A e^{\left(\frac{\mathrm{t}-\tau}{\sigma}\right)^{2}} \cos \left[\alpha(t-\tau)^{2}-(t-\tau) \omega\right]+B e^{\left(\frac{\mathrm{t}-\tau)^{2}}{\sigma}\right.} \sin \left[\alpha(t-\tau)^{2}-(t-\tau) \omega\right]$

where

$\mathrm{t}=$ the independent variable, and the parameters are

$\mathrm{A}$ and $\mathrm{B}=$ the amplitudes of the two components

$\tau=$ pulse group delay

$\sigma=$ pulse width

$\omega=$ center frequency of oscillation

$\alpha=$ chirp rate

Each mode consists of a wave of this kind with a different set of values for the parameters.

For each scan of both samples, the parameters were estimated for the most likely modes. For each mode, the log likelihood (LL) is taken as a figure of merit. The greater the LL, the more likely the mode is present. For each mode, the "\% erg" is the energy in the mode divided by the total energy of the signal. The results of the Bayesian analysis are tabulated in Appendix A.

The more pressing issue is, do all these numbers actually convey any meaning? To appreciate what they tell us about the process, consider the following. Figure 19 is a plot of sample 15 scan

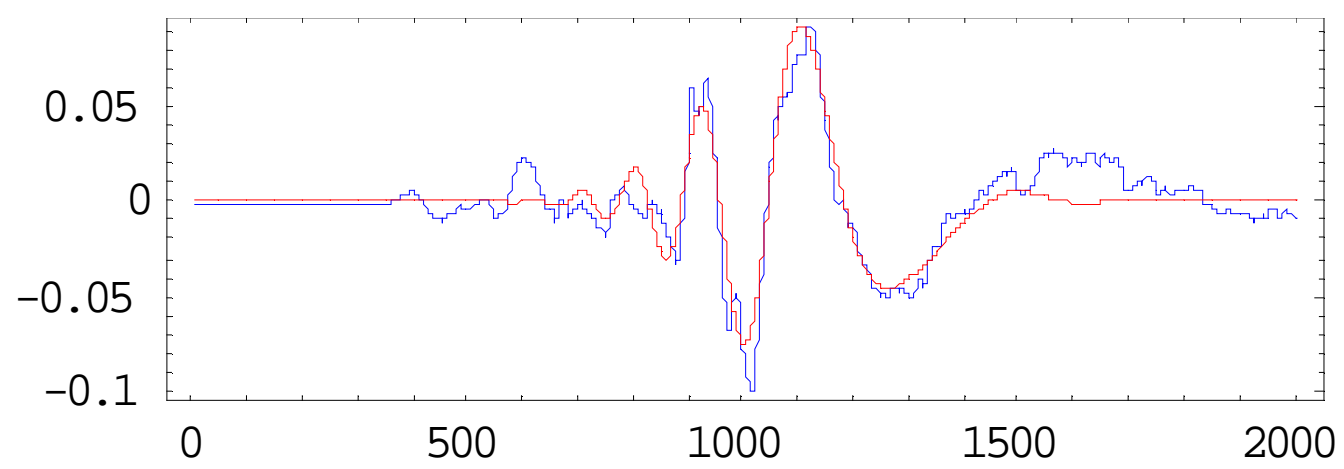

Figure 19. Sample 15-Scan 1. 
1. Blue is the raw data with the DC bias subtracted out. Red is the mode 1 component, obtained from Bayesian parameter estimation. The plots show signal intensity versus time.

For reliable pattern recognition, the relevant question is, do any of the parameters tend to cluster in an isolated region of parameter space? In particular, are the clusters well separated by class? What is desired in this case is a parameter value that is distinctly different for the class of highporosity welds, and the class of good welds.

In these observations, the fifth likeliest mode showed the strongest tendency to form distinct clusters. The fifth mode for the good welds tend to look very similar to each other. The fifth mode for the high-porosity welds tend to look very similar to each other. However, the fifth modes for the two classes tend not to look like each other.

Figure 20 is a plot of the Gaussian Distributions based on the means and standard deviations of the dominant frequency of mode 5. Blue is Sample 15. Red is sample 11. The frequencies of mode 5 tend to cluster, but the cluster for sample 11 does not appear to be especially well separated from the cluster for sample 15.

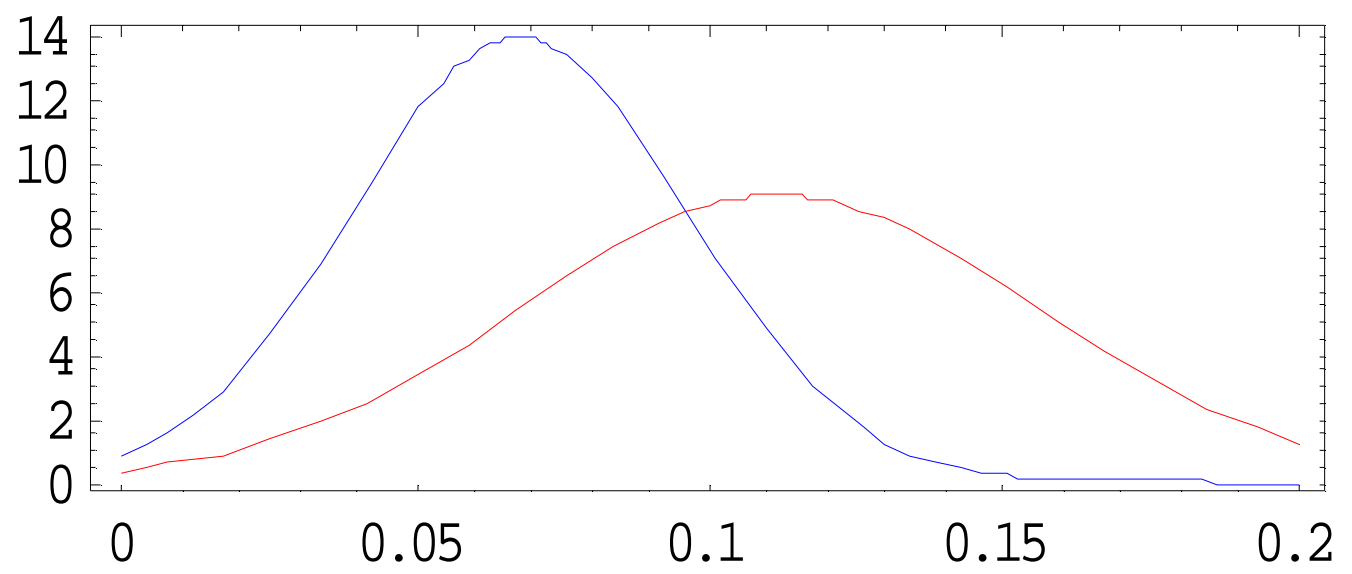

Figure 20. Distribution of Frequencies in Mode 5. 
Can we improve the classification by exploiting coincidence between these two parameters? If both the concentrations of energy in mode 1 (Figure 21) and the frequencies in mode 5 are caused by the difference in porosity of the samples, then we would expect there to be an exploitable coincidence between the two features. If mode 1 has a high concentration of energy, AND mode 5 has a low frequency, then it is much more likely that the signature is that of a highporosity weld than that of a good weld.

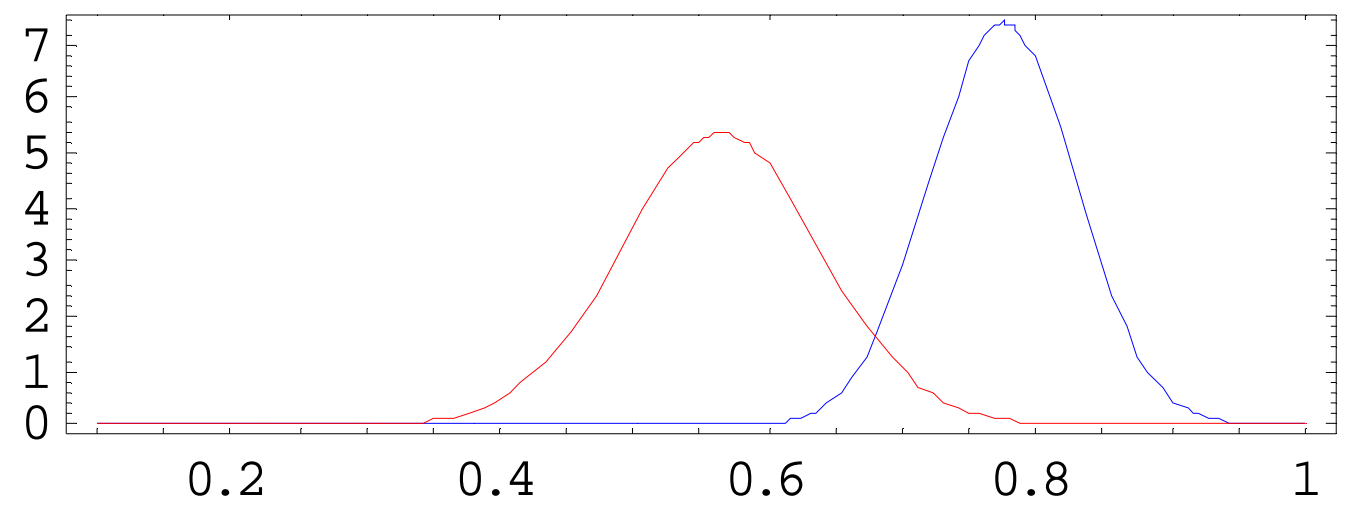

Figure 21. Distribution of \% Energies in Mode 1.

Suppose that each scan of each sample is characterized by a feature vector or ordered pair consisting of the frequency of mode 5 and the $\%$ energy of mode 1 . Figure 22 is the plot of the ordered pairs for both samples. Red is sample 15. Blue is sample 11. Horizontal axis is frequency of mode 5 . Vertical axis is $\%$ energy in mode 1 . A possible decision criterion would be that we could detect high porosity if we consider all the scans of a sample and observe if there were any scans with \% energy in mode 1 above $78 \%$, AND with frequency of mode 5 below $0.035 \mathrm{radians} / \mathrm{sec}$.

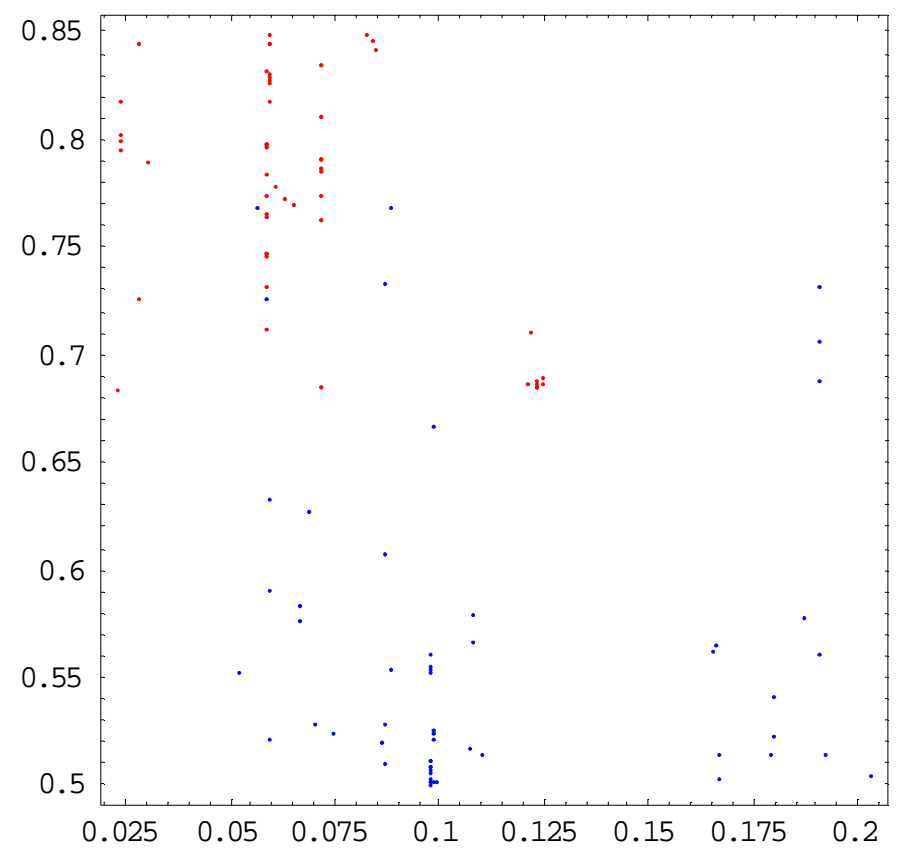

Figure 22. Weld Features In 2D Feature Space. 
The quartile ellipses show the comparative compactness (or lack thereof) of the clusters. They are centered on the centroids of the sample lists for each class. The innermost ellipse encloses $25 \%$ of the samples of the class. The middle ellipse encloses $50 \%$ of the samples of the class. The innermost ellipse encloses $75 \%$ of the samples of the class. In Figure 23, we see the quartile ellipses superimposed over the 2D features. Notice that the 75 th percentile ellipse for each class is outside the 75 th percentile ellipse for the other. Thus, we are reasonably well separated. If we found yet another coincident parameter in the data, we could probably improve the separation of the classes further.

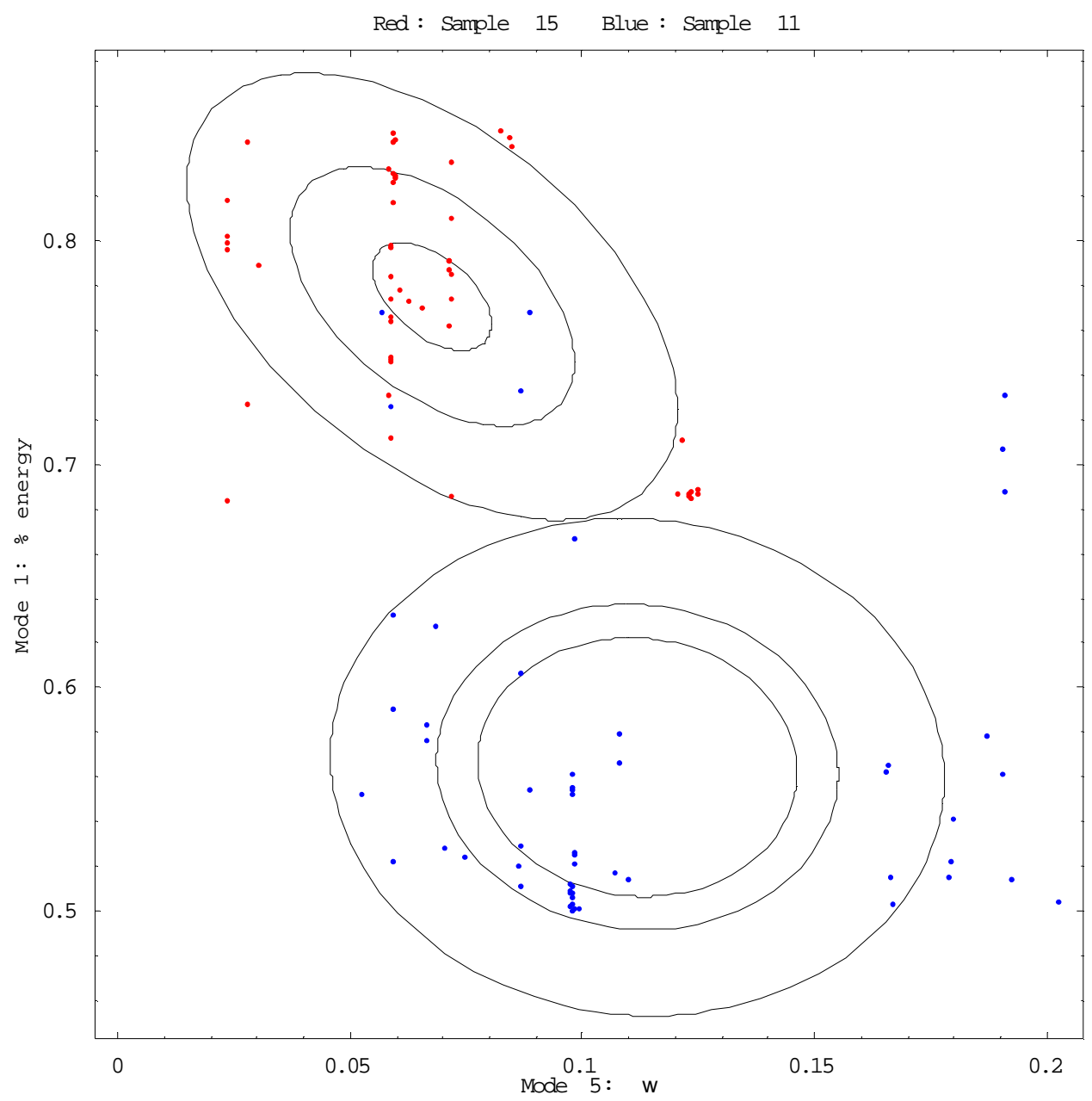

Figure 23. Quartile Ellipses for the Two Classes in Feature Space.

The concept illustrated by the quartile ellipses is equivalent to the idea of Mahalanobis distance. Suppose that $\mathrm{x}$ is an $\mathrm{n}$-dimensional feature vector, and that a given class is defined by a cluster of n-dimensional feature vectors, whose mean (or centroid) is mu, and whose covariance matrix is $\mathrm{cv}$. The Mahalanobis distance from sample $\mathrm{x}$ to the cluster is given by the following formula. 


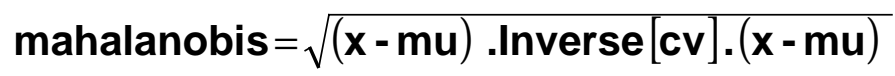

The practical meaning of the Mahalanobis distance is as follows. The location of the cluster center is its centroid. Any given sample of the cluster has some probability of being a member of the cluster. The surfaces of constant probability are given by a family of hyper-ellipsoids centered on the centroid. The eigenvectors of the covariance matrix of the cluster point in the directions of the axes of the hyper-ellipsoid. The ratios of the lengths of the eigenvectors are the same as the ratios of the lengths of the axes of the hyper-ellipsoid. Thus, the covariance matrix and centroid define the location, orientation, and shape of the entire family. The Mahalanobis radius is the single parameter that defines the size of the surface of constant probability, or the distance from the cluster center.

\section{CONCLUSIONS AND FURTHER RESEARCH}

The authors have detected various kinds of weld flaws in various specimens of tailor-welded blanks using the methods described in this paper. These results demonstrate capability to detect localized weld defects using a computationally efficient processing approach that can be implemented in real-time at the frequencies at which these signatures occur. In ongoing research we are identifying the connection between the Bayesian-derived models and the underlying physical processes. We will also investigate the comparative reliability and computational cost of wavelet and Bayesian feature extraction methods.

In future work we will seek to classify individual defects, including those encountered in the welding process. We will also devise a practical implementation of the signal-processing algorithm in real time. The ultimate goal of this research is to construct a prototype of an on-line LBU weld inspection device for tailor-welded blanks.

This work could lead to spin-offs for other on-line inspection in other processes. Additional follow-on research might include the examination of other types of continuous seam joints for distinguishing features. In addition, the Lamb-wave modeling and feature extraction would be directly applicable to inspection of other products fabricated from thin metal sheet.

Bayesian parameter estimation is able to separate entangled Lamb wave modes. Pattern recognition algorithms applied to Lamb mode features have produced robust features for distinguishing between several types of weld defects. In other words, the information is present in the output of the laser ultrasonic hardware, and it is feasible to extract it. Wavelet analysis produces results that are almost as good as Bayesian, but execute a thousand times faster. This study demonstrates the principle that it is feasible to construct a laser ultrasonic system to detect weld defects in thin metal parts on-line in real-time, and to classify the defects according to type. 
This page left intentionally blank 


\section{ACKNOWLEDGMENTS}

This research is a joint effort between Lasson Technologies, TWB, Inc, and the US Department of Energy, and is sponsored under ER-LTR CRADA ORNL 98-0524. 
This page left intentionally blank 


\section{REFERENCES}

1. Klein, M.B., Dunning, G.J., Mitchell, P.V., O'Meara, T.R., and Owechko, Y., "Remote Laser-Based Ulrasonic Inspection of Weld Joints for High Volume Industrial Applications," in Review of Progress in Quantitative Nondestructive Evaluation, D.O Thompson and D.E. Chimenti, Editors, Volume 15, pp. 2257-2264, (1996).

2. Bussiere, J., McQueen, S., Shannon, R., Spicer, J., and Russo, R., Report of the Workshop on Industrial Applications of Laser Ultrasonics, produced by US Department of Energy Office of Industrial Technologies, pp. 30-32, draft January 1998.

3. Kercel, S.W., Labaj, L.E., and Baylor, V.M, "Comparison of Enclosed Space Detection System with Conventional Methods," American Defense Preparedness Association, Proceedings of 13th Annual Security Technology Symposium, Virginia Beach VA, June 9-12, (1997).

4. Kercel, S.W., Dress, W.B., Hibbs, A.D., and Barrall, G.A, "Investigation of Wavelet-Based Enhancements to Nuclear Quadrupole Resonance Explosives Detectors," in Wavelet Applications IV, Harold H. Szu, Editor, Proc. SPIE 3391, pp. 424-434, (1998).

5. Dress, W.B. and Kercel, S.W., "Wavelet-Based Acoustic Recognition of Aircraft," in Wavelet Applications, Harold H. Szu, Editor, Proc. SPIE 2242, pp. 778-791, (1994).

6. Simpson, W.A. and McGuire, D.J., Phase and Group Velocities for Lamb Waves in DOP26 Iridium Alloy Sheet, ORNL/TM-12749, July 1994.

7. Bretthorst, L., Bayesian Spectrum Analysis and Parameter Estimation, pp. 1-5, SpringerVerlag, Berlin, 1988.

8. Kercel, S.W., A Near-Real-Time Instrument for Wideband Magnetic Field Monitoring with Simultaneous Time and Frequency Localization by Multiresolution Filter Bank, pp. 109-113, UMI, Ann Arbor, 1996.

9. Daubechies, I., Ten Lectures on Wavelets, pp. 199-209, Society for Industrial and Applied Mathematics, Philadelphia, 1992.

10. M. V. Wickerhauser, Adapted Wavelet Analysis from Theory to Software, pp. 237-256, A. K. Peters, Ltd., Wellesley, 1994.

11. Ibid., pp, 274-281.

12. Yu He, Wavelet Explorer, pp. 136-137, Wolfram Research, Inc., Champaign, 1996. 
13. Legendre, S., Massicotte, D., Goyette, J. and Bose, T. K., "Wavelet-Transform-Based Method of Analysis of Lamb-Wave Ultrasonic NDE Signals," IEEE Transactions on Instrumentation and Measurement, Vol. 49, No. 3, June 2000, pp. 524-530.

14. Donoho, D., De-noising by Soft-thresholding, Technical Report, Department of Statistics, Stanford University, 1992. 


\section{APPENDIX A. RESULTS OF BAYESIAN ANALYSIS}

For Sample 11 the Bayesian results consists of 1100 lines 10 features per line. The 10 features are : the sample name $(\mathrm{S})$, the scan or trial number $(\mathrm{T})$, the mode number $(\mathrm{M})$,

the log likelihood (LL) of the fit of the model to the data (greater magnitude $=>$ better fit), the radian frequency $(\omega=\pi$ radians $/ \mathrm{sec} \leftrightarrow$ frequency $=50 \mathrm{MHz})$, the window width $(\sigma)$ in time bins (10 ns binwidth), the time of occurrence $(\tau)$ of the peak of the envelope in time bins, the chirp rate $(\alpha)$ in radians/sec/ sec, the fraction (\% erg) of the total energy of the signal in this scan that is contained in this mode, and the phase angle $(\phi)$ in radians of this mode. $\phi$ is determined from the ratio of the amplitudes of the two components of the model.

\section{Sample 11 Scan 1:}

\begin{tabular}{|c|c|c|c|c|c|c|c|c|c|c|}
\hline$s$ & & $\mathrm{~T}$ & M & LL & w & s & $\mathrm{t}$ & a & $\therefore$ erg & $f$ \\
\hline $\mathrm{s}$ & 11 & 1 & 1 & 891.486 & 0.0219528 & 241.34 & 1057.11 & 0.0000636014 & 0.589954 & -0.582861 \\
\hline & 11 & 1 & 2 & 218.674 & 0.00705987 & 556.862 & 1610.6 & $-2.37292^{\prime} 10^{-6}$ & 0.080417 & -0.566525 \\
\hline & 11 & 1 & 3 & 57.457 & 0.0863169 & 7.3859 & 850.281 & 0.000121015 & 0.0746554 & 1.84485 \\
\hline & 11 & 1 & 4 & 143.665 & 0.164946 & 424.604 & 1088.97 & -0.000028916 & 0.0340235 & 2.1575 \\
\hline & 11 & 1 & 5 & 62.6546 & 0.0592876 & 195.868 & 591.243 & $2.8123^{\prime} 10^{-7}$ & 0.0133745 & 0.605648 \\
\hline & 11 & 1 & 6 & 109.317 & 0.0988647 & 196.927 & 1000 . & $-1.93821^{\prime} 10^{-6}$ & 0.0177399 & -2.05683 \\
\hline & 11 & 1 & 7 & 112.632 & 0.190437 & 199.158 & 800. & $-3.0547545711078545^{\prime} 10^{-9}$ & 0.0147756 & 0.906881 \\
\hline & 11 & 1 & 8 & 88.4211 & 0.169883 & 228.483 & 834.159 & $-6.95433^{\prime} 10^{-6}$ & 0.0147299 & -0.258868 \\
\hline & 11 & 1 & 9 & 48.8999 & 0.0879672 & 790.291 & 1292.13 & $-7.37616^{\prime} 10^{-7}$ & 0.00761673 & 1.58724 \\
\hline & 11 & 1 & 10 & 127.659 & 0.207937 & 242.366 & 900.27 & $3.13609^{\prime} 10^{-6}$ & 0.0181705 & 1.04786 \\
\hline & 11 & 1 & 11 & 142.289 & 0.114265 & 251.368 & 1000 . & $5.43806^{\prime} 10^{-7}$ & 0.0112763 & -0.709451 \\
\hline & 11 & 1 & 12 & 170.002 & 0.313122 & 136.598 & 1000.01 & $-2.4176^{\prime} 10^{-7}$ & 0.0128465 & -0.792714 \\
\hline & 11 & 1 & 13 & 210.183 & 0.136972 & 226.101 & 900. & $3.1360449020868083^{\prime} 10^{-6}$ & 0.0116638 & 0.167548 \\
\hline & 11 & 1 & 14 & 72.9812 & 0.0225386 & 591.298 & 922.174 & $7.5567287681535435^{\prime} 10^{-6}$ & 0.00689933 & -1.78984 \\
\hline & 11 & 1 & 15 & 168.348 & 0.274278 & 108.632 & 900. & $8.22862^{\prime} 10^{-6}$ & 0.0100348 & 0.0536383 \\
\hline & 11 & 1 & 16 & 97.8568 & 0.0476629 & 503.066 & 1154.97 & -0.000019033 & 0.00755959 & -1.05251 \\
\hline & 11 & 1 & 17 & 178.805 & 0.234476 & 98.4364 & 1200. & $2.88116^{\prime} 10^{-6}$ & 0.0115066 & 1.78805 \\
\hline & 11 & 1 & 18 & 87.0257 & 0.19791 & 313.6 & 1413.97 & -0.000020744 & 0.00516971 & -3.03261 \\
\hline & 11 & 1 & 19 & 90.9517 & 0.200192 & 882.212 & 2007.85 & -0.000126249 & 0.00494305 & -2.07839 \\
\hline & 11 & 1 & 20 & 90.7236 & 0.155374 & 182.522 & 1100. & $3.1441697047851137^{\prime} 10^{-6}$ & 0.00279217 & 2.4714 \\
\hline
\end{tabular}

Sample 11 Scan 2:

\begin{tabular}{|c|c|c|c|c|c|c|c|c|c|}
\hline $\mathrm{s}$ & $\mathrm{T}$ & M & LL & w & s & $\mathrm{t}$ & a & $\therefore$ erg & $f$ \\
\hline S 11 & 2 & 1 & 875.162 & 0.0222129 & 239.834 & 1054.73 & 0.0000639627 & 0.583206 & -0.547991 \\
\hline S 11 & 2 & 2 & 206.9 & 0.00704514 & 554.066 & 1605.91 & $-2.3222365993759063^{\prime} 10^{-6}$ & 0.0777925 & -0.543461 \\
\hline S 11 & 2 & 3 & 163.619 & 0.0854657 & 225.631 & 904.618 & $-8.07172^{\prime} 10^{-6}$ & 0.0370696 & -2.2369 \\
\hline S 11 & 2 & 4 & 204.923 & 0.106506 & 221.458 & 1099.79 & 0 . & 0.0374659 & 0.546041 \\
\hline S 11 & 2 & 5 & 103.584 & 0.0666278 & 337.586 & 728.358 & -0.0000130975 & 0.0259576 & -2.02303 \\
\hline S 11 & 2 & 6 & 152.904 & 0.165477 & 422.429 & 1096.91 & -0.0000285018 & 0.0337228 & 0.816999 \\
\hline S 11 & 2 & 7 & 81.1464 & 0.190468 & 209.481 & 799.977 & $-6.01935^{\prime} 10^{-9}$ & 0.0157097 & 0.930013 \\
\hline S 11 & 2 & 8 & 79.226 & 0.0999091 & 551.028 & 1196.74 & $-6.78192^{\prime} 10^{-6}$ & 0.0143507 & -0.961162 \\
\hline S 11 & 2 & 9 & 89.0345 & 0.171874 & 247.926 & 899.873 & $-2.5722346843080974^{\prime} 10^{-6}$ & 0.0148067 & 1.00869 \\
\hline S 11 & 2 & 10 & 123.398 & 0.207953 & 235.974 & 900. & $3.5947655858951914^{\prime} 10^{-10}$ & 0.017996 & 1.13307 \\
\hline S 11 & 2 & 11 & 185.191 & 0.136287 & 189.845 & 900.016 & $1.6388883613977106^{\prime} 10^{-7}$ & 0.0159408 & 0.223874 \\
\hline S 11 & 2 & 12 & 175.676 & 0.3135 & 138.884 & 1000 . & $2.26329^{\prime} 10^{-6}$ & 0.0135246 & -0.792616 \\
\hline S 11 & 2 & 13 & 68.2568 & 0.048885 & 518.678 & 1043.38 & $8.6271^{\prime} 10^{-7}$ & 0.0073757 & -2.60597 \\
\hline S 11 & 2 & 14 & 75.9162 & 0.109453 & 348.893 & 895.46 & $-7.7246^{\prime} 10^{-6}$ & 0.00763316 & 2.9412 \\
\hline S 11 & 2 & 15 & 76.5547 & 0.0235208 & 376.343 & 903.712 & 0.0000112768 & 0.00713239 & -1.60665 \\
\hline S 11 & 2 & 16 & 222.467 & 0.274391 & 169.895 & 900. & $5.14194^{\prime} 10^{-6}$ & 0.0104972 & 0.0753802 \\
\hline S 11 & 2 & 17 & 172.393 & 0.234833 & 102.468 & 1200. & $-1.1723828379304497^{\prime} 10^{-6}$ & 0.0121616 & 1.74657 \\
\hline S 11 & 2 & 18 & 85.5987 & 0.198417 & 456.314 & 1405.92 & -0.0000207564 & 0.0054955 & -1.41224 \\
\hline S 11 & 2 & 19 & 77.6799 & 0.157631 & 307.009 & 1016.59 & $-5.46622^{\prime} 10^{-6}$ & 0.00459589 & 2.83812 \\
\hline S 11 & 2 & 20 & 90.9174 & 0.0281614 & 342.053 & 1448.88 & 0.0000195685 & 0.00494468 & 1.84 \\
\hline
\end{tabular}




\section{Sample 11 Scan 3:}

\begin{tabular}{llllll} 
S & T & $M$ & \multicolumn{1}{c}{ LL } & \multicolumn{1}{c}{ W } \\
$S$ & 11 & 3 & 1 & 864.81 & 0.0224522 \\
$S$ & 11 & 3 & 2 & 197.916 & 0.00704393 \\
$S$ & 11 & 3 & 3 & 479.738 & 0.0850278 \\
$S$ & 11 & 3 & 4 & 131.825 & 0.165495 \\
$S$ & 11 & 3 & 5 & 176.899 & 0.108066 \\
$S$ & 11 & 3 & 6 & 120.065 & 0.190349 \\
$S$ & 11 & 3 & 7 & 136.653 & 0.20762 \\
$S$ & 11 & 3 & 8 & 90.917 & 0.169575 \\
$S$ & 11 & 3 & 9 & 105.804 & 0.137045 \\
$S$ & 11 & 3 & 10 & 99.1462 & 0.0649509 \\
$S$ & 11 & 3 & 11 & 178.749 & 0.313707 \\
$S$ & 11 & 3 & 12 & 68.7003 & 0.02272 \\
$S$ & 11 & 3 & 13 & 170.528 & 0.274324 \\
$S$ & 11 & 3 & 14 & 98.2679 & 0.0476843 \\
$S$ & 11 & 3 & 15 & 165.447 & 0.23459 \\
$S$ & 11 & 3 & 16 & 84.592 & 0.0939662 \\
$S$ & 11 & 3 & 17 & 80.6218 & 0.198137 \\
$S$ & 11 & 3 & 18 & 78.7497 & 0.107685 \\
$S$ & 11 & 3 & 19 & 25.7424 & 0.219367 \\
$S$ & 11 & 3 & 20 & 77.4835 & 0.0323452
\end{tabular}

\section{Sample 11 Scan 4:}

\begin{tabular}{|c|c|c|c|c|}
\hline $\mathrm{s}$ & $\mathrm{t}$ & a & $\frac{\circ}{6}$ erg & $f$ \\
\hline 239.674 & 1053.09 & 0.0000641533 & 0.578868 & -0.517153 \\
\hline 552.938 & 1603.96 & $-2.37346^{\prime} 10^{-6}$ & 0.0755154 & -0.528665 \\
\hline 100.118 & 838.617 & $-5.69346^{\prime} 10^{-6}$ & 0.0709824 & 3.05567 \\
\hline 425.186 & 1097.52 & -0.0000285818 & 0.0338351 & 0.723567 \\
\hline 112.682 & 1100 & $1.6267855233153072^{\prime} 10^{-7}$ & 0.0323898 & 0.306036 \\
\hline 207.893 & 800.003 & $6.36962^{\prime} 10^{-6}$ & 0.016377 & 0.850507 \\
\hline 224.963 & 900. & 0 . & 0.0206027 & 1.12449 \\
\hline 229.227 & 836.227 & $-7.1273151877059466^{\prime} 10^{-6}$ & 0.0148312 & -0.6037 \\
\hline 240.042 & 900. & $-5.09148^{\prime} 10^{-6}$ & 0.0155157 & 0.186899 \\
\hline 131.696 & 579.109 & $-8.4597^{\prime} 10^{-6}$ & 0.0121572 & 1.26583 \\
\hline 130.845 & 1000 & $-2.0810252131660294^{\prime} 10^{-6}$ & 0.0137742 & -0.754415 \\
\hline 549.2 & 919.225 & $8.10274^{\prime} 10^{-6}$ & 0.00760025 & -1.79851 \\
\hline 168.443 & 900. & $3.1298460828493087^{\prime} 10^{-6}$ & 0.0104969 & 0.141136 \\
\hline 423.717 & 1142.91 & -0.0000169463 & 0.00902017 & -0.485038 \\
\hline 97.6394 & 1199.77 & $5.07431^{\prime} 10^{-6}$ & 0.0128109 & 1.77158 \\
\hline 544.693 & 1244.11 & 0.0000161451 & 0.00604652 & -0.349719 \\
\hline 316.251 & 1424.83 & -0.0000215143 & 0.00530568 & 1.10376 \\
\hline 731.723 & 1553.74 & 0.0000300183 & 0.00478547 & -0.250127 \\
\hline 1264.24 & 1526.96 & $-5.89181^{\prime} 10^{-7}$ & 0.00148433 & 1.57728 \\
\hline 544.689 & 1436.73 & 0.0000352199 & 0.00424398 & 2.10169 \\
\hline
\end{tabular}

\begin{tabular}{llllll} 
S & T & $M$ & \multicolumn{1}{c}{ LI } & \multicolumn{1}{c}{$W$} \\
$S$ & 11 & 4 & 1 & 857.548 & 0.0225088 \\
$S$ & 11 & 4 & 2 & 191.966 & 0.00703487 \\
$S$ & 11 & 4 & 3 & 177.168 & 0.0854821 \\
$S$ & 11 & 4 & 4 & 140.598 & 0.10644 \\
$S$ & 11 & 4 & 5 & 102.594 & 0.066616 \\
$S$ & 11 & 4 & 6 & 143.887 & 0.165463 \\
$S$ & 11 & 4 & 7 & 334.856 & 0.190439 \\
$S$ & 11 & 4 & 8 & 80.0995 & 0.100243 \\
$S$ & 11 & 4 & 9 & 162.087 & 0.314124 \\
$S$ & 11 & 4 & 10 & 58.5482 & 0.0488076 \\
$S$ & 11 & 4 & 11 & 126.257 & 0.237273 \\
$S$ & 11 & 4 & 12 & 115.51 & 0.275336 \\
$S$ & 11 & 4 & 13 & 260.035 & 0.135425 \\
$S$ & 11 & 4 & 14 & 87.9354 & 0.0235515 \\
$S$ & 11 & 4 & 15 & 128.79 & 0.111152 \\
$S$ & 11 & 4 & 16 & 89.7403 & 0.200056 \\
$S$ & 11 & 4 & 17 & 76.7992 & 0.158693 \\
$S$ & 11 & 4 & 18 & 61.5777 & 0.0590678 \\
$S$ & 11 & 4 & 19 & 85.5809 & 0.0282582 \\
$S$ & 11 & 4 & 20 & 87.6795 & 0.0875979
\end{tabular}

\section{Sample 11 Scan 5:}

\begin{tabular}{|c|c|c|c|c|}
\hline s & $\mathrm{t}$ & a & $\frac{\circ}{\circ}$ erg & $f$ \\
\hline 9.46 & 1052.24 & 0.0000644838 & 0.575799 & -0.51437 \\
\hline 55.122 & 1603.39 & $-2.30384^{\prime} 10^{-6}$ & 0.0739968 & -0.539556 \\
\hline 27.508 & 916.826 & $-9.04888^{\prime} 10^{-6}$ & 0.0381969 & 3.02942 \\
\hline 8.094 & 1100 . & $-9.53644^{\prime} 10^{-6}$ & 0.0405613 & 0.665223 \\
\hline 4.263 & 729.536 & -0.0000132051 & 0.0264054 & -2.09559 \\
\hline 8.121 & 1098.04 & -0.0000288724 & 0.0327532 & 0.638555 \\
\hline .2622 & 900. & $4.73309^{\prime} 10^{-7}$ & 0.0520551 & 0.870199 \\
\hline 57.888 & 1193.79 & $-6.8737320340321575^{\prime} 10^{-6}$ & 0.0122847 & -0.650511 \\
\hline 8.245 & 1000 & $-3.9369919032095197^{\prime} 10^{-7}$ & 0.014816 & -0.796789 \\
\hline 30.035 & 1037.62 & $1.0110043728356002^{\prime} 10^{-6}$ & 0.00753444 & -2.36476 \\
\hline 2.015 & 1200.47 & $8.21642^{\prime} 10^{-6}$ & 0.0144941 & 1.41994 \\
\hline 29.698 & 900.016 & $-1.19995^{\prime} 10^{-6}$ & 0.010485 & 0.181963 \\
\hline 33.107 & 900 . & $-1.0801015488809754^{\prime} 10^{-7}$ & 0.0123995 & 0.0870388 \\
\hline 76.509 & 903.719 & 0.0000113115 & 0.00737237 & -1.62832 \\
\hline 33.496 & 958.338 & $-9.17377^{\prime} 10^{-6}$ & 0.00969228 & 2.4465 \\
\hline 5.482 & 1393.34 & $-6.71476^{\prime} 10^{-7}$ & 0.003968 & 0.759614 \\
\hline 3.945 & 940.04 & $2.7027359092610103^{\prime} 10^{-6}$ & 0.00491936 & 2.71102 \\
\hline .956 & 1458.76 & $6.27631^{\prime} 10^{-6}$ & 0.00368035 & 1.20527 \\
\hline 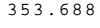 & 1445.54 & 0.0000194648 & 0.00475282 & 1.91305 \\
\hline 9.538 & 1618.48 & -0.0000177358 & 0.00446521 & -1.39696 \\
\hline
\end{tabular}

\begin{tabular}{llllll}
$S$ & $T$ & $M$ & \multicolumn{1}{c}{ LL } & \multicolumn{1}{c}{ W } \\
$S$ & 11 & 5 & 1 & 832.125 & 0.0262325 \\
$S$ & 11 & 5 & 2 & 189.779 & 0.00703124 \\
$S$ & 11 & 5 & 3 & 191.991 & 0.0757899 \\
$S$ & 11 & 5 & 4 & 227.634 & 0.102225 \\
$S$ & 11 & 5 & 5 & 259.046 & 0.16616 \\
$S$ & 11 & 5 & 6 & 308.122 & 0.190686 \\
$S$ & 11 & 5 & 7 & 105.552 & 0.237107 \\
$S$ & 11 & 5 & 8 & 73.5581 & 0.0160453 \\
$S$ & 11 & 5 & 9 & 51.5803 & 0.109111 \\
$S$ & 11 & 5 & 10 & 88.6614 & 0.206284 \\
$S$ & 11 & 5 & 11 & 131.618 & 0.31373 \\
$S$ & 11 & 5 & 12 & 171.011 & 0.191088 \\
$S$ & 11 & 5 & 13 & 148.938 & 0.162949 \\
$S$ & 11 & 5 & 14 & 103.464 & 0.0377938 \\
$S$ & 11 & 5 & 15 & 52.087 & 0.331342 \\
$S$ & 11 & 5 & 16 & 81.4743 & 0.180716 \\
$S$ & 11 & 5 & 17 & 76.2247 & 0.0878382 \\
$S$ & 11 & 5 & 18 & 36.0082 & 0.301808 \\
$S$ & 11 & 5 & 19 & 100.457 & 0.274362 \\
$S$ & 11 & 5 & 20 & 24.69 & 0.22227
\end{tabular}

\begin{tabular}{|c|c|c|c|c|}
\hline $\mathrm{s}$ & $\mathrm{t}$ & a & 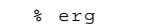 & $f$ \\
\hline 278 & 028.02 & .0000634167 & 0.564876 & 0.06780 \\
\hline 8.721 & 1597.28 & $-1.9425739240131583^{\prime} 10^{-6}$ & 0.0750616 & -0.53149 \\
\hline 9.841 & 54.54 & -0.0000307704 & 0.0626997 & 2.02061 \\
\hline 2.024 & 907.563 & -0.0000312083 & 0.0595752 & 2.44534 \\
\hline 4.72 & 1000 . & $1.2233799670839637^{\prime} 10^{-6}$ & 0.0296011 & -2.70935 \\
\hline .2898 & 901.787 & 0.000949068 & 0.0549049 & 0.16896 \\
\hline 8.483 & 1100.66 & $-5.83531 \cdot 10^{-6}$ & 0.0152406 & 0.300076 \\
\hline 6.817 & 1172.61 & 0.000116791 & 0.00970909 & -1.53181 \\
\hline 33.068 & 1143.51 & $-2.2586243034754917^{\prime} 10^{-6}$ & 0.00639246 & 1.57783 \\
\hline 0.638 & 1100 & $-7.3966^{\prime} 1$ & 0.00618964 & -2.51432 \\
\hline 1.888 & 1000 . & 0 . & .009 & -0.824979 \\
\hline 8.635 & 1505.13 & 0.0000314829 & 0.0100059 & -2.51198 \\
\hline 3.127 & 1000 . & $3.1129731680475602^{\prime} 10^{-6}$ & 0.00704428 & -0.273772 \\
\hline 9.322 & 911.152 & -0.0000206584 & 0.00863202 & -2.84815 \\
\hline 0.185 & 900.282 & $-3.1362218793415093^{\prime} 10^{-6}$ & 0.00400681 & 1.53895 \\
\hline 5.322 & 1129.75 & 0.0000166388 & 0.00588171 & 1.81033 \\
\hline 2.485 & 1604.55 & -0.0000149294 & 0.00508533 & -0.218643 \\
\hline 2.435 & 995.069 & $-7.42294^{\prime} 10^{-7}$ & 0.00227077 & 0.620605 \\
\hline 6.935 & 999.926 & $7.81743^{\prime} 10^{-6}$ & 0.00471674 & -2.12505 \\
\hline 3.467 & 878.801 & $-1.6897662722253736^{\prime} 10^{-6}$ & 0.00139539 & -2.61889 \\
\hline
\end{tabular}




\section{Sample 11 Scan 6:}

\begin{tabular}{|c|c|c|c|c|c|c|c|c|c|c|}
\hline $\mathrm{s}$ & s & $\mathrm{T}$ & M & LL & w & s & $\mathrm{t}$ & a & 음 erg & $f$ \\
\hline $\mathrm{s}$ & 11 & 6 & 1 & 825.553 & 0.0262859 & 225.854 & 1027.63 & 0.0000631776 & 0.562007 & 0.0856923 \\
\hline S & 11 & 6 & 2 & 183.58 & 0.00701766 & 520.767 & 1596.19 & $-1.98572^{\prime} 10^{-6}$ & 0.0733131 & -0.509702 \\
\hline $\mathrm{s}$ & 11 & 6 & 3 & 170.922 & 0.084257 & 240.024 & 968.064 & -0.0000163341 & 0.0446004 & -1.01229 \\
\hline $\mathrm{s}$ & 11 & 6 & 4 & 322.149 & 0.103223 & 232.167 & 934.96 & -0.0000249099 & 0.0607171 & -0.496633 \\
\hline $\mathrm{s}$ & 11 & 6 & 5 & 140.21 & 0.165523 & 429.623 & 1099.81 & -0.0000288168 & 0.0337865 & 0.399541 \\
\hline $\mathrm{s}$ & 11 & 6 & 6 & 99.2508 & 0.0663244 & 457.342 & 754.892 & -0.0000136346 & 0.021209 & 2.63953 \\
\hline $\mathrm{s}$ & 11 & 6 & 7 & 517.975 & 0.190595 & 44.3408 & 900.108 & $-6.27637^{\prime} 10^{-7}$ & 0.060079 & 0.903113 \\
\hline $\mathrm{s}$ & 11 & 6 & 8 & 69.9869 & 0.0260015 & 134.018 & 1139.4 & 0.000115118 & 0.00967832 & -0.82671 \\
\hline $\mathrm{s}$ & 11 & 6 & 9 & 137.886 & 0.237275 & 84.8364 & 1199.64 & $1.1987040767150602^{\prime} 10^{-6}$ & 0.0166055 & 1.76094 \\
\hline $\mathrm{S}$ & 11 & 6 & 10 & 166.148 & 0.313957 & 266.52 & 1000.01 & $-7.40331^{\prime} 10^{-7}$ & 0.0117708 & -0.765072 \\
\hline s & 11 & 6 & 11 & 207.647 & 0.13563 & 229.45 & 899.974 & $5.07426^{\prime} 10^{-6}$ & 0.0104561 & -0.0857003 \\
\hline $\mathrm{s}$ & 11 & 6 & 12 & 109.769 & 0.274543 & 263.649 & 999.969 & 0 . & 0.00789862 & -2.08979 \\
\hline $\mathrm{s}$ & 11 & 6 & 13 & 101.197 & 0.0364366 & 634.414 & 899.808 & -0.0000245616 & 0.00835089 & -2.06275 \\
\hline s & 11 & 6 & 14 & 92.5783 & 0.192657 & 741.493 & 1345.72 & -0.0000279622 & 0.00693297 & -2.16118 \\
\hline $\mathrm{s}$ & 11 & 6 & 15 & 50.7804 & 0.0445518 & 723.67 & 1228.92 & 0.0000250747 & 0.00353889 & -1.49556 \\
\hline S & 11 & 6 & 16 & 47.3887 & 0.331321 & 278.576 & 961.903 & $2.50828^{\prime} 10^{-6}$ & 0.00314291 & -0.144306 \\
\hline $\mathrm{s}$ & 11 & 6 & 17 & 76.7568 & 0.102691 & 289.134 & 1643.02 & 0.0000287967 & 0.00478694 & 3.02624 \\
\hline s & 11 & 6 & 18 & 80.3958 & 0.162118 & 241.772 & 1119.99 & -0.0000138111 & 0.00462547 & -0.16029 \\
\hline s & 11 & 6 & 19 & 45.382 & 0.303144 & 866.88 & 1071.51 & $-8.4884^{\prime} 10^{-6}$ & 0.00245693 & -2.83629 \\
\hline S & 11 & 6 & 20 & 67.0102 & 0.22928 & 317.769 & 857.437 & $-8.43238^{\prime} 10^{-6}$ & 0.00342995 & 1.12619 \\
\hline
\end{tabular}

\section{Sample 11 Scan 7:}

\begin{tabular}{lllllll}
$\mathrm{S}$ & $\mathrm{T}$ & $\mathrm{M}$ & \multicolumn{1}{c}{$\mathrm{LL}$} & \multicolumn{1}{c}{$\mathrm{W}$} & \multicolumn{1}{c}{$\mathrm{S}$} \\
$\mathrm{S}$ & 11 & 7 & 1 & 823.48 & 0.0262973 & 225.944 \\
$\mathrm{~S}$ & 11 & 7 & 2 & 181.387 & 0.00700816 & 518.653 \\
$\mathrm{~S}$ & 11 & 7 & 3 & 251.933 & 0.0884815 & 78.0538 \\
$\mathrm{~S}$ & 11 & 7 & 4 & 157.194 & 0.166253 & 250.79 \\
$\mathrm{~S}$ & 11 & 7 & 5 & 230.147 & 0.190663 & 36.1583 \\
$\mathrm{~S}$ & 11 & 7 & 6 & 140.052 & 0.0991884 & 251.392 \\
$\mathrm{~S}$ & 11 & 7 & 7 & 100.941 & 0.0519975 & 439.006 \\
$\mathrm{~S}$ & 11 & 7 & 8 & 107.661 & 0.112625 & 244.026 \\
$\mathrm{~S}$ & 11 & 7 & 9 & 104.785 & 0.236857 & 253.877 \\
$\mathrm{~S}$ & 11 & 7 & 10 & 47.9144 & 0.0883501 & 822.006 \\
$\mathrm{~S}$ & 11 & 7 & 11 & 166.907 & 0.314225 & 207.055 \\
$\mathrm{~S}$ & 11 & 7 & 12 & 139.912 & 0.162968 & -56.1731 \\
$\mathrm{~S}$ & 11 & 7 & 13 & 85.628 & 0.0229584 & 540.637 \\
$\mathrm{~S}$ & 11 & 7 & 14 & 130.172 & 0.183997 & 125.573 \\
$\mathrm{~S}$ & 11 & 7 & 15 & 79.8163 & 0.055712 & 131.303 \\
$\mathrm{~S}$ & 11 & 7 & 16 & 118.568 & 0.27409 & 202.318 \\
$\mathrm{~S}$ & 11 & 7 & 17 & 67.439 & 0.204028 & 102.379 \\
$\mathrm{~S}$ & 11 & 7 & 18 & 82.8563 & 0.329555 & 212.111 \\
$\mathrm{~S}$ & 11 & 7 & 19 & 63.7844 & 0.106304 & 199.123 \\
$\mathrm{~S}$ & 11 & 7 & 20 & 34.5581 & 0.163037 & 1093.51
\end{tabular}

\begin{tabular}{|c|c|c|c|}
\hline $\mathrm{t}$ & a & $\frac{\circ}{\partial}$ erg & $f$ \\
\hline 1027.11 & 0.0000633181 & 0.561098 & 0.0852068 \\
\hline 1595.36 & $-1.93627^{\prime} 10^{-6}$ & 0.0726678 & -0.512008 \\
\hline 845.536 & 0.0000774016 & 0.081316 & 2.31116 \\
\hline 1000 . & $6.31873^{\prime} 10^{-7}$ & 0.0291543 & -2.72906 \\
\hline 904.28 & 0.000385876 & 0.052354 & -0.223922 \\
\hline 1000 . & $-2.80688^{\prime} 10^{-7}$ & 0.0216343 & -2.06214 \\
\hline 766.728 & 0.0000228482 & 0.017347 & 3.02726 \\
\hline 1000 . & $5.72335^{\prime} 10^{-8}$ & 0.0133585 & -1.022 \\
\hline 1102.07 & $-4.61369^{\prime} 10^{-6}$ & 0.0149038 & -0.0753922 \\
\hline 1411.36 & $-1.2926573350193622^{\prime} 10^{-6}$ & 0.00631889 & -2.40101 \\
\hline 1000 . & $-1.1996502471492275^{\prime} 10^{-6}$ & 0.0133735 & -0.77728 \\
\hline 1000 . & $2.62075^{\prime} 10^{-7}$ & 0.0122675 & -0.503116 \\
\hline 966.721 & $8.0923^{\prime} 10^{-6}$ & 0.00846109 & 3.09291 \\
\hline 1499.37 & 0 . & 0.0104079 & -1.23292 \\
\hline 1390.45 & 0.0000947061 & 0.00646193 & -1.36103 \\
\hline 1000 & $5.07464^{\prime} 10^{-6}$ & 0.00686591 & -2.15464 \\
\hline 1199.63 & $-4.00582^{\prime} 10^{-6}$ & 0.00459788 & 1.6465 \\
\hline 947.778 & -0.0000530114 & 0.00372488 & -0.863389 \\
\hline 1580.43 & $-5.224^{\prime} 10^{-7}$ & 0.00386716 & -2.60481 \\
\hline 1757.11 & $-4.97339^{\prime} 10^{-7}$ & 0.00199444 & 2.8474 \\
\hline
\end{tabular}

\section{Sample 11 Scan 8:}

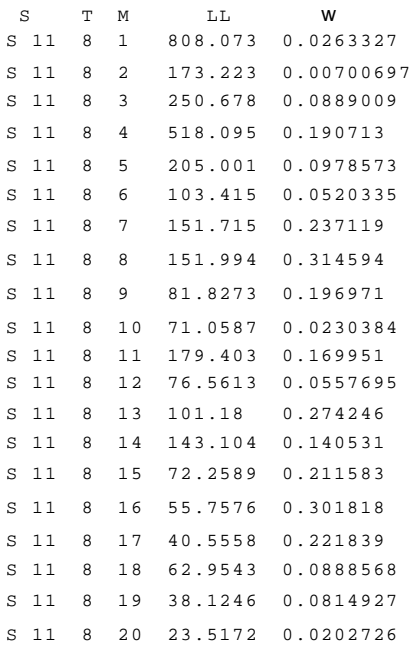

\begin{tabular}{|c|c|c|c|c|}
\hline $\mathrm{s}$ & $\mathrm{t}$ & a & $\therefore$ erg & $f$ \\
\hline 25.83 & 1026.63 & 0.000063338 & 0.554284 & 0.0905156 \\
\hline 515.28 & 1592.96 & $-1.9515736297086244^{\prime} 10^{-6}$ & 0.0707582 & -0.493563 \\
\hline 77.2228 & 845.355 & 0.0000777647 & 0.0828937 & 2.31226 \\
\hline 46.6654 & 900.003 & $-6.81865^{\prime} 10^{-7}$ & 0.083432 & 0.859653 \\
\hline 06.657 & 1100.01 & $3.1396545203695316^{\prime} 10^{-6}$ & 0.0313338 & 0.399781 \\
\hline 40.404 & 765.514 & 0.0000226229 & 0.0173108 & 3.0938 \\
\hline 225.515 & 1100 & $3.1457446022357064^{\prime} 10^{-6}$ & 0.0140907 & 0.370361 \\
\hline 167.317 & 1100 & $-2.3700124261865146^{\prime} 10^{-8}$ & 0.012874 & -0.671856 \\
\hline 10.749 & 1500.01 & $1.1978661077624558^{\prime} 10^{-6}$ & 0.0103055 & -1.49483 \\
\hline 38.412 & 962.112 & $8.07872^{\prime} 10^{-6}$ & 0.00834189 & -3.08223 \\
\hline 03.582 & 1100. & -0.0000145675 & 0.0115488 & 0.127087 \\
\hline 2.905 & 1397.48 & -0.0000862239 & 0.00749744 & -1.64857 \\
\hline 262.225 & 969.818 & $5.09053^{\prime} 10^{-6}$ & 0.00900511 & -0.0988759 \\
\hline 171.372 & 900. & 0.0000356941 & 0.00963557 & -0.189205 \\
\hline 179.845 & 1213.42 & $-9.98502^{\prime} 10^{-7}$ & 0.00489788 & -1.29194 \\
\hline 508.901 & 984.379 & $-7.59767^{\prime} 10^{-6}$ & 0.00383339 & -1.46652 \\
\hline 1377.95 & 1586.93 & $2.41559^{\prime} 10^{-6}$ & 0.002657 & 1.62018 \\
\hline 951.011 & 1947.96 & 0.0000170989 & 0.00391678 & -0.498719 \\
\hline 1409.34 & 1227.68 & $-1.3950953486762136^{\prime} 10^{-} 6$ & 0.00225481 & -0.969864 \\
\hline 59.875 & 1644.17 & $7.17534^{\prime} 10^{-6}$ & 0.00134861 & -2.97859 \\
\hline
\end{tabular}




\section{Sample 11 Scan 9:}

\begin{tabular}{|c|c|c|c|c|c|c|c|c|c|c|}
\hline$s$ & $\mathrm{~s}$ & $\mathrm{~T}$ & 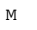 & LL & w & 5 & $\mathrm{t}$ & a & 음 erg & $f$ \\
\hline $\mathrm{s}$ & 11 & 9 & 1 & 809.099 & .026343 & 225.461 & 026.4 & 0.000063399 & 0.554741 & 0.09087 \\
\hline $\mathrm{S}$ & 11 & 9 & 2 & 173.383 & 0.00699692 & 516.876 & 1592.5 & $-1.92825^{\prime} 10^{-6}$ & 0.0707473 & -0.494475 \\
\hline S & 11 & 9 & 3 & 255.413 & 0.0888551 & 77.6111 & 845.329 & 0.000078506 & 0.0841719 & 2.31361 \\
\hline $\mathrm{s}$ & 11 & 9 & 4 & 373.645 & 0.190708 & 42.917 & 900.133 & $-1.1590876028305054^{\prime} 10^{-6}$ & 0.0843473 & 0.841932 \\
\hline S & 11 & 9 & 5 & 205.353 & 0.0977672 & 109.159 & 1100. & $2.6920102957270044^{\prime} 10^{-7}$ & 0.0302037 & 0.410591 \\
\hline S & 11 & 9 & 6 & 105.77 & 0.0521985 & 39.042 & 762.285 & 0.0000227331 & 0.017535 & -3.03009 \\
\hline S & 11 & 9 & 7 & 110.704 & 0.237457 & 238.792 & 1100. & $2.4955618066215718^{\prime} 10^{-8}$ & 0.0156536 & 0.405265 \\
\hline $\mathrm{s}$ & 11 & 9 & 8 & 145.015 & 0.31483 & 72.382 & 1100 . & $1.17489^{\prime} 10^{-6}$ & 0.01198 & -0.690169 \\
\hline $\mathrm{s}$ & 11 & 9 & 9 & 66.9233 & 0.0230827 & 535.186 & 960.357 & $8.08797^{\prime} 10^{-6}$ & 0.00838532 & -3.04576 \\
\hline S & 11 & 9 & 10 & 93.5785 & 0.196707 & 119.448 & 1500 . & $-3.6963225664015914^{\prime} 10^{-6}$ & 0.0107512 & -1.48903 \\
\hline & 11 & 9 & 11 & 160.48 & 0.170005 & 8.397 & 1100. & -0.0000166615 & 0.011874 & 0.156099 \\
\hline S & 11 & 9 & 12 & 79.2287 & .0557404 & 4.7225 & 397.41 & -0.0000414884 & 0.00750488 & -1.65742 \\
\hline S & 11 & 9 & 13 & 90.1899 & 0.273625 & 61.274 & 1000 . & 0 & 0.00760233 & -1.90301 \\
\hline $\mathrm{S}$ & 11 & 9 & 14 & 200.511 & 0.127397 & 70.6195 & 984.061 & 0.0000298893 & 0.0111834 & 0.806156 \\
\hline $\mathrm{s}$ & 11 & 9 & 15 & 85.7776 & 0.212141 & 9.2 & 1100. & $-4.49774^{\prime} 10^{-8}$ & 0.00423131 & -2.26093 \\
\hline $\mathrm{S}$ & 11 & 9 & 16 & 89.1332 & 0.101958 & 85.0007 & 1674.71 & -0.000141678 & 0.00579897 & 0.123037 \\
\hline $\mathrm{s}$ & 11 & 9 & 17 & 66.7847 & 0.301972 & 420.161 & 1006.69 & $-5.80364^{\prime} 10^{-6}$ & 0.00401854 & -2.03152 \\
\hline $\mathrm{s}$ & 11 & 9 & 18 & 25.0179 & 0.0202384 & 72.598 & 1643.87 & $7.1191041913165775^{\prime} 10^{-6}$ & 0.0014376 & -2.99059 \\
\hline 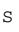 & 11 & s & 19 & 34.3943 & 0.221173 & 2469.44 & 2294.72 & $1.0906186913153365^{\prime} 10^{-6}$ & 0.00191858 & . \\
\hline & & & & & & & & 9.65912 & 86232 & .448 \\
\hline
\end{tabular}

\section{Sample 11 Scan 10:}

\begin{tabular}{llllll} 
S & T & $M$ & \multicolumn{1}{c}{ LL } & \multicolumn{1}{c}{ W } \\
S & 11 & 10 & 1 & 803.287 & 0.0263537 \\
$\mathrm{~S}$ & 11 & 10 & 2 & 171.25 & 0.00700129 \\
$\mathrm{~S}$ & 11 & 10 & 3 & 251.095 & 0.0888304 \\
$\mathrm{~S}$ & 11 & 10 & 4 & 363.176 & 0.190873 \\
$\mathrm{~S}$ & 11 & 10 & 5 & 90.1729 & 0.0523995 \\
$\mathrm{~S}$ & 11 & 10 & 6 & 133.609 & 0.0986875 \\
$\mathrm{~S}$ & 11 & 10 & 7 & 125.037 & 0.237989 \\
$\mathrm{~S}$ & 11 & 10 & 8 & 64.4637 & 0.0874983 \\
$\mathrm{~S}$ & 11 & 10 & 9 & 86.4822 & 0.113248 \\
$\mathrm{~S}$ & 11 & 10 & 10 & 96.3192 & 0.196354 \\
$\mathrm{~S}$ & 11 & 10 & 11 & 178.353 & 0.314423 \\
$\mathrm{~S}$ & 11 & 10 & 12 & 205.209 & 0.169767 \\
$\mathrm{~S}$ & 11 & 10 & 13 & 95.7363 & 0.0231445 \\
$\mathrm{~S}$ & 11 & 10 & 14 & 82.8889 & 0.0555773 \\
$\mathrm{~S}$ & 11 & 10 & 15 & 49.964 & 0.302332 \\
$\mathrm{~S}$ & 11 & 10 & 16 & 97.3147 & 0.223932 \\
$\mathrm{~S}$ & 11 & 10 & 17 & 93.7313 & 0.274188 \\
$\mathrm{~S}$ & 11 & 10 & 18 & 56.9325 & 0.329847 \\
$\mathrm{~S}$ & 11 & 10 & 19 & 80.5574 & 0.105112 \\
$\mathrm{~S}$ & 11 & 10 & 20 & 46.2312 & 0.0480535
\end{tabular}

Sample 11 Scan 11:

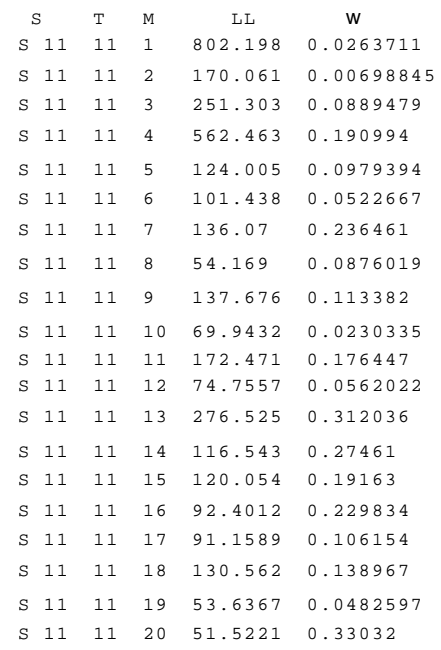

\begin{tabular}{|c|c|c|c|c|}
\hline $\mathrm{s}$ & $t$ & a & $\div$ erg & $f$ \\
\hline 25.366 & 1026.39 & 0.0000633828 & 0.552146 & 0.0926143 \\
\hline 6.9 & 1591.09 & $-1.9404314456317194^{\prime} 10^{-6}$ & 0.0703579 & -0.484385 \\
\hline .1907 & 845.186 & 0.0000843875 & 0.0835854 & 2.32029 \\
\hline .5386 & 902.057 & 0.000266063 & 0.0891794 & 0.356012 \\
\hline 4.335 & 759.319 & 0.0000231113 & 0.0175609 & -2.90118 \\
\hline 0.925 & 1000 . & $5.99718^{\prime} 10^{-8}$ & 0.0200079 & -2.10523 \\
\hline 2.279 & 1086.54 & -0.0000132262 & 0.0195215 & -2.44388 \\
\hline 7.092 & 1282.77 & $-4.96418^{\prime} 10^{-7}$ & 0.00915025 & 2.34115 \\
\hline 54.686 & 1000 . & 0 . & 0.0112434 & -0.820101 \\
\hline .7 .572 & 1499.49 & $-4.08076^{\prime} 10^{-6}$ & 0.0112497 & -1.39176 \\
\hline 9.653 & 1100 . & $1.50471^{\prime} 10^{-7}$ & 0.0110839 & -0.585127 \\
\hline 2.506 & 1099.79 & -0.0000205898 & 0.0123165 & 0.324582 \\
\hline 2.991 & 956.851 & $8.22602^{\prime} 10^{-6}$ & 0.00835586 & -2.9727 \\
\hline 5.124 & 1392.29 & 0.0000999687 & 0.00661578 & -1.48678 \\
\hline 7.196 & 1021.15 & $-7.14043^{\prime} 10^{-6}$ & 0.00373086 & -0.271912 \\
\hline .1799 & 1200.39 & $1.8538524183630554^{\prime} 10^{-6}$ & 0.00618573 & 1.26306 \\
\hline 1.955 & 1020.36 & 0.0000331567 & 0.00594993 & -1.74599 \\
\hline 6.211 & 1041.79 & $6.5236^{\prime} 10^{-6}$ & 0.00335846 & -1.53035 \\
\hline 2.795 & 1612.82 & $-9.30371^{\prime} 10^{-6}$ & 0.00443699 & 0.319244 \\
\hline .316 & 43.288 & $-5.41362^{\prime} 10^{-6}$ & 0.0023895 & 1.09799 \\
\hline
\end{tabular}

\begin{tabular}{|c|c|c|c|c|}
\hline $\mathrm{s}$ & $\mathrm{t}$ & a & $\div$ erg & f \\
\hline 224.977 & 1026.38 & 0.0000632419 & 0.551658 & 0.100409 \\
\hline 19.068 & 1590.6 & $-1.97274^{\prime} 10^{-6}$ & 0.0699883 & -0.47067 \\
\hline 6.8322 & 844.999 & 0.0000847513 & 0.0838406 & 2.34554 \\
\hline 44.1628 & 899.995 & $-1.9596436673988834^{\prime} 10^{-6}$ & 0.090041 & 0.880817 \\
\hline 89.453 & 1000 . & $-1.0885248195933514^{\prime} 10^{-7}$ & 0.0180123 & -2.1856 \\
\hline 37.645 & 760.976 & 0.000022811 & 0.0178846 & -2.95762 \\
\hline 70.4082 & 1199.76 & $1.1509889484576553^{\prime} 10^{-6}$ & 0.020671 & 1.74849 \\
\hline 816.121 & 1322.77 & $-8.36766^{\prime} 10^{-7}$ & 0.00774302 & -1.03002 \\
\hline 46.415 & 1000 . & $-1.2187215398819232^{\prime} 10^{-9}$ & 0.0141635 & -0.80976 \\
\hline 35.808 & 961.533 & $8.05494^{\prime} 10^{-6}$ & 0.00844021 & -3.06147 \\
\hline 40.959 & 1290.64 & -0.0000290145 & 0.0184558 & -0.803185 \\
\hline 47.523 & 1390.48 & 0.000107936 & 0.00706219 & -1.4177 \\
\hline 118.901 & 1000 & $5.44772^{\prime} 10^{-7}$ & 0.0129804 & -0.485641 \\
\hline 244.285 & 1000 & $-1.9382053890030102^{\prime} 10^{-6}$ & 0.00725517 & -2.09724 \\
\hline 675.5 & 1322.35 & -0.0000277421 & 0.00800316 & 2.4161 \\
\hline 34.214 & 867.984 & $-8.12384^{\prime} 10^{-6}$ & 0.00553763 & -1.33778 \\
\hline 235.602 & 1565.28 & 0.0000145666 & 0.00498404 & -1.10796 \\
\hline 209.642 & 900 . & $-1.93821 \cdot 10^{-6}$ & 0.00520705 & 0.0982366 \\
\hline 553.02 & 759.564 & $-5.74513^{\prime} 10^{-6}$ & 0.00245519 & 0.343459 \\
\hline 42.74 & 900. & -0.0000322741 & 0.00216155 & 2.53164 \\
\hline
\end{tabular}


Sample 11 Scan 12:

\begin{tabular}{lllllll}
$S$ & $S$ & $M$ & \multicolumn{1}{c}{ LL } & \multicolumn{1}{c}{ W } & \multicolumn{1}{c}{ S } \\
S & 11 & 12 & 1 & 823.318 & 0.0264219 & 224.695 \\
$\mathrm{~S}$ & 11 & 12 & 2 & 176.532 & 0.0069991 & 516.521 \\
$\mathrm{~S}$ & 11 & 12 & 3 & 270.343 & 0.0892416 & 76.0961 \\
$\mathrm{~S}$ & 11 & 12 & 4 & 539.585 & 0.190889 & 51.4627 \\
$\mathrm{~S}$ & 11 & 12 & 5 & 126.585 & 0.0978796 & 222.503 \\
$\mathrm{~S}$ & 11 & 12 & 6 & 93.3028 & 0.0598815 & 186.596 \\
$\mathrm{~S}$ & 11 & 12 & 7 & 47.3151 & 0.0877495 & 848.409 \\
$\mathrm{~S}$ & 11 & 12 & 8 & 100.709 & 0.112544 & 517.438 \\
$\mathrm{~S}$ & 11 & 12 & 9 & 67.2329 & 0.0286074 & 98.2495 \\
$\mathrm{~S}$ & 11 & 12 & 10 & 155.948 & 0.236163 & 56.9163 \\
$\mathrm{~S}$ & 11 & 12 & 11 & 143.87 & 0.191968 & 132.55 \\
$\mathrm{~S}$ & 11 & 12 & 12 & 111.416 & 0.168349 & 160.368 \\
$\mathrm{~S}$ & 11 & 12 & 13 & 105.423 & 0.274973 & 224.729 \\
$\mathrm{~S}$ & 11 & 12 & 14 & 150.441 & 0.143002 & 109.989 \\
$\mathrm{~S}$ & 11 & 12 & 15 & 128.737 & 0.0306057 & 250.24 \\
$\mathrm{~S}$ & 11 & 12 & 16 & 308.785 & 0.312404 & -96.2621 \\
$\mathrm{~S}$ & 11 & 12 & 17 & 156.891 & 0.060756 & 149.924 \\
$\mathrm{~S}$ & 11 & 12 & 18 & 102.129 & 0.230762 & 401.908 \\
$\mathrm{~S}$ & 11 & 12 & 19 & 56.6559 & 0.104686 & 337.937 \\
$\mathrm{~S}$ & 11 & 12 & 20 & 55.1913 & 0.206259 & 192.526
\end{tabular}

\begin{tabular}{|c|c|c|c|}
\hline$t$ & a & $\frac{\circ}{b}$ erg & $f$ \\
\hline 1026.45 & 0.0000631206 & 0.561027 & 0.111109 \\
\hline 1589.98 & $-2.0045565469870263^{\prime} 10^{-6}$ & 0.0709036 & -0.459181 \\
\hline 844.813 & 0.0000797498 & 0.0869307 & 2.40726 \\
\hline 900.306 & $8.09912^{\prime} 10^{-6}$ & 0.0884631 & 0.926077 \\
\hline 999.996 & $-1.1978812205940977^{\prime} 10^{-6}$ & 0.0179088 & -2.17625 \\
\hline 587.428 & $2.69643^{\prime} 10^{-6}$ & 0.0154714 & 0.820078 \\
\hline 1334.05 & $-5.63527^{\prime} 10^{-7}$ & 0.00731112 & -1.99409 \\
\hline 1162.98 & $5.87646^{\prime} 10^{-6}$ & 0.0144561 & -0.516978 \\
\hline 1140.72 & 0.0000410021 & 0.00887146 & -0.681402 \\
\hline 1199.75 & $-1.8631907318882845^{\prime} 10^{-6}$ & 0.0176156 & 1.78593 \\
\hline 1506.76 & -0.0000382132 & 0.0143181 & -2.46312 \\
\hline 1100 . & $-3.82921^{\prime} 10^{-7}$ & 0.00828986 & 0.232159 \\
\hline 1000.31 & $1.2039005687226186^{\prime} 10^{-6}$ & 0.00727072 & -2.03683 \\
\hline 999.996 & $8.64672^{\prime} 10^{-8}$ & 0.00961824 & -1.1557 \\
\hline 725.09 & -0.0000305699 & 0.00850906 & -2.7448 \\
\hline 1000 . & $-1.9396335055704004^{\prime} 10^{-6}$ & 0.0112165 & -0.364616 \\
\hline 1387.72 & 0.000209864 & 0.00736361 & -1.55962 \\
\hline 921.956 & $-7.31117^{\prime} 10^{-6}$ & 0.00420737 & -1.22689 \\
\hline 733.431 & $-3.30546^{\prime} 10^{-6}$ & 0.00215524 & 2.39886 \\
\hline 719.857 & -0.000025058 & 0.00197202 & -3.10439 \\
\hline
\end{tabular}

\section{Sample 11 Scan 13:}

\begin{tabular}{llllll}
$S$ & $T$ & $M$ & \multicolumn{1}{c}{ LL } & \multicolumn{1}{c}{ W } \\
$S$ & 11 & 13 & 1 & 1162.9 & 0.022124 \\
$S$ & 11 & 13 & 2 & 329.763 & 0.00698984 \\
$S$ & 11 & 13 & 3 & 352.103 & 0.0848904 \\
$S$ & 11 & 13 & 4 & 100.791 & 0.0594063 \\
$S$ & 11 & 13 & 5 & 393.03 & 0.19097 \\
$S$ & 11 & 13 & 6 & 127.783 & 0.0985586 \\
$S$ & 11 & 13 & 7 & 64.0219 & 0.0877513 \\
$S$ & 11 & 13 & 8 & 108.888 & 0.0229714 \\
$S$ & 11 & 13 & 9 & 117.871 & 0.0476548 \\
$S$ & 11 & 13 & 10 & 130.409 & 0.112539 \\
$S$ & 11 & 13 & 11 & 156.75 & 0.237245 \\
$S$ & 11 & 13 & 12 & 126.992 & 0.194843 \\
$S$ & 11 & 13 & 13 & 111.714 & 0.0266233 \\
$S$ & 11 & 13 & 14 & 166.261 & 0.169115 \\
$S$ & 11 & 13 & 15 & 58.5304 & 0.216629 \\
$S$ & 11 & 13 & 16 & 143.776 & 0.143215 \\
$S$ & 11 & 13 & 17 & 45.139 & 0.0399671 \\
$S$ & 11 & 13 & 18 & 203.914 & 0.31567 \\
$S$ & 11 & 13 & 19 & 68.2227 & 0.102096 \\
$S$ & 11 & 13 & 20 & 151.268 & 0.272585
\end{tabular}

\begin{tabular}{|c|c|c|c|c|}
\hline s & $\mathrm{t}$ & a & $\%$ erg & . \\
\hline 39.312 & 1060.86 & 0.0000632794 & 0.687421 & -0.55145 \\
\hline 554.215 & 1596.68 & $-2.5657161553944113^{\prime} 10^{-6}$ & 0.0875983 & -0.411815 \\
\hline 81.9485 & 853.266 & 0.000123813 & 0.0665152 & 1.97258 \\
\hline 93.84 & 589.426 & $-3.1760389600945336^{\prime} 10^{-6}$ & 0.0151103 & 1.04847 \\
\hline 5.8547 & 900. & $-6.99349^{\prime} 10^{-7}$ & 0.0419278 & 1.24149 \\
\hline 208.893 & 1000.08 & $-7.3372^{\prime} 10^{-7}$ & 0.0118338 & -1.71054 \\
\hline 333.486 & 1340.39 & $-6.29527^{\prime} 10^{-7}$ & 0.00550242 & -2.1773 \\
\hline 48.116 & 902.099 & $8.04642^{\prime} 10^{-6}$ & 0.00858616 & -1.26035 \\
\hline 526.679 & 1162.39 & -0.0000193018 & 0.00829892 & -1.0907 \\
\hline 55.255 & 1157.22 & $5.98665^{\prime} 10^{-6}$ & 0.00811087 & 0.486045 \\
\hline 239.754 & 1099.99 & $-6.1579^{\prime} 10^{-6}$ & 0.00615248 & 0.903064 \\
\hline .47 .04 & 1510.49 & -0.0000453781 & 0.0062103 & -2.90244 \\
\hline 250.109 & 1508.45 & 0.0000174701 & 0.00484798 & 0.504283 \\
\hline 39.307 & 1100 & $-1.4925316108882747^{\prime} 1$ & 0.0036739 & 0.548957 \\
\hline 270.364 & 1063.82 & -0.000014909 & 0.00212235 & 0.0340753 \\
\hline 249.94 & 1000.01 & $-1.93821 \cdot 10^{-6}$ & 0.00368458 & -1.01273 \\
\hline 3767.65 & 7708.35 & $3.202^{\prime} 10^{-7}$ & 0.00139188 & -2.32563 \\
\hline 238.203 & 1100 & $-2.1822039622323164^{\prime} 10^{-6}$ & 0.0030812 & -0.457645 \\
\hline 54.254 & 1224.01 & $3.99093^{\prime} 10^{-6}$ & 0.00178476 & 1.79273 \\
\hline 16.973 & 1000.31 & 0.0000279733 & 0.00301999 & -2.00251 \\
\hline
\end{tabular}

\section{Sample 11 Scan 14:}$$
240.0
$$

240.077

555.969

83.777

191.496

$$
49.0295
$$

783.051

197.692

569.401

792.539

469.845

221.426

$-60.3612$

143.353

951.282

141.958

160.405

197.383

280.729

151.765

186.775

\begin{tabular}{|c|c|c|c|}
\hline$t$ & a & $\frac{\circ}{b}$ erg & $f$ \\
\hline 1070.59 & 0.0000626107 & 0.730976 & -0.670826 \\
\hline 1597.02 & $-2.6894842777907805^{\prime} 10^{-6}$ & 0.0969416 & -0.383696 \\
\hline 850.624 & 0.000123024 & 0.0464614 & 2.34226 \\
\hline 589.523 & $-6.3709329126843495^{\prime} 10^{-6}$ & 0.0133212 & 1.23575 \\
\hline 900 . & $5.8428^{\prime} 10^{-7}$ & 0.0323142 & 1.2204 \\
\hline 728.686 & -0.0000250233 & 0.0108035 & -2.6544 \\
\hline 1000.05 & $-4.98786^{\prime} 10^{-6}$ & 0.00796522 & -1.61735 \\
\hline 1403.72 & $-6.2092165129321855^{\prime} 10^{-6}$ & 0.00626652 & 2.57537 \\
\hline 1291.07 & $-6.82384^{\prime} 10^{-7}$ & 0.00369491 & 2.25626 \\
\hline 1278.21 & -0.0000145907 & 0.00450088 & 1.89477 \\
\hline 1000. & $1.2391384704017949^{\prime} 10^{-6}$ & 0.00397244 & -0.418899 \\
\hline 1200. & $8.35349^{\prime} 10^{-7}$ & 0.00602987 & 1.99702 \\
\hline 1499.28 & -0.000037825 & 0.00493629 & -0.746211 \\
\hline 1247.46 & -0.0000125744 & 0.00259883 & 0.444624 \\
\hline 1099.92 & $-1.9327441960126343^{\prime} 10^{-6}$ & 0.00259718 & 0.540287 \\
\hline 1099.79 & $-1.938210^{\prime}-6$ & 0.00286918 & -0.354725 \\
\hline 999.989 & $2.9638470333872844^{\prime} 10^{-6}$ & 0.00231914 & -1.04243 \\
\hline 1000.31 & $1.1979301616470875^{\prime} 10^{-6}$ & 0.0017318 & -1.87477 \\
\hline 00 . & $-2.75944^{\prime} 10^{-6}$ & 0.00144387 & -0.0908747 \\
\hline 95.09 & $9.90639^{\prime} 10^{-6}$ & 0.00202586 & 2.59325 \\
\hline
\end{tabular}




\section{Sample 11 Scan 15:}

\begin{tabular}{|c|c|c|c|c|c|}
\hline 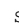 & & $\mathrm{T}$ & M & LL & w \\
\hline $\mathrm{s}$ & 11 & 15 & 1 & 1226.29 & 0.0219245 \\
\hline $\mathrm{s}$ & 11 & 15 & 2 & 371.785 & 0.00699984 \\
\hline s & 11 & 15 & 3 & 344.892 & 0.083856 \\
\hline $\mathrm{s}$ & 11 & 15 & 4 & 111.281 & 0.0594395 \\
\hline $\mathrm{s}$ & 11 & 15 & 5 & 409.745 & 0.190438 \\
\hline s & 11 & 15 & 6 & 126.909 & 0.0985843 \\
\hline s & 11 & 15 & 7 & 78.2475 & 0.0876376 \\
\hline $\mathrm{s}$ & 11 & 15 & 8 & 130.061 & 0.0229207 \\
\hline $\mathrm{s}$ & 11 & 15 & 9 & 134.548 & 0.047315 \\
\hline $\mathrm{S}$ & 11 & 15 & 10 & 166.353 & 0.236665 \\
\hline $\mathrm{s}$ & 11 & 15 & 11 & 123.939 & 0.113655 \\
\hline s & 11 & 15 & 12 & 126.461 & 0.025413 \\
\hline S & 11 & 15 & 13 & 237.519 & 0.192782 \\
\hline s & 11 & 15 & 14 & 46.0668 & 0.0365248 \\
\hline $\mathrm{s}$ & 11 & 15 & 15 & 161.505 & 0.169428 \\
\hline $\mathrm{s}$ & 11 & 15 & 16 & 104.108 & 0.144347 \\
\hline $\mathrm{s}$ & 11 & 15 & 17 & 107.016 & 0.104565 \\
\hline s & 11 & 15 & 18 & 409.557 & 0.316392 \\
\hline s & 11 & 15 & 19 & 95.7084 & 0.274343 \\
\hline $\mathrm{s}$ & 11 & 15 & 20 & 177.777 & 0.22989 \\
\hline
\end{tabular}

Sample 11 Scan 16:

\begin{tabular}{l}
\multicolumn{1}{c}{$S$} \\
238.92 \\
552.367 \\
84.4609 \\
192.064 \\
47.3386 \\
194.842 \\
813.984 \\
543.072 \\
541.632 \\
-67.1298 \\
119.728 \\
270.95 \\
131.606 \\
3846.2 \\
137.36 \\
236.882 \\
202.497 \\
97.9309 \\
281.63 \\
182.365
\end{tabular}

\begin{tabular}{ll}
\multicolumn{1}{c}{$\mathrm{t}$} & \multicolumn{1}{c}{$\mathrm{a}$} \\
1064.02 & 0.0000629101 \\
1595.52 & $-2.67937^{\prime} 10^{-6}$ \\
854.313 & 0.000125876 \\
590.018 & $-4.50452^{\prime} 10^{-6}$ \\
900.108 & $3.1364156295713237^{\prime} 10^{-6}$ \\
1000. & -0.0000134225 \\
1287.34 & $-7.12284^{\prime} 10^{-7}$ \\
904.576 & $8.05046^{\prime} 10^{-6}$ \\
1154.39 & -0.000193384 \\
1200. & $-7.40338^{\prime} 10^{-7}$ \\
1000. & $3.13609^{\prime} 10^{-6}$ \\
1529.96 & 0.0000151571 \\
1481.28 & 0.0000131952 \\
8100.08 & $5.44093^{\prime} 10^{-7}$ \\
1100. & $9.16247^{\prime} 10^{-7}$ \\
1000.04 & $-1.3351105146595675^{\prime} 10^{-7}$ \\
1588.31 & 0.0000104034 \\
1000. & $8.20315^{\prime} 10^{-6}$ \\
988.942 & $2.3110946355088825^{\prime} 10^{-6}$ \\
1000. & $-4.46311^{\prime} 10^{-6}$
\end{tabular}

$\%$ erg $f$ $\begin{array}{ll}0.706621 & -0.576577\end{array}$ $0.0908473-0.384939$ $0.0588075 \quad 1.9734$ $0.0150379 \quad 1.10098$ $0.0394072 \quad 1.26448$ $0.010458 \quad-1.4876$ $0.00586234 \quad 2.59785$ $0.00878436-1.26599$ $0.0079616-0.644591$ $0.00710454 \quad 2.02843$ $0.00460005-0.408328$ $0.00517657 \quad 0.0492046$ $0.00498366 \quad 2.52154$ $0.00150447-0.354842$ $0.00301491 \quad 0.591062$ $0.00251719-1.19392$ $0.00267723-2.98545$ $0.00427285-0.0961969$ $0.00177241 \quad 1.2388$ $0.00169709 \quad 0.0169607$

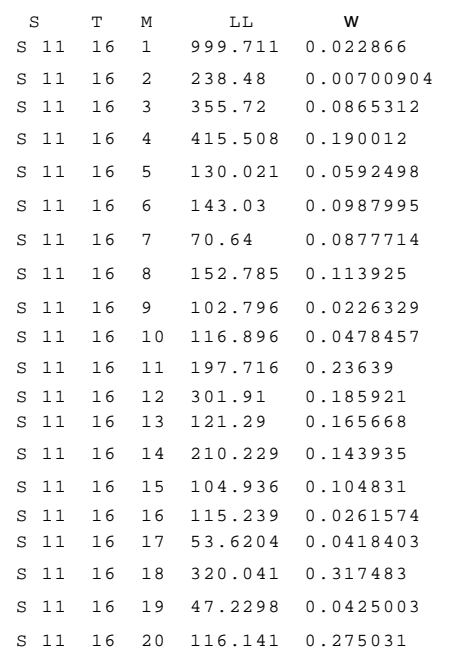
$\mathrm{s}$ 237.633 547.181 78.2303 $-48.734$ 192.22 209.807 888.335 199.48 578.628 525.138 $-56.1966$ 137.543 223.941 177.077 192.681 289.594 3440.43 165.076 1125.98 258.89

\begin{tabular}{|c|c|c|c|}
\hline $\mathrm{t}$ & a & $\frac{\circ}{\partial}$ erg & $f$ \\
\hline 1053.04 & 0.0000638671 & 0.632014 & -0.448616 \\
\hline 1593.96 & $-2.59442^{\prime} 10^{-6}$ & 0.0779409 & -0.411945 \\
\hline 852.953 & 0.000126595 & 0.0865874 & 1.85199 \\
\hline 900. & $-2.82906^{\prime} 10^{-7}$ & 0.0642272 & 1.29733 \\
\hline 588.744 & $-1.23849^{\prime} 10^{-6}$ & 0.0168808 & 0.938962 \\
\hline 1000 . & $-1.9676726385292436^{\prime} 10^{-7}$ & 0.0155133 & -1.79627 \\
\hline 1371.2 & $-6.43145^{\prime} 10^{-7}$ & 0.00723364 & 1.3342 \\
\hline 1000 & $1.65543^{\prime} 10^{-6}$ & 0.0106148 & -0.465797 \\
\hline 921.947 & $7.3880484804703234^{\prime} 10^{-6}$ & 0.0086174 & -1.72012 \\
\hline 1165.47 & -0.0000192706 & 0.00878108 & -1.38831 \\
\hline 1200 & $1.1978812205938682^{\prime} 10^{-6}$ & 0.0108423 & 2.00792 \\
\hline 1464.1 & -0.0000466571 & 0.00819237 & -0.242756 \\
\hline 1100.34 & 0 . & 0.0036404 & 0.445057 \\
\hline 900 & $-2.0662236647838533^{\prime} 10^{-6}$ & 0.00631654 & 0.558443 \\
\hline 1596.56 & $9.80051^{\prime} 10^{-6}$ & 0.00416622 & 2.28297 \\
\hline 1515.39 & 0.0000144814 & 0.00409872 & 0.321813 \\
\hline 6979.23 & -0.0000102693 & 0.00175194 & 0.57919 \\
\hline 1100. & $4.3344^{\prime} 10^{-6}$ & 0.00478604 & -0.271608 \\
\hline 1717.83 & $1.5223704550477675^{\prime} 10^{-6}$ & 0.00124639 & -2.79825 \\
\hline 10.56 & $-7.83243^{\prime} 10^{-7}$ & 0.0025767 & 1.64661 \\
\hline
\end{tabular}

\section{Sample 11 Scan 17:}

\begin{tabular}{llllll}
$S$ & $T$ & $M$ & LL & \multicolumn{1}{c}{ W } \\
$S$ & 11 & 17 & 1 & 835.034 & 0.0264598 \\
$S$ & 11 & 17 & 2 & 179.364 & 0.00699797 \\
$S$ & 11 & 17 & 3 & 440.664 & 0.0849899 \\
$S$ & 11 & 17 & 4 & 442.041 & 0.190395 \\
$S$ & 11 & 17 & 5 & 185.853 & 0.108135 \\
$S$ & 11 & 17 & 6 & 189.037 & 0.0757281 \\
$S$ & 11 & 17 & 7 & 58.1355 & 0.0481081 \\
$S$ & 11 & 17 & 8 & 120.186 & 0.197521 \\
$S$ & 11 & 17 & 9 & 105.644 & 0.0288129 \\
$S$ & 11 & 17 & 10 & 176.883 & 0.170163 \\
$S$ & 11 & 17 & 11 & 66.7957 & 0.0887352 \\
$S$ & 11 & 17 & 12 & 196.514 & 0.23632 \\
$S$ & 11 & 17 & 13 & 99.3976 & 0.0280274 \\
$S$ & 11 & 17 & 14 & 271.251 & 0.141928 \\
$S$ & 11 & 17 & 15 & 35.3678 & 0.175555 \\
$S$ & 11 & 17 & 16 & 266.782 & 0.317002 \\
$S$ & 11 & 17 & 17 & 94.2727 & 0.272692 \\
$S$ & 11 & 17 & 18 & 123.12 & 0.0610061 \\
$S$ & 11 & 17 & 19 & 60.414 & 0.301768 \\
$S$ & 11 & 17 & 20 & 64.6194 & 0.0175989
\end{tabular}

\begin{tabular}{|c|c|c|c|c|}
\hline s & $\mathrm{t}$ & a & $\div$ erg & I \\
\hline 4.132 & 1026.98 & 0.0000630025 & 0.56614 & 0.115192 \\
\hline 6.551 & 1589.51 & $-2.0868^{\prime} 10^{-6}$ & 0.0710959 & -0.447477 \\
\hline .1411 & 800. & $-2.0990844057361525^{\prime} 10^{-6}$ & 0.0667945 & 0.188013 \\
\hline .1339 & 899.975 & $3.1710065437412177^{\prime} 10^{-6}$ & 0.0924839 & 1.18776 \\
\hline 5.673 & 983.833 & $-3.74958^{\prime} 10^{-6}$ & 0.0343189 & 0.554806 \\
\hline 3.406 & 876.381 & -0.0000233979 & 0.0289418 & -0.249363 \\
\hline 1.088 & 1070.65 & $1.1124927992124435^{\prime} 10^{-6}$ & 0.00785526 & 2.16856 \\
\hline 5.863 & 1366.39 & 0.00002784 & 0.0148611 & -0.644162 \\
\hline 3.892 & 1193.33 & -0.000234775 & .0116666 & -1.01179 \\
\hline 2.462 & 1099.2 & -0.0000323995 & 0.010149 & 0.610756 \\
\hline 8.797 & 1613.79 & -0.0000136023 & 0.0061028 & -0.710263 \\
\hline 6.009 & 1200. & $3.13646^{\prime} 10^{-6}$ & 0.0123927 & 2.1194 \\
\hline 5.387 & 691.88 & -0.0000148431 & 0.00718667 & -2.00167 \\
\hline 5.358 & 899.933 & $-5.22834^{\prime} 10^{-7}$ & 0.0119234 & 0.430536 \\
\hline 16.509 & 800 . & -0.0000277703 & 0.00196867 & -1.06433 \\
\hline 8.61 & 1100 & $-7.38418^{\prime} 10^{-7}$ & 0.00707682 & -0.180733 \\
\hline 7.788 & 1000.51 & $3.5211235251907676^{\prime} 10^{-6}$ & 0.00422955 & -1.70051 \\
\hline 32.31 & 1226.41 & $7.5986230459395756^{\prime} 10^{-6}$ & 0.00504724 & 1.97858 \\
\hline 0.437 & 982.983 & $-2.92979^{\prime} 10^{-6}$ & 0.00225841 & -1.08699 \\
\hline 6.034 & 1561.8 & -0.0000178598 & 0.00226927 & -0.823936 \\
\hline
\end{tabular}


Sample 11 Scan 18:

\begin{tabular}{|c|c|c|c|c|c|c|c|c|c|c|}
\hline se & $\mathrm{s}$ & $\mathrm{T}$ & M & LL & w & $\mathrm{s}$ & $t$ & a & $\div$ erg & $f$ \\
\hline 5 & 11 & 18 & 1 & 737.05 & 0.0234598 & 236.115 & 1043.99 & 0.0000648521 & 0.521477 & -0.376162 \\
\hline 5 & 11 & 18 & 2 & 139.085 & 0.00695802 & 551.468 & 1588.17 & $-2.3041984415333526^{\prime} 10^{-6}$ & 0.0620711 & -0.432746 \\
\hline 5 & 11 & 18 & 3 & 514.404 & 0.190451 & 50.3432 & 900. & $-1.5758474662590137^{\prime} 10^{-8}$ & 0.122732 & 0.903983 \\
\hline 5 & 11 & 18 & 4 & 382.673 & 0.0868742 & 75.7408 & 848.136 & 0.000100518 & 0.0932104 & 1.95316 \\
\hline S & 11 & 18 & 5 & 80.8743 & 0.0593859 & 195.719 & 586.987 & $7.29878^{\prime} 10^{-6}$ & 0.0155332 & 0.751266 \\
\hline 5 & 11 & 18 & 6 & 133.894 & 0.099814 & 215.373 & 1000 . & $-1.94053^{\prime} 10^{-6}$ & 0.0190699 & -2.10245 \\
\hline 5 & 11 & 18 & 7 & 51.3629 & 0.0879505 & 851.494 & 1368.14 & $-6.04629^{\prime} 10^{-7}$ & 0.00827764 & 1.34468 \\
\hline D & 11 & 18 & 8 & 138.105 & 0.236395 & 234.044 & 1100 . & $5.41314^{\prime} 10^{-7}$ & 0.0146342 & 0.484025 \\
\hline $\mathrm{s}$ & 11 & 18 & 9 & 151.005 & 0.198885 & 321.09 & 1344.11 & 0.0000331802 & 0.0199614 & -2.84267 \\
\hline 5 & 11 & 18 & 10 & 102.099 & 0.111659 & 587.043 & 1235.9 & $6.36385^{\prime} 10^{-6}$ & 0.0118861 & -2.55586 \\
\hline 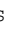 & 11 & 18 & 11 & 174.533 & 0.144795 & 145.48 & 900.003 & $-7.59393^{\prime} 10^{-6}$ & 0.0142793 & 0.0799888 \\
\hline S & 11 & 18 & 12 & 82.933 & 0.022703 & 557.851 & 920.152 & $7.70848^{\prime} 10^{-6}$ & 0.00766387 & -1.84277 \\
\hline $\mathrm{s}$ & 11 & 18 & 13 & 100.978 & 0.0478289 & 517.376 & 1161.11 & -0.0000190682 & 0.00851276 & -1.41318 \\
\hline $\mathrm{s}$ & 11 & 18 & 14 & 108.254 & 0.305129 & 265.418 & 938.94 & -0.0000186935 & 0.00821664 & -0.643956 \\
\hline $\mathrm{s}$ & 11 & 18 & 15 & 40.8446 & 0.209164 & 397.24 & 846.073 & -0.0000189975 & 0.00287764 & 2.38674 \\
\hline s & 11 & 18 & 16 & 118.226 & 0.170736 & 379.315 & 1097.39 & -0.000014565 & 0.00769655 & 0.710908 \\
\hline $\mathrm{s}$ & 11 & 18 & 17 & 110.459 & 0.272247 & 240.07 & 1000 . & $3.13234^{\prime} 10^{-6}$ & 0.00583684 & -1.89068 \\
\hline S & 11 & 18 & 18 & 75.3242 & 0.327938 & 213.429 & 900. & $3.13609^{\prime} 10^{-6}$ & 0.00390519 & 1.39373 \\
\hline$S$ & 11 & 18 & 19 & 47.3818 & 0.103574 & 417.416 & 742.838 & $-3.3250444852678647^{\prime} 10^{-6}$ & 0.00238722 & 1.62421 \\
\hline $\mathrm{s}$ & 11 & 18 & 20 & 78.2589 & 0.171041 & 923.763 & 1890. & 0.0000458124 & 0.00370342 & 0.486603 \\
\hline
\end{tabular}

\section{Sample 11 Scan 19:}

\begin{tabular}{llllll}
$S$ & $T$ & $M$ & LL & \multicolumn{1}{c}{$W$} \\
$S$ & 11 & 19 & 1 & 727.311 & 0.0264698 \\
$S$ & 11 & 19 & 2 & 141.685 & 0.00697084 \\
$S$ & 11 & 19 & 3 & 453.871 & 0.190752 \\
$S$ & 11 & 19 & 4 & 343.527 & 0.084197 \\
$S$ & 11 & 19 & 5 & 287.964 & 0.107039 \\
$S$ & 11 & 19 & 6 & 84.1834 & 0.0607951 \\
$S$ & 11 & 19 & 7 & 110.044 & 0.193823 \\
$S$ & 11 & 19 & 8 & 139.809 & 0.236574 \\
$S$ & 11 & 19 & 9 & 56.9555 & 0.0254959 \\
$S$ & 11 & 19 & 10 & 76.9911 & 0.100126 \\
$S$ & 11 & 19 & 11 & 221.713 & 0.14357 \\
$S$ & 11 & 19 & 12 & 128.934 & 0.170187 \\
$S$ & 11 & 19 & 13 & 47.7806 & 0.0164198 \\
$S$ & 11 & 19 & 14 & 129.408 & 0.0464999 \\
$S$ & 11 & 19 & 15 & 161.949 & 0.109271 \\
$S$ & 11 & 19 & 16 & 110.49 & 0.30507 \\
$S$ & 11 & 19 & 17 & 121.055 & 0.0828033 \\
$S$ & 11 & 19 & 18 & 90.4192 & 0.272988 \\
$S$ & 11 & 19 & 19 & 131.744 & 0.228429 \\
$S$ & 11 & 19 & 20 & 84.2759 & 0.327489
\end{tabular}

\begin{tabular}{|c|c|c|c|c|}
\hline s & $\mathrm{t}$ & a & $\therefore$ erg & $f$ \\
\hline 4.572 & 24.19 & 0.0000635056 & 0.516794 & 0.10813 \\
\hline 522.48 & 1583.84 & $-2.14937^{\prime} 10^{-6}$ & 0.0637342 & -0.423824 \\
\hline 57.1753 & 900. & $2.9293546022344665^{\prime} 10^{-6}$ & 0.12084 & 0.876936 \\
\hline 114.707 & 865.479 & 0.0000102814 & 0.0725138 & 0.662676 \\
\hline 74.798 & 1100 . & $3.5448249234734407^{\prime} 10^{-8}$ & 0.0355775 & 0.212254 \\
\hline 54.986 & 568.001 & $-6.0229613858947164^{\prime} 10^{-6}$ & 0.0152796 & 1.91846 \\
\hline 41.691 & 1457.26 & 0.0000112098 & 0.018163 & 0.485845 \\
\hline 83.1887 & 1200. & $3.9912^{\prime} 10^{-8}$ & 0.0202698 & 1.64244 \\
\hline 621.775 & 916.595 & $-2.11903^{\prime} 10^{-6}$ & 0.00752217 & -1.51761 \\
\hline 433.285 & 1363.81 & -0.0000156976 & 0.00951063 & 1.15419 \\
\hline 133.515 & 900.001 & $-8.21821 \cdot 10^{-6}$ & 0.0204986 & 0.16677 \\
\hline 166.546 & 1099.92 & $-4.2499733637956934^{\prime} 10^{-6}$ & 0.00805318 & 0.116903 \\
\hline 728.456 & 1281.74 & $-2.95603^{\prime} 10^{-6}$ & 0.00421234 & -2.67406 \\
\hline 445.575 & 1134.62 & -0.0000179543 & 0.0104477 & -0.291479 \\
\hline 201.01 & 799.831 & $-1.1918589695221292^{\prime} 10^{-6}$ & 0.00639059 & 0.486753 \\
\hline 46.964 & 900 & -0.0000209229 & 0.0072074 & -1.22944 \\
\hline 3.049 & 1521.61 & -0.0000288835 & 0.00707173 & 0.946793 \\
\hline 33.074 & 989.782 & $3.2952071384520146^{\prime} 10^{-6}$ & 0.0047508 & 0.791169 \\
\hline 28 & .926 & $-4.97388^{\prime} 10^{-6}$ & 0.003226 & -0 \\
\hline .184 & 900.097 & $1.3013716645763958^{\prime} 10^{-6}$ & 0.00365806 & 1.37474 \\
\hline
\end{tabular}

Sample 11 Scan 20:

\begin{tabular}{llllll}
\multicolumn{1}{c}{$S$} & T & $M$ & \multicolumn{1}{c}{ LL } & \multicolumn{1}{c}{$W$} \\
$S$ & 11 & 20 & 1 & 714.89 & 0.0264872 \\
$S$ & 11 & 20 & 2 & 139.617 & 0.00697183 \\
$S$ & 11 & 20 & 3 & 424.574 & 0.190055 \\
$S$ & 11 & 20 & 4 & 363.669 & 0.0911036 \\
$S$ & 11 & 20 & 5 & 93.5614 & 0.0980877 \\
$S$ & 11 & 20 & 6 & 83.8694 & 0.059838 \\
$S$ & 11 & 20 & 7 & 41.1559 & 0.0876661 \\
$S$ & 11 & 20 & 8 & 121.945 & 0.194279 \\
$S$ & 11 & 20 & 9 & 104.129 & 0.112233 \\
$S$ & 11 & 20 & 10 & 185.671 & 0.236141 \\
$S$ & 11 & 20 & 11 & 252.181 & 0.145529 \\
$S$ & 11 & 20 & 12 & 86.3551 & 0.0290102 \\
$S$ & 11 & 20 & 13 & 156.774 & 0.178014 \\
$S$ & 11 & 20 & 14 & 110.038 & 0.0304948 \\
$S$ & 11 & 20 & 15 & 95.1409 & 0.304922 \\
$S$ & 11 & 20 & 16 & 72.1891 & 0.273148 \\
$S$ & 11 & 20 & 17 & 136.428 & 0.0584097 \\
$S$ & 11 & 20 & 18 & 56.0201 & 0.207069 \\
$S$ & 11 & 20 & 19 & 62.8664 & 0.229362 \\
$S$ & 11 & 20 & 20 & 92.1168 & 0.327557
\end{tabular}

\begin{tabular}{|c|c|c|c|c|}
\hline s & $\mathrm{t}$ & a & $\frac{\circ}{\partial}$ erg & $f$ \\
\hline 224.26 & 1024.28 & 0.000063392 & 0.510754 & 0.112911 \\
\hline 521.57 & 1584.05 & $-2.1087769073912414^{\prime} 10^{-6}$ & 0.0636547 & -0.426592 \\
\hline 59.0421 & 899.998 & $-1.05746^{\prime} 10^{-9}$ & 0.11944 & 0.927283 \\
\hline 72.917 & 841.81 & 0.0000977525 & 0.093046 & 2.51003 \\
\hline 221.686 & 1000 & $3.17664^{\prime} 10^{-6}$ & 0.0156737 & -2.37921 \\
\hline 186.466 & 582.982 & $3.6633550656149212^{\prime} 10^{-6}$ & 0.0158058 & 0.993764 \\
\hline 964.945 & 1397.07 & $-6.6818^{\prime} 10^{-7}$ & 0.0072845 & -1.28231 \\
\hline 272.931 & 1442.56 & 0.0000112827 & 0.0199046 & -2.80931 \\
\hline 557.44 & 1194.49 & $6.27368^{\prime} 10^{-6}$ & 0.0151773 & 2.14852 \\
\hline 75.0746 & 1199.69 & $5.26571^{\prime} 10^{-7}$ & 0.0226175 & 1.76212 \\
\hline 151.191 & 900. & $2.0719540810980123^{\prime} 10^{-8}$ & 0.0186814 & 0.255244 \\
\hline 99.7892 & 1136.55 & 0.0000451008 & 0.00802491 & -0.604703 \\
\hline 136.794 & 1100 & $1.5304281985428763^{\prime} 10^{-7}$ & 0.00886159 & 0.400534 \\
\hline 244.503 & 724.716 & -0.000027887 & 0.00834691 & -2.83494 \\
\hline 250.079 & 900.107 & -0.0000169639 & 0.00649472 & -1.31997 \\
\hline 320.028 & 998.815 & $1.8269148263258746^{\prime} 10^{-6}$ & 0.00454506 & -1.56501 \\
\hline 126.082 & 1391.4 & 0.000130526 & 0.00774319 & -1.56058 \\
\hline 255.528 & 765.837 & $-1.34087^{\prime} 10^{-6}$ & 0.00288562 & -0.249399 \\
\hline 363.632 & 860.542 & $-4.9686^{\prime} 10^{-6}$ & 0.0030515 & 0.18154 \\
\hline 215.261 & 900.615 & $6.56472^{\prime} 10^{-6}$ & 0.0037659 & 1.24966 \\
\hline
\end{tabular}


Sample 11 Scan 21:

\begin{tabular}{|c|c|c|c|c|c|c|c|c|c|c|}
\hline $\mathrm{s}$ & & $\mathrm{T}$ & M & LL & w & $\mathrm{s}$ & $t$ & a & $\div$ erg & $f$ \\
\hline $\mathrm{s}$ & 11 & 21 & 1 & 723.156 & 0.0235331 & 235.576 & 1043.6 & 0.0000645272 & 0.514781 & -0.356733 \\
\hline s & 11 & 21 & 2 & 136.649 & 0.00695427 & 549.354 & 1582.98 & $-2.46538^{\prime} 10^{-6}$ & 0.0619123 & -0.378041 \\
\hline $\mathrm{s}$ & 11 & 21 & 3 & 148.803 & 0.189421 & 235.545 & 900. & $-1.0803899646330207^{\prime} 10^{-7}$ & 0.0558146 & 0.965684 \\
\hline $\mathrm{s}$ & 11 & 21 & 4 & 309.223 & 0.0904401 & 70.8221 & 845.022 & 0.000130049 & 0.0975956 & 2.18054 \\
\hline $\mathrm{s}$ & 11 & 21 & 5 & 248.968 & 0.166688 & 57.2468 & 900. & $-1.9087^{\prime} 10^{-6}$ & 0.0508732 & 0.821159 \\
\hline s & 11 & 21 & 6 & 74.5235 & 0.193185 & 503.186 & 989.006 & -0.000012368 & 0.0156883 & -0.420209 \\
\hline $\mathrm{s}$ & 11 & 21 & 7 & 80.978 & 0.0596829 & 186.914 & 580.743 & $2.1998^{\prime} 10^{-6}$ & 0.0157725 & 1.14727 \\
\hline $\mathrm{s}$ & 11 & 21 & 8 & 124.678 & 0.0987433 & 201.009 & 1000 . & 0 . & 0.0159854 & -2.23565 \\
\hline $\mathrm{S}$ & 11 & 21 & 9 & 52.3192 & 0.0875308 & 883.143 & 1310.87 & $-3.8706518495779906^{\prime} 10^{-7}$ & 0.00871706 & -0.0463887 \\
\hline $\mathrm{s}$ & 11 & 21 & 10 & 94.0778 & 0.189064 & 185.19 & 1508.4 & -0.0000221516 & 0.0145719 & -2.79106 \\
\hline s & 11 & 21 & 11 & 152.905 & 0.211582 & 203.613 & 800.096 & $4.00776^{\prime} 10^{-6}$ & 0.0114632 & -2.71519 \\
\hline s & 11 & 21 & 12 & 154.623 & 0.114483 & 220.653 & 1000.12 & $-7.50082^{\prime} 10^{-10}$ & 0.013699 & -0.830136 \\
\hline S & 11 & 21 & 13 & 233.11 & 0.233393 & 74.875 & 1199.67 & $1.1744222105791696^{\prime} 10^{-6}$ & 0.0241513 & 1.80901 \\
\hline s & 11 & 21 & 14 & 63.9702 & 0.167006 & 351.239 & 923.235 & $-4.06896^{\prime} 10^{-6}$ & 0.00609799 & -1.83362 \\
\hline s & 11 & 21 & 15 & 84.9819 & 0.0226641 & 559.367 & 921.573 & $7.72452^{\prime} 10^{-6}$ & 0.00752051 & -1.88211 \\
\hline s & 11 & 21 & 16 & 111.611 & 0.0476513 & 508.75 & 1158.39 & -0.0000190032 & 0.00895432 & -1.27913 \\
\hline S & 11 & 21 & 17 & 211.986 & 0.305251 & 98.2398 & 1000 . & $-3.26704^{\prime} 10^{-7}$ & 0.00821781 & -0.481859 \\
\hline $\mathrm{s}$ & 11 & 21 & 18 & 95.5517 & 0.105243 & 201.408 & 1598.96 & 0.0000124409 & 0.00616178 & 1.66397 \\
\hline S & 11 & 21 & 19 & 137.517 & 0.145785 & 166.733 & 900. & $5.07462^{\prime} 10^{-6}$ & 0.00526804 & -0.221498 \\
\hline & 11 & 21 & 20 & 72.0064 & 0.272542 & 334.724 & 948.247 & $7.73174^{\prime} 10^{-6}$ & 0.00390356 & -0.471381 \\
\hline
\end{tabular}

\section{Sample 11 Scan 22:}

\begin{tabular}{|c|c|c|c|c|c|c|c|c|c|c|}
\hline $\mathrm{s}$ & & $\mathrm{T}$ & M & LL & w & $\mathrm{s}$ & $t$ & a & $\div$ erg & $f$ \\
\hline & 11 & 22 & 1 & 705.752 & 0.0265198 & 223.554 & 1024.3 & 0.0000630681 & 0.506263 & 0.12874 \\
\hline S & 11 & 22 & 2 & 138.22 & 0.0069746 & 521.974 & 1581.4 & $-2.09736^{\prime} 10^{-6}$ & 0.0636436 & -0.39712 \\
\hline $\mathrm{s}$ & 11 & 22 & 3 & 464.569 & 0.189217 & 48.606 & 900. & $-4.44794^{\prime} 10^{-7}$ & 0.12778 & 0.998207 \\
\hline & 11 & 22 & 4 & 380.894 & 0.0905089 & 75.8541 & 841.616 & 0.0000870454 & 0.0954655 & 2.56443 \\
\hline S & 11 & 22 & 5 & 120.043 & 0.0981628 & 208.188 & 1000.02 & $-1.9382165747768534^{\prime} 10^{-6}$ & 0.0165601 & -2.26665 \\
\hline $\mathrm{s}$ & 11 & 22 & 6 & 88.2245 & 0.0600217 & 177.348 & 576.398 & $1.6182115434345117^{\prime} 10^{-6}$ & 0.0159908 & 1.39354 \\
\hline s & 11 & 22 & 7 & 49.2336 & 0.0875363 & 906.473 & 1339.29 & $-4.7414^{\prime} 10^{-7}$ & 0.00832912 & -2.53128 \\
\hline s & 11 & 22 & 8 & 201.915 & 0.236527 & 45.0344 & 1199.77 & $2.7054129833592705^{\prime} 10^{-6}$ & 0.0295089 & 1.72178 \\
\hline $\mathrm{s}$ & 11 & 22 & 9 & 115.918 & 0.112295 & 597.497 & 1182.47 & $6.4259^{\prime} 10^{-6}$ & 0.0148346 & -2.82091 \\
\hline S & 11 & 22 & 10 & 128.807 & 0.191905 & 161.872 & 1504.89 & $6.3227^{\prime} 10^{-6}$ & 0.0145875 & -2.2584 \\
\hline S & 11 & 22 & 11 & 71.6243 & 0.0255193 & 646.744 & 929.564 & $-2.11825^{\prime} 10^{-6}$ & 0.00733454 & -1.83006 \\
\hline $\mathrm{s}$ & 11 & 22 & 12 & 183.141 & 0.145305 & 159.174 & 900. & $8.20327^{\prime} 10^{-6}$ & 0.0118516 & -0.0286868 \\
\hline s & 11 & 22 & 13 & 49.996 & 0.0163366 & 707.745 & 1267 . & $-3.09755^{\prime} 10^{-6}$ & 0.00423918 & -2.41808 \\
\hline s & 11 & 22 & 14 & 98.2631 & 0.0394824 & 250.666 & 947.735 & -0.0000119088 & 0.00768293 & 1.20153 \\
\hline s & 11 & 22 & 15 & 133.671 & 0.23144 & 251.521 & 1000 . & $-1.8693634433225678^{\prime} 10^{-6}$ & 0.00544073 & -0.548325 \\
\hline s & 11 & 22 & 16 & 75.2733 & 0.167773 & 387.473 & 999.588 & -0.000013653 & 0.00504414 & -1.62984 \\
\hline s & 11 & 22 & 17 & 73.0515 & 0.275475 & 128.73 & 1100. & $4.38285^{\prime} 10^{-6}$ & 0.00434242 & 1.78987 \\
\hline s & 11 & 22 & 18 & 97.2654 & 0.304585 & 230.043 & 899.93 & -0.0000104401 & 0.00522427 & -1.3077 \\
\hline $\mathrm{s}$ & 11 & 22 & 19 & 120.275 & 0.0608668 & 114.039 & 1377.39 & 0.0000743783 & 0.00622758 & -0.532229 \\
\hline$S$ & 11 & 22 & 20 & 67.3545 & 0.10201 & 970.75 & 1205.28 & $3.9315^{\prime} 10^{-6}$ & 0.00317385 & -3.08012 \\
\hline
\end{tabular}

Sample 11 Scan 23:

\begin{tabular}{|c|c|c|c|c|c|c|c|c|c|c|}
\hline$s$ & & $\mathrm{~T}$ & M & LL & w & $\mathrm{s}$ & $\mathrm{t}$ & a & $\frac{\circ}{6}$ erg & $f$ \\
\hline 5 & 11 & 23 & 1 & 708.55 & 0.0265481 & 223.057 & 1023.83 & 0.0000631046 & 0.507642 & 0.132202 \\
\hline & 11 & 23 & 2 & 139.256 & 0.00695688 & 522.986 & 1580.33 & $-2.0109921389523017^{\prime} 10^{-6}$ & 0.0639103 & -0.401891 \\
\hline & 11 & 23 & 3 & 283.266 & 0.18772 & 126.207 & 870.854 & $-3.58034^{\prime} 10^{-6}$ & 0.0753409 & 0.161033 \\
\hline & 11 & 23 & 4 & 332.606 & 0.0922846 & 69.2238 & 839.323 & 0.0000652831 & 0.0996514 & 2.78426 \\
\hline & 11 & 23 & 5 & 75.8413 & 0.0975708 & 206.03 & 999.926 & $-8.78212^{\prime} 10^{-10}$ & 0.0153387 & -2.42595 \\
\hline & 11 & 23 & 6 & 169.892 & 0.164737 & 267.877 & 1000.05 & $-9.50752^{\prime} 10^{-6}$ & 0.0252992 & -2.67209 \\
\hline & 11 & 23 & 7 & 78.4387 & 0.0597781 & 160.268 & 564.748 & $-3.04693^{\prime} 10^{-6}$ & 0.0159852 & 2.12262 \\
\hline & 11 & 23 & 8 & 100.736 & 0.211837 & 227.003 & 866.274 & $4.11073^{\prime} 10^{-6}$ & 0.018246 & 2.31259 \\
\hline & 11 & 23 & 9 & 110.924 & 0.193758 & 292.43 & 1443.35 & 0.0000122675 & 0.0186541 & -2.93197 \\
\hline & 11 & 23 & 10 & 52.7162 & 0.0874686 & 909.656 & 1337.73 & $-4.2199389533461346^{\prime} 10^{-7}$ & 0.00816561 & -2.41903 \\
\hline & 11 & 23 & 11 & 106.773 & 0.111745 & 588.841 & 1232.08 & $6.52769^{\prime} 10^{-6}$ & 0.0152767 & -2.04583 \\
\hline$S$ & 11 & 23 & 12 & 215.992 & 0.233144 & 78.6675 & 1200. & $1.4347319027257846^{\prime} 10^{-7}$ & 0.0242121 & 1.87512 \\
\hline S & 11 & 23 & 13 & 256.545 & 0.145174 & 168.126 & 900. & $-1.9382118553057415^{\prime} 10^{-6}$ & 0.0192284 & 0.389066 \\
\hline & 11 & 23 & 14 & 84.542 & 0.0255113 & 637.935 & 920.997 & $-1.98182^{\prime} 10^{-6}$ & 0.00746898 & -1.64639 \\
\hline & 11 & 23 & 15 & 202.844 & 0.176835 & 189.898 & 737.049 & $-7.76377^{\prime} 10^{-7}$ & 0.00797199 & -3.00851 \\
\hline 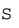 & 11 & 23 & 16 & 154.383 & 0.0455432 & 457.8 & 1110. & -0.0000174396 & 0.0109713 & 0.778862 \\
\hline & 11 & 23 & 17 & 67.2706 & 0.0163369 & 718.902 & 1268.89 & $-3.1312022205436967^{\prime} 10^{-6}$ & 0.00427566 & -2.45336 \\
\hline & 11 & 23 & 18 & 175.916 & 0.304768 & 101.6 & 1000 & $2.83627^{\prime} 10^{-6}$ & 0.00598929 & -0.403741 \\
\hline & 11 & 23 & 19 & 68.6612 & 0.101825 & 883.075 & 1214.15 & $4.4486328325134135^{\prime} 10^{-6}$ & 0.00367989 & 2.09735 \\
\hline & 11 & 23 & 20 & 44.3783 & 0.128195 & 281.45 & 941.597 & $7.64099^{\prime} 10^{-6}$ & 0.00224742 & 0.219774 \\
\hline
\end{tabular}


Sample 11 Scan 24:

\begin{tabular}{|c|c|c|c|c|c|c|c|c|c|c|}
\hline $\mathrm{s}$ & & $\mathrm{T}$ & M & LL & w & $\mathrm{s}$ & $t$ & a & $\div$ erg & $f$ \\
\hline & 11 & 24 & 1 & 698.076 & 0.0265475 & 223.495 & 1023.45 & 0.0000631244 & 0.502458 & 0.131445 \\
\hline s & 11 & 24 & 2 & 136.299 & 0.00695015 & 523.84 & 1578.84 & $-2.0381542763241603^{\prime} 10^{-6}$ & 0.0633034 & -0.39114 \\
\hline $\mathrm{s}$ & 11 & 24 & 3 & 434.141 & 0.190007 & 63.3593 & 900. & $3.13512^{\prime} 10^{-6}$ & 0.119358 & 0.977703 \\
\hline $\mathrm{s}$ & 11 & 24 & 4 & 386.892 & 0.0920834 & 71.0234 & 839.973 & 0.0000868718 & 0.100732 & 2.67203 \\
\hline $\mathrm{s}$ & 11 & 24 & 5 & 94.9023 & 0.0979942 & 233.411 & 1000 . & $3.1353149931930074^{\prime} 10^{-6}$ & 0.0147031 & -2.45705 \\
\hline s & 11 & 24 & 6 & 86.0936 & 0.0600021 & 172.087 & 572.77 & $1.78908^{\prime} 10^{-6}$ & 0.0163771 & 1.59625 \\
\hline $\mathrm{s}$ & 11 & 24 & 7 & 91.8548 & 0.111936 & 560.819 & 1210.9 & $6.6287324327986806^{\prime} 10^{-6}$ & 0.0159863 & 0.266406 \\
\hline $\mathrm{s}$ & 11 & 24 & 8 & 129.893 & 0.194245 & 282.968 & 1438.48 & 0.0000168405 & 0.0202411 & -2.07049 \\
\hline $\mathrm{s}$ & 11 & 24 & 9 & 41.7838 & 0.0868282 & 331.422 & 1614.68 & -0.0000173676 & 0.00597177 & -0.9254 \\
\hline S & 11 & 24 & 10 & 216.187 & 0.235395 & 96.0347 & 1200 . & $1.1978812205938682^{\prime} 10^{-6}$ & 0.0252787 & 1.85082 \\
\hline $\mathrm{s}$ & 11 & 24 & 11 & 66.3931 & 0.0255355 & 643.741 & 922.967 & $-2.0497863828243496^{\prime} 10^{-6}$ & 0.00736651 & -1.69521 \\
\hline $\mathrm{s}$ & 11 & 24 & 12 & 234.747 & 0.14554 & 138.174 & 900.108 & $-1.8902188479974533^{\prime} 10^{-6}$ & 0.0191302 & 0.251345 \\
\hline $\mathrm{s}$ & 11 & 24 & 13 & 189.997 & 0.170334 & 180.753 & 1100. & -0.0000240694 & 0.00878325 & 0.236698 \\
\hline s & 11 & 24 & 14 & 54.3294 & 0.0163043 & 739.175 & 1269.71 & $-2.9337664868177027^{\prime} 10^{-6}$ & 0.00419849 & -2.48928 \\
\hline $\mathrm{s}$ & 11 & 24 & 15 & 153.8 & 0.0453161 & 463.736 & 1107.41 & -0.0000168251 & 0.01072 & 0.835429 \\
\hline s & 11 & 24 & 16 & 45.242 & 0.104285 & 664.784 & 929.736 & $1.7923015628449305^{\prime} 10^{-6}$ & 0.00285212 & 0.315022 \\
\hline S & 11 & 24 & 17 & 70.9366 & 0.0876626 & 424.628 & 922.84 & $-5.99631 \cdot 10^{-6}$ & 0.0042201 & 2.69966 \\
\hline $\mathrm{s}$ & 11 & 24 & 18 & 61.2429 & 0.206556 & 220.5 & 739.172 & 0.0000117929 & 0.0034102 & -1.15301 \\
\hline s & 11 & 24 & 19 & 164.093 & 0.303671 & 109.452 & 1000 . & $4.9663^{\prime} 10^{-6}$ & 0.00529525 & -0.51401 \\
\hline $\mathrm{s}$ & 11 & 24 & 20 & 52.7237 & 0.132413 & 405.111 & 1734.25 & -0.000127996 & 0.00250116 & -0.246163 \\
\hline
\end{tabular}

\section{Sample 11 Scan 25:}

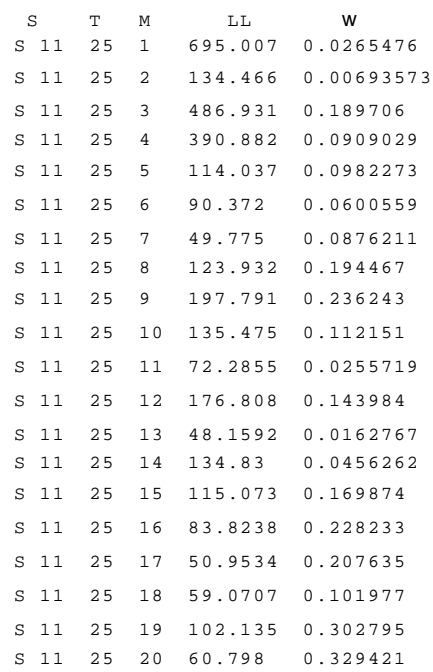

$\mathrm{s}$

223.82

524.573

46.2674

75.4475

215.093

170.8

941.071

287.77

97.3157

608.545

649.015

153.546

729.464

463.842

$-97.8564$

243.031

308.522

935.552

179.565

243.45

\section{Sample 11 Scan 26:}

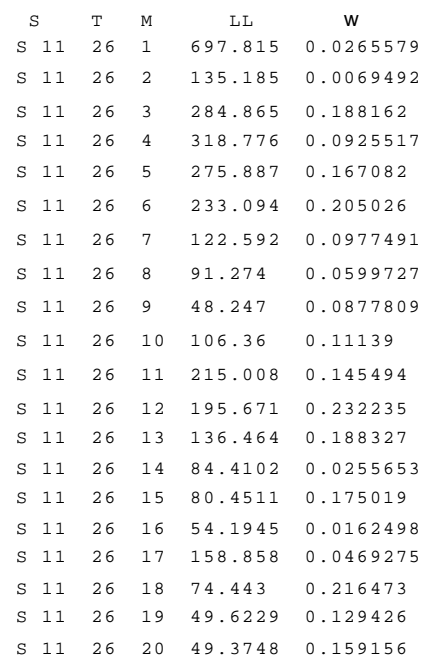

\begin{tabular}{|c|c|c|c|c|}
\hline $\mathrm{s}$ & $\mathrm{t}$ & a & $\div$ erg & f \\
\hline 935 & 1023.32 & 0.0000633817 & 0.502329 & 0.129637 \\
\hline .535 & 1577.86 & $-2.0654947151701003^{\prime} 10^{-6}$ & 0.0628389 & -0.385243 \\
\hline .939 & 1007.27 & $-9.38602^{\prime} 10^{-6}$ & 0.0561982 & -0.189666 \\
\hline .708 & 839.254 & 0.0000715555 & 0.103111 & 2.75656 \\
\hline 5.974 & 1000 . & $-1.9706084752615495^{\prime} 10^{-6}$ & 0.0411435 & -2.83636 \\
\hline .712 & 1000 & $-1.1993333311115945^{\prime} 10^{-6}$ & 0.0305224 & -0.694294 \\
\hline 905 & 1000 . & $2.17002^{\prime} 10^{-8}$ & 0.0146079 & -2.46325 \\
\hline .983 & 564.928 & $1.2131117713587844^{\prime} 10^{-6}$ & 0.0164312 & 2.07948 \\
\hline 016 & 1406.23 & $-8.69683^{\prime} 10^{-7}$ & 0.00809829 & -2.00152 \\
\hline .694 & 1251.87 & $6.79847^{\prime} 10^{-6}$ & 0.0165307 & 1.95177 \\
\hline .398 & 899.933 & $5.05039^{\prime} 10^{-9}$ & 0.0193408 & 0.394973 \\
\hline 119 & 1200 . & $5.07201^{\prime} 10^{-6}$ & 0.0199936 & 1.95152 \\
\hline .897 & 1506.46 & -0.0000139816 & 0.0137727 & -2.40713 \\
\hline .75 & 923.551 & $-2.00514^{\prime} 10^{-6}$ & 0.00762475 & -1.72997 \\
\hline .071 & 870.589 & -0.0000138021 & 0.00669191 & -0.660531 \\
\hline .258 & 1254.61 & $-3.13158^{\prime} 10^{-6}$ & 0.00421378 & -2.21307 \\
\hline .205 & 1148.19 & -0.0000180086 & 0.0111139 & -0.968204 \\
\hline .48 & 950.051 & $5.0820685508906234^{\prime} 10^{-6}$ & 0.00463087 & 2.44872 \\
\hline .32 & 926.132 & 0.000011693 & 0.00290085 & 2.21688 \\
\hline & 031.9 & $-2.4089808191081052^{\prime} 10^{-6}$ & 0.00274695 & 0.368922 \\
\hline
\end{tabular}




\section{Sample 11 Scan 27:}

\begin{tabular}{|c|c|c|c|c|c|c|c|c|c|c|}
\hline$S$ & & T & M & LL & w & $\mathrm{s}$ & 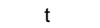 & $\mathrm{a}$ & $\therefore$ erg & $f^{\prime}$ \\
\hline $\mathrm{s}$ & 11 & 27 & 1 & 708.826 & .0265504 & 224.229 & 1023.27 & 0.0000637223 & 0.507778 & 0.118562 \\
\hline & 11 & 27 & 2 & 138.594 & 0.0069538 & 521.874 & 1577.42 & $-2.0305907309365547^{\prime} 10^{-6}$ & 0.06361 & -0.395641 \\
\hline & 11 & 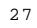 & 3 & 1.664 & .092787 & .5406 & 8.659 & 0.0000704157 & .10499 & 2.80851 \\
\hline & 11 & 27 & 4 & 679.498 & .182776 & 6.6244 & 917.225 & -0.0000309866 & 0.12645 & -2.10419 \\
\hline & 11 & 27 & 5 & 120.489 & 0.0979746 & 201.306 & 1000 . & $6.85866^{\prime} 10^{-8}$ & 0.0149318 & -2.41038 \\
\hline & 11 & 27 & 6 & 98.8354 & 0.0598325 & 162.248 & 563.959 & $2.7123542868067893^{\prime} 10^{-7}$ & 0.0170648 & 2.14392 \\
\hline & 11 & 27 & 7 & 56.782 & 0.0874985 & 935.991 & 1338.54 & $-5.16077^{\prime} 10^{-7}$ & 0.00906769 & -2.4849 \\
\hline & 11 & 27 & 8 & 113.53 & 0.11176 & 647.43 & 212.23 & $6.75349^{\prime} 10^{-6}$ & 0.0166567 & 0.0743585 \\
\hline & 11 & 27 & 9 & 153.167 & 0.194875 & 401.439 & 1411.29 & 0.0000131799 & 0.0196753 & -2.88921 \\
\hline & 11 & 27 & 10 & 67.9071 & 0.0256188 & 647.329 & 923.101 & $-1.9148097279193726^{\prime} 10^{-6}$ & 0.00780412 & -1.73663 \\
\hline & 11 & 27 & 11 & 126.952 & 0.169438 & 412.198 & 1025.5 & -0.0000126513 & 0.0132417 & 0.31891 \\
\hline & 11 & 27 & 12 & 178.607 & 0.224175 & 66.0293 & 1200. & $3.4188751470306183^{\prime} 10^{-6}$ & 0.0149634 & 1.83759 \\
\hline & 11 & 27 & 13 & 54.2528 & 0.0162871 & 737.881 & 259.84 & $-3.1293437886512886^{\prime} 10^{-6}$ & 0.00437562 & -2.30776 \\
\hline & 11 & 27 & 14 & 158.504 & 0.0468659 & 460.798 & 1147.36 & -0.0000177545 & 0.0115043 & -0.944431 \\
\hline & 11 & 27 & 15 & 57.7117 & 0.101734 & 882.048 & 1228.78 & $4.6201021383778774^{\prime} 10^{-6}$ & 0.00375612 & 0.572394 \\
\hline & 11 & 27 & 16 & .0172 & 18019 & .944 & 218.59 & $2.25915^{\prime} 10^{-6}$ & 0.00409824 & 1.12961 \\
\hline & 11 & 27 & 17 & 99.2587 & 0.302561 & 542 & 900.19 & -0.0000238779 & 0.00548766 & -1.43528 \\
\hline S & 11 & 27 & 18 & 90.5433 & 0.243698 & 209.557 & 1100 . & $1.9382125294060955^{\prime} 10^{-6}$ & 0.0041081 & 1.20155 \\
\hline & 11 & 27 & 19 & 48.4323 & 0.197881 & .765 & 5.532 & 33357 & 0.00234169 & 0.470482 \\
\hline & & & & & 329978 & 9.152 & 0.108 & ' & 0.00253025 & .4002 \\
\hline
\end{tabular}

\section{Sample 11 Scan 28:}

\begin{tabular}{|c|c|c|c|c|c|c|c|c|c|c|}
\hline s & & $\mathrm{T}$ & M & LL & w & s & $\mathrm{t}$ & a & $\frac{\circ}{\partial}$ erg & $f$ \\
\hline $\mathrm{s}$ & 11 & 28 & 1 & 693.066 & 0.026613 & 223.368 & 1023.08 & 0.0000635131 & 0.499959 & 0.131726 \\
\hline $\mathrm{s}$ & 11 & 28 & 2 & 135.002 & 0.00697147 & 519.754 & 1578.58 & $-1.99604^{\prime} 10^{-6}$ & 0.0630581 & -0.405254 \\
\hline $\mathrm{s}$ & 11 & 28 & 3 & 430.79 & 0.186643 & 55.999 & 908.631 & -0.0000239783 & 0.12731 & -0.505597 \\
\hline $\mathrm{s}$ & 11 & 28 & 4 & 411.473 & 0.091889 & 73.4724 & 840.074 & 0.0000793544 & 0.104156 & 2.6926 \\
\hline s & 11 & 28 & 5 & 126.582 & 0.0981119 & 204.065 & 999.999 & $5.94061^{\prime} 10^{-9}$ & 0.016349 & -2.35796 \\
\hline $\mathrm{s}$ & 11 & 28 & 6 & 94.4714 & 0.0600384 & 163.897 & 568.079 & $-5.5470182837932873^{\prime} 10^{-8}$ & 0.0169712 & 1.90337 \\
\hline $\mathrm{s}$ & 11 & 28 & 7 & 55.5214 & 0.0881068 & 190.37 & 1634.86 & -0.000175974 & 0.00925118 & -2.08204 \\
\hline$S$ & 11 & 28 & 8 & 125.913 & 0.194207 & 311.409 & 1435.15 & 0.0000138432 & 0.0191708 & -1.1895 \\
\hline $\mathrm{s}$ & 11 & 28 & 9 & 60.0984 & 0.0254521 & 694.194 & 976.594 & $-1.94846^{\prime} 10^{-6}$ & 0.00833333 & -2.99269 \\
\hline $\mathrm{s}$ & 11 & 28 & 10 & 45.4411 & 0.0876972 & 742.855 & 1105.78 & $-5.32423^{\prime} 10^{-7}$ & 0.00597666 & -1.09587 \\
\hline $\mathrm{s}$ & 11 & 28 & 11 & 113.189 & 0.112633 & 487.263 & 1132.19 & $6.94943^{\prime} 10^{-6}$ & 0.0137582 & 2.84364 \\
\hline s & 11 & 28 & 12 & 239.938 & 0.237418 & 96.0202 & 1199.97 & $-1.57956^{\prime} 10^{-6}$ & 0.0210374 & 1.98817 \\
\hline $\mathrm{s}$ & 11 & 28 & 13 & 124.679 & 0.048985 & 509.015 & 1169.79 & -0.0000225611 & 0.010991 & -1.8872 \\
\hline S & 11 & 28 & 14 & 158.357 & 0.170297 & 176.834 & 1100 . & $-6.27633^{\prime} 10^{-6}$ & 0.00900877 & 0.136975 \\
\hline $\mathrm{s}$ & 11 & 28 & 15 & 46.9284 & 0.0165948 & 825.442 & 1317. & $-2.00976^{\prime} 10^{-6}$ & 0.00338167 & 2.81191 \\
\hline s & 11 & 28 & 16 & 115.114 & 0.143905 & 258.796 & 1000 . & $-4.82969^{\prime} 10^{-8}$ & 0.00729006 & -1.74371 \\
\hline S & 11 & 28 & 17 & 121.489 & 0.326923 & 255.579 & 989.41 & 0.0000388897 & 0.00377777 & 2.94896 \\
\hline s & 11 & 28 & 18 & 116.855 & 0.302639 & 258.158 & 1000 . & 0 . & 0.00354546 & -0.389147 \\
\hline s & 11 & 28 & 19 & 65.4377 & 0.272966 & 282.949 & 979.572 & $7.68932^{\prime} 10^{-6}$ & 0.00353191 & -2.9591 \\
\hline & 11 & 28 & 20 & 135.491 & 0.519833 & 260.519 & 1000 . & $2.0703331718727345^{\prime} 10^{-6}$ & 0.00500111 & -0.965637 \\
\hline
\end{tabular}

\section{Sample 11 Scan 29:}

\begin{tabular}{|c|c|c|c|c|c|c|c|c|c|c|}
\hline s & & $\mathrm{T}$ & M & LL & w & s & $\mathrm{t}$ & a & 응 erg & $f$ \\
\hline 5 & 11 & 29 & 1 & 695.38 & 0.0235143 & 235.343 & 1043.13 & 0.0000651608 & 0.501115 & -0.374502 \\
\hline S & 11 & 29 & 2 & 129.742 & 0.00697267 & 543.309 & 1581.54 & $-2.3914166208617766^{\prime} 10^{-6}$ & 0.0606482 & -0.395312 \\
\hline$S$ & 11 & 29 & 3 & 286.324 & 0.0917758 & 70.068 & 843.42 & 0.000116956 & 0.108977 & 2.33344 \\
\hline $\mathrm{s}$ & 11 & 29 & 4 & 766.218 & 0.182946 & 47.3495 & 918.373 & -0.000023274 & 0.128352 & -2.22867 \\
\hline 5 & 11 & 29 & 5 & 114.56 & 0.0992662 & 202.654 & 1000 . & $3.1358618929066816^{\prime} 10^{-6}$ & 0.0163914 & -2.27502 \\
\hline 5 & 11 & 29 & 6 & 97.0919 & 0.0595034 & 168.829 & 572.265 & $1.3578242903836436^{\prime} 10^{-6}$ & 0.0170224 & 1.66994 \\
\hline S & 11 & 29 & 7 & 55.4974 & 0.0875297 & 903.245 & 1338.07 & $-4.5002694794232977^{\prime} 10^{-7}$ & 0.00901291 & -2.41601 \\
\hline S & 11 & 29 & 8 & 112.622 & 0.196699 & 763.391 & 1298.56 & $8.89309^{\prime} 10^{-6}$ & 0.0168222 & 0.640312 \\
\hline $\mathrm{s}$ & 11 & 29 & 9 & 127.314 & 0.111431 & 672.494 & 1243.74 & $7.00493^{\prime} 10^{-6}$ & 0.0168694 & 2.86718 \\
\hline$S$ & 11 & 29 & 10 & 164.937 & 0.223396 & 49.1378 & 1199.91 & $-1.8969552795985112^{\prime} 10^{-6}$ & 0.0168278 & 1.93822 \\
\hline S & 11 & 29 & 11 & 73.9385 & 0.0229833 & 552.782 & 903.892 & $7.75766^{\prime} 10^{-6}$ & 0.00765579 & -1.47671 \\
\hline 5 & 11 & 29 & 12 & 46.2958 & 0.190932 & 665.509 & 1209.29 & $6.42044^{\prime} 10^{-6}$ & 0.00451329 & -2.76113 \\
\hline$S$ & 11 & 29 & 13 & 118.997 & 0.168639 & 409.614 & 989.64 & -0.0000120044 & 0.0106859 & 0.055128 \\
\hline$S$ & 11 & 29 & 14 & 116.082 & 0.0476463 & 505.531 & 1159.48 & -0.0000187036 & 0.0092679 & -1.3329 \\
\hline$S$ & 11 & 29 & 15 & 74.1606 & 0.21748 & 203.607 & 800.001 & $3.16381^{\prime} 10^{-6}$ & 0.00283749 & 3.07291 \\
\hline $\mathrm{s}$ & 11 & 29 & 16 & 60.6818 & 0.327529 & 273.268 & 990.581 & 0.0000401598 & 0.00425571 & 2.56126 \\
\hline S & 11 & 29 & 17 & 104.118 & 0.30317 & 239.757 & 1000 . & $-7.41783^{\prime} 10^{-7}$ & 0.00381444 & -0.409518 \\
\hline 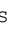 & 11 & 29 & 18 & 69.7209 & 0.273407 & 277.765 & 1000 . & $2.7729267250989928^{\prime} 10^{-6}$ & 0.00433363 & -2.19111 \\
\hline S & 11 & 29 & 19 & 60.209 & 0.118037 & 688.718 & 1219.15 & 0.0000370565 & 0.00350907 & 2.45147 \\
\hline & 11 & 29 & 20 & 44.6618 & 0.206797 & 563.845 & 1238.73 & $-6.0963997791752265^{\prime} 10^{-6}$ & 0.00246981 & 3.11169 \\
\hline
\end{tabular}


Sample 11 Scan 30:

\begin{tabular}{|c|c|c|c|c|c|c|c|c|c|c|}
\hline $\mathrm{s}$ & $\mathrm{s}$ & 1 & M & LL & w & $\mathrm{s}$ & $l$ & a & $\div$ erg & I \\
\hline & 11 & 30 & 1 & 96.625 & .0265987 & 222.907 & 023.3 & 0.0000638111 & 0.501736 & 0.117883 \\
\hline $\mathrm{S}$ & 11 & 30 & 2 & 137.279 & 0.00698941 & 514.754 & 1581.22 & $-1.9107248662711327^{\prime} 10^{-6}$ & 0.0638218 & -0.438162 \\
\hline S & 11 & 30 & 3 & 288.656 & 0.093112 & 69.487 & 838.663 & 0.000062469 & 0.108694 & 2.8507 \\
\hline $\mathrm{s}$ & 11 & 30 & 4 & 776.223 & 0.183699 & 49.4111 & 910.094 & -0.00004049 & 0.119895 & -0.672604 \\
\hline $\mathrm{s}$ & 11 & 30 & 5 & 109.491 & 0.0976072 & 250.047 & 1000 . & $-2.02232^{\prime} 10^{-9}$ & 0.014314 & -2.46874 \\
\hline S & 11 & 30 & 6 & 96.8916 & 0.0594996 & 153.416 & 557.023 & $-4.44655^{\prime} 10^{-6}$ & 0.0175978 & 2.60376 \\
\hline $\mathrm{s}$ & 11 & 30 & 7 & 66.3146 & 0.0997721 & 80.9683 & 1676.85 & -0.0000427507 & 0.0110906 & $-0.076860^{\circ}$ \\
\hline $\mathrm{S}$ & 11 & 30 & 8 & 122.14 & 0.113453 & 197.77 & 1000 . & $1.0413055593822616^{\prime} 10^{-6}$ & 0.0145783 & -0.976555 \\
\hline $\mathrm{s}$ & 11 & 30 & 9 & 138.479 & 0.194331 & 355.197 & 1420.38 & 0.0000125192 & 0.0190539 & 1.79862 \\
\hline S & 11 & 30 & 10 & 66.0564 & 0.0256778 & 676.371 & 935.525 & $-2.0289952657166607^{\prime} 10^{-6}$ & 0.00820034 & -2.01844 \\
\hline S & 11 & 30 & 11 & 52.575 & 0.0878882 & 576.198 & 1027.85 & $-1.7533872271608754^{\prime} 10^{-6}$ & 0.00615043 & -0.355017 \\
\hline S & 11 & 30 & 12 & 09.433 & 0.170629 & 31.261 & 1070.37 & -0.0000115341 & 0.0118103 & -1.05236 \\
\hline$S$ & 11 & 30 & 13 & 112.803 & 0.0446935 & 448.46 & 1094.07 & -0.0000147503 & 0.0108941 & 1.40602 \\
\hline $\mathrm{s}$ & 11 & 30 & 14 & 46.5551 & 0.0163971 & 750.636 & 1280.12 & $-2.9217703253202843^{\prime} 10^{-6}$ & 0.00414993 & -2.70131 \\
\hline S & 11 & 30 & 15 & 237.553 & 0.238696 & 85.3387 & 1200.01 & $3.1360938060868168^{\prime} 10^{-6}$ & 0.0159058 & 1.97917 \\
\hline S & 11 & 30 & 1 & 6266 & 0.32 & 44 & 7.73 & 0.0000398749 & 0.00524956 & -0.166248 \\
\hline S & 11 & 30 & 1 & .4114 & 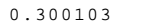 & & 7.394 & $-0.00-3 y$ & 0.00413787 & 2.1974 \\
\hline $\mathrm{s}$ & 11 & 30 & 18 & 139.197 & 0.143865 & 147.464 & 900. & 0 . & 0.00661847 & -0.213204 \\
\hline $\mathrm{s}$ & 11 & 30 & 19 & 86.6977 & 0.273125 & 319.996 & 988.146 & $2.7134587812993103^{\prime} 10^{-6}$ & 0.00458158 & 1.16865 \\
\hline & 11 & 0 & 20 & 5223 & .113886 & 7.12 & 00.12 & $4.03099^{\prime} 10^{-7}$ & 0.00248747 & -1.73944 \\
\hline
\end{tabular}

\section{Sample 11 Scan 31:}

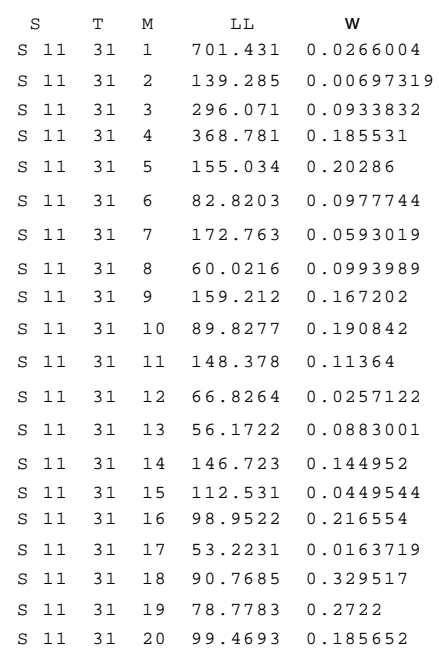

Sample 11 Scan 32:

\begin{tabular}{|c|c|c|c|c|}
\hline $\mathrm{s}$ & $\mathrm{t}$ & a & $\%$ erg & I \\
\hline 22.96 & 1023.28 & 0.0000638736 & 0.504125 & 0.11609 \\
\hline 516.798 & 1580.75 & $-1.8480176212523275^{\prime} 10^{-6}$ & 0.0643796 & -0.442332 \\
\hline 69.0739 & 838.745 & 0.0000626298 & 0.110338 & 2.85146 \\
\hline 251.933 & 993.413 & -0.0000358673 & 0.054876 & 3.12005 \\
\hline 271.933 & 1038.35 & $-7.5527662696554335^{\prime} 10^{-6}$ & 0.0380685 & -1.75878 \\
\hline 205.907 & 1000. & $3.1359^{\prime} 10^{-6}$ & 0.0145094 & -2.46701 \\
\hline 139.345 & 544.617 & $-2.23485^{\prime} 10^{-6}$ & 0.0177565 & -2.93925 \\
\hline 91.7391 & 1682.48 & $-7.23177^{\prime} 10^{-6}$ & 0.0113605 & -0.672096 \\
\hline 251.522 & 1073.43 & -0.0000248298 & 0.0266265 & -1.82404 \\
\hline 205.138 & 1500.1 & $5.27336^{\prime} 10^{-7}$ & 0.0134902 & -0.862143 \\
\hline 139.196 & 1000 . & $5.0745606928608165^{\prime} 10^{-6}$ & 0.0138906 & -0.818671 \\
\hline 678.93 & 936.604 & $-1.97078^{\prime} 10^{-6}$ & 0.00838052 & -2.056 \\
\hline 512.374 & 1043.27 & $-1.7263796215747705^{\prime} 10^{-6}$ & 0.00662384 & -1.83182 \\
\hline 170.477 & 999.878 & $-2.74034^{\prime} 10^{-6}$ & 0.0155022 & -1.4746 \\
\hline 451.964 & 1100.37 & -0.0000155602 & 0.0105512 & 1.15052 \\
\hline 479.285 & 838.983 & -0.000028657 & 0.00834604 & 2.55252 \\
\hline 758.63 & 1278.85 & $-2.90717^{\prime} 10^{-6}$ & 0.00415896 & -2.69461 \\
\hline 198.922 & 953.663 & 0.0000437715 & 0.00526535 & 2.09881 \\
\hline 35.175 & 1000. & $3.74105^{\prime} 10^{-6}$ & 0.00475713 & -1.93624 \\
\hline 29.466 & 910.76 & 0.00001365 & 0.00376696 & -0.597481 \\
\hline
\end{tabular}

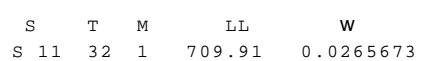

$\begin{array}{llllll}\mathrm{S} & 11 & 32 & 2 & 142.876 & 0.00698029\end{array}$ $\begin{array}{llllll}\mathrm{S} & 11 & 32 & 3 & 304.693 & 0.0932954\end{array}$ $\begin{array}{lllllll}\mathrm{S} & 11 & 32 & 4 & 478.781 & 0.177869\end{array}$

$\begin{array}{llllll}S & 11 & 32 & 5 & 86.7504 & 0.0977079\end{array}$

$\begin{array}{llllll}S & 11 & 32 & 6 & 80.695 & 0.0600991\end{array}$

$\begin{array}{llllll}\mathrm{S} & 11 & 32 & 7 & 190.092 & 0.205043\end{array}$

$\begin{array}{llllll}\mathrm{S} & 11 & 32 & 8 & 69.324 & 0.192838\end{array}$

$\begin{array}{llllll}\mathrm{S} & 11 & 32 & 9 & 300.867 & 0.157449\end{array}$

$\begin{array}{llllll}S & 11 & 32 & 10 & 80.4107 & 0.0996354\end{array}$

$\begin{array}{llllll}S & 11 & 32 & 11 & 64.3503 & 0.025722\end{array}$

$\begin{array}{llllll}\mathrm{S} & 11 & 32 & 12 & 132.085 & 0.113759\end{array}$

$\begin{array}{llllll}S & 11 & 32 & 13 & 63.3474 & 0.0880685\end{array}$

$\begin{array}{llllll}\mathrm{S} & 11 & 32 & 14 & 57.0947 & 0.16372\end{array}$

$\begin{array}{lllllll}\mathrm{S} & 11 & 32 & 15 & 42.3217 & 0.0163284\end{array}$

$\begin{array}{llllll}S & 11 & 32 & 16 & 116.276 & 0.0445329\end{array}$

$\begin{array}{lllllll}\mathrm{S} & 11 & 32 & 17 & 180.713 & 0.232938\end{array}$

$\begin{array}{llllll}\mathrm{S} & 11 & 32 & 18 & 163.298 & 0.140023\end{array}$

$\begin{array}{llllll}\mathrm{S} & 11 & 32 & 19 & 111.738 & 0.184263\end{array}$

$\begin{array}{llllll}\mathrm{S} & 11 & 32 & 20 & 88.379 & 0.329533\end{array}$

\begin{tabular}{|c|c|c|c|c|}
\hline s & $\mathrm{t}$ & a & $\div$ erg & $f$ \\
\hline 23.041 & 1023.34 & 0.0000638389 & 0.508312 & 0.110306 \\
\hline 18.12 & 1579.03 & $-1.8968635941420676^{\prime} 10^{-6}$ & 0.0653641 & -0.430067 \\
\hline 554 & 8.585 & 0.0000640405 & .111728 & 2.85019 \\
\hline 21.123 & 70.446 & -0.0000366871 & 0.0629115 & 0.261606 \\
\hline 31.656 & 1000 . & $-1.9432505282643046^{\prime} 10^{-} 6$ & 0.0144294 & -2.44032 \\
\hline 54.793 & 562.281 & $-4.6838952442113826^{\prime} 10^{-7}$ & 0.0183164 & 2.26378 \\
\hline 49.915 & 1000 . & $-4.12572^{\prime} 10^{-8}$ & 0.0224311 & -0.5986 \\
\hline 94.404 & 1187.42 & $-4.00918^{\prime} 10^{-6}$ & 0.0130985 & -2.98003 \\
\hline 22.928 & 000 . & $-7.34408^{\prime} 10^{-7}$ & 0.0340647 & -2.25141 \\
\hline 7.7034 & 1680.57 & -0.0000215422 & 0.0114606 & -0.464631 \\
\hline 81.387 & 940.18 & $-1.9197369458332857^{\prime} 10^{-6}$ & 0.00853502 & -2.15742 \\
\hline 45.747 & 1000.02 & $1.1861726698429825^{\prime} 10^{-6}$ & 0.0125559 & -0.798885 \\
\hline 14.322 & 1019.02 & $-2.1557058312015193^{\prime} 10^{-6}$ & 0.00711152 & 0.385528 \\
\hline 32.986 & 925.592 & $-4.21939^{\prime} 10^{-6}$ & 0.00603479 & 2.03061 \\
\hline 51.436 & 1270.51 & -2.9089029357989 & 0.00425613 & -2.55341 \\
\hline 59.136 & 1087.08 & -0.0000148203 & 0.0108073 & 1.69686 \\
\hline 9.4654 & 1200. & $-4.4865^{\prime} 10^{-6}$ & 0.0118866 & 2.05784 \\
\hline 21.974 & 1000 . & $-1.98585^{\prime} 10^{-6}$ & 0.00827938 & -0.6883 \\
\hline 52.476 & 1589.84 & $-8.4879^{\prime} 10^{-6}$ & 0.00713406 & 1.04537 \\
\hline 36.62 & 945.172 & 0.0000437566 & 0.00502909 & -1.44809 \\
\hline
\end{tabular}


Sample 11 Scan 33:

\begin{tabular}{|c|c|c|c|c|c|c|c|c|c|c|}
\hline $\mathrm{s}$ & & $\mathrm{T}$ & M & LL & w & $\mathrm{s}$ & $t$ & a & $\div$ erg & $f$ \\
\hline $\mathrm{s}$ & 11 & 33 & 1 & 721.483 & 0.0265426 & 222.717 & 1023.59 & 0.0000639627 & 0.513969 & 0.10236 \\
\hline $\mathrm{s}$ & 11 & 33 & 2 & 147.818 & 0.00696992 & 519.414 & 1575.27 & $-1.9323395724103926^{\prime} 10^{-6}$ & 0.0666855 & -0.408236 \\
\hline $\mathrm{s}$ & 11 & 33 & 3 & 521.67 & 0.136819 & 99.41 & 871.588 & -0.000480622 & 0.170075 & -0.566824 \\
\hline $\mathrm{s}$ & 11 & 33 & 4 & 87.8148 & 0.0869891 & 676.619 & 1094.47 & $-1.2240763183469783^{\prime} 10^{-6}$ & 0.0208774 & 0.181069 \\
\hline $\mathrm{s}$ & 11 & 33 & 5 & 86.6276 & 0.192381 & 444.906 & 1202.68 & $-8.60227^{\prime} 10^{-6}$ & 0.0188747 & 0.682459 \\
\hline $\mathrm{s}$ & 11 & 33 & 6 & 204.18 & 0.110198 & 184.955 & 1100.34 & $-1.7179843308240873^{\prime} 10^{-8}$ & 0.0267185 & 0.30593 \\
\hline $\mathrm{s}$ & 11 & 33 & 7 & 138.819 & 0.0518072 & 407.492 & 723.933 & 0.0000128119 & 0.0235722 & -0.0981888 \\
\hline $\mathrm{s}$ & 11 & 33 & 8 & 184.946 & 0.133069 & 166.429 & 900. & $1.2464212829979956^{\prime} 10^{-7}$ & 0.0220196 & -1.08572 \\
\hline $\mathrm{s}$ & 11 & 33 & 9 & 151.235 & 0.172194 & 647.932 & 1137.99 & -0.0000131463 & 0.0191264 & 0.0388625 \\
\hline $\mathrm{s}$ & 11 & 33 & 10 & 81.5517 & 0.0306529 & 511.869 & 1130.3 & -0.0000991591 & 0.00917426 & 0.0193774 \\
\hline $\mathrm{s}$ & 11 & 33 & 11 & 44.555 & 0.0153068 & 934.814 & 969.814 & $-4.5215823435929824^{\prime} 10$ & 0.00470503 & 1.44973 \\
\hline s & 11 & 33 & 12 & 100.778 & 0.0720455 & 241.973 & 906.734 & -0.0000125314 & 0.00986817 & -2.4378 \\
\hline s & 11 & 33 & 13 & 106.392 & 0.226184 & 376.83 & 1103.42 & -0.0000126431 & 0.00942633 & -1.21377 \\
\hline s & 11 & 33 & 14 & 191.668 & 0.246899 & 220.198 & 1098.22 & $-4.85002^{\prime} 10^{-6}$ & 0.0117245 & 2.09436 \\
\hline$S$ & 11 & 33 & 15 & 127.73 & 0.110986 & 74.9146 & 1686.28 & -0.000132596 & 0.0086629 & -0.70377 \\
\hline s & 11 & 33 & 16 & 64.2494 & 0.042913 & 1075.82 & 1451.39 & $1.6806512125798446^{\prime} 10^{-7}$ & 0.00395668 & 2.18949 \\
\hline s & 11 & 33 & 17 & 106.967 & 0.329557 & 223.349 & 942.623 & 0.0000430132 & 0.00404651 & -0.561545 \\
\hline $\mathrm{s}$ & 11 & 33 & 18 & 70.0793 & 0.174435 & 437.12 & 865.808 & -0.000011769 & 0.00376158 & 1.14862 \\
\hline S & 11 & 33 & 19 & 63.2442 & 0.0647956 & 163.346 & 1387.31 & $-2.25806^{\prime} 10^{-6}$ & 0.00316737 & -0.402838 \\
\hline $\mathrm{s}$ & 11 & 33 & 20 & 45.95 & 0.107232 & 286.733 & 683.555 & 0.0000223833 & 0.00218488 & -0.250053 \\
\hline
\end{tabular}

\section{Sample 11 Scan 34:}

\begin{tabular}{llllll}
$S$ & $S$ & $M$ & \multicolumn{1}{c}{ LL } & \multicolumn{1}{c}{$W$} \\
$S$ & 11 & 34 & 1 & 722.062 & 0.0265391 \\
$S$ & 11 & 34 & 2 & 148.218 & 0.00696207 \\
$S$ & 11 & 34 & 3 & 525.345 & 0.136894 \\
$S$ & 11 & 34 & 4 & 87.3816 & 0.0869565 \\
$S$ & 11 & 34 & 5 & 197.781 & 0.110071 \\
$S$ & 11 & 34 & 6 & 97.1974 & 0.189125 \\
$S$ & 11 & 34 & 7 & 139.565 & 0.0518777 \\
$S$ & 11 & 34 & 8 & 174.311 & 0.132833 \\
$S$ & 11 & 34 & 9 & 102.609 & 0.16849 \\
$S$ & 11 & 34 & 10 & 76.1533 & 0.0257525 \\
$S$ & 11 & 34 & 11 & 43.6994 & 0.0153074 \\
$S$ & 11 & 34 & 12 & 92.1772 & 0.0716626 \\
$S$ & 11 & 34 & 13 & 101.955 & 0.225852 \\
$S$ & 11 & 34 & 14 & 133.695 & 0.24757 \\
$S$ & 11 & 34 & 15 & 96.9169 & 0.187741 \\
$S$ & 11 & 34 & 16 & 128.405 & 0.11153 \\
$S$ & 11 & 34 & 17 & 65.8705 & 0.0428534 \\
$S$ & 11 & 34 & 18 & 138.365 & 0.315463 \\
$S$ & 11 & 34 & 19 & 61.2697 & 0.0651591 \\
$S$ & 11 & 34 & 20 & 43.091 & 0.107307
\end{tabular}

\begin{tabular}{|c|c|c|}
\hline $\mathrm{s}$ & $\mathrm{t}$ & a \\
\hline 223.287 & 1023.17 & 0.000064137 \\
\hline 520.668 & 1574.68 & $-1.8897225537085075^{\prime} 10^{-6}$ \\
\hline 98.9912 & 871.337 & -0.000480291 \\
\hline 678.058 & 1089.28 & $-1.18931^{\prime} 10^{-6}$ \\
\hline 189.287 & 1100 & $1.55037^{\prime} 10^{-6}$ \\
\hline 455.531 & 1103.75 & -0.0000198536 \\
\hline 409.959 & 722.352 & 0.0000130101 \\
\hline 153.022 & 899.996 & $3.15851^{\prime} 10^{-8}$ \\
\hline 286.398 & 987.32 & -0.0000101563 \\
\hline 535.087 & 1105.88 & -0.0000987439 \\
\hline 828.327 & 1018.25 & $-5.01328^{\prime} 10^{-7}$ \\
\hline 248.782 & 915.914 & -0.0000108635 \\
\hline 396.277 & 1091.05 & -0.0000122022 \\
\hline 249.042 & 1099.28 & $-4.86233^{\prime} 10^{-6}$ \\
\hline 212.929 & 1537.44 & $-4.83035^{\prime} 10^{-6}$ \\
\hline 75.9693 & 1687.3 & -0.000143813 \\
\hline 1084.4 & 1463.32 & $1.8291874529894744^{\prime} 10^{-7}$ \\
\hline 174.703 & 1100 & $1.1436478291201147^{\prime} 10^{-6}$ \\
\hline 166.424 & 1391.34 & $-2.5231306364643216^{\prime} 10^{-6}$ \\
\hline 278.25 & 688.066 & 0.000023017 \\
\hline
\end{tabular}

\begin{tabular}{ll}
\multicolumn{1}{c}{ o. erg } & \multicolumn{1}{c}{$f$} \\
0.514251 & 0.0966749 \\
0.0668121 & -0.414413 \\
0.170816 & -0.577022 \\
0.020682 & 0.591129 \\
0.0276807 & 0.326265 \\
0.0184149 & 1.20521 \\
0.0234997 & -0.0418248 \\
0.0211282 & -1.11606 \\
0.0132373 & 0.325982 \\
0.00898499 & 0.686417 \\
0.00485521 & 0.720768 \\
0.00955988 & 3.13426 \\
0.00960906 & 1.49522 \\
0.0111722 & 1.72109 \\
0.00723731 & -1.79297 \\
0.00856958 & -0.813471 \\
0.00398715 & 1.6699 \\
0.00537763 & -0.873427 \\
0.00315764 & -0.713725 \\
0.00210973 & -0.750001
\end{tabular}

Sample 11 Scan 35:

\begin{tabular}{|c|c|c|c|c|c|c|c|c|c|}
\hline $\mathrm{s}$ & $\mathrm{T}$ & M & LL & w & $\mathrm{s}$ & $\mathrm{t}$ & a & $\div$ erg & $f$ \\
\hline S 11 & 35 & 1 & 714.119 & 0.0265879 & 223.038 & 1022.99 & 0.0000640576 & 0.510377 & 0.105743 \\
\hline 11 & 35 & 2 & 146.769 & 0.0069986 & 514.469 & 1578.37 & $-1.8434701318674945^{\prime} 10^{-6}$ & 0.0667342 & -0.443933 \\
\hline S 11 & 35 & 3 & 389.059 & 0.0871052 & 152.565 & 808.338 & -0.000394492 & 0.135991 & 0.0259997 \\
\hline S 11 & 35 & 4 & 249.848 & 0.148489 & 140.07 & 961.768 & 0.000283787 & 0.0630297 & -3.08669 \\
\hline 11 & 35 & 5 & 95.2651 & 0.0868781 & 600.243 & 1111.94 & $6.05881^{\prime} 10^{-7}$ & 0.0201787 & -1.91264 \\
\hline S 11 & 35 & 6 & 161.318 & 0.0542112 & 331.718 & 800.988 & -0.0000158918 & 0.0300769 & -2.66821 \\
\hline S 11 & 35 & 7 & 101.234 & 0.196086 & 410.551 & 1386.07 & 0.0000160126 & 0.0165417 & 2.1342 \\
\hline 11 & 35 & 8 & 73.3635 & 0.0136908 & 887.104 & 916.38 & $-5.24435^{\prime} 10^{-6}$ & 0.0109833 & -2.81163 \\
\hline s 11 & 35 & 9 & 102.599 & 0.108525 & 607.127 & 1110.97 & $-4.45252^{\prime} 10^{-6}$ & 0.0140634 & -0.678242 \\
\hline 11 & 35 & 10 & 99.8527 & 0.0679404 & 402.937 & 707.496 & -0.0000120661 & 0.012369 & -1.27415 \\
\hline 11 & 35 & 11 & 104.741 & 0.230985 & 57.7407 & 1200 . & $1.1975243775424786^{\prime} 10^{-6}$ & 0.0110398 & 1.82061 \\
\hline 11 & 35 & 12 & 79.9342 & 0.0997022 & 263.088 & 1659.36 & $8.91698^{\prime} 10^{-7}$ & 0.00820094 & 1.81628 \\
\hline S 11 & 35 & 13 & 103.178 & 0.168036 & 475.498 & 989.973 & -0.000015005 & 0.00966125 & 0.35997 \\
\hline S 11 & 35 & 14 & 74.3126 & 0.0556212 & 149.505 & 1400. & $3.13609^{\prime} 10^{-6}$ & 0.0059953 & -1.41517 \\
\hline S 11 & 35 & 15 & 50.3798 & 0.0257313 & 157.195 & 1222.48 & 0.000106561 & 0.00407272 & 2.59527 \\
\hline S 11 & 35 & 16 & 166.075 & 0.315584 & 157.615 & 1100. & $1.39486^{\prime} 10^{-6}$ & 0.00813651 & -0.803417 \\
\hline S 11 & 35 & 17 & 128.445 & 0.273305 & 202.594 & 1000 . & $3.10776^{\prime} 10^{-6}$ & 0.00580361 & -2.33054 \\
\hline S 11 & 35 & 。 & 105.065 & 0.189332 & 30 & 916.653 & 0.0000174169 & 0.00646898 & -2.61049 \\
\hline S 11 & 35 & 19 & 64.7745 & 0.0207554 & 299.697 & 529.296 & 0.0000495524 & 0.00366251 & 1.95121 \\
\hline 11 & 35 & 20 & 63.9271 & 0.299715 & 467.287 & 904.233 & $-9.96518^{\prime} 10^{-6}$ & 0.00338014 & -2.50179 \\
\hline
\end{tabular}


Sample 11 Scan 36:

\begin{tabular}{|c|c|c|c|c|c|c|c|c|c|c|}
\hline $\mathrm{s}$ & & $\mathrm{T}$ & M & LL & w & $\mathrm{s}$ & $\mathrm{t}$ & a & $\frac{\circ}{\partial}$ erg & $f$ \\
\hline $\mathrm{s}$ & 11 & 36 & 1 & 716.607 & 0.0265744 & 223.215 & 1023.19 & 0.0000640822 & 0.511593 & 0.103455 \\
\hline $\mathrm{s}$ & 11 & 36 & 2 & 149.005 & 0.00701889 & 511.347 & 1579.35 & $-1.86153^{\prime} 10^{-6}$ & 0.0675084 & -0.451186 \\
\hline $\mathrm{s}$ & 11 & 36 & 3 & 320.876 & 0.0939401 & 67.9488 & 837.617 & 0.0000474029 & 0.115266 & 2.95548 \\
\hline $\mathrm{s}$ & 11 & 36 & 4 & 564.769 & 0.182487 & 60.5713 & 900.409 & -0.0000144811 & 0.106386 & 1.06353 \\
\hline $\mathrm{s}$ & 11 & 36 & 5 & 98.0974 & 0.0975777 & 232.513 & 1000.01 & $1.2207755367319256^{\prime} 10^{-6}$ & 0.0131788 & -2.51302 \\
\hline $\mathrm{s}$ & 11 & 36 & 6 & 105.573 & 0.0579071 & 145.088 & 558.466 & $-5.13192^{\prime} 10^{-7}$ & 0.0184147 & 2.50076 \\
\hline $\mathrm{s}$ & 11 & 36 & 7 & 74.9528 & 0.0981132 & 103.829 & 1690.18 & $9.96514^{\prime} 10^{-6}$ & 0.0120369 & -1.43008 \\
\hline $\mathrm{s}$ & 11 & 36 & 8 & 59.0538 & 0.0257733 & 704.354 & 951.634 & $-1.88915^{\prime} 10^{-6}$ & 0.0088682 & -2.45397 \\
\hline $\mathrm{s}$ & 11 & 36 & 9 & 107.104 & 0.113343 & 282.807 & 1077.39 & $6.31375^{\prime} 10^{-6}$ & 0.0148066 & 2.9223 \\
\hline $\mathrm{s}$ & 11 & 36 & 10 & 45.9625 & 0.0879341 & 564.663 & 1013.98 & $-2.0951526983891363^{\prime} 10^{-6}$ & 0.00588364 & 0.80924 \\
\hline $\mathrm{s}$ & 11 & 36 & 11 & 124.754 & 0.195104 & 332.536 & 1414.45 & 0.0000163752 & 0.0146713 & 2.93066 \\
\hline $\mathrm{s}$ & 11 & 36 & 12 & 107.566 & 0.0461881 & 462.204 & 1128.4 & -0.0000156368 & 0.0112608 & -0.119712 \\
\hline $\mathrm{s}$ & 11 & 36 & 13 & 166.279 & 0.144478 & 165.375 & 900. & $2.9450267148168505^{\prime} 10^{-6}$ & 0.0123966 & -0.0193639 \\
\hline $\mathrm{s}$ & 11 & 36 & 14 & 52.8053 & 0.0163624 & 755.313 & 1273.88 & $-2.98217^{\prime} 10^{-6}$ & 0.00446262 & -2.61309 \\
\hline $\mathrm{s}$ & 11 & 36 & 15 & 161.088 & 0.235906 & 59.716 & 1200. & $1.1747013680521735^{\prime} 10^{-6}$ & 0.0116941 & 1.79111 \\
\hline S & 11 & 36 & 16 & 173.495 & 0.31616 & 158.439 & 1100.08 & $2.0397709396104935^{\prime} 10^{-7}$ & 0.00768742 & -0.820864 \\
\hline $\mathrm{s}$ & 11 & 36 & 17 & 127.895 & 0.17649 & 198.71 & 1200. & $-1.26052^{\prime} 10^{-6}$ & 0.00707961 & 1.83287 \\
\hline s & 11 & 36 & 18 & 111.056 & 0.274436 & 252.435 & 1000.31 & 0 . & 0.00453267 & -2.14486 \\
\hline $\mathrm{s}$ & 11 & 36 & 19 & 69.7896 & 0.298856 & 430.788 & 879.022 & -0.0000110754 & 0.00345849 & -1.13901 \\
\hline $\mathrm{s}$ & 11 & 36 & 20 & 33.2366 & 0.134726 & 88.2713 & 1803.41 & 0.000114476 & 0.00156413 & 2.21572 \\
\hline
\end{tabular}

\section{Sample 11 Scan 37:}

\begin{tabular}{|c|c|c|c|c|c|c|c|c|c|c|}
\hline $\mathrm{s}$ & & $\mathrm{T}$ & M & LL & w & $\mathrm{s}$ & $\mathrm{t}$ & a & $\frac{\circ}{6}$ erg & $f$ \\
\hline s & 11 & 37 & 1 & 722.614 & 0.0265213 & 223.653 & 1023.25 & 0.0000640806 & 0.514518 & 0.0955883 \\
\hline $\mathrm{s}$ & 11 & 37 & 2 & 151.914 & 0.00699128 & 515.281 & 1576.3 & $-1.8891478129560046^{\prime} 10^{-6}$ & 0.068316 & -0.42933 \\
\hline s & 11 & 37 & 3 & 199.806 & 0.104036 & 257.53 & 952.067 & -0.0000253055 & 0.0753015 & -2.35047 \\
\hline s & 11 & 37 & 4 & 131.633 & 0.0862638 & 259.793 & 1011.85 & $-3.6306634326940763^{\prime} 10^{-6}$ & 0.0368874 & 1.11435 \\
\hline $\mathrm{s}$ & 11 & 37 & 5 & 451.93 & 0.178792 & 112.249 & 888.603 & -0.0000212685 & 0.0783321 & -3.12904 \\
\hline $\mathrm{s}$ & 11 & 37 & 6 & 176.141 & 0.0673663 & 405.344 & 761.386 & -0.0000149254 & 0.0364477 & 2.12012 \\
\hline $\mathrm{s}$ & 11 & 37 & 7 & 110.314 & 0.194034 & 823.565 & 1457.76 & 0.0000163893 & 0.019765 & 0.768996 \\
\hline s & 11 & 37 & 8 & 280.404 & 0.144864 & 115.827 & 899.934 & $2.0014153373665129^{\prime} 10^{-7}$ & 0.0324164 & 0.360306 \\
\hline $\mathrm{s}$ & 11 & 37 & 9 & 69.3961 & 0.0258239 & 704.981 & 954.269 & $-1.8316396499319585^{\prime} 10^{-6}$ & 0.00918845 & -2.53569 \\
\hline $\mathrm{s}$ & 11 & 37 & 10 & 93.7619 & 0.114667 & 575.279 & 930.987 & -0.0000117828 & 0.0114439 & -1.64168 \\
\hline $\mathrm{s}$ & 11 & 37 & 11 & 82.2307 & 0.174166 & 248.384 & 1143.29 & $-7.76571 \cdot 10^{-6}$ & 0.00918979 & -0.790473 \\
\hline s & 11 & 37 & 12 & 106.813 & 0.0462758 & 463.209 & 1134.12 & -0.0000183354 & 0.0108632 & -0.242434 \\
\hline s & 11 & 37 & 13 & 48.1211 & 0.0163164 & 739.485 & 1267.52 & $-2.98699^{\prime} 10^{-6}$ & 0.00452749 & -2.51455 \\
\hline s & 11 & 37 & 14 & 137.544 & 0.233418 & 83.1665 & 1199.77 & $-3.13609^{\prime} 10^{-6}$ & 0.0110082 & 1.84511 \\
\hline $\mathrm{s}$ & 11 & 37 & 15 & 145.857 & 0.316202 & 188.487 & 1100. & $4.15504^{\prime} 10^{-6}$ & 0.00782968 & -0.884831 \\
\hline $\mathrm{s}$ & 11 & 37 & 16 & 197.274 & 0.0993348 & 129.578 & 1679.71 & 0.0000287147 & 0.0130699 & -0.490075 \\
\hline $\mathrm{s}$ & 11 & 37 & 17 & 101.494 & 0.192514 & 199.603 & 775.146 & -0.0000167047 & 0.00487065 & 1.11266 \\
\hline s & 11 & 37 & 18 & 64.5871 & 0.299192 & 449.082 & 877.915 & -0.000010806 & 0.00344384 & -0.967731 \\
\hline s & 11 & 37 & 19 & 50.7779 & 0.21909 & 294.717 & 1002.58 & 0.0000434813 & 0.00255557 & -2.62126 \\
\hline s & 11 & 37 & 20 & 118.1 & 0.273527 & 212.961 & 1000. & $2.15635^{\prime} 10^{-6}$ & 0.00449724 & -2.05321 \\
\hline
\end{tabular}

\section{Sample 11 Scan 38:}

\begin{tabular}{|c|c|c|c|c|c|c|c|c|c|}
\hline $\mathrm{s}$ & $\mathrm{T}$ & M & LL & w & $\mathrm{s}$ & $\mathrm{t}$ & a & $\therefore$ erg & $f$ \\
\hline S 11 & 38 & 1 & 734.293 & 0.0228507 & 237.419 & 1046.88 & 0.0000654992 & 0.520155 & -0.483392 \\
\hline S 11 & 38 & 2 & 147.035 & 0.00697561 & 547.525 & 1576.18 & $-2.27256^{\prime} 10^{-6}$ & 0.0655505 & -0.390026 \\
\hline S 11 & 38 & 3 & 203.2 & 0.102822 & 247.493 & 935.019 & -0.000028336 & 0.0756322 & -0.536349 \\
\hline S 11 & 38 & 4 & 262.214 & 0.179347 & 171.815 & 894.373 & -0.0000232313 & 0.06587 & 2.01549 \\
\hline S 11 & 38 & 5 & 261.773 & 0.0866107 & 229.109 & 1002.63 & -0.0000311422 & 0.0487039 & 2.34531 \\
\hline S 11 & 38 & 6 & 88.3158 & 0.06393 & 366.519 & 668.619 & -0.0000144832 & 0.0188982 & 1.96904 \\
\hline S 11 & 38 & 7 & 108.33 & 0.193976 & 837.442 & 1471.94 & 0.0000156514 & 0.0210136 & -1.91721 \\
\hline S 11 & 38 & 8 & 263.016 & 0.146484 & 115.197 & 899.997 & $-1.1978812205940977^{\prime} 10^{-6}$ & 0.0342846 & 0.429088 \\
\hline S 11 & 38 & 9 & 89.0013 & 0.103182 & 354.346 & 1625.3 & 0.0000256976 & 0.0127202 & -1.27784 \\
\hline S 11 & 38 & 10 & 82.7555 & 0.171931 & 251.688 & 1132.09 & -0.0000102966 & 0.0108524 & 0.851518 \\
\hline S 11 & 38 & 11 & 66.4946 & 0.0486024 & 592.914 & 1055.6 & $1.1236737449130198^{\prime} 10^{-6}$ & 0.0080932 & 2.96969 \\
\hline S 11 & 38 & 12 & 74.3595 & 0.0246585 & 362.391 & 880.373 & 0.0000139261 & 0.00843536 & -1.20205 \\
\hline S 11 & 38 & 13 & 100.595 & 0.195076 & 200.455 & 800 . & 0 . & 0.00939297 & 1.91435 \\
\hline S 11 & 38 & 14 & 107.627 & 0.111615 & 338.596 & 810.635 & -0.0000137166 & 0.0101916 & -0.494165 \\
\hline S 11 & 38 & 15 & 141.015 & 0.233978 & 84.5543 & 1200 . & $1.2391385542960395^{\prime} 10^{-6}$ & 0.0116448 & 1.68512 \\
\hline S 11 & 38 & 16 & 135.071 & 0.316169 & 163.253 & 1100.13 & $3.95042^{\prime} 10^{-6}$ & 0.00763503 & -1.00046 \\
\hline S 11 & 38 & 17 & 70.7625 & 0.0271689 & 380.03 & 1476.1 & 0.0000174204 & 0.00481019 & 1.09713 \\
\hline S 11 & 38 & 18 & 71.5706 & 0.0613349 & 254.916 & 1354.22 & $3.6626518917024992^{\prime} 10^{-6}$ & 0.00453071 & 1.24455 \\
\hline S 11 & 38 & 19 & 71.4457 & 0.0835189 & 269.914 & 1533.35 & $3.38778^{\prime} 10^{-7}$ & 0.00421068 & -0.543305 \\
\hline S 11 & 38 & 20 & 119.959 & 0.273526 & 220.492 & 999.998 & $3.1348^{\prime} 10^{-6}$ & 0.00507335 & -2.12473 \\
\hline
\end{tabular}


Sample 11 Scan 39:

\begin{tabular}{|c|c|c|c|c|c|c|c|c|c|c|}
\hline $\mathrm{s}$ & & $\mathrm{T}$ & M & LL & w & $\mathrm{s}$ & $\mathrm{t}$ & a & $\therefore$ erg & $f$ \\
\hline $\mathrm{s}$ & 11 & 39 & 1 & 735.261 & 0.0228147 & 237.696 & 1047.1 & 0.0000654842 & 0.52062 & -0.489041 \\
\hline $\mathrm{s}$ & 11 & 39 & 2 & 149.097 & 0.00698697 & 547.597 & 1577.87 & $-2.2553609403381866^{\prime} 10^{-6}$ & 0.0663379 & -0.404361 \\
\hline $\mathrm{s}$ & 11 & 39 & 3 & 334.608 & 0.0932744 & 67.4782 & 841.424 & 0.000104332 & 0.117313 & 2.47604 \\
\hline $\mathrm{s}$ & 11 & 39 & 4 & 612.903 & 0.181517 & 52.366 & 903.186 & -0.0000332612 & 0.102375 & 0.429584 \\
\hline $\mathrm{s}$ & 11 & 39 & 5 & 105.875 & 0.0984786 & 221.467 & 1000 . & $3.0563844370792317^{\prime} 10^{-6}$ & 0.0137757 & -2.46154 \\
\hline $\mathrm{s}$ & 11 & 39 & 6 & 113.97 & 0.0597087 & 150.745 & 560.079 & $1.3747^{\prime} 10^{-6}$ & 0.0192695 & 2.38769 \\
\hline $\mathrm{s}$ & 11 & 39 & 7 & 116.849 & 0.099742 & 976.482 & 1667.63 & -0.000176485 & 0.0176216 & 1.13971 \\
\hline $\mathrm{s}$ & 11 & 39 & 8 & 56.8721 & 0.178783 & 542.604 & 1256.4 & $-6.41278^{\prime} 10^{-7}$ & 0.00785851 & -1.95275 \\
\hline $\mathrm{s}$ & 11 & 39 & 9 & 107.722 & 0.195435 & 303.197 & 1337.15 & $4.12653^{\prime} 10^{-6}$ & 0.0137137 & -0.576224 \\
\hline $\mathrm{s}$ & 11 & 39 & 10 & 76.8216 & 0.0236912 & 562.162 & 901.191 & $6.60753^{\prime} 10^{-6}$ & 0.00891576 & -1.46701 \\
\hline $\mathrm{s}$ & 11 & 39 & 11 & 128.063 & 0.111396 & 637.654 & 1239.85 & $7.72622^{\prime} 10^{-6}$ & 0.0134215 & -2.89854 \\
\hline $\mathrm{s}$ & 11 & 39 & 12 & 50.2308 & 0.087725 & 1331.55 & 1457.4 & $-5.94398^{\prime} 10^{-7}$ & 0.00481258 & -0.323895 \\
\hline $\mathrm{s}$ & 11 & 39 & 13 & 124.947 & 0.316509 & 158.301 & 1100.13 & $5.0542^{\prime} 10^{-6}$ & 0.00837977 & -0.977433 \\
\hline $\mathrm{S}$ & 11 & 39 & 14 & 180.437 & 0.238023 & 83.242 & 1199.73 & $4.16871^{\prime} 10^{-6}$ & 0.0137432 & 1.71841 \\
\hline $\mathrm{s}$ & 11 & 39 & 15 & 115.905 & 0.0459762 & 585.672 & 1089.75 & -0.0000199251 & 0.00780307 & 1.76255 \\
\hline $\mathrm{s}$ & 11 & 39 & 16 & 57.7547 & 0.168283 & 605.879 & 1058.27 & $-3.3202180595181825^{\prime} 10^{-6}$ & 0.00356335 & 0.941468 \\
\hline s & 11 & 39 & 17 & 47.869 & 0.103496 & 757.316 & 926.915 & $2.0291207686787996^{\prime} 10^{-6}$ & 0.00280137 & 0.874241 \\
\hline $\mathrm{s}$ & 11 & 39 & 18 & 144.887 & 0.147176 & 193.595 & 900. & $-9.2254^{\prime} 10^{-8}$ & 0.00556229 & -0.419107 \\
\hline $\mathrm{s}$ & 11 & 39 & 19 & 62.9748 & 0.299707 & 428.605 & 909.698 & -0.0000101433 & 0.00314745 & 2.07232 \\
\hline $\mathrm{s}$ & 11 & 39 & 20 & 156.335 & 0.274708 & 194.959 & 1000. & $3.2157139578611764^{\prime} 10^{-6}$ & 0.00513769 & -2.22754 \\
\hline
\end{tabular}

\section{Sample 11 Scan 40:}

\begin{tabular}{|c|c|c|c|c|c|}
\hline & $\mathrm{s}$ & $\mathrm{T}$ & M & LL & w \\
\hline $\mathrm{s}$ & 11 & 40 & 1 & 743.156 & 0.0227492 \\
\hline $\mathrm{s}$ & 11 & 40 & 2 & 151.908 & 0.00701497 \\
\hline $\mathrm{s}$ & 11 & 40 & 3 & 344.2 & 0.0931881 \\
\hline $\mathrm{s}$ & 11 & 40 & 4 & 383.844 & 0.177396 \\
\hline S & 11 & 40 & 5 & 90.9292 & 0.0984896 \\
\hline $\mathrm{s}$ & 11 & 40 & 6 & 95.1807 & 0.0596981 \\
\hline $\mathrm{s}$ & 11 & 40 & 7 & 66.0648 & 0.0980296 \\
\hline $\mathrm{s}$ & 11 & 40 & 8 & 135.913 & 0.202715 \\
\hline $\mathrm{s}$ & 11 & 40 & 9 & 336.228 & 0.147796 \\
\hline $\mathrm{s}$ & 11 & 40 & 10 & 48.2912 & 0.0881454 \\
\hline $\mathrm{s}$ & 11 & 40 & 11 & 93.1354 & 0.11339 \\
\hline $\mathrm{s}$ & 11 & 40 & 12 & 77.7034 & 0.0232375 \\
\hline $\mathrm{s}$ & 11 & 40 & 13 & 129.814 & 0.19114 \\
\hline $\mathrm{s}$ & 11 & 40 & 14 & 103.262 & 0.0464756 \\
\hline $\mathrm{s}$ & 11 & 40 & 15 & 136.81 & 0.233868 \\
\hline $\mathrm{s}$ & 11 & 40 & 16 & 162.251 & 0.316465 \\
\hline $\mathrm{s}$ & 11 & 40 & 17 & 129.816 & 0.27342 \\
\hline $\mathrm{s}$ & 11 & 40 & 18 & 113.786 & 0.169089 \\
\hline $\mathrm{s}$ & 11 & 40 & 19 & 64.7044 & 0.301859 \\
\hline S & 11 & 40 & 20 & 87.5094 & 0.0245995 \\
\hline
\end{tabular}

\begin{tabular}{|c|c|c|c|c|}
\hline $\mathrm{s}$ & $\mathrm{t}$ & a & $\frac{\circ}{\partial}$ erg & $f$ \\
\hline 237.726 & 1047.78 & 0.0000654649 & 0.52439 & -0.498808 \\
\hline 544.58 & 1577.72 & $-2.37197^{\prime} 10^{-6}$ & 0.0669643 & -0.399862 \\
\hline 67.8402 & 841.584 & 0.0000968823 & 0.11885 & 2.4856 \\
\hline 147.34 & 910.293 & -0.0000314099 & 0.0645616 & -0.730786 \\
\hline 233.86 & 1000 . & $1.8327616993474376^{\prime} 10^{-6}$ & 0.0141 & -2.41997 \\
\hline 155.028 & 557.164 & $-2.5204122434616836^{\prime} 10^{-7}$ & 0.0191211 & 2.57498 \\
\hline 88.2656 & 1666.64 & -0.0000147398 & 0.0121717 & 0.914021 \\
\hline 552.947 & 1189.26 & 0.0000151015 & 0.0227878 & -2.31591 \\
\hline 177.421 & 900.012 & $-2.25473^{\prime} 10^{-6}$ & 0.0242415 & 0.400857 \\
\hline 667.909 & 1064.64 & $-1.3697942763373743^{\prime} 10^{-6}$ & 0.00623649 & 2.58218 \\
\hline 370.82 & 1092.74 & $7.20405^{\prime} 10^{-6}$ & 0.0112096 & 1.11177 \\
\hline 542.809 & 890.753 & $8.12779^{\prime} 10^{-6}$ & 0.00858563 & -1.20338 \\
\hline 263.675 & 1001.1 & 0.000035694 & 0.0126619 & -0.218015 \\
\hline 452.277 & 1127.47 & -0.0000177112 & 0.00918236 & 0.146461 \\
\hline 96.301 & 1200 . & $1.1987926845788253^{\prime} 10^{-6}$ & 0.0105039 & 1.73752 \\
\hline 164.932 & 1100 . & $6.2730469969219315^{\prime} 10^{-6}$ & 0.00797825 & -0.893067 \\
\hline 209.322 & 1000.24 & $5.07431^{\prime} 10^{-6}$ & 0.00633485 & -2.1152 \\
\hline 227.687 & 1000 . & $-1.93273^{\prime} 10^{-6}$ & 0.00596223 & -1.85234 \\
\hline 623.649 & 1020.96 & $-8.367211^{\prime} 10^{-6}$ & 0.00336032 & -0.0505713 \\
\hline 301.666 & 1551.34 & 0.0000116818 & 0.00421222 & -0.67508 \\
\hline
\end{tabular}

Sample 11 Scan 41:

\begin{tabular}{llllll}
$S$ & T & M & \multicolumn{1}{c}{ LL } & \multicolumn{1}{c}{ W } \\
$S$ & 11 & 41 & 1 & 738.553 & 0.0227003 \\
$S$ & 11 & 41 & 2 & 151.311 & 0.00703514 \\
$S$ & 11 & 41 & 3 & 497.711 & 0.0860382 \\
$S$ & 11 & 41 & 4 & 126.483 & 0.108026 \\
$S$ & 11 & 41 & 5 & 457.737 & 0.179589 \\
$S$ & 11 & 41 & 6 & 139.33 & 0.0748274 \\
$S$ & 11 & 41 & 7 & 48.0921 & 0.0484642 \\
$S$ & 11 & 41 & 8 & 118.538 & 0.205062 \\
$S$ & 11 & 41 & 9 & 274.526 & 0.158488 \\
$S$ & 11 & 41 & 10 & 87.4357 & 0.0860317 \\
$S$ & 11 & 41 & 11 & 157.775 & 0.13816 \\
$S$ & 11 & 41 & 12 & 73.3711 & 0.0232293 \\
$S$ & 11 & 41 & 13 & 81.0056 & 0.192975 \\
$S$ & 11 & 41 & 14 & 42.2026 & 0.037838 \\
$S$ & 11 & 41 & 15 & 79.0094 & 0.0784388 \\
$S$ & 11 & 41 & 16 & 141.96 & 0.316725 \\
$S$ & 11 & 41 & 17 & 114.027 & 0.234711 \\
$S$ & 11 & 41 & 18 & 61.7578 & 0.21793 \\
$S$ & 11 & 41 & 19 & 144.897 & 0.273203 \\
$S$ & 11 & 41 & 20 & 62.155 & 0.125678
\end{tabular}

\begin{tabular}{|c|c|c|c|c|}
\hline s & $\mathrm{t}$ & a & $\%$ erg & $f$ \\
\hline 238.332 & 1047.92 & 0.0000655406 & 0.522195 & -0.507191 \\
\hline 540.487 & 1584.09 & $-2.33662534770440306^{\prime} 10^{-6}$ & 0.0670293 & -0.445904 \\
\hline 97.7406 & 800.269 & $-2.2472943482185547^{\prime} 10^{-6}$ & 0.0843622 & -0.0157728 \\
\hline 447.406 & 977.087 & $-2.8502464562622107^{\prime} 10^{-6}$ & 0.0387169 & 0.914867 \\
\hline 224.662 & 889.965 & -0.000026556 & 0.056735 & 2.84455 \\
\hline 646.46 & 847.306 & -0.0000221509 & 0.0299681 & 1.51711 \\
\hline 597.19 & 1030. & $6.95322^{\prime} 10^{-7}$ & 0.00941194 & -2.0641 \\
\hline 265.04 & 999.989 & -0.0000235572 & 0.0211779 & -0.311181 \\
\hline 137.043 & 1000 & $-1.81554^{\prime} 10^{-6}$ & 0.0282701 & -2.2988 \\
\hline 486.213 & 1572.81 & -0.0000402228 & 0.0118542 & -2.97351 \\
\hline 208.325 & 1000 & $-1.93821 \cdot 10^{-6}$ & 0.0127767 & -0.744701 \\
\hline 639.685 & 885.137 & $7.34767^{\prime} 10^{-6}$ & 0.00827416 & -0.982874 \\
\hline 207.692 & 1490.32 & 0.0000218074 & 0.0084569 & 0.770335 \\
\hline 3208.66 & 827.943 & $6.52213^{\prime} 10^{-6}$ & 0.00414185 & -1.44517 \\
\hline 387.25 & 701.648 & -0.0000233087 & 0.00729943 & -1.84633 \\
\hline 189.31 & 1100 & $-1.6591929722383039^{\prime} 10^{-6}$ & 0.00832571 & -0.873611 \\
\hline 138.402 & 1200 & $3.1105953673363565^{\prime} 10^{-6}$ & 0.00799381 & 1.86612 \\
\hline 384.36 & 963.031 & $-5.3007^{\prime} 10^{-6}$ & 0.00433992 & -0.249251 \\
\hline 195.08 & 1000.01 & $5.06829^{\prime} 10^{-6}$ & 0.00735787 & -2.09383 \\
\hline 339.577 & 814.548 & $-6.70986^{\prime} 10^{-6}$ & 0.00366204 & -0.384425 \\
\hline
\end{tabular}


Sample 11 Scan 42:

\begin{tabular}{|c|c|c|c|c|c|c|c|c|c|c|}
\hline $\mathrm{s}$ & & $T$ & M & LL & w & $\mathrm{s}$ & $t$ & a & $\frac{\circ}{\partial}$ erg & $f$ \\
\hline $\mathrm{s}$ & 11 & 42 & 1 & 745.984 & 0.0224934 & 239.267 & 1049.63 & 0.000065505 & 0.525732 & -0.538678 \\
\hline $\mathrm{s}$ & 11 & 42 & 2 & 157.02 & 0.00706959 & 536.854 & 1588.61 & $-2.30437^{\prime} 10^{-6}$ & 0.0688489 & -0.48205 \\
\hline $\mathrm{s}$ & 11 & 42 & 3 & 350.595 & 0.0935283 & 67.4742 & 841.777 & 0.000100737 & 0.119727 & 2.47563 \\
\hline $\mathrm{s}$ & 11 & 42 & 4 & 329.949 & 0.176302 & 222.108 & 887.387 & -0.0000340166 & 0.0544286 & -2.90114 \\
\hline $\mathrm{s}$ & 11 & 42 & 5 & 92.0959 & 0.0986228 & 232.139 & 1000. & $5.30606^{\prime} 10^{-7}$ & 0.0139564 & -2.40078 \\
\hline $\mathrm{s}$ & 11 & 42 & 6 & 94.4063 & 0.0599504 & 156.729 & 556.757 & $7.10042^{\prime} 10^{-7}$ & 0.0195223 & 2.60785 \\
\hline $\mathrm{s}$ & 11 & 42 & 7 & 177.925 & 0.196226 & 216.824 & 871.432 & -0.0000206452 & 0.0201038 & 0.459721 \\
\hline $\mathrm{s}$ & 11 & 42 & 8 & 75.0358 & 0.0996933 & 79.5999 & 1683.97 & -0.0000296749 & 0.0127938 & -0.723678 \\
\hline $\mathrm{s}$ & 11 & 42 & 9 & 304.432 & 0.151855 & 173.659 & 900. & $-1.1269291118004927^{\prime} 10^{-6}$ & 0.0316308 & 0.579341 \\
\hline $\mathrm{s}$ & 11 & 42 & 10 & 93.9147 & 0.113545 & 363.65 & 1107.76 & $5.9336^{\prime} 10^{-6}$ & 0.0118942 & -0.494922 \\
\hline $\mathrm{s}$ & 11 & 42 & 11 & 56.9441 & 0.0879417 & 581.856 & 1011.34 & $-1.6035017419140223^{\prime} 10^{-6}$ & 0.00668636 & 1.07537 \\
\hline $\mathrm{s}$ & 11 & 42 & 12 & 125.465 & 0.0387585 & 581.353 & 928.706 & -0.0000199004 & 0.0134549 & 2.56121 \\
\hline $\mathrm{s}$ & 11 & 42 & 13 & 89.3024 & 0.176265 & 584.997 & 1271.14 & -0.0000102977 & 0.00859879 & 1.83807 \\
\hline $\mathrm{s}$ & 11 & 42 & 14 & 69.0349 & 0.0190413 & 1132.99 & 1465.71 & $-5.55016^{\prime} 10^{-6}$ & 0.00614048 & 1.02587 \\
\hline $\mathrm{s}$ & 11 & 42 & 15 & 95.5592 & 0.194554 & 212.441 & 1365.94 & $1.3624041708287266^{\prime} 10^{-6}$ & 0.00782618 & 0.252601 \\
\hline $\mathrm{s}$ & 11 & 42 & 16 & 181.386 & 0.316609 & 167.028 & 1100 . & $3.2440167545013514^{\prime} 10^{-6}$ & 0.0084669 & -0.755316 \\
\hline $\mathrm{s}$ & 11 & 42 & 17 & 136.359 & 0.233618 & 211.187 & 1200. & $-6.23796^{\prime} 10^{-9}$ & 0.00851823 & 1.86835 \\
\hline $\mathrm{s}$ & 11 & 42 & 18 & 149.927 & 0.27319 & 196.227 & 1000 . & $8.27814^{\prime} 10^{-6}$ & 0.00721011 & -2.00807 \\
\hline $\mathrm{s}$ & 11 & 42 & 19 & 86.4759 & 0.0381286 & 1005.3 & 1521.38 & -0.0000114946 & 0.00446128 & -0.932336 \\
\hline $\mathrm{s}$ & 11 & 42 & 20 & 75.2532 & 0.213207 & 247.4 & 835.291 & $9.24949^{\prime} 10^{-6}$ & 0.00358029 & 2.35541 \\
\hline
\end{tabular}

\section{Sample 11 Scan 43:}

\begin{tabular}{llllll}
$S$ & $T$ & $M$ & \multicolumn{1}{c}{$L L$} & \multicolumn{1}{c}{$W$} \\
$S$ & 11 & 43 & 1 & 985.546 & 0.0219293 \\
$S$ & 11 & 43 & 2 & 259.436 & 0.00708724 \\
$S$ & 11 & 43 & 3 & 431.258 & 0.086147 \\
$S$ & 11 & 43 & 4 & 159.562 & 0.107872 \\
$S$ & 11 & 43 & 5 & 177.792 & 0.0685343 \\
$S$ & 11 & 43 & 6 & 568.803 & 0.181141 \\
$S$ & 11 & 43 & 7 & 86.8238 & 0.0483781 \\
$S$ & 11 & 43 & 8 & 112.733 & 0.0274036 \\
$S$ & 11 & 43 & 9 & 139.399 & 0.0861201 \\
$S$ & 11 & 43 & 10 & 104.649 & 0.018572 \\
$S$ & 11 & 43 & 11 & 245.734 & 0.142162 \\
$S$ & 11 & 43 & 12 & 114.005 & 0.194468 \\
$S$ & 11 & 43 & 13 & 138.907 & 0.0788291 \\
$S$ & 11 & 43 & 14 & 82.7933 & 0.031439 \\
$S$ & 11 & 43 & 15 & 75.988 & 0.007856 \\
$S$ & 11 & 43 & 16 & 184.594 & 0.235461 \\
$S$ & 11 & 43 & 17 & 127.678 & 0.172068 \\
$S$ & 11 & 43 & 18 & 205.354 & 0.316753 \\
$S$ & 11 & 43 & 19 & 116.577 & 0.274305 \\
$S$ & 11 & 43 & 20 & 95.0224 & 0.128017
\end{tabular}

\begin{tabular}{|c|c|c|}
\hline s & $\mathrm{t}$ & a \\
\hline 240.028 & 1057.86 & 0.0000648888 \\
\hline 538.647 & 1590.57 & $-2.41939^{\prime} 10^{-6}$ \\
\hline 102.853 & 799.961 & $1.1818572839276466^{\circ} 10^{-6}$ \\
\hline 443.463 & 963.722 & $-2.81842^{\prime} 10^{-6}$ \\
\hline 495.095 & 714.677 & -0.0000225233 \\
\hline 56.7086 & 894.193 & -0.0000220683 \\
\hline 573.806 & 1056.52 & $5.40615^{\prime} 10^{-8}$ \\
\hline 227.027 & 680.115 & -0.0000211085 \\
\hline 388.59 & 1575.6 & -0.0000363647 \\
\hline 640.464 & 1421.47 & $-5.56971 \cdot 10^{-6}$ \\
\hline 140.968 & 899.999 & $-6.84966^{\prime} 10^{-7}$ \\
\hline 407.557 & 1311.58 & $4.62271^{\prime} 10^{-6}$ \\
\hline 470.334 & 709.899 & -0.0000230544 \\
\hline 266.437 & 1282.75 & -0.0000152661 \\
\hline 2282.72 & 4556.39 & $8.14902^{\prime} 10^{-6}$ \\
\hline 73.6538 & 1200. & $-2.1214605283969312^{\prime} 10^{-8}$ \\
\hline 522.981 & 1123.7 & -0.0000130815 \\
\hline 141.054 & 1100 & $-1.89594^{\prime} 10^{-6}$ \\
\hline 201.795 & 1000. & $3.75249^{\prime} 10^{-6}$ \\
\hline 69.3732 & 1700 . & $-1.93568^{\prime} 10^{-6}$ \\
\hline
\end{tabular}

\begin{tabular}{ll}
\multicolumn{1}{c}{$\frac{c}{\mathrm{O} e r g}$} & \multicolumn{1}{c}{$f$} \\
0.626765 & -0.61364 \\
0.085151 & -0.4595 \\
0.0716522 & 0.323995 \\
0.0318144 & 2.73311 \\
0.0299545 & -1.16274 \\
0.0483182 & 2.45508 \\
0.00878416 & -3.06086 \\
0.0103246 & -1.46001 \\
0.0112577 & -2.99907 \\
0.00747786 & 1.99066 \\
0.0115303 & 0.386252 \\
0.00606075 & -1.45908 \\
0.00650908 & -2.21964 \\
0.00347076 & 1.47437 \\
0.00294222 & -0.793519 \\
0.00504117 & 2.08989 \\
0.0038627 & 3.03198 \\
0.0032476 & -0.425505 \\
0.00270098 & -1.72376 \\
0.00163858 & -2.0828
\end{tabular}

\section{Sample 11 Scan 44:}

\begin{tabular}{|c|c|c|c|c|c|c|c|c|c|c|}
\hline $\mathrm{s}$ & & $\mathrm{T}$ & M & LL & w & $\mathrm{s}$ & $t$ & a & $\frac{\circ}{\partial}$ erg & $f$ \\
\hline & 11 & 44 & 1 & 1318.37 & 0.0261041 & 220.516 & 1038.69 & 0.0000602951 & 0.732428 & 0.0800918 \\
\hline & 11 & 44 & 2 & 626.604 & 0.00715781 & 472.809 & 1585.71 & $-1.4800687137687586^{\prime} 10^{-6}$ & 0.123839 & -0.438238 \\
\hline $\mathrm{s}$ & 11 & 44 & 3 & 100.91 & 0.0247449 & 94.6566 & 1165.09 & 0.0000157019 & 0.0135709 & -1.183 \\
\hline $\mathrm{s}$ & 11 & 44 & 4 & 467.609 & 0.0854007 & 118.976 & 809.562 & -0.000432205 & 0.0477329 & 0.477079 \\
\hline s & 11 & 44 & 5 & 88.3825 & 0.0870063 & 711.091 & 1117.58 & $-1.1746922219902008^{\prime} 10^{-6}$ & 0.00674329 & -1.09349 \\
\hline $\mathrm{s}$ & 11 & 44 & 6 & 295.471 & -0.0039788 & 259.988 & 705.586 & 0.000228525 & 0.0186063 & 1.56676 \\
\hline s & 11 & 44 & 7 & 132.583 & 0.0598593 & 139.943 & 1322.03 & $5.15447^{\prime} 10^{-6}$ & 0.00408428 & -2.49798 \\
\hline$s$ & 11 & 44 & 8 & 80.9025 & 0.109625 & 490.035 & 1250.92 & $1.27354^{\prime} 10^{-7}$ & 0.00386893 & 3.10035 \\
\hline s & 11 & 44 & 9 & 122.581 & 0.0495476 & 1072.59 & 1032.49 & $6.31697^{\prime} 10^{-6}$ & 0.00529689 & -2.96425 \\
\hline $\mathrm{s}$ & 11 & 44 & 10 & 102.471 & 0.0281375 & 387.306 & 695.824 & -0.0000179126 & 0.00395591 & -1.67769 \\
\hline $\mathrm{s}$ & 11 & 44 & 11 & 117.551 & 0.182696 & 442.817 & 973.681 & -0.0000239506 & 0.00406582 & 0.764646 \\
\hline $\mathrm{s}$ & 11 & 44 & 12 & 97.9554 & 0.0983936 & 557.394 & 1455.59 & $1.8298657744371995^{\circ}$ & 0.00304142 & -2.51828 \\
\hline $\mathrm{s}$ & 11 & 44 & 13 & 70.6757 & 0.0742861 & 203.289 & 1000 . & $-8.8077^{\prime} 10^{-6}$ & 0.00186308 & -1.86542 \\
\hline$S$ & 11 & 44 & 14 & 164.397 & 0.0231788 & 271.652 & 1709.31 & 0.0000162824 & 0.00419768 & 2.23685 \\
\hline $\mathrm{s}$ & 11 & 44 & 15 & 85.2869 & 0.13249 & 110.068 & 900. & $-6.9140347534115065^{\prime} 10^{-9}$ & 0.00152787 & -0.688865 \\
\hline $\mathrm{s}$ & 11 & 44 & 16 & 135.344 & 0.0667948 & 33.2886 & 444.814 & -0.000231543 & 0.00278019 & -2.62189 \\
\hline $\mathrm{s}$ & 11 & 44 & 17 & 96.6208 & 0.197224 & 227.765 & 865.445 & -0.0000190431 & 0.00163423 & 1.85822 \\
\hline $\mathrm{s}$ & 11 & 44 & 18 & 125.893 & 0.171472 & 621.863 & 1144.08 & -0.0000137914 & 0.0020743 & -0.727592 \\
\hline $\mathrm{s}$ & 11 & 44 & 19 & 68.6871 & 0.00971793 & 707.608 & 1022.63 & 0.0000123566 & 0.00102634 & 2.1779 \\
\hline & 11 & 44 & 20 & 186.381 & 0.316644 & 136.576 & 1100.13 & $1.0574099120895902^{\prime} 10^{-7}$ & 0.00142187 & -0.508529 \\
\hline
\end{tabular}


Sample 11 Scan 45:

\begin{tabular}{|c|c|c|c|c|c|c|c|c|c|c|}
\hline $\mathrm{s}$ & & 1 & M & LL & w & $\mathrm{s}$ & $\mathrm{t}$ & a & $\%$ erg & $f$ \\
\hline S & 11 & 45 & 1 & 1460.89 & 0.0194523 & 241.356 & 1087.33 & 0.0000627829 & 0.767969 & -0.937311 \\
\hline $\mathrm{s}$ & 11 & 45 & 2 & 733.897 & 0.00712431 & 541.753 & 1595.79 & $-2.62704^{\prime} 10^{-6}$ & 0.120177 & -0.412549 \\
\hline $\mathrm{s}$ & 11 & 45 & 3 & 137.05 & 0.0330973 & 528.344 & 839.255 & -0.0000244942 & 0.0141915 & 0.507944 \\
\hline $\mathrm{s}$ & 11 & 45 & 4 & 63.753 & .0873364 & 66.1623 & 30.879 & 0.0000233558 & 0.0294607 & -1.87814 \\
\hline S & 11 & 45 & 5 & 201.818 & 0.0567989 & 144.71 & 549.517 & $-7.28833^{\prime} 10^{-6}$ & 0.0122439 & -2.36335 \\
\hline $\mathrm{S}$ & 11 & 45 & 6 & 197.315 & 0.0246982 & 354.399 & 1534.54 & 0.0000104958 & 0.00983219 & 0.162477 \\
\hline s & 11 & 45 & 7 & 88.7271 & 0.0176936 & 252.837 & 1125.49 & $6.74409^{\prime} 10^{-6}$ & 0.00382699 & 0.485188 \\
\hline s & 11 & 45 & 8 & 779.324 & 0.176284 & 47.8445 & 891.619 & -0.0000247051 & 0.0115626 & 2.8756 \\
\hline $\mathrm{s}$ & 11 & 45 & 9 & 100.296 & 0.097848 & 767.655 & 1317.71 & $-1.8863454849167582^{\prime} 10^{-6}$ & 0.00283276 & -1.27438 \\
\hline $\mathrm{s}$ & 11 & 45 & 10 & 154.257 & 0.0434836 & 799.347 & 1625.97 & $3.0816057759729623^{\prime} 10^{-6}$ & 0.00383836 & 1.58927 \\
\hline S & 11 & 45 & 11 & 103.043 & 0.0879408 & 583.55 & 1373.34 & $7.72286^{\prime} 10^{-7}$ & 0.00225309 & 1.86616 \\
\hline $\mathrm{S}$ & 11 & 45 & 12 & 145.234 & 0.111165 & 380.807 & 1183.64 & $6.63658^{\prime} 10^{-6}$ & 0.0028061 & -2.20978 \\
\hline $\mathrm{s}$ & 11 & 45 & 13 & 98.6825 & 0.193784 & 339.655 & 1244.48 & $-5.28675^{\prime} 10^{-6}$ & 0.00168696 & -0.73549 \\
\hline $\mathrm{s}$ & 11 & 45 & 14 & 94.2973 & 0.00874313 & 819.548 & 199.588 & -0.0000360584 & 0.00146367 & -2.81883 \\
\hline S & 11 & 45 & 15 & 108.365 & 0.0599589 & 586.647 & 1345.04 & $5.29201^{\prime} 10^{-6}$ & 0.00151225 & 3.13145 \\
\hline $\mathrm{s}$ & 11 & 45 & 16 & 173.435 & 0.143585 & 156.191 & 900. & $5.07431^{\prime} 10^{-6}$ & 0.00161701 & -0.0540 \\
\hline $\mathrm{s}$ & 11 & 45 & 17 & 60.6671 & 0.0771093 & 868.674 & 1417.98 & $-3.84909^{\prime} 10^{-6}$ & 0.000682564 & -3.06537 \\
\hline s & 11 & 45 & 18 & .488 & 0.235276 & 78.8583 & 1200 . & $1.5473924158736936^{\prime} 10^{-6}$ & 0.00141987 & 2.08311 \\
\hline $\mathrm{s}$ & 11 & 45 & 19 & 229.791 & 0.316737 & 146.167 & 1100 . & $1.2131393732153834^{\prime} 10^{-6}$ & 0.00108014 & -0.432094 \\
\hline 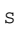 & 11 & 45 & 20 & 24.783 & 0.274362 & 199.921 & 1000.31 & $3.1163967730816747^{\prime} 10^{-6}$ & 0.0009307 & -1.81931 \\
\hline
\end{tabular}

\section{Sample 11 Scan 46:}

\begin{tabular}{llllll}
$S$ & $T$ & $M$ & \multicolumn{1}{c}{ LL } & \multicolumn{1}{c}{ W } \\
S 11 & 46 & 1 & 1458.6 & 0.0204601 \\
$S$ & 11 & 46 & 2 & 301.433 & 0.00805971 \\
$S$ & 11 & 46 & 3 & 521.525 & 0.0489051 \\
$S$ & 11 & 46 & 4 & 231.655 & 0.0320151 \\
$S$ & 11 & 46 & 5 & 389.225 & 0.0887553 \\
$S$ & 11 & 46 & 6 & 139.903 & 0.022043 \\
$S$ & 11 & 46 & 7 & 487.314 & 0.181083 \\
$S$ & 11 & 46 & 8 & 80.3953 & 0.0977301 \\
$S$ & 11 & 46 & 9 & 145.398 & 0.0598147 \\
$S$ & 11 & 46 & 10 & 120.12 & 0.0327424 \\
$S$ & 11 & 46 & 11 & 90.7379 & 0.000363583 \\
$S$ & 11 & 46 & 12 & 121.344 & 0.0885528 \\
$S$ & 11 & 46 & 13 & 66.3093 & 0.0449246 \\
$S$ & 11 & 46 & 14 & 102.179 & 0.199377 \\
$S$ & 11 & 46 & 15 & 87.7716 & 0.0836984 \\
$S$ & 11 & 46 & 16 & 248.576 & 0.145833 \\
$S$ & 11 & 46 & 17 & 97.1823 & 0.109395 \\
$S$ & 11 & 46 & 18 & 55.7139 & 0.0563697 \\
$S$ & 11 & 46 & 19 & 125.793 & 0.176125 \\
$S$ & 11 & 46 & 20 & 230.034 & 0.316667
\end{tabular}

\begin{tabular}{|c|c|c|}
\hline $\mathrm{s}$ & $t$ & a \\
\hline 337.941 & 1145.82 & 0.000028236 \\
\hline 396.88 & 2260.38 & $2.30419^{\prime} 10^{-6}$ \\
\hline 314.047 & 822.648 & $3.4039928710220665^{\prime} 10^{-6}$ \\
\hline 439.936 & 963.476 & $-4.22094^{\prime} 10^{-6}$ \\
\hline 66.5632 & 840.801 & 0.0000995547 \\
\hline 636.668 & 1460.75 & $-9.31911^{\prime} 10^{-7}$ \\
\hline 87.7807 & 893.736 & -0.0000171481 \\
\hline 249.868 & 999.972 & $3.8298198142160266^{\prime} 10^{-8}$ \\
\hline 1345.57 & 1338.17 & $5.41906^{\prime} 10^{-6}$ \\
\hline 24.2508 & 1697.92 & -0.000630126 \\
\hline 298.425 & 1093.5 & $9.12603^{\prime} 10^{-8}$ \\
\hline 351.451 & 739.037 & -0.0000512738 \\
\hline 917.277 & 1379.69 & $3.4849648323234904^{\prime} 10^{-6}$ \\
\hline 501.251 & 1272.02 & 0.0000171244 \\
\hline 470.521 & 1385.79 & 0.0000186643 \\
\hline 154.798 & 900. & $-1.2041383118079767^{\prime} 10^{-6}$ \\
\hline 464.987 & 1387.19 & 0.0000104378 \\
\hline 1024.71 & 1065.61 & $2.44827^{\prime} 10^{-6}$ \\
\hline 208.653 & 1199.91 & $3.21889^{\prime} 10^{-6}$ \\
\hline 8.859 & 1100 . & $2.49113^{\prime} 10^{-8}$ \\
\hline
\end{tabular}

$\frac{\circ}{6}$ erg 0.767439 0.0605204

0.0696464 0.0210366 0.0260183 0.00713876 0.0114022

0.00264517

0.00453195 0.00327765 0.00222704 0.0026674 0.0013265 0.00187937 0.00146794

0.00259852 0.00124122 0.000659037 0.00105095 0.00116197

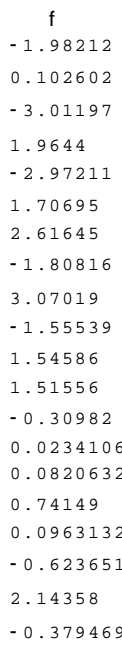

\section{Sample 11 Scan 47:}

\begin{tabular}{llllll}
$S$ & $T$ & $M$ & LL & \multicolumn{1}{c}{$W$} \\
$S$ & 11 & 47 & 1 & 1292.36 & 0.0261575 \\
$S$ & 11 & 47 & 2 & 597.577 & 0.00718629 \\
$S$ & 11 & 47 & 3 & 427.106 & 0.102835 \\
$S$ & 11 & 47 & 4 & 179.311 & 0.00712505 \\
$S$ & 11 & 47 & 5 & 236.021 & 0.0588374 \\
$S$ & 11 & 47 & 6 & 119.828 & 0.0872155 \\
$S$ & 11 & 47 & 7 & 173.425 & 0.0476831 \\
$S$ & 11 & 47 & 8 & 169.753 & 0.110939 \\
$S$ & 11 & 47 & 9 & 109.815 & 0.0806622 \\
$S$ & 11 & 47 & 10 & 123.783 & 0.0247669 \\
$S$ & 11 & 47 & 11 & 111.656 & 0.17991 \\
$S$ & 11 & 47 & 12 & 357.68 & 0.131306 \\
$S$ & 11 & 47 & 13 & 148.051 & 0.0541855 \\
$S$ & 11 & 47 & 14 & 82.3381 & 0.0583867 \\
$S$ & 11 & 47 & 15 & 52.8973 & 0.190366 \\
$S$ & 11 & 47 & 16 & 27.9586 & 0.0214092 \\
$S$ & 11 & 47 & 17 & 74.9642 & 0.207507 \\
$S$ & 11 & 47 & 18 & 74.4274 & 0.0753166 \\
$S$ & 11 & 47 & 19 & 156.296 & 0.31696 \\
$S$ & 11 & 47 & 20 & 92.6554 & 0.127613
\end{tabular}

\begin{tabular}{|c|c|c|c|c|}
\hline s & $\mathrm{t}$ & a & $\frac{\circ}{\partial}$ erg & $f$ \\
\hline .098 & 1037.6 & 0.0000607096 & 0.725379 & 0.0829885 \\
\hline .588 & 1586.15 & $-1.4467416926655304^{\prime} 10^{-6}$ & 0.12285 & -0.457059 \\
\hline .6148 & 835.348 & -0.000476731 & 0.0519672 & -1.91386 \\
\hline 1.942 & 977.515 & -0.0000344826 & 0.0159968 & 1.68961 \\
\hline 4.973 & 564.835 & $6.22792^{\prime} 10^{-6}$ & 0.017089 & 2.792 \\
\hline 9.635 & 1169.8 & $-5.3622^{\prime} 10^{-7}$ & 0.00724948 & 0.618324 \\
\hline 0.987 & 1239.42 & -0.0000581989 & 0.00906702 & 2.1404 \\
\hline .598 & 1099.78 & $-1.6077751730202666^{\prime} 10^{-8}$ & 0.00621916 & 1.07582 \\
\hline .027 & 1191.4 & -0.0000242853 & 0.00433266 & 2.31312 \\
\hline 5.364 & 1723.79 & -0.0000284023 & 0.00434597 & 2.3709 \\
\hline .003 & 917.588 & -0.0000336387 & 0.0034841 & -1.2369 \\
\hline 3.848 & 900.019 & $1.9914010644829957^{\prime} 10^{-6}$ & 0.00562948 & -0.694493 \\
\hline .4521 & 936.177 & 0.0000714317 & 0.0032841 & 2.40907 \\
\hline 59.39 & 1707.46 & $5.86573^{\prime} 10^{-6}$ & 0.00162667 & 0.0170028 \\
\hline .794 & 1364.09 & $2.6393266377721796^{\prime} 10^{-6}$ & 0.000976533 & 0.605006 \\
\hline 3.06 & 2945.1 & $-8.44028^{\prime} 10^{-6}$ & 0.000495649 & -1.98141 \\
\hline .716 & 952.613 & 0.0000205077 & 0.00125958 & 2.6579 \\
\hline 2.74 & 1482.48 & $4.3866638830371155^{\prime} 10^{-6}$ & 0.00116054 & 2.43213 \\
\hline 683 & 1100 & $3.2427960596234064^{\prime} 10^{-6}$ & 0.00136839 & -0.446962 \\
\hline .33 & 1943.13 & -0.000024097 & 0.00120805 & -0.86397 \\
\hline
\end{tabular}


Sample 11 Scan 48:

\begin{tabular}{|c|c|c|c|c|c|c|c|c|c|c|}
\hline s & & $\mathrm{T}$ & M & LL & w & $\mathrm{s}$ & $t$ & a & $\frac{\circ}{8}$ erg & $f$ \\
\hline 3 & 11 & 48 & 1 & 1099.11 & 0.0213107 & 240.702 & 1064.76 & 0.0000643691 & 0.666832 & -0.696288 \\
\hline$s$ & 11 & 48 & 2 & 336.496 & 0.00712319 & 534.623 & 1589.57 & $-2.46076^{\prime} 10^{-6}$ & 0.0950044 & -0.445444 \\
\hline $\mathrm{s}$ & 11 & 48 & 3 & 474.294 & 0.0908591 & 72.3927 & 845.298 & 0.0000970347 & 0.089649 & 2.6346 \\
\hline 5 & 11 & 48 & 4 & 149.27 & 0.0586849 & 151.925 & 562.23 & $-3.95208^{\prime} 10^{-6}$ & 0.0204678 & 2.67112 \\
\hline S & 11 & 48 & 5 & 89.5645 & 0.0982904 & 213.589 & 1000 . & $-7.0781^{\prime} 10^{-7}$ & 0.0100354 & -1.86906 \\
\hline $\mathrm{s}$ & 11 & 48 & 6 & 77.0378 & 0.0885515 & 87.4853 & 1679.17 & $-7.91645^{\prime} 10^{-6}$ & 0.00868573 & 0.228937 \\
\hline$S$ & 11 & 48 & 7 & 147.302 & 0.0386383 & 525.589 & 933.176 & -0.0000196264 & 0.0148653 & 2.63332 \\
\hline $\mathrm{s}$ & 11 & 48 & 8 & 502.742 & 0.185625 & 84.7869 & 889.02 & -0.000038033 & 0.0250026 & -2.64773 \\
\hline $\mathrm{s}$ & 11 & 48 & 9 & 101.193 & 0.0326417 & 893.938 & 1441.35 & -0.0000679132 & 0.00660831 & 2.59739 \\
\hline S & 11 & 48 & 10 & 125.446 & 0.112305 & 560.889 & 1173.43 & $6.5633412251783735^{\prime} 10^{-6}$ & 0.00729668 & -1.21516 \\
\hline S & 11 & 48 & 11 & 306.096 & 0.14726 & 128.237 & 899.917 & $-9.25058^{\prime} 10^{-8}$ & 0.0109046 & 0.95012 \\
\hline 5 & 11 & 48 & 12 & 166.809 & 0.0204105 & 320.308 & 1591.03 & -0.0000207109 & 0.00671237 & -1.00314 \\
\hline S & 11 & 48 & 13 & 103.673 & 0.0881708 & 487.234 & 1026.58 & $-3.61217^{\prime} 10^{-6}$ & 0.00363835 & 0.247064 \\
\hline$S$ & 11 & 48 & 14 & 133.121 & 0.19481 & 385.976 & 1237.23 & $7.29683^{\prime} 10^{-7}$ & 0.00415138 & 0.851106 \\
\hline S & 11 & 48 & 15 & 73.3011 & 0.0254822 & 468.705 & 941.623 & $-7.71384^{\prime} 10^{-7}$ & 0.00206067 & -1.93708 \\
\hline 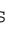 & 11 & 48 & 16 & 73.7643 & 0.0442013 & 1022.38 & 1551.02 & $1.4005692063530654^{\prime} 10^{-6}$ & 0.00192668 & -1.60196 \\
\hline 5 & 11 & 48 & 17 & 92.7485 & 0.168046 & 229.192 & 1100. & $-1.9448479257040374^{\prime} 10^{-6}$ & 0.00159319 & 0.725551 \\
\hline $\mathrm{s}$ & 11 & 48 & 18 & 146.368 & 0.0690528 & 216.514 & 1371.74 & -0.0000253132 & 0.00320988 & 0.774075 \\
\hline$S$ & 11 & 48 & 19 & 73.1397 & 0.181316 & 1007.94 & 1382.06 & $-4.08654^{\prime} 10^{-6}$ & 0.00143629 & 1.66426 \\
\hline$S$ & 11 & 48 & 20 & 208.219 & 0.316886 & 149.822 & 1100 & $6.2678^{\prime} 10^{-6}$ & 0.00191023 & -0.353354 \\
\hline
\end{tabular}

\section{Sample 11 Scan 49:}

\begin{tabular}{llllll}
$S$ & $T$ & $M$ & \multicolumn{1}{c}{ LL } & \multicolumn{1}{c}{$W$} \\
$S$ & 11 & 49 & 1 & 932.722 & 0.0263339 \\
$S$ & 11 & 49 & 2 & 263.46 & 0.00717442 \\
$S$ & 11 & 49 & 3 & 484.926 & 0.0867459 \\
$S$ & 11 & 49 & 4 & 215.3 & 0.0519884 \\
$S$ & 11 & 49 & 5 & 131.834 & 0.086798 \\
$S$ & 11 & 49 & 6 & 299.852 & 0.108875 \\
$S$ & 11 & 49 & 7 & 69.7135 & 0.0154613 \\
$S$ & 11 & 49 & 8 & 124.039 & 0.179151 \\
$S$ & 11 & 49 & 9 & 121.079 & 0.101339 \\
$S$ & 11 & 49 & 10 & 121.489 & 0.0266806 \\
$S$ & 11 & 49 & 11 & 303.454 & 0.132413 \\
$S$ & 11 & 49 & 12 & 142.066 & 0.0725421 \\
$S$ & 11 & 49 & 13 & 147.127 & 0.0581466 \\
$S$ & 11 & 49 & 14 & 86.4503 & 0.192646 \\
$S$ & 11 & 49 & 15 & 117.467 & 0.246277 \\
$S$ & 11 & 49 & 16 & 68.7092 & 0.221638 \\
$S$ & 11 & 49 & 17 & 143.029 & 0.174479 \\
$S$ & 11 & 49 & 18 & 145.849 & 0.317074 \\
$S$ & 11 & 49 & 19 & 65.6999 & 0.0593223 \\
$S$ & 11 & 49 & 20 & 59.6972 & 0.297484
\end{tabular}

\begin{tabular}{|c|c|c|c|c|}
\hline s & $\mathrm{t}$ & a & $\therefore$ erg & $f$ \\
\hline 123 & 1027.91 & 0.0000638791 & 0.606519 & 0.0757232 \\
\hline 302 & 1584.69 & $-1.6531736594478307^{\prime} 10^{-6}$ & 0.0909112 & -0.497083 \\
\hline .129 & 825.496 & -0.000530788 & 0.115742 & -1.32101 \\
\hline .739 & 714.514 & $7.91022^{\prime} 10^{-6}$ & 0.0359182 & 0.859166 \\
\hline .018 & 1096.87 & $-9.98621 \cdot 10^{-7}$ & 0.0184669 & 0.159086 \\
\hline .871 & 1100 & $1.7683970019501512^{\prime} 10^{-9}$ & 0.0200455 & 0.58045 \\
\hline .067 & 1156.64 & $-2.5182259040249284^{\prime} 10^{-6}$ & 0.00747467 & -0.967795 \\
\hline .408 & 911.975 & -0.0000342904 & 0.0120767 & -0.363992 \\
\hline .114 & 1619.66 & $-3.61822^{\prime} 10^{-7}$ & 0.0104285 & -0.0210632 \\
\hline .007 & 1086.42 & $-2.4325262605686826^{\prime} 10^{-6}$ & 0.00926867 & 0.694302 \\
\hline .614 & 900.034 & $-1.6554311323758265^{\prime} 10^{-6}$ & 0.0110378 & -0.549488 \\
\hline 089 & 930.021 & $-6.64885^{\prime} 10^{-6}$ & 0.00804053 & 1.92393 \\
\hline .211 & 1351.93 & 0.0000183344 & 0.00720411 & 1.37911 \\
\hline .779 & 1453.6 & $-1.1689289975205547^{\prime} 10^{-6}$ & 0.00261329 & 2.29701 \\
\hline .704 & 1100. & 0.0000204879 & 0.00455736 & 1.36393 \\
\hline 628 & 922.171 & -0.0000102084 & 0.00254302 & 1.84696 \\
\hline 109 & 1200. & $-2.6562151193154315^{\prime} 10^{-9}$ & 0.0040349 & 2.01033 \\
\hline 71 & 1100 & $3.87643^{\prime} 10^{-6}$ & 0.00302306 & -0.345802 \\
\hline 188 & 2187.37 & -0.0000176402 & 0.00182475 & -1.07178 \\
\hline 696 & 819.313 & -0.0000129307 & 0.00155719 & -1.69469 \\
\hline
\end{tabular}

\section{Sample 11 Scan 50:}

\begin{tabular}{llllll}
$S$ & $T$ & $M$ & \multicolumn{1}{c}{ LL } & \multicolumn{1}{c}{$W$} \\
$S$ & 11 & 50 & 1 & 778.079 & 0.021914 \\
$S$ & 11 & 50 & 2 & 176.785 & 0.00709924 \\
$S$ & 11 & 50 & 3 & 286.868 & 0.0980501 \\
$S$ & 11 & 50 & 4 & 122.471 & 0.0864266 \\
$S$ & 11 & 50 & 5 & 426.155 & 0.179799 \\
$S$ & 11 & 50 & 6 & 189.946 & 0.0648711 \\
$S$ & 11 & 50 & 7 & 125.197 & 0.107896 \\
$S$ & 11 & 50 & 8 & 88.2816 & 0.0973212 \\
$S$ & 11 & 50 & 9 & 124.645 & 0.201606 \\
$S$ & 11 & 50 & 10 & 69.7302 & 0.0264321 \\
$S$ & 11 & 50 & 11 & 117.93 & 0.168262 \\
$S$ & 11 & 50 & 12 & 87.3559 & 0.0498899 \\
$S$ & 11 & 50 & 13 & 87.7367 & 0.121808 \\
$S$ & 11 & 50 & 14 & 75.7696 & 0.0181803 \\
$S$ & 11 & 50 & 15 & 143.937 & 0.316835 \\
$S$ & 11 & 50 & 16 & 110.522 & 0.224954 \\
$S$ & 11 & 50 & 17 & 81.5673 & 0.094155 \\
$S$ & 11 & 50 & 18 & 124.095 & 0.273653 \\
$S$ & 11 & 50 & 19 & 158.675 & 0.141677 \\
$S$ & 11 & 50 & 20 & 124.234 & 0.183035
\end{tabular}

\begin{tabular}{|c|c|c|}
\hline $\mathrm{s}$ & $\mathrm{t}$ & a \\
\hline 240.404 & 1054.59 & 0.0000652872 \\
\hline 536.24 & 1579.72 & $-2.34917^{\prime} 10^{-6}$ \\
\hline 217.776 & 880.092 & -0.0000456116 \\
\hline 187.618 & 950.97 & -0.000017463 \\
\hline 48.0884 & 929.799 & -0.000020596 \\
\hline 200.311 & 613.744 & -0.000022652 \\
\hline 173.113 & 800. & $-1.94081 \cdot 10^{-6}$ \\
\hline 109.502 & 1681.63 & 0.000010329 \\
\hline 515.855 & 1169.45 & 0.0000170175 \\
\hline 312.45 & 729.85 & 1.4956187801802379 \\
\hline 380.341 & 1014.54 & -0.0000136878 \\
\hline 370.364 & 1228.3 & -0.0000170684 \\
\hline 537.395 & 1011.11 & $6.99256^{\prime} 10^{-6}$ \\
\hline 813.319 & 1386.4 & $-5.71543^{\prime} 10^{-6}$ \\
\hline 124.967 & 1000 & $-1.87846^{\prime} 10^{-6}$ \\
\hline 248.478 & 1093.34 & -0.000041715 \\
\hline 633.529 & 1093.85 & $4.71592^{\prime} 10^{-6}$ \\
\hline 191.016 & 1000.13 & $5.0456^{\prime} 10^{-6}$ \\
\hline 161.496 & 900 . & $-3.882^{\prime} 10^{-6}$ \\
\hline 244.497 & 1000 . & $-7.4225^{\prime} 10^{-7}$ \\
\hline
\end{tabular}


Sample 11 Scan 51:

\begin{tabular}{|c|c|c|c|c|c|c|c|c|c|}
\hline $\mathrm{s}$ & $\mathrm{T}$ & M & LL & w & $\mathrm{s}$ & $\mathrm{t}$ & a & $\circ$ erg & $f$ \\
\hline S 11 & 51 & 1 & 742.459 & 0.0263359 & 228.057 & 1024.41 & 0.0000641495 & 0.524058 & 0.0754753 \\
\hline S 11 & 51 & 2 & 178.175 & 0.00712479 & 495.734 & 1575.34 & $-1.70824^{\prime} 10^{-6}$ & 0.07755 & -0.453232 \\
\hline S 11 & 51 & 3 & 219.555 & 0.099866 & 234.977 & 887.744 & -0.0000323479 & 0.0781106 & -1.9327 \\
\hline S 11 & 51 & 4 & 434.968 & 0.181379 & 68.8467 & 889.902 & -0.0000293003 & 0.0845581 & 2.9013 \\
\hline S 11 & 51 & 5 & 312.265 & 0.0749341 & 365.652 & 828.375 & -0.0000341977 & 0.0629508 & -2.35933 \\
\hline S 11 & 51 & 6 & 62.7157 & 0.0259974 & 730.804 & 978.639 & $-1.6132035006666588^{\prime} 10^{-6}$ & 0.0104409 & 3.08562 \\
\hline S 11 & 51 & 7 & 160.172 & 0.113818 & 400.707 & 804.155 & -0.000106538 & 0.0238748 & 0.302941 \\
\hline S 11 & 51 & 8 & 138.516 & 0.102769 & 1066.62 & 1615.96 & 0.0000278802 & 0.0177777 & -0.428261 \\
\hline S 11 & 51 & 9 & 73.6078 & 0.194641 & 414.955 & 1213.36 & $-3.7811283425455805^{\prime} 10^{-6}$ & 0.00849143 & -1.05239 \\
\hline S 11 & 51 & 10 & 99.4582 & 0.0471954 & 496.047 & 1154.15 & -0.0000192105 & 0.0105244 & -1.15704 \\
\hline S 11 & 51 & 11 & 168.607 & 0.316884 & 259.339 & 1000 . & $-2.82781^{\prime} 10^{-7}$ & 0.0082875 & -0.314461 \\
\hline S 11 & 51 & 12 & 51.202 & 0.0162581 & 707.063 & 1249.71 & $-3.6868827225644264^{\prime} 10^{-6}$ & 0.00460974 & -2.25525 \\
\hline S 11 & 51 & 13 & 98.049 & 0.0835057 & 1317.19 & 1609.06 & $8.04764^{\prime} 10^{-6}$ & 0.0081949 & -0.653057 \\
\hline S 11 & 51 & 14 & 102.131 & 0.273686 & 185.665 & 1000 . & $5.0876721610238445^{\prime} 10^{-6}$ & 0.0070927 & -1.87427 \\
\hline S 11 & 51 & 15 & 139.507 & 0.245045 & 118.562 & 1200. & $1.1978812205938682^{\prime} 10^{-6}$ & 0.0092581 & 1.70937 \\
\hline S 11 & 51 & 16 & 68.5365 & 0.297036 & 182.484 & 691.995 & -0.0000353456 & 0.00409498 & -1.78579 \\
\hline S 11 & 51 & 17 & 99.2619 & 0.182003 & 926.054 & 1517.41 & -0.000021932 & 0.0055849 & 2.69952 \\
\hline S 11 & 51 & 18 & 40.8747 & 0.330305 & 461.527 & 950.897 & $5.37923^{\prime} 10^{-7}$ & 0.00214343 & -2.32888 \\
\hline S 11 & 51 & 19 & 72.0722 & 0.0582532 & 728.829 & 855.214 & $-8.49488^{\prime} 10^{-6}$ & 0.0035724 & 0.826137 \\
\hline 11 & 51 & 20 & 49.8251 & 0.211457 & 337.329 & 822.44 & $7.86766^{\prime} 10^{-6}$ & 0.00232339 & -0.711225 \\
\hline
\end{tabular}

\section{Sample 11 Scan 52:}

\begin{tabular}{|c|c|c|c|c|c|}
\hline 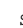 & & $\mathrm{T}$ & M & LL & w \\
\hline S & 11 & 52 & 1 & 749.831 & 0.0262505 \\
\hline & 11 & 52 & 2 & 178.896 & 0.00712586 \\
\hline & 11 & 52 & 3 & 229.633 & 0.0990752 \\
\hline & 11 & 52 & 4 & 380.983 & 0.179603 \\
\hline & 11 & 52 & 5 & 303.274 & 0.0705051 \\
\hline S & 11 & 52 & 6 & 94.8242 & 0.0889926 \\
\hline & 11 & 52 & 7 & 101.262 & 0.047438 \\
\hline S & 11 & 52 & 8 & 70.3719 & 0.107822 \\
\hline$S$ & 11 & 52 & 9 & 76.0371 & 0.0243214 \\
\hline$S$ & 11 & 52 & 10 & 76.7982 & 0.0585721 \\
\hline & 11 & 52 & 11 & 103.366 & 0.195319 \\
\hline S & 11 & 52 & 12 & 89.0602 & 0.0919456 \\
\hline & 11 & 52 & 13 & 173.46 & 0.316645 \\
\hline & 11 & 52 & 14 & 121.486 & 0.169824 \\
\hline & 11 & 52 & 15 & 135.011 & 0.141815 \\
\hline & 11 & 52 & 16 & 114.065 & 0.27367 \\
\hline & 11 & 52 & 17 & 147.479 & 0.242006 \\
\hline & 11 & 52 & 18 & 74.8674 & 0.0477696 \\
\hline 5 & 11 & 52 & 19 & 83.7076 & 0.220049 \\
\hline & 11 & 52 & 20 & 79.1784 & 0.117932 \\
\hline
\end{tabular}

\begin{tabular}{|c|c|c|c|c|}
\hline s & $\mathrm{t}$ & a & $\div$ erg & $f$ \\
\hline 230.141 & 1025.19 & 0.0000645305 & 0.527553 & 0.051488 \\
\hline 98.142 & 576.83 & $-1.8214949442558342^{\prime} 10^{-6}$ & 0.0772586 & -0.459423 \\
\hline 1.836 & 55.14 & -0.0000336708 & 0.0807983 & 1.31902 \\
\hline 3.9214 & 907.871 & -0.0000338639 & 0.0990986 & -0.36774 \\
\hline 184.059 & 800. & $-1.9377063231696275^{\prime} 10^{-6}$ & 0.0373648 & -0.709112 \\
\hline 58.122 & 1067.26 & $-8.26198^{\prime} 10^{-6}$ & 0.0159504 & 2.67146 \\
\hline 708.849 & 876.887 & 0.0000177203 & 0.0154988 & -2.04585 \\
\hline 5.135 & 1315.95 & $8.06898^{\prime} 10^{-7}$ & 0.00988277 & 1.42402 \\
\hline 8.779 & 916.657 & $7.7569^{\prime} 10^{-6}$ & 0.00992494 & -2.07632 \\
\hline 0.0486 & 1375.78 & 0.0000490502 & 0.00928683 & -0.16881 \\
\hline 410.246 & 1300.71 & $8.13343^{\prime} 10^{-6}$ & 0.0114251 & 0.332571 \\
\hline 80.835 & 1693.19 & -0.0000105959 & 0.00893984 & -1.55966 \\
\hline 122.008 & 999.989 & $3.5150352370483262^{\prime} 10^{-6}$ & 0.00942359 & -0.280618 \\
\hline 55.817 & 1044.29 & -0.0000139825 & 0.00990128 & -2.49227 \\
\hline 5.429 & 879.407 & -0.0000361166 & 0.00625217 & 2.86909 \\
\hline 177.526 & 1000 & $5.09035^{\prime} 10^{-6}$ & 0.00687111 & -1.89995 \\
\hline 212.441 & 1199.99 & $5.03451^{\prime} 10^{-6}$ & 0.00828441 & 1.80094 \\
\hline 273.628 & 517.011 & 0.0000200897 & 0.00398491 & -1.28171 \\
\hline 50.457 & 142.04 & $3.9570267130128065^{\prime} 10^{-6}$ & 0.00411607 & 1.9507 \\
\hline 54.484 & 2.63 & $5.43462^{\prime} 10^{-6}$ & 0.00358873 & 1.49616 \\
\hline
\end{tabular}

\section{Sample 11 Scan 53:}

\begin{tabular}{llllll}
$S$ & $T$ & $M$ & \multicolumn{1}{c}{ LL } & \multicolumn{1}{c}{ W } \\
$S$ & 11 & 53 & 1 & 751.63 & 0.0263529 \\
$S$ & 11 & 53 & 2 & 181.305 & 0.00713566 \\
$S$ & 11 & 53 & 3 & 444.819 & 0.087215 \\
$S$ & 11 & 53 & 4 & 181.012 & 0.0518945 \\
$S$ & 11 & 53 & 5 & 104.741 & 0.0867037 \\
$S$ & 11 & 53 & 6 & 126.533 & 0.108985 \\
$S$ & 11 & 53 & 7 & 159.888 & 0.179897 \\
$S$ & 11 & 53 & 8 & 39.9746 & 0.0168411 \\
$S$ & 11 & 53 & 9 & 154.533 & 0.1319 \\
$S$ & 11 & 53 & 10 & 59.7867 & 0.0283045 \\
$S$ & 11 & 53 & 11 & 98.8495 & 0.10121 \\
$S$ & 11 & 53 & 12 & 55.8284 & 0.0740243 \\
$S$ & 11 & 53 & 13 & 121.568 & 0.247027 \\
$S$ & 11 & 53 & 14 & 106.307 & 0.191826 \\
$S$ & 11 & 53 & 15 & 203.779 & 0.316642 \\
$S$ & 11 & 53 & 16 & 118.457 & 0.222634 \\
$S$ & 11 & 53 & 17 & 106.178 & 0.0573789 \\
$S$ & 11 & 53 & 18 & 246.801 & 0.169871 \\
$S$ & 11 & 53 & 19 & 124.881 & 0.203126 \\
$S$ & 11 & 53 & 20 & 153.522 & 0.283493
\end{tabular}

\begin{tabular}{|c|c|c|c|c|}
\hline s & $\mathrm{t}$ & a & $\frac{\circ}{\partial}$ erg & $f$ \\
\hline 228.04 & 1024.66 & 0.0000640079 & 0.528403 & 0.0801088 \\
\hline 493.915 & 1576.78 & $-1.73481 \cdot 10^{-6}$ & 0.0780673 & -0.45563 \\
\hline 131.785 & 824.698 & -0.000515821 & 0.14092 & -1.52321 \\
\hline 323.832 & 715.872 & $7.0324420559138465^{\prime} 10^{-6}$ & 0.0416358 & 0.702985 \\
\hline 713.116 & 1072.29 & $-1.13569^{\prime} 10^{-6}$ & 0.0208657 & 2.04218 \\
\hline 238.585 & 1100 . & $-7.28601^{\prime} 10^{-6}$ & 0.0223134 & 0.415626 \\
\hline 308.219 & 927.487 & -0.0000339324 & 0.024624 & 2.79213 \\
\hline 375.646 & 695.215 & 0.0000183531 & 0.00556537 & -1.57868 \\
\hline 154.656 & 900. & $-1.0801901820395352^{\prime} 10^{-7}$ & 0.0136624 & -0.802361 \\
\hline 396.315 & 1211.88 & -0.0000165296 & 0.00712527 & -2.51835 \\
\hline 240.834 & 1630.06 & $-7.21998^{\prime} 10^{-7}$ & 0.0108852 & -1.3315 \\
\hline 641.158 & 1050.21 & $-6.7704^{\prime} 10^{-6}$ & 0.00568863 & -0.854906 \\
\hline 213.048 & 1100 . & $-1.5394084021870257^{\prime} 10^{-7}$ & 0.0110252 & 1.40063 \\
\hline 130.642 & 1497.85 & $-3.42126^{\prime} 10^{-7}$ & 0.00501322 & -0.273333 \\
\hline 124.12 & 999.966 & $-2.97873^{\prime} 10^{-7}$ & 0.00859647 & -0.0439971 \\
\hline 381.383 & 966.577 & -0.0000136269 & 0.00831596 & -1.80104 \\
\hline 186.341 & 1338.49 & $2.3430481493254287^{\prime} 10^{-6}$ & 0.00666056 & 2.16242 \\
\hline 163.777 & 1100 & $-2.3957624411882476^{\prime} 10^{-} 6$ & 0.00768952 & 0.269261 \\
\hline .478 & 1000. & -0.0000216417 & 0.00511931 & -0.515678 \\
\hline 11 & 66.9 & 0.0000284885 & 0.0066401 & -2.46133 \\
\hline
\end{tabular}




\section{Sample 11 Scan 54:}

\begin{tabular}{|c|c|c|c|c|c|c|c|c|c|}
\hline $\mathrm{s}$ & $\mathrm{T}$ & M & LL & w & $\mathrm{s}$ & $\mathrm{t}$ & a & $\therefore$ erg & $\mathrm{f}$ \\
\hline s 11 & 54 & 1 & 861.851 & 0.0216783 & 240.53 & 1057.56 & 0.0000652671 & 0.577621 & -0.658007 \\
\hline S 11 & 54 & 2 & 211.794 & 0.00710888 & 535.407 & 1581.06 & $-2.3581299006704367^{\prime} 10^{-6}$ & 0.0805161 & -0.437725 \\
\hline S 11 & 54 & 3 & 395.011 & 0.0932511 & 69.0401 & 840.978 & 0.0000827303 & 0.111358 & 2.72327 \\
\hline S 11 & 54 & 4 & 80.1611 & 0.098061 & 252 . & 1000 . & $1.0592814440565459^{\prime} 10^{-10}$ & 0.0123116 & -2.29508 \\
\hline s 11 & 54 & 5 & 298.595 & 0.18729 & 259.716 & 1013.7 & -0.0000287326 & 0.0338384 & -0.617886 \\
\hline S 11 & 54 & 6 & 133.999 & 0.0574335 & 70.3434 & 570.364 & 0.000214024 & 0.0230425 & 1.59079 \\
\hline S 11 & 54 & 7 & 191.998 & 0.202293 & 235.378 & 1000 . & $1.9382135829540037^{\prime} 10^{-6}$ & 0.0171723 & -0.402777 \\
\hline S 11 & 54 & 8 & 83.0838 & 0.0888865 & 84.5822 & 1675.64 & -0.0000184667 & 0.0114376 & 0.2596 \\
\hline S 11 & 54 & 9 & 243.98 & 0.164086 & 165.755 & 1000 . & $-1.92476^{\prime} 10^{-6}$ & 0.0209751 & -2.24233 \\
\hline S 11 & 54 & 10 & 107.808 & 0.0456579 & 666.689 & 1043.2 & $-8.04447^{\prime} 10^{-6}$ & 0.0113496 & -2.56519 \\
\hline s 11 & 54 & 11 & 133.123 & 0.145067 & 250.017 & 1000 . & $-3.2403864683855144^{\prime} 10^{-7}$ & 0.0105295 & -1.13234 \\
\hline S 11 & 54 & 12 & 126.42 & 0.111728 & 486.63 & 1206.94 & $7.3704^{\prime} 10^{-6}$ & 0.0105898 & 0.937493 \\
\hline S 11 & 54 & 13 & 71.0969 & 0.0266163 & 915.849 & 1162.72 & $-9.88371 \cdot 10^{-7}$ & 0.00539307 & -2.15988 \\
\hline S 11 & 54 & 14 & 88.2555 & 0.0179897 & 481.189 & 1370.67 & $-2.2847365244367076^{\prime} 10^{-6}$ & 0.00618262 & 2.65295 \\
\hline s 11 & 54 & 15 & 73.3574 & 0.0895228 & 625.095 & 1082.17 & $8.46468^{\prime} 10^{-7}$ & 0.00473955 & 1.24991 \\
\hline S 11 & 54 & 16 & 86.3041 & 0.0671538 & 391.001 & 1383.61 & -0.00001991 & 0.00514863 & -0.395085 \\
\hline S 11 & 54 & 17 & 129.711 & 0.232868 & 237.352 & 1106.89 & -0.000031686 & 0.0068588 & -0.940222 \\
\hline s 11 & 54 & 18 & 230.304 & 0.316603 & 121.876 & 1000. & $-5.07877^{\prime} 10^{-6}$ & 0.00588746 & 0.0206965 \\
\hline S 11 & 54 & 19 & 7215 & .192768 & 6.484 & 1509.03 & .0000476122 & 0.00392788 & 2.44336 \\
\hline 11 & 54 & 20 & 180.146 & 0.278414 & 366.853 & 950.268 & 0.0000319978 & 0.00666748 & -0.717361 \\
\hline
\end{tabular}

\section{Sample 11 Scan 55:}

\begin{tabular}{llllll}
$S$ & $T$ & $M$ & \multicolumn{1}{c}{ LL } & \multicolumn{1}{c}{ W } \\
$S$ & 11 & 55 & 1 & 806.525 & 0.0261794 \\
$S$ & 11 & 55 & 2 & 200.753 & 0.00714224 \\
$S$ & 11 & 55 & 3 & 242.377 & 0.099246 \\
$S$ & 11 & 55 & 4 & 226.133 & 0.0699222 \\
$S$ & 11 & 55 & 5 & 75.4473 & 0.088666 \\
$S$ & 11 & 55 & 6 & 197.158 & 0.185166 \\
$S$ & 11 & 55 & 7 & 91.0021 & 0.0472727 \\
$S$ & 11 & 55 & 8 & 126.028 & 0.202128 \\
$S$ & 11 & 55 & 9 & 147.984 & 0.121017 \\
$S$ & 11 & 55 & 10 & 83.2749 & 0.0242893 \\
$S$ & 11 & 55 & 11 & 144.871 & 0.165311 \\
$S$ & 11 & 55 & 12 & 156.169 & 0.124898 \\
$S$ & 11 & 55 & 13 & 109.832 & 0.0579413 \\
$S$ & 11 & 55 & 14 & 89.734 & 0.216645 \\
$S$ & 11 & 55 & 15 & 73.5583 & 0.106791 \\
$S$ & 11 & 55 & 16 & 65.7208 & 0.105817 \\
$S$ & 11 & 55 & 17 & 74.2471 & 0.190426 \\
$S$ & 11 & 55 & 18 & 179.138 & 0.316458 \\
$S$ & 11 & 55 & 19 & 79.4673 & 0.0479988 \\
$S$ & 11 & 55 & 20 & 177.577 & 0.278138
\end{tabular}

$\begin{array}{lllll}\mathrm{S} 11 \quad 55 & 20 & 177.577 & 0.278138\end{array}$

\begin{tabular}{|c|c|c|c|c|}
\hline s & $\mathrm{t}$ & a & $\frac{\circ}{\partial}$ erg & $f$ \\
\hline 231.872 & 1027.14 & 0.0000647808 & 0.553593 & 0.027397 \\
\hline 496.085 & 1581.07 & $-1.8872266166888401 \cdot 10^{-6}$ & 0.0810281 & -0.483975 \\
\hline 229.232 & 861.322 & -0.0000348007 & 0.0782547 & 0.81214 \\
\hline 201.292 & 799.999 & 0 . & 0.035709 & -0.663505 \\
\hline 287.377 & 1059.31 & $-9.26436^{\prime} 10^{-6}$ & 0.0181846 & -2.73329 \\
\hline 267.395 & 998.927 & -0.0000391699 & 0.0413796 & 2.34122 \\
\hline 713.591 & 881.833 & 0.0000177231 & 0.016585 & -2.24349 \\
\hline 264.615 & 1030.52 & $-1.83845^{\prime} 10^{-6}$ & 0.0205277 & -0.198786 \\
\hline 767.132 & 1302.63 & 0.0000297802 & 0.0211219 & -3.05534 \\
\hline 486.974 & 917.289 & $7.9329^{\prime} 10^{-6}$ & 0.0105814 & -2.07847 \\
\hline 268.827 & 1063.13 & -0.0000197359 & 0.0164267 & 0.126308 \\
\hline 243.04 & 825.441 & -0.0000657563 & 0.0152424 & -1.95263 \\
\hline 96.3146 & 1379.82 & 0.0000418564 & 0.00938001 & -0.332227 \\
\hline 483.949 & 860.231 & -0.0000314058 & 0.00693464 & -2.22378 \\
\hline 913.837 & 1445.65 & 0.000051824 & 0.00523833 & 2.35057 \\
\hline 1277.62 & 1748.52 & $8.06462^{\prime} 10^{-7}$ & 0.00436515 & -0.404055 \\
\hline 803.628 & 1322.71 & $3.4547123823931664^{\prime} 10^{-6}$ & 0.00459836 & 1.846 \\
\hline 120.47 & 1000 & $-1.9387073092477668^{\prime} 10^{-6}$ & 0.00718578 & -0.0404861 \\
\hline 284.499 & 508.546 & 0.0000213519 & 0.00400878 & -0.858933 \\
\hline 46.175 & 950.462 & 0.0000335842 & 0.00788587 & -0.854426 \\
\hline
\end{tabular}

$0.00788587-0.854426$ 


\section{Sample 15 Scan 1:}

\begin{tabular}{|c|c|c|c|c|c|c|c|c|c|c|}
\hline s & 5 & $\mathrm{~T}$ & M & LL & w & $\mathrm{s}$ & $\mathrm{t}$ & a & $\frac{\circ}{\mathrm{erg}}$ & $f$ \\
\hline 5 & 15 & 1 & 1 & 1798.52 & 0.0235159 & 238.082 & 1109.88 & 0.0000572022 & 0.834456 & -0.152212 \\
\hline & 15 & 1 & 2 & 530.738 & 0.0061055 & 530.243 & 1529.1 & $-4.770311^{\prime} 10^{-6}$ & 0.0677865 & 0.650407 \\
\hline & 15 & 1 & 3 & 904.121 & 0.0870276 & 240.281 & 954.07 & -0.0000162572 & 0.0301109 & -2.92659 \\
\hline & 15 & 1 & 4 & 221.09 & 0.0236119 & 803.575 & 964.914 & $-4.44141 \cdot 10^{-6}$ & 0.0131802 & -1.50022 \\
\hline & 15 & 1 & 5 & 191.901 & 0.0717779 & 616.653 & 1083.02 & $-1.2125313617793825^{\prime} 10^{-6}$ & 0.00930017 & 2.9218 \\
\hline & 15 & 1 & 6 & 143.98 & 0.0591956 & 738.699 & 958.397 & $-3.4431637017362693^{\prime} 10^{-6}$ & 0.00589507 & -0.925602 \\
\hline & 15 & 1 & 7 & 141.322 & 0.0398842 & 578.674 & 954.807 & $7.84228^{\prime} 10^{-6}$ & 0.00501685 & -2.20464 \\
\hline & 15 & 1 & 8 & 406.625 & 0.13619 & 156.707 & 900.004 & $-1.0000507344281825^{\prime} 10^{-6}$ & 0.00755405 & 0.17532 \\
\hline & 15 & 1 & 9 & 130.333 & 0.0290658 & 297.8 & 550.654 & $-5.31929^{\prime} 10^{-7}$ & 0.00311554 & 1.5298 \\
\hline & 15 & 1 & 10 & 108.834 & 0.0178834 & 341.585 & 1369.03 & $-2.0124729831846956^{\prime} 10^{-7}$ & 0.00230789 & -1.83951 \\
\hline & 15 & 1 & 11 & 122.747 & 0.098998 & 801.265 & 1026.94 & $-3.24482^{\prime} 10^{-6}$ & 0.00231864 & 2.17684 \\
\hline & 15 & 1 & 12 & 285.377 & 0.118412 & 176.365 & 899.931 & $-9.96492^{\prime} 10^{-7}$ & 0.00440251 & -1.53629 \\
\hline
\end{tabular}

\section{Sample 15 Scan 2:}

\begin{tabular}{llllll}
$S$ & $T$ & $M$ & \multicolumn{1}{c}{ LL } & \multicolumn{1}{c}{ W } \\
$\mathrm{S}$ & 15 & 2 & 1 & 1749.34 & 0.0230709 \\
$\mathrm{~S}$ & 15 & 2 & 2 & 527.75 & 0.00620521 \\
$\mathrm{~S}$ & 15 & 2 & 3 & 413.947 & 0.0862769 \\
$\mathrm{~S}$ & 15 & 2 & 4 & 231.855 & 0.023794 \\
$\mathrm{~S}$ & 15 & 2 & 5 & 126.708 & 0.059504 \\
$\mathrm{~S}$ & 15 & 2 & 6 & 237.764 & 0.073603 \\
$\mathrm{~S}$ & 15 & 2 & 7 & 146.405 & 0.0393275 \\
$\mathrm{~S}$ & 15 & 2 & 8 & 410.427 & 0.136317 \\
$\mathrm{~S}$ & 15 & 2 & 9 & 94.9515 & 0.0179312 \\
$\mathrm{~S}$ & 15 & 2 & 10 & 126.292 & 0.0352605 \\
$\mathrm{~S}$ & 15 & 2 & 11 & 435.407 & 0.11559 \\
$\mathrm{~S}$ & 15 & 2 & 12 & 145.891 & 0.096936
\end{tabular}

\begin{tabular}{|c|c|c|c|c|}
\hline s & $t$ & a & $\frac{\circ}{6}$ erg & $f$ \\
\hline 9.711 & 1111.59 & 0.0000581722 & 0.826112 & -0.249982 \\
\hline 36.865 & 1527.68 & $-5.19064^{\prime} 10^{-6}$ & 0.0709566 & 0.641525 \\
\hline 99.598 & 908.577 & -0.0000254719 & 0.0345217 & 1.13817 \\
\hline 24.647 & 969.238 & $-4.31808^{\prime} 10^{-6}$ & 0.0139318 & -1.66912 \\
\hline 21.854 & 987.855 & $-3.15421 \cdot 10^{-6}$ & 0.0063534 & -2.74384 \\
\hline 54.577 & 1200. & $-1 .^{\prime} 10^{-6}$ & 0.00740538 & 0.695322 \\
\hline 33.675 & 666.627 & -0.0000481224 & 0.00539691 & -2.15771 \\
\hline 31.475 & 899.989 & $-1 \cdot 10^{-6}$ & 0.00760185 & 0.182215 \\
\hline 21.846 & 1376.57 & $-1.6568615269637439^{\prime} 10^{-6}$ & 0.00241198 & -1.92071 \\
\hline 92.855 & 1163.78 & $-2.3505476408644155^{\prime} 10^{-6}$ & 0.00287296 & 2.59502 \\
\hline 3.834 & 1018.52 & $7.88858^{\prime} 10^{-6}$ & 0.00447359 & -3.07149 \\
\hline 5.716 & 640.367 & $-2.70607^{\prime} 10^{-7}$ & 0.00228948 & 2.09394 \\
\hline
\end{tabular}

\section{Sample 15 Scan 3:}

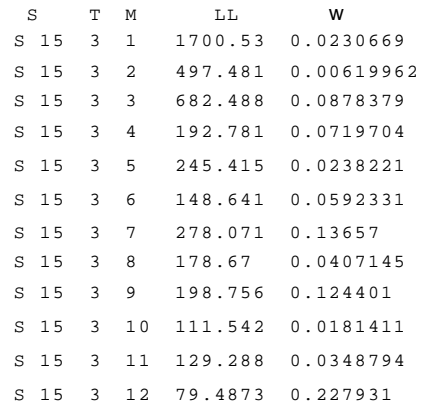

Sample 15 Scan 4:

\begin{tabular}{|c|c|c|c|c|}
\hline $\mathrm{s}$ & $t$ & a & $\frac{\circ}{\partial}$ erg & $f$ \\
\hline 239.927 & 1110.53 & 0.0000582876 & 0.817413 & -0.252548 \\
\hline 35.828 & 1525.8 & $-5.19019^{\prime} 10^{-6}$ & 0.0712382 & 0.643393 \\
\hline 85.839 & 961.554 & -0.0000159593 & 0.0321423 & 2.57055 \\
\hline 586.346 & 1025.37 & $-7.84263^{\prime} 10^{-7}$ & 0.013701 & 0.652405 \\
\hline 821.3 & 969.503 & $-4.2991^{\prime} 10^{-6}$ & 0.0140233 & -1.70154 \\
\hline 755.282 & 966.285 & $-3.3608035519223875^{\prime} 10^{-6}$ & 0.00696284 & -1.5373 \\
\hline 156.71 & 900. & $-1.0003193236954523^{\prime} 10^{-6}$ & 0.00928255 & 0.167167 \\
\hline 345.93 & 677.911 & -0.0000259623 & 0.00559007 & -2.8391 \\
\hline 247.244 & 1007.17 & $-8.0749^{\prime} 10^{-6}$ & 0.00434486 & -2.0191 \\
\hline 910.21 & 1313.77 & $-1.1722147971495815^{\prime} 10^{-6}$ & 0.00255744 & -0.776037 \\
\hline 246.024 & 1162.04 & $-7.77776^{\prime} 10^{-6}$ & 0.00262812 & 2.71414 \\
\hline 53.65 & 193.19 & $8.84097^{\prime} 10^{-6}$ & 0.00145501 & 3.02786 \\
\hline
\end{tabular}

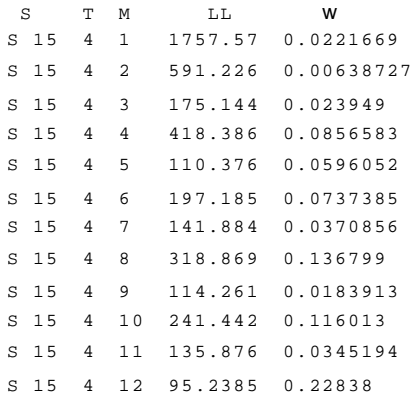

\begin{tabular}{|c|c|c|c|c|}
\hline s & $\mathrm{t}$ & a & $\frac{\circ}{\partial}$ erg & $f$ \\
\hline 42.072 & 1119.11 & 0.0000581589 & 0.827536 & -0.401153 \\
\hline 545.711 & 1540.56 & $-5.38122^{\prime} 10^{-6}$ & 0.0765875 & 0.574241 \\
\hline 21.817 & 980.395 & $-4.2624436758733335^{\prime} 10^{-6}$ & 0.0152276 & -1.95944 \\
\hline 314.841 & 891.344 & -0.000026359 & 0.0271961 & 2.5805 \\
\hline 722.594 & 1002.25 & $-3.3528^{\prime} 10^{-6}$ & 0.00547068 & 2.75221 \\
\hline 174.008 & 1200. & $-1.0139150640813647^{\prime} 10^{-6}$ & 0.00598628 & 0.689327 \\
\hline 306.444 & 610.127 & -0.000024775 & 0.00540925 & -0.210961 \\
\hline 35.516 & 899.998 & $-1 \cdot 10^{-6}$ & 0.00804015 & 0.0173026 \\
\hline 002.2 & 1385. & $-1.24372^{\prime} 10^{-6}$ & 0.00296321 & -2.00687 \\
\hline 56.158 & 865.322 & -0.0000282145 & 0.00525021 & 2.64682 \\
\hline 280.706 & 1169.6 & $-7.15664^{\prime} 10^{-6}$ & 0.0024426 & 2.52613 \\
\hline 14.959 & 1167.51 & $9.46802^{\prime} 10^{-6}$ & 0.00152464 & 2.48629 \\
\hline
\end{tabular}




\section{Sample 15 Scan 5:}

\begin{tabular}{|c|c|c|c|c|c|c|c|c|c|c|}
\hline $\mathrm{s}$ & 5 & $\mathrm{~T}$ & M & LL & w & $\mathrm{s}$ & $\mathrm{t}$ & a & $\%$ erg & $\mathrm{f}$ \\
\hline $\mathrm{s}$ & 15 & 5 & 1 & 1766.11 & 0.0220984 & 242.76 & 1120.33 & 0.0000578526 & 0.829002 & -0.4065 \\
\hline $\mathrm{s}$ & 15 & 5 & 2 & 606.026 & 0.00628586 & 539.629 & 1538.82 & $-5.06118^{\prime} 10^{-6}$ & 0.0772926 & 0.582966 \\
\hline$S$ & 15 & 5 & 3 & 179.437 & 0.0239908 & 837.614 & 983.137 & $-4.20957^{\prime} 10^{-6}$ & 0.0151962 & -2.01075 \\
\hline S & 15 & 5 & 4 & 417.037 & 0.0855775 & 315.505 & 890.735 & -0.0000263667 & 0.0263633 & 2.66798 \\
\hline $\mathrm{s}$ & 15 & 5 & 5 & 116.568 & 0.0596112 & 711.938 & 1003.02 & $-3.25515^{\prime} 10^{-6}$ & 0.00560577 & 2.72256 \\
\hline $\mathrm{s}$ & 15 & 5 & 6 & 223.227 & 0.0735968 & 196.416 & 1200.03 & $-1.0148066470096308^{\prime} 10^{-6}$ & 0.00583878 & 0.728233 \\
\hline $\mathrm{s}$ & 15 & 5 & 7 & 150.774 & 0.0370649 & 308.129 & 609.546 & -0.0000250522 & 0.00552876 & -0.161007 \\
\hline $\mathrm{s}$ & 15 & 5 & 8 & 294.773 & 0.136792 & 105.72 & 900. & $-1.0000000071900872^{\prime} 10^{-6}$ & 0.00736092 & 0.0130619 \\
\hline $\mathrm{s}$ & 15 & 5 & 9 & 123.2 & 0.0180753 & 958.093 & 1359.39 & $-9.24617^{\prime} 10^{-7}$ & 0.00308484 & -1.51357 \\
\hline S & 15 & 5 & 10 & 113.146 & 0.0342857 & 288.83 & 1171.62 & $-7.16626^{\prime} 10^{-6}$ & 0.00251676 & 2.46296 \\
\hline 3 & 15 & 5 & 11 & 236.633 & 0.124054 & 206.218 & 1047.97 & $-8.99068^{\prime} 10^{-6}$ & 0.0034781 & -0.84037 \\
\hline S & 15 & 5 & 12 & 130.237 & 0.0964105 & 62.743 & 40.534 & $4.88311^{\prime} 10^{-6}$ & 0.00185651 & 1.97282 \\
\hline
\end{tabular}

\section{Sample 15 Scan 6:}

\begin{tabular}{|c|c|c|c|c|c|c|c|c|c|c|}
\hline s. & & $\mathrm{T}$ & M & LL & w & $\mathrm{s}$ & $\mathrm{t}$ & a & 음 erg & $f$ \\
\hline & 15 & 6 & 1 & 1861.59 & 0.0216169 & 242.902 & 1127.91 & 0.0000572091 & 0.844575 & -0.472875 \\
\hline $\mathrm{s}$ & 15 & 6 & 2 & 736.423 & 0.00624808 & 543.745 & 1544.99 & $-4.99205^{\prime} 10^{-6}$ & 0.0804744 & 0.582969 \\
\hline$S$ & 15 & 6 & 3 & 241.146 & 0.0239747 & 831.665 & 986.938 & $-4.18687^{\prime} 10^{-6}$ & 0.0157741 & -2.03397 \\
\hline $\mathrm{S}$ & 15 & 6 & 4 & 385.884 & 0.0848564 & 327.959 & 876.826 & -0.0000270407 & 0.0185163 & -2.24367 \\
\hline$S$ & 15 & 6 & 5 & 120.539 & 0.059744 & 701.668 & 1012.75 & $-3.24108^{\prime} 10^{-6}$ & 0.00446507 & 2.3732 \\
\hline$S$ & 15 & 6 & 6 & 165.365 & 0.0408401 & 315.057 & 692.308 & -0.0000589202 & 0.00531242 & -2.92679 \\
\hline $\mathrm{s}$ & 15 & 6 & 7 & 111.993 & 0.0176913 & 425.114 & 1430.09 & $-1.5740239002193555^{\prime} 10^{-6}$ & 0.00312313 & -2.66384 \\
\hline S & 15 & 6 & 8 & 181.242 & 0.0714954 & 71.6286 & 1198.99 & -0.000192777 & 0.00436867 & 1.40451 \\
\hline$S$ & 15 & 6 & 9 & 386.306 & 0.130117 & 234.812 & 1000.12 & $-1 \cdot 10^{-6}$ & 0.00481428 & -0.708115 \\
\hline $\mathrm{s}$ & 15 & 6 & 10 & 152.192 & 0.0500568 & 494.47 & 1196.57 & 0.000175443 & 0.00242418 & 1.47191 \\
\hline$S$ & 15 & 6 & 11 & 240.583 & 0.0478712 & 2259.89 & 961.789 & -0.0000179086 & 0.00315253 & -2.01881 \\
\hline & 15 & 6 & 12 & 80.9307 & 0.00949718 & 557.061 & 327.48 & -0.0000352953 & 0.000900783 & 2.1978 \\
\hline
\end{tabular}

\section{Sample 15 Scan 7:}

\begin{tabular}{|c|c|c|c|c|c|c|c|c|c|c|}
\hline & & $\mathrm{T}$ & M & LL & w & s & $\mathrm{t}$ & a & $\frac{\circ}{\circ}$ erg & $f$ \\
\hline & 15 & 7 & 1 & 1772.23 & 0.0225201 & 243.019 & 1117.71 & 0.0000570743 & 0.830046 & -0.31740 \\
\hline & 15 & 7 & 2 & 596.505 & 0.00625899 & 538.955 & 1543.21 & $-4.93529^{\prime} 10^{-6}$ & 0.0758985 & 0.578084 \\
\hline & 15 & 7 & 3 & 152.468 & 0.0283091 & 1541.16 & 1441.38 & $-4.79339^{\prime} 10^{-6}$ & 0.0131157 & -1.28033 \\
\hline & 15 & 7 & 4 & 405.453 & 0.0847998 & 261.53 & 869.478 & -0.0000284847 & 0.0265398 & -1.7026 \\
\hline & 15 & 7 & 5 & 137.711 & 0.0593579 & 709.83 & 961.155 & $-3.26875^{\prime} 10^{-6}$ & 0.00682896 & -1.04894 \\
\hline & 15 & 7 & 6 & 160.29 & 0.0407355 & 521.215 & 698.412 & -0.0000553695 & 0.00685019 & 2.98521 \\
\hline & 15 & 7 & 7 & 142.084 & 0.0700687 & 437.98 & 1250.7 & $-4.83151 \cdot 10^{-6}$ & 0.00519409 & -2.89959 \\
\hline & 15 & 7 & 8 & 96.7101 & 0.0181736 & 1059.82 & 1490.14 & $-1.8863068258770428^{\prime} 10^{-6}$ & 0.00313608 & 2.41678 \\
\hline & 15 & 7 & 9 & 114.995 & 0.0318305 & 975.794 & 995.118 & $-5.44993^{\prime} 10^{-6}$ & 0.00335464 & 2.03609 \\
\hline & 15 & 7 & 10 & 383.182 & 0.133023 & 243.255 & 1000 & $-9.99989^{\prime} 10^{-7}$ & 0.00656323 & -0.708674 \\
\hline & 15 & 7 & 11 & 121.327 & 0.0941171 & 124.971 & 668.997 & $2.6482688693529817^{\prime} 10^{-6}$ & 0.00204949 & -0.589774 \\
\hline & 15 & 7 & 12 & 162.195 & 0.116943 & 213.426 & 1000 . & $-1.0000009788210643^{\prime} 10^{-6}$ & 0.00233647 & -0.594543 \\
\hline
\end{tabular}

\section{Sample 15 Scan 8:}

\begin{tabular}{llllll} 
S & T & $M$ & LL & \multicolumn{1}{c}{$W$} \\
$S$ & 15 & 8 & 1 & 1616.45 & 0.0233463 \\
$S$ & 15 & 8 & 2 & 440.621 & 0.00624281 \\
$S$ & 15 & 8 & 3 & 422.533 & 0.0876575 \\
$S$ & 15 & 8 & 4 & 140.937 & 0.0719001 \\
$S$ & 15 & 8 & 5 & 185.317 & 0.0237038 \\
$S$ & 15 & 8 & 6 & 139.601 & 0.0590921 \\
$S$ & 15 & 8 & 7 & 530.916 & 0.132375 \\
$S$ & 15 & 8 & 8 & 151.201 & 0.0406881 \\
$S$ & 15 & 8 & 9 & 123.219 & 0.102007 \\
$S$ & 15 & 8 & 10 & 183.315 & 0.0241852 \\
$S$ & 15 & 8 & 11 & 152.12 & 0.229569 \\
$S$ & 15 & 8 & 12 & 240.001 & 0.150288
\end{tabular}

\begin{tabular}{|c|c|c|c|c|}
\hline $\mathrm{s}$ & $\mathrm{t}$ & a & $\frac{\circ}{b} \operatorname{erg}$ & $f$ \\
\hline 22.585 & 1107.8 & 0.0000568971 & 0.801397 & -0.169829 \\
\hline 35.051 & 1540.53 & $-4.87808^{\prime} 10^{-6}$ & 0.0704165 & 0.579929 \\
\hline 23.252 & 962.008 & -0.0000178359 & 0.0369095 & 2.46007 \\
\hline 53.701 & 1089.72 & $-8.44046^{\prime} 10^{-7}$ & 0.0118291 & 2.22725 \\
\hline 29.229 & 967.36 & $-4.39622^{\prime} 10^{-6}$ & 0.0132208 & -1.63844 \\
\hline 18.81 & 956.609 & $-3.05884546842334427^{\prime} 10^{-6}$ & 0.00846002 & -1.0618 \\
\hline 39.224 & 1000 . & $-1.0000000095038466^{\prime} 10^{-6}$ & 0.0174284 & -0.910285 \\
\hline 42.908 & 672.629 & -0.0000263307 & 0.00547849 & -2.71784 \\
\hline 07.865 & 1317.84 & 0.0000149887 & 0.00389096 & -3.11375 \\
\hline 60.749 & 1217.17 & 0.0000562025 & 0.00496987 & 0.521388 \\
\hline 9.867 & 1000 . & $-9.99968^{\prime} 10^{-7}$ & 0.00187655 & -2.77274 \\
\hline 077 & 1050.57 & 0000649372 & .00443059 & .8900 \\
\hline
\end{tabular}




\section{Sample 15 Scan 9:}

\begin{tabular}{|c|c|c|c|c|c|c|c|c|c|c|}
\hline $\mathrm{s}$ & 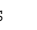 & $\mathrm{T}$ & M & LL & w & $\mathrm{s}$ & $\mathrm{t}$ & a & 응 erg & $f$ \\
\hline s & 15 & 9 & 1 & 1659.12 & 0.023192 & 242.681 & 1109.94 & 0.0000566479 & 0.809693 & -0.193795 \\
\hline S & 15 & 9 & 2 & 481.367 & 0.00626366 & 536.511 & 1545.29 & $-4.86722^{\prime} 10^{-6}$ & 0.0722977 & 0.564583 \\
\hline$S$ & 15 & 9 & 3 & 346.216 & 0.0872685 & 247.38 & 954.248 & -0.00001823 & 0.0334031 & -3.0949 \\
\hline S & 15 & 9 & 4 & 176.575 & 0.0237579 & 837.152 & 974.273 & $-4.4265605832329755^{\prime} 10^{-6}$ & 0.0134701 & -1.7808 \\
\hline S & 15 & 9 & 5 & 151.779 & 0.071866 & 664.649 & 1123.21 & $-9.93652^{\prime} 10^{-7}$ & 0.0098214 & -0.117898 \\
\hline $\mathrm{S}$ & 15 & 9 & 6 & 146.228 & 0.0590622 & 727.69 & 953.09 & $-3.0146434075988425^{\prime} 10^{-6}$ & 0.00815173 & -0.798196 \\
\hline s & 15 & 9 & 7 & 111.291 & 0.0406202 & 354.267 & 671.193 & -0.0000264277 & 0.00545248 & -2.6429 \\
\hline S & 15 & 9 & 8 & 244.387 & 0.13009 & 257.486 & 1000 & $-9.84413^{\prime} 10^{-7}$ & 0.00969229 & -0.781929 \\
\hline $\mathrm{s}$ & 15 & 9 & 9 & 114.909 & 0.0991983 & 775.776 & 1033.37 & $-2.42142^{\prime} 10^{-6}$ & 0.00397577 & 1.17388 \\
\hline $\mathrm{s}$ & 15 & 9 & 10 & 172.329 & 0.0112575 & 377.533 & 1335.12 & 0.000055962 & 0.00516834 & -1.51445 \\
\hline S & 15 & 9 & 11 & 300.68 & 0.146221 & 173.041 & 900. & $-9.37278^{\prime} 10^{-7}$ & 0.0056048 & 0.485008 \\
\hline s & 15 & 9 & 12 & 210.285 & 0.117247 & 341.95 & 900.225 & 0.0000170163 & 0.00414188 & -2.01323 \\
\hline
\end{tabular}

\section{Sample 15 Scan 10:}

\begin{tabular}{|c|c|c|c|c|c|c|c|c|c|c|}
\hline $\mathrm{s}$ & 5 & $\mathrm{~T}$ & M & LL & w & $\mathrm{s}$ & $\mathrm{t}$ & a & $\div$ erg & $f$ \\
\hline s & 15 & 10 & 1 & 1697.65 & 0.023142 & 243.198 & 1111.68 & 0.0000561775 & 0.816887 & -0.192249 \\
\hline $\mathrm{s}$ & 15 & 10 & 2 & 514.842 & 0.00624718 & 537.289 & 1548.69 & $-4.78122^{\prime} 10^{-6}$ & 0.0732168 & 0.561628 \\
\hline S & 15 & 10 & 3 & 359.652 & 0.0866243 & 284.307 & 906. & -0.0000274143 & 0.0327456 & 1.32869 \\
\hline $\mathrm{S}$ & 15 & 10 & 4 & 198.782 & 0.0236943 & 841.748 & 971.408 & $-4.4476622943162605^{\prime} 10^{-6}$ & 0.0136383 & -1.68121 \\
\hline $\mathrm{S}$ & 15 & 10 & 5 & 135.755 & 0.0592373 & 687.051 & 957.058 & $-3.0213460778474225^{\prime} 10^{-6}$ & 0.00787182 & -0.92734 \\
\hline$S$ & 15 & 10 & 6 & 127.993 & 0.074217 & 282.282 & 1210.07 & $6.92695^{\prime} 10^{-6}$ & 0.00650423 & -0.176254 \\
\hline s & 15 & 10 & 7 & 126.309 & 0.0359006 & 330.822 & 590.583 & -0.000024858 & 0.00565214 & 0.418681 \\
\hline $\mathrm{s}$ & 15 & 10 & 8 & 545.849 & 0.127733 & 145.038 & 1000 . & $-1 .^{\prime} 10^{-6}$ & 0.012397 & -0.811367 \\
\hline $\mathrm{S}$ & 15 & 10 & 9 & 154.776 & 0.0294584 & 88.7028 & 1212.36 & 0.0000409105 & 0.00424227 & 0.820247 \\
\hline $\mathrm{S}$ & 15 & 10 & 10 & 97.0381 & 0.0966737 & 208.261 & 640.595 & $4.5180819268990305^{\prime} 10^{-6}$ & 0.00234078 & 1.98208 \\
\hline$s$ & 15 & 10 & 11 & 81.3582 & 0.0154327 & 89.3299 & 1560.42 & 0.000078521 & 0.00179486 & 1.26199 \\
\hline & & & & 05.673 & 150607 & 57.636 & 00 & -6 & 0.00328969 & -2.0291 \\
\hline
\end{tabular}

\section{Sample 15 Scan 11:}

\begin{tabular}{|c|c|c|c|c|c|c|c|c|c|c|}
\hline s. & & $\mathrm{T}$ & M & LL & w & $\mathrm{s}$ & $\mathrm{t}$ & a & $\div$ erg & $f$ \\
\hline $\mathrm{s}$ & 15 & 11 & 1 & 1587.39 & 0.023514 & 242.556 & 1105.9 & 0.0000562456 & 0.795542 & -0.127489 \\
\hline $\mathrm{s}$ & 15 & 11 & 2 & 419.977 & 0.00622246 & 536.702 & 1544.5 & $-4.80692^{\prime} 10^{-6}$ & 0.0697357 & 0.574335 \\
\hline $\mathrm{s}$ & 15 & 11 & 3 & 325.882 & 0.0878503 & 257.32 & 962.696 & -0.0000189255 & 0.0366102 & 2.31952 \\
\hline $\mathrm{s}$ & 15 & 11 & 4 & 110.872 & 0.0718382 & 683.394 & 1135.43 & $-1.0260671938065485^{\prime} 10^{-6}$ & 0.0101468 & -1.11059 \\
\hline $\mathrm{S}$ & 15 & 11 & 5 & 161.888 & 0.02361 & 831.308 & 961.095 & $-4.41335^{\prime} 10^{-6}$ & 0.0129344 & -1.50176 \\
\hline$S$ & 15 & 11 & 6 & 125.945 & 0.0590099 & 725.038 & 948.681 & $-2.9422476099630805^{\prime} 10^{-6}$ & 0.00870943 & -0.638119 \\
\hline $\mathrm{s}$ & 15 & 11 & 7 & 72.9105 & 0.0993524 & 753.37 & 1040.15 & $-1.8329653905201133^{\prime} 10^{-6}$ & 0.00456277 & 0.222897 \\
\hline$S$ & 15 & 11 & 8 & 374.209 & 0.137636 & 136.353 & 900.026 & $-9.95853^{\prime} 10^{-7}$ & 0.0140487 & -0.107836 \\
\hline$S$ & 15 & 11 & 9 & 260.127 & 0.120936 & 232.862 & 996.221 & -0.0000188108 & 0.00837976 & -0.593252 \\
\hline$S$ & 15 & 11 & 10 & 163.743 & 0.0363052 & 342.819 & 593.622 & -0.000025856 & 0.00572416 & 0.241738 \\
\hline$S$ & 15 & 11 & 11 & 44.095 & 0.0281828 & 145.443 & 1206.59 & 0.0000545846 & 0.00431756 & 0.799979 \\
\hline$s$ & 15 & 11 & 12 & 105.36 & 0.231355 & 461.011 & 1146.3 & -0.0000105921 & 0.00278439 & 1.28529 \\
\hline
\end{tabular}

\section{Sample 15 Scan 12:}

\begin{tabular}{llllll}
\multicolumn{2}{c}{$S$} & $T$ & $M$ & \multicolumn{1}{c}{$L \mathrm{~L}$} & \multicolumn{1}{c}{$W$} \\
$\mathrm{~S}$ & 15 & 12 & 1 & 1605.21 & 0.0235172 \\
$\mathrm{~S}$ & 15 & 12 & 2 & 435.754 & 0.00623464 \\
$\mathrm{~S}$ & 15 & 12 & 3 & 723.321 & 0.0877102 \\
$\mathrm{~S}$ & 15 & 12 & 4 & 117.267 & 0.0718778 \\
$\mathrm{~S}$ & 15 & 12 & 5 & 170.315 & 0.0235511 \\
$\mathrm{~S}$ & 15 & 12 & 6 & 129.661 & 0.0589548 \\
$\mathrm{~S}$ & 15 & 12 & 7 & 294.688 & 0.138041 \\
$\mathrm{~S}$ & 15 & 12 & 8 & 164.67 & 0.12331 \\
$\mathrm{~S}$ & 15 & 12 & 9 & 150.209 & 0.0362911 \\
$\mathrm{~S}$ & 15 & 12 & 10 & 114.576 & 0.0991806 \\
$\mathrm{~S}$ & 15 & 12 & 11 & 152.509 & 0.0288434 \\
$\mathrm{~S}$ & 15 & 12 & 12 & 74.3171 & 0.112371
\end{tabular}

\begin{tabular}{|c|c|c|}
\hline $\mathrm{s}$ & $\mathrm{t}$ & a \\
\hline 41.899 & 1106.54 & 0.0000561649 \\
\hline 38.057 & 1547.1 & $-4.80197^{\prime} 10^{-6}$ \\
\hline 1.043 & 957.573 & -0.0000188124 \\
\hline 3.693 & 1128.08 & $-9.1891^{\prime} 10^{-7}$ \\
\hline 2.661 & 956.323 & $-4.37182 \cdot 10^{-6}$ \\
\hline 5.635 & 946.053 & $-2.9605330269058734^{\prime} 10^{-6}$ \\
\hline .659 & 899.991 & $-1.0145592421540297^{\prime} 10^{-6}$ \\
\hline 3.29 & 952.302 & $-8.54794^{\prime} 10^{-6}$ \\
\hline 8.032 & 592.684 & -0.0000260702 \\
\hline 3.294 & 1016.86 & $-2.16331 \cdot 10^{-6}$ \\
\hline .399 & 1206. & 0.0000539239 \\
\hline .364 & 971.875 & $-7.36609^{\prime} 10^{-6}$ \\
\hline
\end{tabular}

\begin{tabular}{ll}
\multicolumn{1}{c}{$\frac{\mathrm{o}}{\mathrm{O}} \mathrm{erg}$} & \multicolumn{1}{c}{$f$} \\
0.799153 & -0.127435 \\
0.0705428 & 0.56584 \\
0.0354587 & 2.81678 \\
0.0103334 & -0.536816 \\
0.0130061 & -1.3841 \\
0.00851752 & -0.436255 \\
0.0133416 & -0.0745742 \\
0.0070835 & -1.75419 \\
0.00573208 & 0.299835 \\
0.00382862 & 2.68663 \\
0.00446098 & 0.836793 \\
0.00193812 & 2.61329
\end{tabular}




\section{Sample 15 Scan 13:}

\begin{tabular}{|c|c|c|c|c|c|c|c|c|c|}
\hline $\mathrm{s}$ & $\mathrm{T}$ & M & LL & w & $\mathrm{s}$ & $t$ & a & $\therefore$ erg & $f$ \\
\hline S 15 & 13 & 1 & 1595.55 & 0.0235466 & 242.051 & 1106.38 & 0.0000559883 & 0.797203 & -0.117546 \\
\hline S 15 & 13 & 2 & 424.641 & 0.00620536 & 537.014 & 1545.63 & $-4.779311^{\prime} 10^{-6}$ & 0.0697677 & 0.579111 \\
\hline 15 & 13 & 3 & 310.605 & 0.0861083 & 261.432 & 925.476 & -0.0000228492 & 0.0351184 & -0.604832 \\
\hline S 15 & 13 & 4 & 145.083 & 0.0235454 & 826.821 & 956.894 & $-4.40999^{\prime} 10^{-6}$ & 0.0130228 & -1.39156 \\
\hline S 15 & 13 & 5 & 110.085 & 0.0589628 & 722.689 & 937.925 & $-2.9631303852241762^{\prime} 10^{-6}$ & 0.00869395 & 0.0496483 \\
\hline S 15 & 13 & 6 & 120.521 & 0.0724526 & 547.648 & 1205.92 & $-1.3679984972579015^{\prime} 10^{-7}$ & 0.00848243 & 0.148476 \\
\hline S 15 & 13 & 7 & 275.811 & 0.138378 & 124.35 & 900. & $-9.37278^{\prime} 10^{-7}$ & 0.0141136 & -0.113119 \\
\hline S 15 & 13 & 8 & 198.68 & 0.11904 & 247.753 & 927.909 & 0.0000201012 & 0.00929782 & 0.85427 \\
\hline S 15 & 13 & 9 & 145.249 & 0.0360994 & 348.268 & 589.975 & -0.0000259898 & 0.00578811 & 0.385896 \\
\hline S 15 & 13 & 10 & 130.079 & 0.0286389 & 133.984 & 1207.56 & 0.0000539251 & 0.00451613 & 0.79967 \\
\hline S 15 & 13 & 11 & 64.5145 & 0.0973229 & 412.391 & 1516.28 & -0.0000239566 & 0.00203014 & -2.98299 \\
\hline 15 & 13 & 12 & 107.062 & 0.0967981 & 349.23 & 709.256 & $-8.0266^{\prime} 10^{-7}$ & 0.00309301 & 1.50661 \\
\hline
\end{tabular}

\section{Sample 15 Scan 14:}

\begin{tabular}{|c|c|c|c|c|c|c|c|c|c|c|}
\hline $\mathrm{s}$ & & $\mathrm{T}$ & M & LL & w & $\mathrm{s}$ & $\mathrm{t}$ & a & $\div$ erg & $\mathrm{f}$ \\
\hline & 15 & 14 & 1 & 1855.58 & 0.0216469 & 242.109 & 1130.3 & 0.0000553052 & 0.843637 & -0.437759 \\
\hline $\mathrm{s}$ & 15 & 14 & 2 & 750.733 & 0.00620313 & 550.463 & 1559.36 & $-4.6324^{\prime} 10^{-6}$ & 0.081835 & 0.547688 \\
\hline $\mathrm{s}$ & 15 & 14 & 3 & 245.777 & 0.0236678 & 822.705 & 965.339 & $-4.10849^{\prime} 10^{-6}$ & 0.0158569 & -1.48293 \\
\hline $\mathrm{s}$ & 15 & 14 & 4 & 293.538 & 0.0847815 & 324.426 & 863.706 & -0.0000291712 & 0.0144757 & -1.13601 \\
\hline $\mathrm{s}$ & 15 & 14 & 5 & 95.8503 & 0.059427 & 713.03 & 982.44 & $-3.2896476719047168^{\prime} 10^{-6}$ & 0.00387814 & -1.96352 \\
\hline $\mathrm{s}$ & 15 & 14 & 6 & 145.915 & 0.037366 & 162.505 & 644.672 & -0.0000542464 & 0.00523418 & -1.13415 \\
\hline $\mathrm{s}$ & 15 & 14 & 7 & 105.54 & 0.0172677 & 429.273 & 1473.82 & $-1.8904736309608843^{\prime} 10^{-6}$ & 0.00333694 & 2.96216 \\
\hline $\mathrm{s}$ & 15 & 14 & 8 & 114.107 & 0.0340157 & 379.217 & 1210.73 & $-7.7321^{\prime} 10^{-7}$ & 0.00323284 & 1.2048 \\
\hline $\mathrm{S}$ & 15 & 14 & 9 & 132.473 & 0.0729767 & 141.283 & 1200. & $-1 \cdot 10^{-6}$ & 0.00284288 & 0.905232 \\
\hline s & 15 & 14 & 10 & 287.184 & 0.139079 & 117.928 & 900. & $-1.0067450197803672^{\prime} 10^{-6}$ & 0.00568569 & -0.0011810 \\
\hline $\mathrm{s}$ & 15 & 14 & 11 & 207.983 & 0.119033 & 226.77 & 974.246 & -0.0000194914 & 0.00291336 & 2.15424 \\
\hline & 15 & 14 & 12 & 89.7873 & 0.232739 & 477.033 & 1192.41 & $-9.42253^{\prime} 10^{-6}$ & 0.00131422 & 3.11008 \\
\hline
\end{tabular}

\section{Sample 15 Scan 15:}

\begin{tabular}{llllll}
$S$ & $T$ & $M$ & \multicolumn{1}{c}{ LL } & \multicolumn{1}{c}{ W } \\
$\mathrm{S}$ & 15 & 15 & 1 & 1856.01 & 0.0222672 \\
$\mathrm{~S}$ & 15 & 15 & 2 & 715.116 & 0.00618075 \\
$\mathrm{~S}$ & 15 & 15 & 3 & 196.475 & 0.0281768 \\
$\mathrm{~S}$ & 15 & 15 & 4 & 298.996 & 0.0845263 \\
$\mathrm{~S}$ & 15 & 15 & 5 & 211.746 & 0.0280079 \\
$\mathrm{~S}$ & 15 & 15 & 6 & 122.614 & 0.0248471 \\
$\mathrm{~S}$ & 15 & 15 & 7 & 130.453 & 0.0719938 \\
$\mathrm{~S}$ & 15 & 15 & 8 & 110.213 & 0.0597531 \\
$\mathrm{~S}$ & 15 & 15 & 9 & 130.149 & 0.0347138 \\
$\mathrm{~S}$ & 15 & 15 & 10 & 36.5828 & 0.0000362336 \\
$\mathrm{~S}$ & 15 & 15 & 11 & 304.161 & 0.138732 \\
$\mathrm{~S}$ & 15 & 15 & 12 & 154.72 & 0.123289
\end{tabular}

\begin{tabular}{|c|c|c|c|c|}
\hline $\mathrm{s}$ & $\mathrm{t}$ & a & $\therefore$ erg & $f$ \\
\hline 241.516 & 1125.07 & 0.0000550123 & 0.843704 & -0.326671 \\
\hline 549.033 & 1560.48 & $-4.60352^{\prime} 10^{-6}$ & 0.0791299 & 0.551903 \\
\hline 1568.27 & 1430.86 & $-4.90941^{\prime} 10^{-6}$ & 0.013439 & -0.817157 \\
\hline 27.891 & 864.739 & -0.0000285745 & 0.0159944 & -1.16319 \\
\hline 70.535 & 600.652 & -0.000131265 & 0.00875798 & 0.618219 \\
\hline 3962.22 & 4065.85 & $-1.3989115137416466^{\prime} 10^{-6}$ & 0.0042823 & -1.94018 \\
\hline 9.735 & 1211.71 & $-1.1025474640506084^{\prime} 10^{-6}$ & 0.00401385 & 0.243097 \\
\hline 427.945 & 1337.11 & $4.12063^{\prime} 10^{-6}$ & 0.00300602 & 1.31618 \\
\hline 316.201 & 1113.75 & $-5.05731 \cdot 10^{-6}$ & 0.00314843 & -1.51025 \\
\hline 8.493 & 300.237 & $-1.3828801862349654^{\prime} 10^{-8}$ & 0.000813656 & -1.62221 \\
\hline 3.299 & 900. & $-9.76043^{\prime} 10^{-7}$ & 0.0052202 & 0.0937375 \\
\hline 2.81 & 1031.47 & $-9.7311^{\prime} 10^{-6}$ & 0.00234327 & 1.37624 \\
\hline
\end{tabular}

\section{Sample 15 Scan 16:}

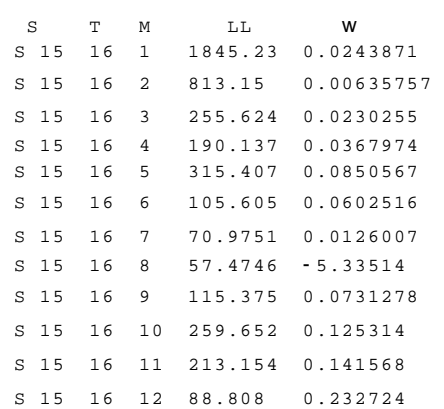

$\begin{array}{llllll}S & 15 & 16 & 12 & 88.808 & 0.232724\end{array}$

$\begin{array}{cl}\mathrm{s} & \mathrm{t} \\ 227.393 & 1111.3 \\ 495.498 & 1559.35 \\ 439.526 & 756.506 \\ 842.758 & 1138.81 \\ 334.658 & 871.214 \\ 737.733 & 1073.39 \\ 575.869 & 857.712 \\ 101899 . & -205742 . \\ 442.887 & 1298.24 \\ 255.492 & 999.992 \\ 193.182 & 900 . \\ 471.239 & 1197.26\end{array}$

$a$
0.0000523781
$-4.82387^{\prime} 10^{-6}$
$3.6047811254304404^{\prime} 10^{-6}$
0.0000124677
-0.0000270319
$-3.5329^{\prime} 10^{-9}$
$2.7502610911447907^{\prime} 10^{-6}$
-0.0000129451
$4.70356^{\prime} 10^{-6}$
$-1.0098180964373598^{\prime} 10^{-6}$
$-1 . ' 10^{-6}$
$-8.85599^{\prime} 10^{-6}$




\section{Sample 15 Scan 17:}

\begin{tabular}{lllllllllll}
$\mathrm{S}$ & $\mathrm{T}$ & $\mathrm{M}$ & \multicolumn{1}{c}{$\mathrm{LL}$} & \multicolumn{1}{c}{$\mathrm{W}$} & $\mathrm{S}$ & $\mathrm{t}$ & \multicolumn{1}{c}{$\mathrm{a}$} & \multicolumn{1}{c}{ erg } & $f$ \\
$\mathrm{~S}$ & 15 & 17 & 1 & 1889.35 & 0.0212639 & 240.969 & 1136.61 & 0.0000545178 & 0.84883 & -0.490957 \\
$\mathrm{~S}$ & 15 & 17 & 2 & 826.69 & 0.00614734 & 554.053 & 1562.78 & $-4.51465^{\prime} 10^{-6}$ & 0.0841718 & 0.556647 \\
$\mathrm{~S}$ & 15 & 17 & 3 & 135.809 & 0.0273895 & 221.709 & 1527.97 & 0.0000183226 & 0.00825876 & 2.56341 \\
$\mathrm{~S}$ & 15 & 17 & 4 & 338.265 & 0.0228937 & 373.006 & 730.044 & $4.50886^{\prime} 10^{-6}$ & 0.0162943 & -2.7258 \\
$\mathrm{~S}$ & 15 & 17 & 5 & 297.322 & 0.0827484 & 316.079 & 817.086 & -0.0000314426 & 0.0104116 & 2.89063 \\
$\mathrm{~S}$ & 15 & 17 & 6 & 213.997 & 0.0611241 & 478.426 & 874.79 & -0.000031236 & 0.00579291 & -0.89311 \\
$\mathrm{~S}$ & 15 & 17 & 7 & 130.673 & -0.773146 & 84711.4 & -14254.2 & -0.0000265253 & 0.00269293 & 0.29315 \\
$\mathrm{~S}$ & 15 & 17 & 8 & 231.38 & 0.123572 & 223.178 & 1000. & $-1.0^{-6}$ & 0.0035854 & -0.856643 \\
$\mathrm{~S}$ & 15 & 17 & 9 & 61.2238 & 0.0949218 & 542.202 & 860.095 & $2.25526^{\prime} 10^{-6}$ & 0.00104015 & 0.0948512 \\
$\mathrm{~S}$ & 15 & 17 & 10 & 298.022 & 0.142307 & 169.214 & 900. & $-9.76041^{\prime} 10^{-7}$ & 0.00291055 & 0.244028 \\
$\mathrm{~S}$ & 15 & 17 & 11 & 119.778 & 0.035441 & 369.203 & 712.992 & -0.000035765 & 0.00153114 & 2.11608 \\
$\mathrm{~S}$ & 15 & 17 & 12 & 94.7471 & 0.232835 & 488.474 & 1193.04 & $-8.54586^{\prime} 10^{-6}$ & 0.00108708 & 3.0283
\end{tabular}

Sample 15 Scan 18:

\begin{tabular}{llllll}
$S$ & $T$ & $M$ & \multicolumn{1}{c}{ LL } & \multicolumn{1}{c}{ W } \\
$S$ & 15 & 18 & 1 & 1872.52 & 0.0215212 \\
$S$ & 15 & 18 & 2 & 778.167 & 0.0061368 \\
$S$ & 15 & 18 & 3 & 124.64 & 0.0277401 \\
$S$ & 15 & 18 & 4 & 304.224 & 0.0228976 \\
$S$ & 15 & 18 & 5 & 302.047 & 0.0842052 \\
$S$ & 15 & 18 & 6 & 109.186 & 0.0593602 \\
$S$ & 15 & 18 & 7 & 118.136 & 0.0419807 \\
$S$ & 15 & 18 & 8 & 96.0861 & 0.0732097 \\
$S$ & 15 & 18 & 9 & 328.819 & 0.139632 \\
$S$ & 15 & 18 & 10 & 79.1923 & 0.00573747 \\
$S$ & 15 & 18 & 11 & 175.894 & 0.118576 \\
$S$ & 15 & 18 & 12 & 97.3527 & 0.232881
\end{tabular}

\begin{tabular}{|c|c|c|c|c|}
\hline $\mathrm{s}$ & $\mathrm{t}$ & a & $\frac{\circ}{6}$ erg & $f$ \\
\hline 1.42 & 1133.21 & 0.0000545583 & 0.846265 & -0.44803 \\
\hline 2.277 & 1561.59 & $-4.5054815543121555^{\prime} 10^{-6}$ & 0.0823077 & 0.559851 \\
\hline 3.489 & 1520.83 & 0.000019003 & 0.00814 & 2.71007 \\
\hline 5.16 & 728.545 & $4.8199858238328345^{\prime} 10^{-6}$ & 0.0160851 & -2.74329 \\
\hline 5.795 & 848.946 & -0.0000305745 & 0.0117932 & 0.186287 \\
\hline 8.089 & 980.038 & $-3.4362864648379634^{\prime} 10^{-6}$ & 0.00345932 & -1.68589 \\
\hline .456 & 723.242 & $-1.0047943373801873^{\prime} 10^{-6}$ & 0.00334072 & 1.29898 \\
\hline .84 & 1193.28 & $2.18696^{\prime} 10^{-6}$ & 0.00239366 & 1.42974 \\
\hline 375 & 900. & $-9.99908^{\prime} 10^{-7}$ & 0.00508683 & -0.138396 \\
\hline 383 & 359.237 & -0.0000114553 & 0.00145907 & 3.0794 \\
\hline 65 & 940.056 & 0.0000108931 & 0.00282384 & -0.512574 \\
\hline & & $.47105^{\prime}$ & 9 & 0.8912 \\
\hline
\end{tabular}

\section{Sample 15 Scan 19:}

\begin{tabular}{llllll}
$S$ & $T$ & $M$ & $L L$ & \multicolumn{1}{c}{$W$} \\
$S$ & 15 & 19 & 1 & 1882.88 & 0.0218605 \\
$S$ & 15 & 19 & 2 & 771.383 & 0.00615622 \\
$S$ & 15 & 19 & 3 & 252.604 & 0.0234077 \\
$S$ & 15 & 19 & 4 & 275.078 & 0.0834344 \\
$S$ & 15 & 19 & 5 & 105.319 & 0.0595366 \\
$S$ & 15 & 19 & 6 & 168.992 & 0.0372217 \\
$S$ & 15 & 19 & 7 & 122.976 & 0.0170087 \\
$S$ & 15 & 19 & 8 & 145.742 & 0.0340193 \\
$S$ & 15 & 19 & 9 & 145.759 & 0.0727166 \\
$S$ & 15 & 19 & 10 & 375.924 & 0.139645 \\
$S$ & 15 & 19 & 11 & 159.537 & 0.119655 \\
$S$ & 15 & 19 & 12 & 174.415 & 0.00841692
\end{tabular}

\begin{tabular}{|c|c|c|}
\hline $\mathrm{s}$ & $t$ & a \\
\hline 241.299 & 1129.91 & 0.0000548202 \\
\hline 550.543 & 1562.45 & $-4.53999^{\prime} 10^{-6}$ \\
\hline 805.214 & 939.608 & $-4.04887^{\prime} 10^{-6}$ \\
\hline 348.123 & 842.714 & -0.0000297436 \\
\hline 594.717 & 1005.56 & $-3.06976^{\prime} 10^{-6}$ \\
\hline 57.059 & 639.666 & -0.0000528638 \\
\hline 413.761 & 1491.5 & $-2.5386^{\prime} 10^{-6}$ \\
\hline 5.441 & 1219.87 & $-6.88796^{\prime} 10^{-7}$ \\
\hline 3.132 & 1200 & $-1.0000009788210643^{\prime} 10^{-6}$ \\
\hline 0.384 & 900. & $-9.13321^{\prime} 10^{-7}$ \\
\hline .221 & 903.218 & 0.0000141753 \\
\hline 174 & 16 & -0.0000577048 \\
\hline
\end{tabular}

\begin{tabular}{ll}
\multicolumn{1}{c}{$\quad \mathrm{c}$ erg } & \multicolumn{1}{c}{$f$} \\
0.847849 & -0.397789 \\
0.0809862 & 0.553931 \\
0.0154469 & -0.864738 \\
0.012925 & 0.7892 \\
0.00408049 & 3.02253 \\
0.00571242 & -0.971127 \\
0.00359007 & 2.70185 \\
0.00372071 & 0.904184 \\
0.00272706 & 1.01993 \\
0.00431002 & -0.0598138 \\
0.00245925 & -2.36213 \\
0.00227562 & -1.78783
\end{tabular}

Sample 15 Scan 20:

\begin{tabular}{|c|c|c|c|c|c|c|c|c|c|c|}
\hline $\mathrm{s}$ & & $\mathrm{T}$ & M & LL & w & $\mathrm{s}$ & $\mathrm{t}$ & a & $\frac{\circ}{6}$ erg & $f$ \\
\hline $\mathrm{s}$ & 15 & 20 & 1 & 1780.48 & 0.0227394 & 243.057 & 1118.66 & 0.0000550458 & 0.831443 & -0.243293 \\
\hline & 15 & 20 & 2 & 598.088 & 0.00615213 & 543.074 & 1556.75 & $-4.63397^{\prime} 10^{-6}$ & 0.0752392 & 0.573199 \\
\hline & 15 & 20 & 3 & 171.781 & 0.0233962 & 824.274 & 940.395 & $-4.21914^{\prime} 10^{-6}$ & 0.0144365 & -0.93025 \\
\hline & 15 & 20 & 4 & 434.472 & 0.088955 & 223.473 & 934.789 & -0.0000302963 & 0.0219288 & -0.927362 \\
\hline & 15 & 20 & 5 & 145.968 & 0.0585692 & 740.524 & 901.747 & $-3.13955^{\prime} 10^{-6}$ & 0.0074824 & 2.52589 \\
\hline & 15 & 20 & 6 & 141.848 & 0.07207 & 772.534 & 1226.52 & $-1.1528154661430103^{\prime} 10^{-6}$ & 0.00629631 & -0.917797 \\
\hline & 15 & 20 & 7 & 169.017 & 0.0365521 & 363.249 & 598.047 & -0.0000259467 & 0.00642453 & 0.243825 \\
\hline$S$ & 15 & 20 & 8 & 182.517 & 0.0340647 & 111.537 & 1213.4 & 0.000595987 & 0.00582055 & 0.149061 \\
\hline s & 15 & 20 & 9 & 392.201 & 0.140963 & 97.0214 & 899.999 & $-1 .^{\prime} 10^{-6}$ & 0.00647793 & 0.153659 \\
\hline S & 15 & 20 & 10 & 193.86 & 0.117876 & 206.856 & 901.026 & 0.0000122469 & 0.00372845 & -1.83762 \\
\hline & 15 & 20 & 11 & 131.697 & 0.145397 & 5634.84 & 15611.8 & $-5.88154^{\prime} 10^{-6}$ & 0.00231222 & 2.83674 \\
\hline & 15 & 20 & 12 & 68.5929 & 0.0341323 & 1485.11 & 1593.86 & $2.64094^{\prime} 10^{-7}$ & 0.00108892 & 0.487782 \\
\hline
\end{tabular}


Sample 15 Scan 21:

\begin{tabular}{|c|c|c|c|c|c|c|c|c|c|c|}
\hline & s & $\mathrm{T}$ & M & LL & w & $\mathrm{s}$ & $\mathrm{t}$ & a & $\div$ erg & f \\
\hline $\mathrm{s}$ & 15 & 21 & 1 & 1450.76 & 0.0238849 & 244.367 & 1102.39 & 0.0000550544 & 0.765609 & -0.028866 \\
\hline $\mathrm{s}$ & 15 & 21 & 2 & 323.466 & 0.00600688 & 534.677 & 1535.73 & $-4.68146^{\prime} 10^{-6}$ & 0.0644315 & 0.66723 \\
\hline $\mathrm{s}$ & 15 & 21 & 3 & 407.469 & 0.0888951 & 232.833 & 978.575 & -0.0000191155 & 0.0422952 & 0.873986 \\
\hline $\mathrm{s}$ & 15 & 21 & 4 & 110.369 & 0.071925 & 732.149 & 1137.69 & $-6.32995^{\prime} 10^{-7}$ & 0.0131749 & -1.35204 \\
\hline s & 15 & 21 & 5 & 95.7715 & 0.0587389 & 740.308 & 929.4 & $-2.80847^{\prime} 10^{-6}$ & 0.0103114 & 0.431219 \\
\hline $\mathrm{s}$ & 15 & 21 & 6 & 72.7451 & 0.03041 & 302.432 & 1449.33 & 0.0000179246 & 0.00719817 & -1.79031 \\
\hline s & 15 & 21 & 7 & 156.937 & 0.0228976 & 410.934 & 726.908 & $5.9625^{\prime} 10^{-6}$ & 0.0138554 & -3.02124 \\
\hline $\mathrm{s}$ & 15 & 21 & 8 & 324.109 & 0.139257 & 136.234 & 900. & $-1.0003435969165067^{\prime} 10^{-6}$ & 0.0209688 & 0.0564061 \\
\hline $\mathrm{s}$ & 15 & 21 & 9 & 450.641 & 0.12346 & 175.552 & 986.823 & $-7.89337^{\prime} 10^{-6}$ & 0.0134782 & 0.409211 \\
\hline s & 15 & 21 & 10 & 92.904 & 0.232497 & 450.699 & 1204.26 & $-5.7025353052541265^{\prime} 10^{-6}$ & 0.00417802 & 0.377602 \\
\hline $\mathrm{s}$ & 15 & 21 & 11 & 91.1162 & 0.0991508 & 743.129 & 956.781 & $-1.8424^{\prime} 10^{-6}$ & 0.00373737 & 2.18336 \\
\hline & 15 & 21 & 12 & 103.367 & .13161 & 85.0223 & 813.396 & $-3.6540696669827117^{\prime} 10^{-6}$ & 0.0036557 & 1.80186 \\
\hline
\end{tabular}

Sample 15 Scan 22:

\begin{tabular}{|c|c|c|c|c|c|c|c|c|c|c|}
\hline$s$ & S & $\mathrm{T}$ & $\mathrm{M}$ & LL & w & $\mathrm{s}$ & $t$ & a & $\frac{\circ}{\partial}$ erg & $f$ \\
\hline $\mathrm{s}$ & 15 & 22 & 1 & 1555.7 & 0.0235444 & 245.51 & 1107.29 & 0.000054753 & 0.788959 & -0.0844464 \\
\hline$S$ & 15 & 22 & 2 & 393.349 & 0.00604481 & 538.714 & 1544.47 & $-4.63294^{\prime} 10^{-6}$ & 0.068186 & 0.635348 \\
\hline $\mathrm{s}$ & 15 & 22 & 3 & 398.48 & 0.0905588 & 154.627 & 963.691 & -0.0000331027 & 0.03577 & 2.47811 \\
\hline$S$ & 15 & 22 & 4 & 144.718 & 0.0719653 & 729.017 & 1099.47 & $-9.07941^{\prime} 10^{-7}$ & 0.0141921 & 1.64383 \\
\hline $\mathrm{s}$ & 15 & 22 & 5 & 88.6436 & 0.0303119 & 336.318 & 1447.18 & 0.0000184214 & 0.00773144 & -1.66759 \\
\hline $\mathrm{S}$ & 15 & 22 & 6 & 117.642 & 0.0586971 & 759.117 & 936.604 & $-3.08562^{\prime} 10^{-6}$ & 0.00925735 & 0.161397 \\
\hline $\mathrm{s}$ & 15 & 22 & 7 & 216.689 & 0.0229386 & 405.277 & 724.081 & $5.9150465669250555^{\prime} 10^{-6}$ & 0.0144465 & -2.90722 \\
\hline $\mathrm{S}$ & 15 & 22 & 8 & 490.953 & 0.124727 & 155.885 & 1000 . & $-1 \cdot 10^{-6}$ & 0.0171662 & -0.825234 \\
\hline $\mathrm{s}$ & 15 & 22 & 9 & 72.6543 & 0.232877 & 464.158 & 1237.59 & $-4.39157^{\prime} 10^{-6}$ & 0.00298114 & -1.13592 \\
\hline $\mathrm{S}$ & 15 & 22 & 10 & 90.2869 & 0.0444258 & 214.389 & 800. & $-1.0000654150856763^{\prime} 10^{-6}$ & 0.00341246 & -2.53596 \\
\hline $\mathrm{s}$ & 15 & 22 & 11 & 164.115 & 0.144799 & 231.694 & 1000 . & $-1.0161417388256785^{\prime} 10^{-6}$ & 0.00545358 & -1.51412 \\
\hline$S$ & 15 & 22 & 12 & 101.129 & 0.0850918 & 172.848 & 1300 . & $-1.0000687246729552^{\prime} 10^{-6}$ & 0.00198854 & -2.34079 \\
\hline
\end{tabular}

Sample 15 Scan 23:

\begin{tabular}{llllll}
$S$ & $T$ & $M$ & \multicolumn{1}{c}{ LL } & \multicolumn{1}{c}{ W } \\
$\mathrm{S}$ & 15 & 23 & 1 & 1441.54 & 0.0238364 \\
$\mathrm{~S}$ & 15 & 23 & 2 & 320.44 & 0.00601217 \\
$\mathrm{~S}$ & 15 & 23 & 3 & 624.665 & 0.0897493 \\
$\mathrm{~S}$ & 15 & 23 & 4 & 102.336 & 0.0719041 \\
$\mathrm{~S}$ & 15 & 23 & 5 & 97.5247 & 0.0587248 \\
$\mathrm{~S}$ & 15 & 23 & 6 & 73.0054 & 0.0302988 \\
$\mathrm{~S}$ & 15 & 23 & 7 & 155.402 & 0.0229671 \\
$\mathrm{~S}$ & 15 & 23 & 8 & 386.96 & 0.123813 \\
$\mathrm{~S}$ & 15 & 23 & 9 & 261.621 & 0.140857 \\
$\mathrm{~S}$ & 15 & 23 & 10 & 93.2594 & 0.232763 \\
$\mathrm{~S}$ & 15 & 23 & 11 & 146.215 & 0.111426 \\
$\mathrm{~S}$ & 15 & 23 & 12 & 106.434 & 0.0431602
\end{tabular}

\begin{tabular}{|c|c|c|c|c|}
\hline s & $t$ & a & $\therefore$ erg & $f$ \\
\hline 45.585 & 1103.08 & 0.0000548649 & 0.763437 & -0.0312969 \\
\hline 538.245 & 1540.42 & $-4.6343946614950535^{\prime} 10^{-6}$ & 0.0645046 & 0.650743 \\
\hline 246.387 & 984.379 & -0.0000208265 & 0.0427164 & 0.373398 \\
\hline 88.429 & 1190.28 & $-7.2345^{\prime} 10^{-7}$ & 0.0124215 & 1.19678 \\
\hline 46.382 & 925.361 & $-2.7371571329135256^{\prime} 10^{-6}$ & 0.0107114 & 0.67615 \\
\hline 01.926 & 1451.45 & 0.0000191369 & 0.0073617 & -1.85698 \\
\hline 405.348 & 720.351 & $5.8959561424202345^{\prime} 10^{-6}$ & 0.0139901 & -2.86128 \\
\hline 250.022 & 1000 & $-1 \cdot 10^{-6}$ & 0.0199754 & -0.895956 \\
\hline 236.856 & 900. & $-1 \cdot 10^{-6}$ & 0.0143779 & 0.330369 \\
\hline 438.918 & 1234.73 & $-4.76006^{\prime} 10^{-6}$ & 0.00435046 & -0.254735 \\
\hline 231.86 & 1100 & $-1 \cdot 10^{-6}$ & 0.00385501 & 0.70097 \\
\hline 96.711 & 710.202 & $-9.68735^{\prime} 10^{-7}$ & 0.00410283 & 1.37535 \\
\hline
\end{tabular}

\section{Sample 15 Scan 24:}

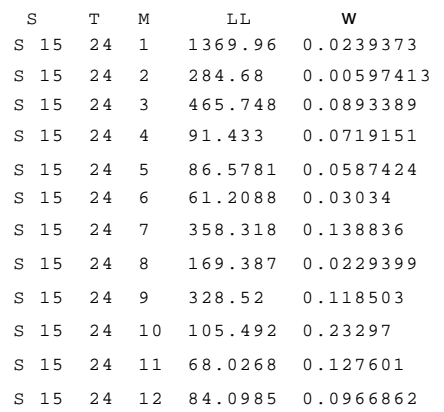

$\begin{array}{llllll}S & 15 & 24 & 12 & 84.0985 & 0.0966862\end{array}$

\begin{tabular}{|c|c|c|c|c|}
\hline $\mathrm{s}$ & $t$ & a & $\frac{\circ}{8}$ erg & $f$ \\
\hline 245.607 & 1100.79 & 0.0000548382 & 0.745884 & -0.0102611 \\
\hline 536.926 & 1535.24 & $-4.64628 \cdot 10^{-6}$ & 0.0626474 & 0.673453 \\
\hline 243.701 & 978.969 & -0.0000206206 & 0.0454704 & 0.728577 \\
\hline 759.549 & 1183.1 & $-5.44979^{\prime} 10^{-7}$ & 0.0126191 & 1.60048 \\
\hline 747.08 & 924.368 & $-2.63005 \times 10^{-6}$ & 0.0109311 & 0.628868 \\
\hline 299.546 & 1448.36 & 0.0000189187 & 0.00717643 & -1.80517 \\
\hline 135.661 & 900. & $-9.39551^{\prime} 10^{-7}$ & 0.0248921 & -0.101069 \\
\hline 402.424 & 718.259 & $5.7365695355950995^{\prime} 10^{-6}$ & 0.0138368 & -2.8398 \\
\hline 179. & 1000 & $-1.0389^{\prime} 10^{-6}$ & 0.0183567 & -1.18207 \\
\hline 438.772 & 1246.93 & $-5.70998^{\prime} 10^{-6}$ & 0.00566746 & 3.13191 \\
\hline 60.755 & 700. & $-1 \cdot 10^{-6}$ & 0.00232575 & -2.33159 \\
\hline 07.162 & 617.955 & $-1.5533607744838807^{\prime} 10^{-6}$ & 0.00392121 & -2.29364 \\
\hline
\end{tabular}




\section{Sample 15 Scan 25:}

\begin{tabular}{|c|c|c|c|c|c|c|c|c|c|}
\hline $\mathrm{s}$ & $\mathrm{T}$ & M & LL & w & $\mathrm{s}$ & $t$ & a & $\frac{\circ}{6}$ erg & $f$ \\
\hline S 15 & 25 & 1 & 1372.62 & 0.0238696 & 245.58 & 1101.27 & 0.0000547701 & 0.746558 & -0.0219432 \\
\hline 15 & 25 & 2 & 291.19 & 0.0060082 & 537.173 & 1536.9 & $-4.6928851256837865^{\prime} 10^{-6}$ & 0.0637067 & 0.664334 \\
\hline S 15 & 25 & 3 & 386.805 & 0.090019 & 207.896 & 990.291 & -0.0000206879 & 0.0458046 & -0.313239 \\
\hline 15 & 25 & 4 & 109.069 & 0.0718776 & 747.788 & 1122.84 & $-3.2676216776753744^{\prime} 10^{-7}$ & 0.014708 & -0.390392 \\
\hline 15 & 25 & 5 & 90.3149 & 0.0587098 & 742.6 & 925.104 & $-2.70087^{\prime} 10^{-6}$ & 0.0110217 & 0.601154 \\
\hline S 15 & 25 & 6 & 63.8305 & 0.0301648 & 296.375 & 1451.4 & 0.0000190052 & 0.00721057 & -1.89509 \\
\hline 15 & 25 & 7 & 276.519 & 0.138699 & 121.275 & 900. & $-9.61551^{\prime} 10^{-7}$ & 0.0238653 & -0.14501 \\
\hline 15 & 25 & 8 & 230.138 & 0.118504 & 176.561 & 999.999 & $-1.0092606138046703^{\prime} 10^{-6}$ & 0.0163399 & -1.29098 \\
\hline S 15 & 25 & 9 & 226.298 & 0.0229557 & 403.599 & 718.937 & $5.8467703543549164^{\prime} 10^{-6}$ & 0.0140128 & -2.86024 \\
\hline S 15 & 25 & 10 & 154.073 & 0.217435 & 404.663 & 999.526 & -0.0000363011 & 0.00787908 & -2.83539 \\
\hline 15 & 25 & 11 & 61.7661 & 0.127386 & 197.735 & 711.016 & $-1.00722^{\prime} 10^{-6}$ & 0.00283213 & 2.34651 \\
\hline 15 & 25 & 12 & 96.982 & 0.0434507 & 199.247 & 711.129 & $-9.6803^{\prime} 10^{-7}$ & 0.00410937 & 1.27346 \\
\hline
\end{tabular}

Sample 15 Scan 26:

\begin{tabular}{|c|c|c|c|c|c|c|c|c|c|c|}
\hline s & & $\mathrm{T}$ & M & LL & w & s & $\mathrm{t}$ & a & $\frac{\circ}{6}$ erg & $f$ \\
\hline $\mathrm{s}$ & 15 & 26 & 1 & 1243.67 & 0.024013 & 245.616 & 1097.69 & 0.0000548553 & 0.711675 & 0.00444241 \\
\hline s & 15 & 26 & 2 & 234.005 & 0.00604747 & 534.965 & 1538.51 & $-4.68376^{\prime} 10^{-6}$ & 0.0599113 & 0.637967 \\
\hline $\mathrm{s}$ & 15 & 26 & 3 & 355.283 & 0.0912486 & 252.358 & 1009.36 & -0.0000215584 & 0.0493577 & -2.23553 \\
\hline $\mathrm{s}$ & 15 & 26 & 4 & 77.5631 & 0.0718148 & 788.284 & 1171.69 & $-3.9217446264145745^{\prime} 10^{-7}$ & 0.0132503 & 2.22492 \\
\hline $\mathrm{s}$ & 15 & 26 & 5 & 69.8921 & 0.0587383 & 758.621 & 922.83 & $-2.4376859516132255^{\prime} 10^{-6}$ & 0.0110907 & 0.556236 \\
\hline $\mathrm{s}$ & 15 & 26 & 6 & 266.396 & 0.138937 & 206.638 & 900.108 & $-1 \cdot^{\prime} 10^{-6}$ & 0.0296108 & -0.214208 \\
\hline s & 15 & 26 & 7 & 203.581 & 0.120811 & 179.049 & 999.998 & $-8.98829^{\prime} 10^{-7}$ & 0.0219839 & -1.38089 \\
\hline $\mathrm{s}$ & 15 & 26 & 8 & 68.2171 & 0.0302467 & 296.185 & 1449.8 & 0.0000193849 & 0.00670024 & -1.91386 \\
\hline $\mathrm{s}$ & 15 & 26 & 9 & 150.702 & 0.0229938 & 384.576 & 705.208 & $5.54939^{\prime} 10^{-6}$ & 0.0132771 & -2.58066 \\
\hline $\mathrm{s}$ & 15 & 26 & 10 & 109.292 & 0.233729 & 431.766 & 1289.31 & $-6.37333^{\prime} 10^{-6}$ & 0.00845126 & -0.714654 \\
\hline s & 15 & 26 & 11 & 212.397 & 0.20792 & 155.931 & 788.969 & -0.0000145343 & 0.00863885 & -2.78278 \\
\hline$S$ & 15 & 26 & 12 & 65.1817 & 0.0995592 & 710.5 & 900.928 & $-8.48354^{\prime} 10^{-7}$ & 0.00407264 & 0.809748 \\
\hline
\end{tabular}

\section{Sample 15 Scan 27:}

\begin{tabular}{|c|c|c|c|c|c|c|c|c|c|}
\hline $\mathrm{s}$ & $\mathrm{T}$ & M & LL & w & $\mathrm{s}$ & $\mathrm{t}$ & a & $\frac{\circ}{6}$ erg & $f$ \\
\hline S 15 & 27 & 1 & 1313.03 & 0.0237923 & 246.234 & 1100.12 & 0.0000548117 & 0.730997 & -0.0349407 \\
\hline S 15 & 27 & 2 & 264.825 & 0.00604787 & 537.712 & 1540.87 & $-4.64551 \cdot 10^{-6}$ & 0.062301 & 0.628801 \\
\hline S 15 & 27 & 3 & 321.921 & 0.0912495 & 130.952 & 1010.3 & -0.0000212173 & 0.0436812 & -2.34203 \\
\hline S 15 & 27 & 4 & 142.671 & 0.0720455 & 605.788 & 1000.27 & $8.92523^{\prime} 10^{-7}$ & 0.0214657 & 1.74216 \\
\hline S 15 & 27 & 5 & 85.6059 & 0.0585596 & 672.909 & 892.652 & $-3.30733^{\prime} 10^{-6}$ & 0.0114844 & 2.47117 \\
\hline S 15 & 27 & 6 & 283.071 & 0.13879 & 145.741 & 900.276 & $-1 \cdot 10^{-6}$ & 0.0263738 & -0.28075 \\
\hline S 15 & 27 & 7 & 174.425 & 0.122475 & 264.319 & 1000.01 & $-9.99914^{\prime} 10^{-7}$ & 0.015286 & -1.63019 \\
\hline S 15 & 27 & 8 & 83.9172 & 0.0299559 & 292.156 & 1456.82 & 0.0000199451 & 0.00698947 & -2.12111 \\
\hline S 15 & 27 & 9 & 191.111 & 0.0230103 & 379.011 & 702.694 & $5.316^{\prime} 10^{-6}$ & 0.0138895 & -2.49728 \\
\hline S 15 & 27 & 10 & 118.127 & 0.23382 & 416.893 & 1303.86 & $-6.90411 \times 10^{-6}$ & 0.00734843 & 2.19287 \\
\hline 15 & 27 & 11 & 44.6862 & 0.0720219 & 4938.9 & 8447.96 & $4.4596652174782484^{\prime} 10^{-7}$ & 0.00256122 & -1.33377 \\
\hline 15 & 27 & 12 & 147.315 & 0.208943 & 212.1 & 830.98 & -0.0000192656 & 0.00650946 & 1.13344 \\
\hline
\end{tabular}

\section{Sample 15 Scan 28:}

\begin{tabular}{|c|c|c|c|c|c|c|c|c|c|c|}
\hline s & & $\mathrm{T}$ & M & LL & w & s & $\mathrm{t}$ & a & $\frac{\circ}{\partial} \operatorname{erg}$ & $f$ \\
\hline $\mathrm{s}$ & 15 & 28 & 1 & 1158.33 & 0.0239919 & 246.962 & 1095.87 & 0.0000548554 & 0.68599 & 0.00315327 \\
\hline s & 15 & 28 & 2 & 204.902 & 0.00605286 & 535.957 & 1538.39 & $-4.65302^{\prime} 10^{-6}$ & 0.0579651 & 0.628628 \\
\hline $\mathrm{s}$ & 15 & 28 & 3 & 448.119 & 0.0897842 & 235.333 & 948.352 & -0.000025804 & 0.0550966 & -3.07507 \\
\hline $\mathrm{s}$ & 15 & 28 & 4 & 321.966 & 0.138647 & 184.182 & 900. & $-9.31659^{\prime} 10^{-7}$ & 0.0336833 & -0.417473 \\
\hline s & 15 & 28 & 5 & 159.229 & 0.120859 & 199.787 & 1000 . & $-1.0005353292880292^{\prime} 10^{-6}$ & 0.0242547 & -1.65023 \\
\hline s & 15 & 28 & 6 & 77.8498 & 0.0720282 & 795.759 & 1303.35 & $-6.73306^{\prime} 10^{-7}$ & 0.0106011 & -0.966466 \\
\hline $\mathrm{s}$ & 15 & 28 & 7 & 87.2456 & 0.058797 & 779.17 & 916.826 & $-2.208811^{\prime} 10^{-6}$ & 0.0109399 & 0.75595 \\
\hline $\mathrm{s}$ & 15 & 28 & 8 & 56.3833 & 0.0303105 & 294.684 & 1447.26 & 0.0000207629 & 0.00657887 & -1.91473 \\
\hline $\mathrm{s}$ & 15 & 28 & 9 & 133.386 & 0.234034 & 410.497 & 1311.91 & $-7.13794^{\prime} 10^{-6}$ & 0.0141633 & 0.0849816 \\
\hline s & 15 & 28 & 10 & 139.861 & 0.0231114 & 372.999 & 694.615 & $5.46239^{\prime} 10^{-6}$ & 0.0129554 & -2.37987 \\
\hline $\mathrm{s}$ & 15 & 28 & 11 & 120.809 & 0.213086 & 487.688 & 1016.98 & $-4.68883^{\prime} 10^{-6}$ & 0.00982104 & -1.10002 \\
\hline s & 15 & 28 & 12 & 73.2371 & 0.122747 & 1135.09 & 1436.18 & -0.0000122525 & 0.00540116 & -0.864567 \\
\hline
\end{tabular}




\section{Sample 15 Scan 29:}

\begin{tabular}{|c|c|c|c|c|c|c|c|c|c|c|}
\hline s & & $\mathrm{T}$ & M & LL & w & $\mathrm{s}$ & $\mathrm{t}$ & a & $\%$ erg & $f$ \\
\hline & 15 & 29 & 1 & 1166.55 & 0.023751 & 246.998 & 1097.43 & 0.0000552473 & 0.68856 & -0.0531369 \\
\hline 5 & 15 & 29 & 2 & 211.609 & 0.00605562 & 536.943 & 1535.17 & $-4.70331 \cdot 10^{-6}$ & 0.0591836 & 0.632545 \\
\hline & 15 & 29 & 3 & 225.38 & 0.089641 & 189.985 & 1000 . & $-1.0044774077339316^{\prime} 10^{-6}$ & 0.0490879 & -1.74805 \\
\hline & 15 & 29 & 4 & 82.1699 & 0.0718681 & 721.388 & 1046.19 & $9.28931^{\prime} 10^{-7}$ & 0.0159142 & -1.76714 \\
\hline & 15 & 29 & 5 & 236.022 & 0.12479 & 196.808 & 999.446 & $-9.99257^{\prime} 10^{-7}$ & 0.0387668 & -1.48257 \\
\hline & 15 & 29 & 6 & 74.6553 & 0.0586887 & 761.762 & 904.17 & $-2.1231249572363873^{\prime} 10^{-6}$ & 0.0105789 & 1.46274 \\
\hline$S$ & 15 & 29 & 7 & 52.0714 & 0.0303071 & 292.052 & 1448.72 & 0.0000215706 & 0.00692484 & -1.99838 \\
\hline 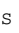 & 15 & 29 & 8 & 200.081 & 0.14152 & 153.498 & 900.001 & $-9.76042^{\prime} 10^{-7}$ & 0.01906 & -0.168764 \\
\hline & 15 & 29 & 9 & 128.246 & 0.233878 & 417.427 & 1299.05 & $-6.28963^{\prime} 10^{-6}$ & 0.0132995 & 3.13772 \\
\hline & 15 & 29 & 10 & 147.632 & 0.0231902 & 360.735 & 687.548 & $5.29246^{\prime} 10^{-6}$ & 0.0133402 & -2.20594 \\
\hline$S$ & 15 & 29 & 11 & 267.887 & 0.20912 & 209.567 & 832.756 & -0.0000121186 & 0.0118672 & 0.543621 \\
\hline & 15 & 29 & 12 & 93.1691 & 0.125937 & 663.317 & 1063.16 & $4.84413^{\prime} 10^{-6}$ & 0.00640407 & 0.563364 \\
\hline
\end{tabular}

\section{Sample 15 Scan 30:}

\begin{tabular}{llllll}
\multicolumn{2}{c}{$S$} & T & M & \multicolumn{1}{c}{ LL } & \multicolumn{1}{c}{ W } \\
S & 15 & 30 & 1 & 1484.1 & 0.0229635 \\
$S$ & 15 & 30 & 2 & 371.259 & 0.00606387 \\
$S$ & 15 & 30 & 3 & 274.113 & 0.0878356 \\
$S$ & 15 & 30 & 4 & 132.525 & 0.0233153 \\
$S$ & 15 & 30 & 5 & 95.4443 & 0.0587927 \\
$S$ & 15 & 30 & 6 & 225.455 & 0.124292 \\
$S$ & 15 & 30 & 7 & 99.0791 & 0.0720492 \\
$S$ & 15 & 30 & 8 & 222.719 & 0.140142 \\
$S$ & 15 & 30 & 9 & 123.323 & 0.0407442 \\
$S$ & 15 & 30 & 10 & 83.0376 & 0.0319986 \\
$S$ & 15 & 30 & 11 & 83.4928 & 0.0858694 \\
$S$ & 15 & 30 & 12 & 103.688 & 0.234315
\end{tabular}

\begin{tabular}{|c|c|c|c|c|}
\hline $\mathrm{s}$ & $\mathrm{t}$ & a & $\frac{\circ}{\partial} \operatorname{erg}$ & $50-1=1$ \\
\hline 46.903 & 1109.07 & 0.0000550661 & 0.773293 & -0.195134 \\
\hline 544.273 & 1543.45 & $-4.6673^{\prime} 10^{-6}$ & 0.0699015 & 0.61822 \\
\hline 297.578 & 917.446 & -0.0000267891 & 0.0371917 & 0.0142817 \\
\hline 804.727 & 914.117 & $-3.98945^{\prime} 10^{-6}$ & 0.0146377 & -0.51683 \\
\hline 748.852 & 922.708 & $-2.7118954397889327^{\prime} 10^{-6}$ & 0.00940245 & 0.790356 \\
\hline 254.607 & 999.962 & $-1.0032094931994964^{\prime} 10^{-6}$ & 0.0167585 & -1.20074 \\
\hline 581.319 & 1396.8 & $-4.19009^{\prime} 10^{-7}$ & 0.00727535 & -1.12372 \\
\hline 190.25 & 900. & $-1.00003^{\prime} 10^{-6}$ & 0.0107167 & 0.145592 \\
\hline 387.142 & 662.96 & -0.0000251971 & 0.00686097 & -2.60098 \\
\hline 675.415 & 1167.33 & $-3.7713475688520774^{\prime} 10^{-6}$ & 0.00416539 & 2.25835 \\
\hline 394.127 & 1235.32 & -0.0000119512 & 0.00385361 & -3.02628 \\
\hline 8.942 & 316.19 & $-6.47572^{\prime} 10^{-6}$ & 0.00435884 & 0.619 \\
\hline
\end{tabular}

\section{Sample 15 Scan 31:}

\begin{tabular}{|c|c|c|c|c|c|c|c|c|c|}
\hline $\mathrm{s}$ & $\mathrm{T}$ & M & LL & w & $\mathrm{s}$ & $\mathrm{t}$ & a & $\frac{\circ}{\partial}$ erg & $f$ \\
\hline S 15 & 31 & 1 & 1482.6 & 0.0228062 & 245.928 & 1110.11 & 0.0000553478 & 0.772955 & -0.233115 \\
\hline S 15 & 31 & 2 & 375.669 & 0.00605507 & 539.771 & 1538.08 & $-4.75368^{\prime} 10^{-6}$ & 0.070699 & 0.640839 \\
\hline S 15 & 31 & 3 & 274.068 & 0.08767 & 298.595 & 909.924 & -0.000027902 & 0.0370835 & 0.689693 \\
\hline S 15 & 31 & 4 & 137.854 & 0.023326 & 811.743 & 913.326 & $-4.0083661676831204^{\prime} 10^{-6}$ & 0.0151454 & -0.498679 \\
\hline S 15 & 31 & 5 & 89.8075 & 0.0629081 & 423.419 & 1498.16 & -0.0000322542 & 0.00880054 & -1.88752 \\
\hline S 15 & 31 & 6 & 375.513 & 0.124366 & 88.858 & 1000. & $-1 \cdot 10-6$ & 0.0265717 & -1.229 \\
\hline S 15 & 31 & 7 & 176.666 & 0.0446057 & 127.522 & 643.676 & -0.0000784173 & 0.0108641 & -1.6212 \\
\hline S 15 & 31 & 8 & 128.27 & 0.0739129 & 239.131 & 1100. & $-1 \cdot 10^{\prime}-6$ & 0.00672417 & 1.5589 \\
\hline S 15 & 31 & 9 & 64.1119 & 0.0170971 & 431.049 & 1482.8 & $-1.0138391028926606^{\prime} 10^{-6}$ & 0.00307385 & 2.6304 \\
\hline S 15 & 31 & 10 & 97.513 & 0.234376 & 439.348 & 1308.64 & $-4.63013^{\prime} 10^{-6}$ & 0.00431328 & 1.05028 \\
\hline S 15 & 31 & 11 & 66.5247 & 0.0977548 & 159.913 & 595.89 & $4.99268^{\prime} 10^{-6}$ & 0.00269631 & -0.190883 \\
\hline 15 & 31 & 12 & 72.7133 & 0.0331407 & 1567.08 & 1336.18 & $-3.34598^{\prime} 10^{-6}$ & 0.00276428 & 3.04985 \\
\hline
\end{tabular}

\section{Sample 15 Scan 32:}

\begin{tabular}{|c|c|c|c|c|c|c|c|c|c|c|}
\hline s & & $\mathrm{T}$ & M & LL & w & $\mathrm{s}$ & $\mathrm{t}$ & a & $\div$ erg & $f$ \\
\hline $\mathrm{s}$ & 15 & 32 & 1 & 1149.73 & 0.0234951 & 246.973 & 1098.76 & 0.0000553471 & 0.683277 & -0.101868 \\
\hline $\mathrm{s}$ & 15 & 32 & 2 & 209.791 & 0.0059998 & 536.657 & 1527.62 & $-4.81428^{\prime} 10^{-6}$ & 0.0597322 & 0.67339 \\
\hline & 15 & 32 & 3 & 368.287 & 0.124691 & 66.0736 & 1000.43 & $-9.94016^{\prime} 10^{-7}$ & 0.0766996 & -1.73487 \\
\hline$S$ & 15 & 32 & 4 & 168.26 & 0.0746337 & 570.016 & 686.108 & -0.0000258749 & 0.0277058 & -0.524096 \\
\hline $\mathrm{s}$ & 15 & 32 & 5 & 87.9362 & 0.0234343 & 823.252 & 936.276 & $-4.37707^{\prime} 10^{-6}$ & 0.0127277 & -1.10595 \\
\hline $\mathrm{s}$ & 15 & 32 & 6 & 102.434 & 0.234513 & 424.468 & 1316.41 & $-5.17688^{\prime} 10^{-6}$ & 0.0134811 & -1.06102 \\
\hline s & 15 & 32 & 7 & 81.9296 & 0.0711768 & 1041.03 & 1362.42 & $-5.33866^{\prime} 10^{-6}$ & 0.00983145 & 1.34584 \\
\hline $\mathrm{s}$ & 15 & 32 & 8 & 192.748 & 0.208936 & 209.59 & 837.676 & -0.0000137261 & 0.0117936 & -0.482402 \\
\hline $\mathrm{s}$ & 15 & 32 & 9 & 113.756 & 0.054329 & 1281.03 & 679.384 & $-6.2116^{\prime} 10^{-6}$ & 0.0111133 & 1.82111 \\
\hline$S$ & 15 & 32 & 10 & 128.601 & 0.091406 & 506.246 & 963.179 & 0.0000148847 & 0.011131 & 1.10053 \\
\hline $\mathrm{s}$ & 15 & 32 & 11 & 82.5668 & 0.0360366 & 414.282 & 591.234 & -0.0000229484 & 0.00642788 & -0.0525994 \\
\hline & 15 & 32 & 12 & 122.743 & 0.136397 & 177.188 & 700.219 & $-9.63674^{\prime} 10^{-7}$ & 0.00485942 & 2.64757 \\
\hline
\end{tabular}




\section{Sample 15 Scan 33:}

\begin{tabular}{|c|c|c|c|c|c|c|c|c|c|c|}
\hline $\mathrm{s}$ & & $\mathrm{T}$ & M & LL & w & $\mathrm{s}$ & $\mathrm{t}$ & a & 응 erg & $f$ \\
\hline & 15 & 33 & 1 & 1155.36 & 0.0233768 & 247.185 & 1099.43 & 0.0000553735 & 0.685056 & -0.121887 \\
\hline $\mathrm{s}$ & 15 & 33 & 2 & 212.598 & 0.00602958 & 537.073 & 1529.77 & $-4.7705072686118415^{\prime} 10^{-6}$ & 0.0601126 & 0.65497 \\
\hline $\mathrm{s}$ & 15 & 33 & 3 & 234.412 & 0.089523 & 195.801 & 999.926 & $-1 \cdot 10^{-6}$ & 0.0495964 & -1.8346 \\
\hline $\mathrm{s}$ & 15 & 33 & 4 & 215.557 & 0.124355 & 257.319 & 1000 . & $-9.99494 \cdot 10^{-7}$ & 0.0362097 & -1.6352 \\
\hline $\mathrm{s}$ & 15 & 33 & 5 & 94.9554 & 0.071812 & 747.762 & 1063.34 & $1.0435^{\prime} 10^{-6}$ & 0.015188 & -3.03547 \\
\hline $\mathrm{s}$ & 15 & 33 & 6 & 166.282 & 0.139839 & 239.187 & 900 . & $-1.0217867446693128^{\prime} 10^{-6}$ & 0.0227549 & -0.232556 \\
\hline $\mathrm{s}$ & 15 & 33 & 7 & 83.3049 & 0.0586791 & 781.322 & 898.199 & $-1.7902900062881998^{\prime} 10^{-6}$ & 0.0103685 & 1.73128 \\
\hline $\mathrm{s}$ & 15 & 33 & 8 & 111.22 & 0.0244099 & 815.469 & 1066.44 & $-4.3821836003208535^{\prime} 10^{-6}$ & 0.0125627 & 2.05324 \\
\hline $\mathrm{s}$ & 15 & 33 & 9 & 110.328 & 0.23422 & 418.627 & 1296.59 & $-3.26763^{\prime} 10^{-6}$ & 0.0111549 & -2.6364 \\
\hline $\mathrm{s}$ & 15 & 33 & 10 & 222.226 & 0.209722 & 225.063 & 862.505 & -0.0000115335 & 0.0116365 & 0.645944 \\
\hline $\mathrm{s}$ & 15 & 33 & 11 & 88.1525 & 0.0349951 & 134.543 & 598.017 & -0.0000478941 & 0.0070874 & -0.105817 \\
\hline & 15 & 33 & 12 & 32.9696 & 0.0792617 & 2568.12 & 2596.26 & $-2.96301 \cdot 10^{-7}$ & 0.00249423 & 0.400342 \\
\hline
\end{tabular}

\section{Sample 15 Scan 34:}

\begin{tabular}{llllll}
\multicolumn{2}{l}{$S$} & $T$ & $M$ & \multicolumn{1}{c}{$L L$} & \multicolumn{1}{c}{ W } \\
$S$ & 15 & 34 & 1 & 1163.2 & 0.0233064 \\
$S$ & 15 & 34 & 2 & 214.95 & 0.00605798 \\
$S$ & 15 & 34 & 3 & 242.407 & 0.0897258 \\
$S$ & 15 & 34 & 4 & 86.8172 & 0.0719902 \\
$S$ & 15 & 34 & 5 & 359.234 & 0.12342 \\
$S$ & 15 & 34 & 6 & 85.0402 & 0.058755 \\
$S$ & 15 & 34 & 7 & 117.627 & 0.0232794 \\
$S$ & 15 & 34 & 8 & 180.677 & 0.207098 \\
$S$ & 15 & 34 & 9 & 99.6198 & 0.235405 \\
$S$ & 15 & 34 & 10 & 149.614 & 0.140935 \\
$S$ & 15 & 34 & 11 & 39.3379 & 0.078458 \\
$S$ & 15 & 34 & 12 & 110.578 & 0.0387454
\end{tabular}

$\begin{array}{llllll}\mathrm{S} & 15 & 34 & 12 & 110.578 & 0.0387454\end{array}$

\begin{tabular}{lcl}
\multicolumn{1}{c}{$\mathrm{s}$} & $\mathrm{t}$ & \multicolumn{1}{c}{$\mathrm{a}$} \\
247.898 & 1099.38 & 0.000055442 \\
539.939 & 1532.99 & $-4.71979^{\prime} 10^{-6}$ \\
169.94 & 1000.12 & $-1 .{ }^{\prime} 10^{-6}$ \\
684.346 & 1036.36 & $1.4135909285149022^{\prime} 10^{-6}$ \\
104.774 & 1000.37 & $-9.75755^{\prime} 10^{-7}$ \\
789.76 & 903.424 & $-1.66791^{\prime} 10^{-6}$ \\
785.875 & 898.857 & $-3.91355^{\prime} 10^{-6}$ \\
234.133 & 879.613 & -0.0000453452 \\
116.515 & 1400.45 & $-1.0021602576455904^{\prime} 10^{-6}$ \\
276.56 & 778.967 & -0.0000412281 \\
2830.24 & 3358.57 & $2.56681^{\prime} 10^{-8}$ \\
353.527 & 610.355 & -0.0000268288
\end{tabular}

\begin{tabular}{ll}
\multicolumn{1}{c}{$\frac{c}{\mathrm{o}} \mathrm{erg}$} & $\mathrm{f}$ \\
0.687516 & -0.134068 \\
0.0602354 & 0.629812 \\
0.0494269 & -1.88238 \\
0.0167537 & -1.18752 \\
0.0554941 & -1.63839 \\
0.0105352 & 1.35748 \\
0.0131716 & -0.321059 \\
0.0167792 & -2.63514 \\
0.00707792 & -2.02034 \\
0.0113472 & -1.49605 \\
0.00271219 & -2.45388 \\
0.00707651 & -0.742452
\end{tabular}

\section{Sample 15 Scan 35:}

\begin{tabular}{llllll} 
S & T & M & \multicolumn{1}{c}{ LL } & \multicolumn{1}{c}{ W } \\
$S$ & 15 & 35 & 1 & 1158.22 & 0.023339 \\
$S$ & 15 & 35 & 2 & 212.969 & 0.00604596 \\
$S$ & 15 & 35 & 3 & 239.31 & 0.089645 \\
$S$ & 15 & 35 & 4 & 90.1614 & 0.0720386 \\
$S$ & 15 & 35 & 5 & 371.613 & 0.1248 \\
$S$ & 15 & 35 & 6 & 87.0168 & 0.0587348 \\
$S$ & 15 & 35 & 7 & 117.541 & 0.0232042 \\
$S$ & 15 & 35 & 8 & 237.418 & 0.210617 \\
$S$ & 15 & 35 & 9 & 100.594 & 0.235472 \\
$S$ & 15 & 35 & 10 & 115.385 & 0.139917 \\
$S$ & 15 & 35 & 11 & 99.6729 & 0.0391224 \\
$S$ & 15 & 35 & 12 & 144.555 & 0.331604
\end{tabular}

\begin{tabular}{|c|c|c|c|c|}
\hline s & $\mathrm{t}$ & a & $\frac{\circ}{6}$ erg & $f$ \\
\hline 48.854 & 1099.32 & 0.0000550565 & 0.685955 & -0.117073 \\
\hline 1.483 & 1536.18 & $-4.64375^{\prime} 10^{-6}$ & 0.0600272 & 0.622393 \\
\hline 0.896 & 1000. & $-9.99519^{\prime} 10^{-7}$ & 0.0497534 & -1.85951 \\
\hline 5.07 & 1030.15 & $1.5292194247746144^{\prime} 10^{-6}$ & 0.0174892 & -0.771723 \\
\hline .6966 & 1000.31 & 0.0000232822 & 0.0565872 & -1.70056 \\
\hline .825 & 895.72 & $-1.7100326348066428^{\prime} 10^{-6}$ & 0.010732 & 1.81246 \\
\hline .539 & 891.71 & $-3.9090450438734335^{\prime} 10^{-6}$ & 0.0130907 & -0.149338 \\
\hline 2.503 & 896.916 & -0.00001152 & 0.0139793 & -0.347192 \\
\hline .405 & 1303.27 & $-5.90034^{\prime} 10^{-6}$ & 0.00870573 & 2.07967 \\
\hline .045 & 762.196 & -0.0000449453 & 0.00896479 & 0.91259 \\
\hline .177 & 622.041 & -0.0000294679 & 0.0069536 & -1.0891 \\
\hline & & 325287 & 00 & \\
\hline
\end{tabular}

\section{Sample 15 Scan 36:}

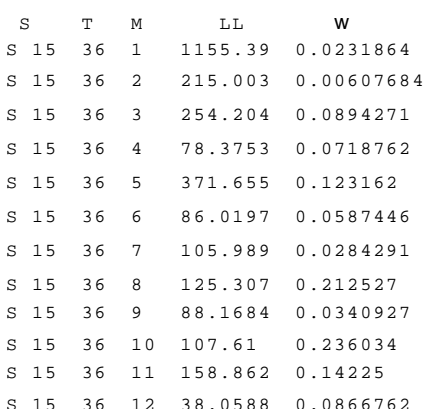

S $15 \quad 36 \quad 12 \quad 38.0588 \quad 0.0866762$

\begin{tabular}{|c|c|c|c|c|}
\hline $\mathrm{s}$ & $t$ & a & $\frac{\circ}{0}$ erg & $f$ \\
\hline 249.527 & 1100.31 & 0.0000551636 & 0.685067 & -0.14607 \\
\hline 542.634 & 1537.83 & $-4.6559605021627125^{\prime} 10^{-6}$ & 0.0607157 & 0.606667 \\
\hline 186.511 & 1000 . & $-1.000011^{\prime} 10^{-6}$ & 0.0517641 & -1.88875 \\
\hline 711.853 & 1050.99 & $1.3350505353707346^{\prime} 10^{-6}$ & 0.0151564 & -2.23472 \\
\hline 95.7148 & 1001.33 & $-1 \cdot 10^{-6}$ & 0.0558405 & -1.75487 \\
\hline 788.665 & 900.518 & $-1.5406324413375074^{\prime} 10^{-6}$ & 0.0107202 & 1.50104 \\
\hline 1542.66 & 1403.36 & $-5.30954^{\prime} 10^{-6}$ & 0.0120016 & -0.508464 \\
\hline 420.748 & 1007.02 & $-5.99082^{\prime} 10^{-6}$ & 0.0126419 & 1.37024 \\
\hline 107.068 & 593.469 & -0.0000489816 & 0.00799188 & 0.0251134 \\
\hline 4 & 1311.55 & $-9.32997^{\prime} 10^{-6}$ & 0.00884577 & 0.191235 \\
\hline 94.815 & 792.344 & -0.0000409292 & 0.0114364 & 2.86958 \\
\hline 63.5 & 373.96 & $-5.57992^{\prime} 10^{-7}$ & 0.00248045 & 1.27401 \\
\hline
\end{tabular}




\section{Sample 15 Scan 37:}

\begin{tabular}{|c|c|c|c|c|c|c|c|c|c|c|}
\hline $\mathrm{S}$ & & $\mathrm{T}$ & M & LL & w & $\mathrm{s}$ & $\mathrm{t}$ & a & $\div$ erg & $f$ \\
\hline $\mathrm{s}$ & 15 & 37 & 1 & 1153.1 & 0.0231082 & 250.067 & 1100.96 & 0.0000549779 & 0.684344 & -0.154391 \\
\hline & 15 & 37 & 2 & 216.169 & 0.0060861 & 543.981 & 1539.91 & $-4.65352^{\prime} 10^{-6}$ & 0.0611477 & 0.599211 \\
\hline s & 15 & 37 & 3 & 259.582 & 0.0893136 & 195.488 & 1000 . & $-9.99722^{\prime} 10^{-7}$ & 0.0524707 & -1.89758 \\
\hline S & 15 & 37 & 4 & 73.8035 & 0.071815 & 724.344 & 1055.75 & $1.29836^{\prime} 10^{-6}$ & 0.0142732 & -2.58358 \\
\hline $\mathrm{s}$ & 15 & 37 & 5 & 358.112 & 0.12334 & 95.037 & 1000.82 & $-1 \cdot 10^{-6}$ & 0.0551667 & -1.70084 \\
\hline $\mathrm{s}$ & 15 & 37 & 6 & 85.6902 & 0.0587302 & 788.873 & 900.415 & $-1.55624^{\prime} 10^{-6}$ & 0.0107726 & 1.50487 \\
\hline $\mathrm{s}$ & 15 & 37 & 7 & 105.202 & 0.0284432 & 1509.73 & 1400.34 & $-5.37995^{\prime} 10^{-6}$ & 0.0120234 & -0.414003 \\
\hline $\mathrm{s}$ & 15 & 37 & 8 & 243.397 & 0.209641 & 202.439 & 881.16 & -0.0000133545 & 0.0136172 & 3.06187 \\
\hline & 15 & 37 & 9 & 87.2859 & 0.0340002 & 109.587 & 592.907 & -0.000049967 & 0.00792095 & 0.0429253 \\
\hline & 15 & 37 & 10 & 102.936 & 0.237336 & 252.148 & 1354.7 & $-6.58388^{\prime} 10^{-6}$ & 0.00849462 & 2.5518 \\
\hline & 15 & 37 & 11 & 8 & & 07 & .32 & -0.0000128378 & 0.00464829 & 2.0938 \\
\hline & 15 & 37 & 12 & 49.1515 & 0.0179587 & 1496.13 & 1546.76 & -0.0000129096 & 0.0035349 & 2.05369 \\
\hline
\end{tabular}

\section{Sample 15 Scan 38:}

\begin{tabular}{llllll}
$S$ & $T$ & $M$ & \multicolumn{1}{c}{$L I$} & \multicolumn{1}{c}{$W$} \\
$S$ & 15 & 38 & 1 & 1160.91 & 0.0230193 \\
$S$ & 15 & 38 & 2 & 221.127 & 0.00609733 \\
$S$ & 15 & 38 & 3 & 265.915 & 0.0892983 \\
$S$ & 15 & 38 & 4 & 72.953 & 0.071766 \\
$S$ & 15 & 38 & 5 & 360.662 & 0.123066 \\
$S$ & 15 & 38 & 6 & 88.9574 & 0.0587312 \\
$S$ & 15 & 38 & 7 & 110.6 & 0.0283359 \\
$S$ & 15 & 38 & 8 & 131.803 & 0.212479 \\
$S$ & 15 & 38 & 9 & 92.9055 & 0.0341352 \\
$S$ & 15 & 38 & 10 & 111.785 & 0.236542 \\
$S$ & 15 & 38 & 11 & 150.765 & 0.140627 \\
$S$ & 15 & 38 & 12 & 58.0778 & 0.0179603
\end{tabular}

\begin{tabular}{|c|c|c|}
\hline $\mathrm{s}$ & $\mathrm{t}$ & a \\
\hline 0.782 & 1101.57 & 0.0000550712 \\
\hline 4.391 & 1539.84 & $-4.68861 \cdot 10^{-6}$ \\
\hline 3.222 & 1000 & $-1.0001544443573796^{\prime} 10^{-6}$ \\
\hline 1.243 & 1064.51 & $1.1884448093533903^{\prime} 10^{-6}$ \\
\hline 939 & 999.755 & $-1 \cdot 10^{\prime}-6$ \\
\hline .114 & 899.477 & $-1.4557274113820272^{\prime} 10^{-6}$ \\
\hline 8.82 & 1395.16 & $-5.30535^{\prime} 10^{-6}$ \\
\hline 3.15 & 1028.31 & $-5.81457^{\prime} 10^{-6}$ \\
\hline 6.879 & 593.56 & -0.0000512317 \\
\hline .123 & 1309.42 & -0.0000137025 \\
\hline 85 & 768.896 & -0.0000440703 \\
\hline & 1545.02 & -0.0000129913 \\
\hline
\end{tabular}

\begin{tabular}{ll}
\multicolumn{1}{c}{ o $\mathrm{erg}$} & \multicolumn{1}{c}{$f$} \\
0.6868 & -0.170611 \\
0.0619167 & 0.597999 \\
0.0531324 & -1.89174 \\
0.0138424 & 3.10028 \\
0.0553132 & -1.53565 \\
0.0108602 & 1.55548 \\
0.0122224 & -0.269659 \\
0.0129056 & -3.07416 \\
0.00812721 & 0.0313512 \\
0.00882871 & 0.889877 \\
0.0104469 & -0.0302609 \\
0.0036224 & 2.09402
\end{tabular}

Sample 15 Scan 39:

\begin{tabular}{llllll}
$S$ & $T$ & $M$ & LL & \multicolumn{1}{c}{$W$} \\
$S$ & 15 & 39 & 1 & 1295.01 & 0.0227607 \\
$S$ & 15 & 39 & 2 & 279.573 & 0.00610588 \\
$S$ & 15 & 39 & 3 & 285.329 & 0.0890612 \\
$S$ & 15 & 39 & 4 & 91.1013 & 0.0717739 \\
$S$ & 15 & 39 & 5 & 96.4657 & 0.0281887 \\
$S$ & 15 & 39 & 6 & 437.686 & 0.122498 \\
$S$ & 15 & 39 & 7 & 133.918 & 0.0585969 \\
$S$ & 15 & 39 & 8 & 117.967 & 0.0337112 \\
$S$ & 15 & 39 & 9 & 130.376 & 0.212552 \\
$S$ & 15 & 39 & 10 & 70.9779 & 0.0176589 \\
$S$ & 15 & 39 & 11 & 168.205 & 0.139691 \\
$S$ & 15 & 39 & 12 & 46.2157 & 0.0802062
\end{tabular}

\begin{tabular}{|c|c|c|}
\hline s & $\mathrm{t}$ & a \\
\hline 51.179 & 1105.7 & 0.0000549395 \\
\hline 6.588 & 1543.68 & $-4.68721 \cdot 10^{-6}$ \\
\hline 7.538 & 1000.12 & $-1.0002883833439113^{\prime} 10^{-6}$ \\
\hline 4.499 & 1067.21 & $1.2287833201922289^{\prime} 10^{-6}$ \\
\hline 94.91 & 1394.34 & $-5.13289^{\prime} 10^{-6}$ \\
\hline 3.063 & 1000 & $-9.37278^{\prime} 10^{-7}$ \\
\hline 9.515 & 871.928 & $-1.7782663022174238^{\prime} 10^{-6}$ \\
\hline 7.205 & 594.707 & -0.0000505989 \\
\hline 3.833 & 1035.3 & $-5.73972^{\prime} 10^{-6}$ \\
\hline 03.23 & 1537.48 & -0.0000130104 \\
\hline 5.02 & 759.188 & -0.0000475437 \\
\hline 5.2 & 2517.82 & $-6.14408^{\prime} 10^{-7}$ \\
\hline
\end{tabular}

\begin{tabular}{ll}
\multicolumn{1}{c}{$\quad$ org } & \multicolumn{1}{c}{$f$} \\
0.726105 & -0.214542 \\
0.0665106 & 0.590884 \\
0.0495182 & -1.7512 \\
0.013613 & 3.02716 \\
0.0131247 & -0.199551 \\
0.0441941 & -1.37069 \\
0.0107043 & -2.9682 \\
0.00831211 & 0.0531399 \\
0.00811453 & 1.90128 \\
0.00399255 & 2.29664 \\
0.0083879 & 1.60311 \\
0.00206809 & -0.231004
\end{tabular}

\section{Sample 15 Scan 40:}

\begin{tabular}{llllll} 
S & T & M & LL & \multicolumn{1}{c}{ W } \\
$S$ & 15 & 40 & 1 & 1543.48 & 0.0218286 \\
$S$ & 15 & 40 & 2 & 445.956 & 0.00610146 \\
$S$ & 15 & 40 & 3 & 133.728 & 0.0234266 \\
$S$ & 15 & 40 & 4 & 285.155 & 0.0885666 \\
$S$ & 15 & 40 & 5 & 101.425 & 0.0715205 \\
$S$ & 15 & 40 & 6 & 112.163 & 0.0587505 \\
$S$ & 15 & 40 & 7 & 424.457 & 0.121119 \\
$S$ & 15 & 40 & 8 & 173.454 & 0.0389506 \\
$S$ & 15 & 40 & 9 & 93.872 & 0.0328436 \\
$S$ & 15 & 40 & 10 & 93.0342 & 0.0180972 \\
$S$ & 15 & 40 & 11 & 127.196 & 0.211871 \\
$S$ & 15 & 40 & 12 & 53.1877 & 0.0991259
\end{tabular}

\begin{tabular}{|c|c|c|c|c|}
\hline $\mathrm{s}$ & $\mathrm{t}$ & a & $\frac{\circ}{6}$ erg & $f$ \\
\hline 49.928 & 1117.68 & 0.000054783 & 0.786364 & -0.379056 \\
\hline 0.838 & 1547.35 & $-4.685911^{\prime} 10^{-6}$ & 0.0763877 & 0.594296 \\
\hline 8.438 & 918.11 & $-3.85282^{\prime} 10^{-6}$ & 0.0169673 & -0.6282 \\
\hline 5.747 & 1000 . & $-1 \cdot 10^{-6}$ & 0.0280627 & -1.50071 \\
\hline 2.944 & 1098.07 & $5.82499^{\prime} 10^{-7}$ & 0.00873022 & 1.1967 \\
\hline 027 & 900.867 & $-1.4612656688385938^{\prime} 10^{-} 6$ & 0.00867747 & 1.83783 \\
\hline 5569 & 1000 . & $-1.0692734299842495^{\prime} 10^{-6}$ & 0.022623 & -1.22056 \\
\hline 3.433 & 619.995 & -0.000026252 & 0.00803931 & -0.900285 \\
\hline 3.012 & 1278.72 & $-3.4934496041316275^{\prime} 10^{-6}$ & 0.00380307 & -1.40551 \\
\hline .18 & 1658.2 & $-4.73863^{\prime} 10^{-7}$ & 0.0034328 & -0.434819 \\
\hline & 1002.62 & $-6.9318054202749325^{\prime} 10^{-6}$ & 0.00420508 & 2.59801 \\
\hline 22 & 1354.03 & -0.0000131856 & 0.00160584 & 0.805085 \\
\hline
\end{tabular}




\section{Sample 15 Scan 41:}

\begin{tabular}{|c|c|c|c|c|c|c|c|c|c|c|}
\hline $\mathrm{s}$ & & $\mathrm{T}$ & M & LL & w & s & $\mathrm{t}$ & a & 음 erg & $f$ \\
\hline & 15 & 41 & 1 & 1535.09 & 0.0218692 & 250.592 & 1116.93 & 0.0000549792 & 0.784564 & -0.375226 \\
\hline & 15 & 41 & 2 & 441.587 & 0.00611178 & 547.772 & 1544.56 & $-4.77389^{\prime} 10^{-6}$ & 0.076445 & 0.605975 \\
\hline & 15 & 41 & 3 & 133.379 & 0.0234723 & 797.471 & 921.466 & $-3.8750273728399305^{\prime} 10^{-6}$ & 0.0171479 & -0.706468 \\
\hline & 15 & 41 & 4 & 314.262 & 0.0885413 & 193.248 & 1000 . & $-1.0146970080868854^{\prime} 10^{-6}$ & 0.032051 & -1.46508 \\
\hline & 15 & 41 & 5 & 130.779 & 0.0717487 & 725.732 & 1070.24 & $1.3968614421095063^{\prime} 10^{-6}$ & 0.010803 & 3.06321 \\
\hline & 15 & 41 & 6 & 184.285 & 0.0559427 & -84.6369 & 602.096 & 0.000414561 & 0.0130132 & -0.376474 \\
\hline$S$ & 15 & 41 & 7 & 90.7772 & 0.0589162 & 1098.33 & 1284.51 & $7.66796^{\prime} 10^{-6}$ & 0.00558011 & -2.71361 \\
\hline & 15 & 41 & 8 & 535.692 & 0.118373 & 93.1474 & 999.989 & $-9.46429^{\prime} 10^{-7}$ & 0.0206948 & -1.09376 \\
\hline & 15 & 41 & 9 & 108.753 & 0.0355397 & 756.6 & 1185.09 & $2.57294^{\prime} 10^{-6}$ & 0.00391867 & 1.58534 \\
\hline$S$ & 15 & 41 & 10 & 138.808 & 0.210126 & 236.789 & 906.222 & -0.0000108207 & 0.00382294 & -1.98283 \\
\hline & 15 & 41 & 11 & 68.4399 & 0.0270779 & 485.626 & 423.614 & $-5.19018^{\prime} 10^{-6}$ & 0.00200343 & -0.908131 \\
\hline & 15 & 41 & 12 & 2.532 & .0187136 & 658.358 & 1646.86 & $-5.47371 \cdot 10^{-6}$ & 0.00351057 & -0.01014 \\
\hline
\end{tabular}

\section{Sample 15 Scan 42:}

\begin{tabular}{|c|c|c|c|c|c|c|c|c|c|c|}
\hline & & $\mathrm{T}$ & м & LL & w & $\mathrm{s}$ & $\mathrm{t}$ & a & $\%$ erg & f \\
\hline & 15 & 42 & 1 & 1565.36 & 0.0217434 & 251.008 & 1118.97 & 0.0000547308 & 0.790987 & -0.390447 \\
\hline & 15 & 42 & 2 & 466.417 & 0.00610158 & 549.631 & 1546.99 & $-4.73535 \times 10^{-6}$ & 0.077413 & 0.602938 \\
\hline & 15 & 42 & 3 & 144.07 & 0.0234849 & 796.765 & 924.317 & $-3.87719^{\prime} 10^{-6}$ & 0.0174265 & -0.745866 \\
\hline & 15 & 42 & 4 & 339.213 & 0.0884209 & 212.707 & 1000 . & $-9.37278^{\prime} 10^{-7}$ & 0.0314538 & -1.40035 \\
\hline & 15 & 42 & 5 & 124.049 & 0.0716288 & 751.94 & 1084.54 & $1.2747326976505238^{\prime} 10^{-6}$ & 0.009449 & 2.1047 \\
\hline & 15 & 42 & 6 & 200.956 & 0.0559845 & -85.423 & 602.456 & 0.000411541 & 0.0130248 & -0.351158 \\
\hline & 15 & 42 & 7 & 98.7213 & 0.0584486 & 1074.81 & 1308.36 & $7.72065^{\prime} 10^{-6}$ & 0.00550062 & 2.20302 \\
\hline & 15 & 42 & 8 & 388.602 & 0.118412 & 248.687 & 1000 . & $-9.97082^{\prime} 10^{-7}$ & 0.0127513 & -0.948771 \\
\hline & 15 & 42 & 9 & 280.397 & 0.139257 & 228.779 & 900. & $-1.00582^{\prime} 10^{-6}$ & 0.00518771 & 0.131206 \\
\hline & 15 & 42 & 10 & 118.981 & 0.0354231 & 743.425 & 1203.44 & $2.4345291153236805^{\prime} 10^{-6}$ & 0.0039345 & 0.98551 \\
\hline S & 15 & 42 & 11 & 65.4118 & 0.0270292 & 470.35 & 421.619 & $-5.4982661405546625^{\prime} 10^{-6}$ & 0.00197173 & -0.825873 \\
\hline & 15 & 42 & 12 & 134.827 & 0.018334 & 616.159 & 1609.75 & $-8.74756^{\prime} 10^{-6}$ & 0.00367908 & 0.88227 \\
\hline
\end{tabular}

\section{Sample 15 Scan 43:}

\begin{tabular}{|c|c|c|c|c|c|c|c|c|c|}
\hline $\mathrm{s}$ & $\mathrm{T}$ & M & LL & w & $\mathrm{s}$ & $\mathrm{t}$ & a & $\div$ erg & $\mathrm{f}$ \\
\hline S 15 & 43 & 1 & 1566.43 & 0.0214789 & 251.753 & 1121. & 0.0000547331 & 0.791211 & -0.434551 \\
\hline 15 & 43 & 2 & 477.271 & 0.00612856 & 550.337 & 1549.14 & $-4.73472^{\prime} 10^{-6}$ & 0.0787355 & 0.588161 \\
\hline 15 & 43 & 3 & 149.748 & 0.0235515 & 807.687 & 934.223 & $-3.92764^{\prime} 10^{-6}$ & 0.0178478 & -0.970845 \\
\hline 15 & 43 & 4 & 326.836 & 0.0885311 & 209.062 & 1000 . & $-1 \cdot 10^{-6}$ & 0.0305339 & -1.44123 \\
\hline 15 & 43 & 5 & 118.037 & 0.0716284 & 748.504 & 1081.7 & $1.3428769133572002^{\prime} 10^{-6}$ & 0.00890061 & 2.27953 \\
\hline 15 & 43 & 6 & 196.645 & 0.055987 & -85.6874 & 602.049 & 0.000406245 & 0.0126824 & -0.337481 \\
\hline 15 & 43 & 7 & 95.2115 & 0.0583342 & 1085.98 & 1312.9 & $7.75478^{\prime} 10^{-6}$ & 0.00529979 & 1.93808 \\
\hline 15 & 43 & 8 & 512.464 & 0.118332 & 101.366 & 1000 . & $-9.37278^{\prime} 10^{-7}$ & 0.0174427 & -1.08229 \\
\hline 15 & 43 & 9 & 111.097 & 0.0356572 & 737.609 & 1156.95 & $2.53807^{\prime} 10^{-6}$ & 0.00374462 & 2.6278 \\
\hline 15 & 43 & 10 & 126.872 & 0.0172415 & 445.6 & 1542.97 & $-6.66661 \cdot 10^{-6}$ & 0.00379719 & 1.97515 \\
\hline 15 & 43 & 11 & 223.391 & 0.207865 & 84.675 & 897.947 & -0.000013892 & 0.00434221 & -0.0878981 \\
\hline 15 & 43 & 12 & 88.608 & 0.0270608 & 459.169 & 426.456 & $-5.87138^{\prime} 10^{-6}$ & 0.00201204 & -0.942702 \\
\hline
\end{tabular}

\section{Sample 15 Scan 44:}

\begin{tabular}{|c|c|c|c|c|c|c|c|c|c|c|}
\hline & & $\mathrm{T}$ & $\mathrm{M}$ & LL & w & $\mathrm{s}$ & $\mathrm{t}$ & a & 응 erg & $f$ \\
\hline & 15 & 44 & 1 & 1483.98 & 0.0215713 & 252.917 & 1118.15 & 0.0000546687 & 0.773266 & -0.4143 \\
\hline & 15 & 44 & 2 & 416.25 & 0.0061255 & 549.544 & 1547.23 & $-4.73355^{\prime} 10^{-6}$ & 0.0767582 & 0.589249 \\
\hline & 15 & 44 & 3 & 124.984 & 0.0236131 & 812.794 & 939.113 & $-3.94156^{\prime} 10^{-6}$ & 0.0174241 & -1.12649 \\
\hline & 15 & 44 & 4 & 333.138 & 0.0884013 & 203.561 & 1001.1 & $-9.98683^{\prime} 10^{-7}$ & 0.0366617 & -1.67784 \\
\hline & 15 & 44 & 5 & 107.302 & 0.0716659 & 24.834 & 1078.84 & $1.66386887666666844^{\prime} 10^{-6}$ & 0.00958562 & 2.29488 \\
\hline & 15 & 44 & 6 & 183.953 & 0.0316647 & 88.9677 & 626.955 & 0.000409998 & 0.0142192 & -1.47498 \\
\hline & 15 & 44 & 7 & 448.813 & 0.1194 & 98.2065 & 1000. & $-1.03871^{\prime} 10^{-6}$ & 0.0228113 & -1.22384 \\
\hline & 15 & 44 & 8 & 18.977 & 0.0579092 & 1125.6 & 1350.9 & $7.80988^{\prime} 10^{-6}$ & 0.00533534 & -0.35042 \\
\hline & 15 & 44 & 9 & 233.722 & 0.209821 & 186.757 & 864.415 & -0.0000108055 & 0.00528877 & 0.45618 \\
\hline & 15 & 44 & 10 & 124.89 & 0.0334563 & 1813.53 & 1356.11 & $-3.5239354951876224^{\prime} 10^{-6}$ & 0.00433696 & 2.38329 \\
\hline & 15 & 44 & 11 & 115.94 & 0.0168066 & 394.091 & 1501.93 & $-7.73927^{\prime} 10^{-6}$ & 0.003569 & 2.66626 \\
\hline & 15 & 44 & 12 & 49.3241 & 0.0385251 & 1297.84 & 1136.02 & $-1.6917662679016509^{\prime} 10^{-6}$ & 0.00139727 & -2.9795 \\
\hline
\end{tabular}




\section{Sample 15 Scan 45:}

\begin{tabular}{|c|c|c|c|c|c|c|c|c|c|c|}
\hline s & & $\mathrm{T}$ & M & LL & w & $\mathrm{s}$ & $\mathrm{t}$ & a & $\therefore$ erg & f \\
\hline $\mathrm{s}$ & 15 & 45 & 1 & 1435.44 & 0.0215073 & 253.918 & 1117.27 & 0.0000547958 & 0.761989 & -0.425479 \\
\hline & 15 & 45 & 2 & 387.564 & 0.0061617 & 551.765 & 1548.55 & $-4.74471 \cdot 10^{-6}$ & 0.0760711 & 0.569432 \\
\hline & 15 & 45 & 3 & 116.936 & 0.0236237 & 822.344 & 936.356 & $-3.9103783230097994^{\prime} 10^{-6}$ & 0.0176951 & -1.09117 \\
\hline & 15 & 45 & 4 & 334.504 & 0.0885075 & 200.243 & 1000.2 & $-1 \cdot 10^{-6}$ & 0.0400998 & -1.67763 \\
\hline & 15 & 45 & 5 & 100.688 & 0.071648 & 719.29 & 1084.12 & $1.8134899184879415^{\prime} 10^{-6}$ & 0.00982175 & 1.82591 \\
\hline & 15 & 45 & 6 & 227.766 & 0.0569013 & 147.641 & 600. & $-9.76042^{\prime} 10^{-7}$ & 0.00974762 & 0.227433 \\
\hline & 15 & 45 & 7 & 242.737 & 0.12149 & 255.227 & 1000 . & $-1.0387642505881289^{\prime} 10^{-6}$ & 0.0165143 & -1.32782 \\
\hline & 15 & 45 & 8 & 96.4718 & 0.139211 & 323.745 & 899.919 & $4.26524^{\prime} 10^{-6}$ & 0.00611157 & -0.245914 \\
\hline $\mathrm{s}$ & 15 & 45 & 9 & 69.0983 & 0.0336863 & 911.134 & 1135.65 & -0.0000134907 & 0.00402889 & -2.47655 \\
\hline & 15 & 45 & 10 & 88.3392 & 0.0124326 & 4042.15 & 2482.92 & $2.61802^{\prime} 10^{-6}$ & 0.00476135 & -0.0672575 \\
\hline & 15 & 45 & 11 & 211.831 & 0.210513 & 223.115 & 900.053 & -0.0000107099 & 0.00652291 & -0.776545 \\
\hline & 15 & 45 & 12 & 105.626 & 0.0589429 & 336.563 & 1319. & $2.3945014794242255^{\prime} 10^{-6}$ & 0.00451374 & 1.75692 \\
\hline
\end{tabular}

Sample 15 Scan 46:

\begin{tabular}{|c|c|c|c|c|c|c|c|c|c|c|}
\hline $\mathrm{s}$ & & $\mathrm{T}$ & M & LL & w & $\mathrm{s}$ & $\mathrm{t}$ & a & $\frac{\circ}{\partial}$ erg & $f$ \\
\hline & 15 & 46 & 1 & 1467.66 & 0.0211967 & 254.006 & 1120.91 & 0.0000546492 & 0.769535 & -0.475541 \\
\hline $\mathrm{S}$ & 15 & 46 & 2 & 418.205 & 0.00615535 & 551.784 & 1549.15 & $-4.7606390988748164^{\prime} 10^{-6}$ & 0.0783291 & 0.576675 \\
\hline $\mathrm{s}$ & 15 & 46 & 3 & 128.02 & 0.0236953 & 820.265 & 946.051 & $-3.90944^{\prime} 10^{-6}$ & 0.0180833 & -1.29019 \\
\hline $\mathrm{S}$ & 15 & 46 & 4 & 336.591 & 0.0883064 & 202.922 & 1000.54 & $-9.90677^{\prime} 10^{-7}$ & 0.0376142 & -1.64839 \\
\hline $\mathrm{s}$ & 15 & 46 & 5 & 73.3236 & 0.0654573 & 259.598 & 1509.57 & -0.0000261106 & 0.0067012 & -2.78454 \\
\hline $\mathrm{s}$ & 15 & 46 & 6 & 307.457 & 0.12128 & 112.401 & 999.989 & $-9.99908^{\prime} 10^{-7}$ & 0.0212152 & -1.25002 \\
\hline S & 15 & 46 & 7 & 105.812 & 0.0726039 & 451.812 & 830.026 & $-1.6768178350429833^{\prime} 10^{-6}$ & 0.00671386 & 1.56283 \\
\hline $\mathrm{s}$ & 15 & 46 & 8 & 208.452 & 0.0473767 & 68.0568 & 613.472 & 0.000073332 & 0.0113184 & -0.666202 \\
\hline $\mathrm{s}$ & 15 & 46 & 9 & 86.7296 & 0.0357645 & 882.543 & 1106.91 & $2.3384218110662045^{\prime} 10^{-6}$ & 0.00405685 & -2.02321 \\
\hline S & 15 & 46 & 10 & 185.737 & 0.209477 & 231.01 & 886.295 & -0.0000153005 & 0.00587995 & 2.14044 \\
\hline $\mathrm{s}$ & 15 & 46 & 11 & 248.806 & 0.192333 & 233.267 & 914.107 & $-8.70463^{\prime} 10^{-6}$ & 0.00457371 & 3.0541 \\
\hline & 15 & 46 & 12 & 121.562 & 0.0177634 & 476.313 & 1564.01 & $-8.03897^{\prime} 10^{-6}$ & 0.00392869 & 1.64488 \\
\hline
\end{tabular}

Sample 15 Scan 47:

\begin{tabular}{|c|c|c|c|c|c|c|c|c|c|}
\hline S & $\mathrm{T}$ & M & LL & w & $\mathrm{s}$ & $\mathrm{t}$ & a & $\frac{\circ}{6}$ erg & $f$ \\
\hline 15 & 47 & 1 & 1505.1 & 0.0209658 & 254.888 & 1123.99 & 0.000054552 & 0.778004 & -0.513053 \\
\hline 15 & 47 & 2 & 457.201 & 0.00620696 & 550.847 & 1553.7 & $-4.79862^{\prime} 10^{-6}$ & 0.0809705 & 0.556026 \\
\hline 15 & 47 & 3 & 143.575 & 0.0237268 & 839.042 & 958.5 & $-4.13875^{\prime} 10^{-6}$ & 0.0186296 & -1.51926 \\
\hline 15 & 47 & 4 & 352.563 & 0.0882115 & 205.928 & 1000. & $-10^{\prime} 10^{-6}$ & 0.0356988 & -1.50509 \\
\hline 15 & 47 & 5 & 70.6373 & 0.0607242 & 403.582 & 1518.97 & 0.0000229387 & 0.00579399 & 2.56621 \\
\hline 15 & 47 & 6 & 117.314 & 0.0589611 & 523.784 & 775.616 & -0.0000105948 & 0.0087627 & -3.04479 \\
\hline 15 & 47 & 7 & 205.52 & 0.121248 & 258.015 & 1000. & $-9.99986^{\prime} 10^{-7}$ & 0.0122218 & -1.13087 \\
\hline 15 & 47 & 8 & 132.724 & 0.0355636 & 345.108 & 567.305 & -0.0000230223 & 0.00723101 & 0.956059 \\
\hline S 15 & 47 & 9 & 117.874 & 0.0701534 & 318.369 & 636.985 & -0.0000130229 & 0.00566472 & -2.94477 \\
\hline 15 & 47 & 10 & 85.2423 & 0.0183246 & 987.944 & 1671.79 & $-6.45416^{\prime} 10^{-7}$ & 0.00369991 & -0.614166 \\
\hline 15 & 47 & 11 & 111.377 & 0.106786 & 270.373 & 1000 . & $7.19582^{\prime} 10^{-6}$ & 0.00432607 & -1.03274 \\
\hline 15 & 47 & 12 & 105.696 & 0.139331 & 317.997 & 899.953 & $5.74235^{\prime} 10^{-6}$ & 0.00373657 & -0.180746 \\
\hline
\end{tabular}

\section{Sample 15 Scan 48:}

\begin{tabular}{|c|c|c|c|c|c|c|c|c|c|}
\hline $\mathrm{s}$ & $\mathrm{T}$ & M & LL & w & s & $\mathrm{t}$ & a & $\%$ erg & $f$ \\
\hline S 15 & 48 & 1 & 1240.4 & 0.0216674 & 257.554 & 1112.42 & 0.0000548146 & 0.710731 & -0.389889 \\
\hline 15 & 48 & 2 & 280.461 & 0.00618345 & 550.527 & 1546.66 & $-4.79938^{\prime} 10^{-6}$ & 0.0704704 & 0.566909 \\
\hline 15 & 48 & 3 & 348.167 & 0.0884296 & 198.398 & 999.996 & $-1 \cdot 10^{-6}$ & 0.0595108 & -1.84608 \\
\hline 15 & 48 & 4 & 102.712 & 0.0269728 & 1039.78 & 1292.54 & $-4.91142^{\prime} 10^{-6}$ & 0.0154081 & 2.58526 \\
\hline 15 & 48 & 5 & 299.446 & 0.121704 & 110.212 & 1000 . & $-9.99722^{\prime} 10^{-7}$ & 0.0362891 & -1.44508 \\
\hline 15 & 48 & 6 & 112.521 & 0.0715439 & 711.674 & 1103.26 & $2.3415347045509213^{\prime} 10^{-6}$ & 0.0112967 & 0.17683 \\
\hline 15 & 48 & 7 & 127.414 & 0.0561879 & 152.959 & 593.186 & 0.0000212116 & 0.0113412 & 0.292251 \\
\hline S 15 & 48 & 8 & 75.2693 & 0.0276729 & 600.835 & 723.439 & $-5.00106^{\prime} 10^{-6}$ & 0.0060552 & 3.11539 \\
\hline 15 & 48 & 9 & 447.811 & 0.195188 & -114.729 & 900.004 & $-1.0008251420417373^{\prime} 10^{-6}$ & 0.0199461 & -0.638078 \\
\hline 15 & 48 & 10 & 129.5 & 0.236334 & 160.339 & 1373.73 & $-3.7507551067025864^{\prime} 10^{-6}$ & 0.00617145 & -1.94453 \\
\hline 15 & 48 & 11 & 143.82 & 0.129203 & 119.696 & 600. & $-1 \cdot 10^{-6}$ & 0.00476479 & -2.43406 \\
\hline 15 & 48 & 12 & 129.027 & 0.057766 & 725.506 & 1370.3 & $6.85134^{\prime} 10^{-6}$ & 0.00563779 & -1.68217 \\
\hline
\end{tabular}




\section{Sample 15 Scan 49:}

\begin{tabular}{|c|c|c|c|c|c|c|c|c|c|c|}
\hline $\mathrm{s}$ & & $\mathrm{T}$ & M & LL & w & $\mathrm{s}$ & $t$ & a & $\frac{\circ}{0}$ erg & $\mathrm{f}$ \\
\hline$S$ & 15 & 49 & 1 & 1529.56 & 0.0207556 & 255.849 & 1126.05 & 0.0000543233 & 0.78337 & -0.541363 \\
\hline & 15 & 49 & 2 & 484.735 & 0.00618993 & 554.376 & 1554.58 & $-4.76973^{\prime} 10^{-6}$ & 0.0826928 & 0.55801 \\
\hline & 15 & 49 & 3 & 157.206 & 0.0237588 & 829.546 & 956.527 & $-3.95345^{\prime} 10^{-6}$ & 0.0192284 & -1.48522 \\
\hline & 15 & 49 & 4 & 365.51 & 0.0879252 & 206.488 & 1000 & $-9.9875^{\prime} 10^{-7}$ & 0.0342834 & -1.47784 \\
\hline & 15 & 49 & 5 & 116.553 & 0.0588237 & 772.548 & 858.668 & $-7.33128^{\prime} 10^{-7}$ & 0.00865384 & -2.1268 \\
\hline & 15 & 49 & 6 & 118.192 & 0.0714997 & 705.98 & 1103.57 & $2.02121^{\prime} 10^{-6}$ & 0.00780379 & 0.534637 \\
\hline & 15 & 49 & 7 & 208.242 & 0.121464 & 254.792 & 1000. & $-1.0148005173694972^{\prime} 10^{-6}$ & 0.0105768 & -1.14305 \\
\hline 5 & 15 & 49 & 8 & 177.101 & 0.0389107 & 369.277 & 620.239 & -0.0000259776 & 0.00837896 & -0.917366 \\
\hline & 15 & 49 & 9 & 98.8816 & 0.0192831 & 1652.38 & 2218.74 & $-7.63476^{\prime} 10^{-7}$ & 0.00407158 & 1.6605 \\
\hline & 15 & 49 & 10 & 94.6064 & 0.032342 & 546.951 & 1197.6 & $-3.6103287403831063^{\prime} 10^{-6}$ & 0.00353588 & 1.28759 \\
\hline & 15 & 49 & 11 & 114.723 & 0.107402 & 265.332 & 999.987 & $-9.37281 \cdot 10^{-7}$ & 0.00384087 & -0.827584 \\
\hline & 15 & 49 & 12 & 367.969 & 0.194772 & 72.6756 & 899.975 & $-1.0387642505881289^{\prime} 10^{-6}$ & 0.00727743 & -0.56194 \\
\hline
\end{tabular}

\section{Sample 15 Scan 50:}

\begin{tabular}{llllll}
$S$ & $T$ & $M$ & \multicolumn{1}{c}{ LL } & \multicolumn{1}{c}{ W } \\
S & 15 & 50 & 1 & 1592.69 & 0.0203281 \\
$S$ & 15 & 50 & 2 & 561.841 & 0.00620061 \\
$S$ & 15 & 50 & 3 & 190.981 & 0.023745 \\
$S$ & 15 & 50 & 4 & 358.079 & 0.0879229 \\
$S$ & 15 & 50 & 5 & 121.155 & 0.0588001 \\
$S$ & 15 & 50 & 6 & 105.918 & 0.0712959 \\
$S$ & 15 & 50 & 7 & 162.177 & 0.0385914 \\
$S$ & 15 & 50 & 8 & 240.826 & 0.12174 \\
$S$ & 15 & 50 & 9 & 122.97 & 0.0195869 \\
$S$ & 15 & 50 & 10 & 114.322 & 0.0323314 \\
$S$ & 15 & 50 & 11 & 151.366 & 0.106845 \\
$S$ & 15 & 50 & 12 & 410.113 & 0.194454
\end{tabular}

\section{Sample 15 Scan 51:}

\begin{tabular}{lllll}
\multicolumn{1}{c}{$\mathrm{s}$} & \multicolumn{1}{c}{$\mathrm{t}$} & \multicolumn{1}{c}{$\mathrm{a}$} & \multicolumn{1}{c}{$\mathrm{o}$ erg } & $f$ \\
255.224 & 1131.38 & 0.0000541183 & 0.796623 & -0.610556 \\
556.664 & 1557.68 & $-4.76043^{\prime} 10^{-6}$ & 0.0868007 & 0.550285 \\
823.33 & 956.422 & $-3.89351^{\prime} 10^{-6}$ & 0.0199445 & -1.45293 \\
224.057 & 999.806 & $-9.32687^{\prime} 10^{-7}$ & 0.027661 & -1.39276 \\
779.859 & 854.715 & $-7.88399^{\prime} 10^{-7}$ & 0.00765714 & -1.79925 \\
752.007 & 1123.49 & $1.8474513392943171^{\prime} 10^{-6}$ & 0.0059748 & -0.771908 \\
369.02 & 616.027 & -0.0000255421 & 0.00800571 & -0.698433 \\
249.933 & 1000. & $-1.0^{\prime} 10^{-6}$ & 0.00820914 & -1.12523 \\
1731.59 & 2371.61 & $-7.822^{\prime} 10^{-7}$ & 0.00431135 & -1.29539 \\
602.702 & 1213.28 & $-3.6232857813104305^{\prime} 10^{-6}$ & 0.00355905 & 0.832936 \\
196.354 & 1000. & $-1.0^{\prime} 10^{-6}$ & 0.00327965 & -0.852475 \\
103.866 & 900.002 & $-1.0^{\prime} 10^{-6}$ & 0.0056816 & -0.543118
\end{tabular}

\begin{tabular}{llllll}
$S$ & $T$ & $M$ & \multicolumn{1}{c}{ LL } & \multicolumn{1}{c}{ W } \\
$\mathrm{S}$ & 15 & 51 & 1 & 1375.9 & 0.0209988 \\
$\mathrm{~S}$ & 15 & 51 & 2 & 371.522 & 0.00624268 \\
$\mathrm{~S}$ & 15 & 51 & 3 & 350.805 & 0.0879385 \\
$\mathrm{~S}$ & 15 & 51 & 4 & 162.167 & 0.0238253 \\
$\mathrm{~S}$ & 15 & 51 & 5 & 100.316 & 0.0589567 \\
$\mathrm{~S}$ & 15 & 51 & 6 & 103.974 & 0.0714515 \\
$\mathrm{~S}$ & 15 & 51 & 7 & 263.167 & 0.121926 \\
$\mathrm{~S}$ & 15 & 51 & 8 & 97.2647 & 0.107716 \\
$\mathrm{~S}$ & 15 & 51 & 9 & 146.654 & 0.0388394 \\
$\mathrm{~S}$ & 15 & 51 & 10 & 361.641 & 0.194502 \\
$\mathrm{~S}$ & 15 & 51 & 11 & 98.9795 & 0.0195123 \\
$\mathrm{~S}$ & 15 & 51 & 12 & 99.5964 & 0.0323405
\end{tabular}
$\mathrm{s} \quad \mathrm{t} \quad \mathrm{a}$ $258.723 \quad 1120.46 \quad 0.0000544513$ $554.094 \quad 1555.41-4.80885^{\prime} 10^{-6}$ $200.9121000 . \quad-9.99868^{\prime} 10^{-7}$ $839.324961 .051-3.94057^{\prime} 10^{-6}$ $835.259 \quad 856.425 \quad-3.6369135573276787^{\prime} 10^{-7} \quad 0.00991082 \quad-2.25383$ $\begin{array}{llllll}703.926 & 1116.05 & 2.26447^{\prime} 10^{-6} & 0.009275 & -0.596541\end{array}$ 254.7231000 . $\quad-1.0008360002881072^{\prime} 10^{-6} \quad 0.0182986 \quad-1.26132$ $263.2881000 . \quad-9.37451^{\prime} 10^{-7} \quad 0.00609232-0.947037$ $\begin{array}{lllll}366.019 & 613.894 & -0.0000259335 & 0.00821953 & -0.816045\end{array}$ $\begin{array}{lllll}89.1085 & 899.826 & -9.76042^{\prime} 10^{-7} & 0.0111515 & -0.441494\end{array}$ $1606.63 \quad 2151.19-9.02219^{\prime} 10^{-7} \quad 0.00385305 \quad 2.74685$ $\begin{array}{lllllll}596.393 & 1207.45 & -3.6211473302594537^{\prime} & 10^{-6} & 0.0035101 & 0.855336\end{array}$

\section{Sample 15 Scan 52:}

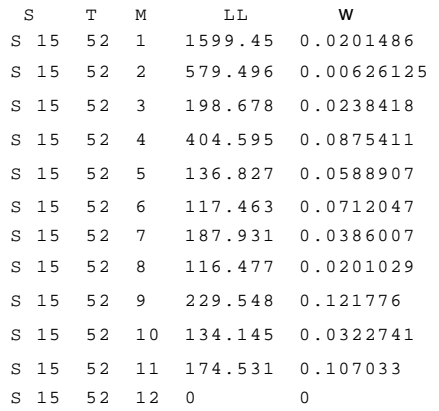

\begin{tabular}{|c|c|c|c|c|}
\hline s & $t$ & a & $\frac{\circ}{0}$ erg & $f$ \\
\hline 56.678 & 1133.08 & 0.0000541118 & 0.797993 & -0.636206 \\
\hline 6.38 & 1562.48 & $-4.80666^{\prime} 10^{-6}$ & 0.0882255 & 0.524488 \\
\hline 5.18 & 969.642 & $-3.91846^{\prime} 10^{-6}$ & 0.0201685 & -1.74685 \\
\hline 6.08 & 1000 & $-9.97734^{\prime} 10^{-7}$ & 0.0286584 & -1.30912 \\
\hline 8.004 & 854.612 & $-3.8085118239468053^{\prime} 10^{-7}$ & 0.00806863 & -1.84235 \\
\hline 0.226 & 1139.36 & $2.2289609606015463^{\prime} 10^{-6}$ & 0.00609844 & -1.92361 \\
\hline 6.636 & 616.095 & -0.000025701 & 0.00838284 & -0.700426 \\
\hline 78.86 & 2619.63 & $-8.28061^{\prime} 10^{-7}$ & 0.00445795 & 0.014603 \\
\hline 9.411 & 1000.01 & $-1 \cdot 10^{\prime}-6$ & 0.00728161 & -0.997919 \\
\hline 2.637 & 1211.73 & $-3.6235948048502768^{\prime} 10^{-6}$ & 0.00361569 & 0.905958 \\
\hline .82 & 1000 . & $-1.014811^{\prime} 10^{-6}$ & 0.00312501 & -0.6958 \\
\hline & 0 & 0 & 0 & 0 \\
\hline
\end{tabular}




\title{
DISTRIBUTION
}

\author{
BF Goodrich Aerospace \\ Université Laval \\ M. S. Miller \\ Prof. X. Maldague \\ Daimler Chrysler Corporation \\ U.S. Department of Energy \\ M. G. Forrest \\ R. P. Lindsey \\ Ford Motor Company \\ UT-Battelle, LLC \\ C. C. Gearhart \\ G. O. Allgood \\ R. Jahn \\ C. L. Carnal \\ B. Damiano \\ Hong Kong Polytechnic University \\ S. A. David \\ Wei Jin \\ L. B. Dunlap \\ J. E. Hardy \\ Industrial Materials Institute \\ S. W. Kercel (6) \\ Daniel Lévesque \\ R. A. Kisner (6) \\ Jean-Pierre Monchalin \\ W. W. Manges \\ Andre Moreau \\ T. J. McIntyre \\ W. A. Simpson \\ Lasson Technologies \\ R. W. Tucker \\ M. B. Klein (6) \\ G. S. Vanderhoofven \\ B. Pouet \\ National Science Foundation \\ F. Darema \\ D. G. Gorman \\ Virginia Polytechnic Institute and State \\ University \\ R. G. May \\ W. Ng \\ H. F. VanLandingham \\ SENSORS Magazine \\ T. Kevan \\ A. Wang \\ H. Xiao \\ $\underline{\text { Swiss Federal Institute of Technology- }}$ \\ Lausanne \\ P. Robert \\ TECNAR Automation LTÉE \\ M. Choquet \\ The Timken Company \\ G. V. Jeskey \\ R. V. Kolarik
}

